\title{
SINGLE CATALYST PARTICLE DIAGNOSTICS
}

USING MAGNETIC AND ELECTRIC FIELDS

Miguel Solsona 



\section{SINGLE CATALYST PARTICLE DIAGNOSTICS}

USING MAGNETIC AND ELECTRIC FIELDS

\section{DISSERTATION}

to obtain

the degree of doctor at the University of Twente, on the authority of rector magnificus, prof. dr. T.T.M. Palstra, on account of the decision of the Doctorate Board, to be publicly defended on Friday 26 ${ }^{\text {th }}$ of April 2019 at 14:45 by

Miguel Solsona, born on 17th of February 1986, in Barcelona, Catalonia, Spain. 
This dissertation is approved by:

Prof. dr. ir. A. van den Berg

Prof. dr. ir. B. M. Weckhuysen

Dr. W. Olthuis

Title: Single Catalyst Particle Diagnostics, using Magnetic and Electric Fields. Author: Miguel Solsona

Cover design: Binomic

Printed by: Gildeprint

ISBN: 978-90-365-4760-4

DOI: $10.3990 / 1.9789036547604$

URL: $\underline{\text { https://doi.org/10.3990/1.9789036547604 }}$

(C) 2019 Enschede, The Netherlands. All rights reserved. No parts of this thesis may be reproduced, stored in a retrieval system or transmitted in any form or by any means without permission of the author. Alle rechten voorbehouden. Niets uit deze uitgave mag worden vermenigvuldigd, in enige vorm of op enige wijze, zonder voorafgaande schriftelijke toestemming van de auteur. 
Committee members:

\section{Chairman:}

Prof. J. N. Kok

University of Twente

\section{Promotors:}

Prof. dr. ir. A. van den Berg

University of Twente

Prof. dr. ir. B. M. Weckhuysen

Utrecht University

Dr. W. Olthuis

University of Twente

\section{Members:}

Prof. N. Pamme

Prof. J. M. J. den Toonder

University of Hull

Prof. dr. H. Gardeniers

Eindhoven University of Technology

Prof. dr. ir. L. Abelmann

University of Twente

University of Twente

The research presented in this thesis has been carried out at BIOS lab on a chip group at the MESA+ Institute for Nanotechnology, University of Twente, the Netherlands, and the Inorganic Chemistry and Catalysis group, Debye Institute for Nanomaterials Science, Utrecht University, the Netherlands. This research was financially supported by the Netherlands Centre for Multiscale Catalytic Energy Conversion (MCEC), an NWO Gravitation program funded by the Ministry of Education, Culture and Science of the government of the Netherlands. 



\section{Table of Contents}

\section{Chapter 1}

Introduction.

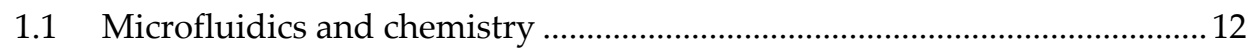

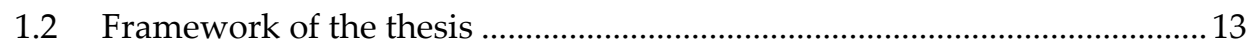

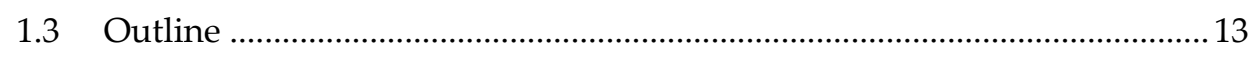

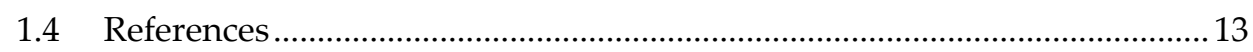

\section{Chapter 2}

Microfluidics and Catalyst Particles..........................................................15

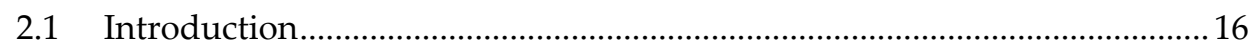

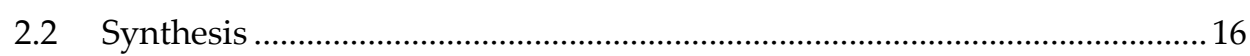

2.2.1 Metal nanoparticles........................................................................ 17

Homogeneous synthesis ................................................... 18

Droplet-based synthesis .........................................................21

Bimetallic NPs, quantum dots, silica and zeolites

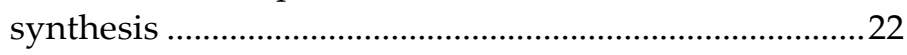

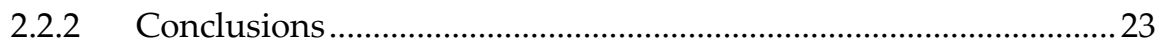

2.2.3 Future perspectives on particle synthesis .......................................23

Particle size and structure...................................................23

High throughput synthesis.................................................25

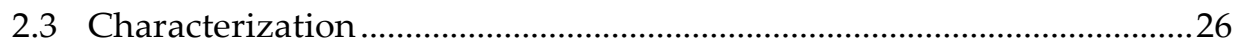

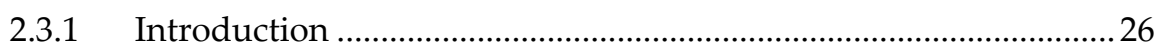

2.3.2 Characterization techniques .........................................................

Gas chromatography and Mass Spectrometry …………...26

Nuclear Magnetic Resonance ...............................................2 
Ultra-Violet visible Spectroscopy and X-ray

Absorption Spectroscopy …............................................... 27

Infrared and Raman Spectroscopy .................................. 28

2.3.3 In-situ characterization of nanoparticles in microfluidic

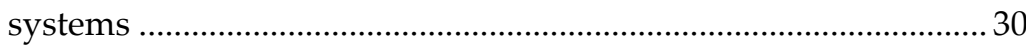

2.3.4 Activity of supported catalyst nanoparticles in microreactors ..... 33

Accessibility of active sites and deactivation studies 33

High throughput characterization..................................... 34

2.3.5 Characterization methods overview ............................................... 34

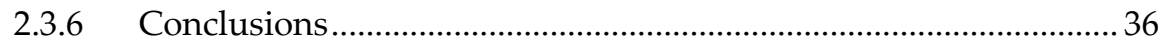

2.3.7 Perspective on catalyst characterization in microfluidics..............37

Single particle analysis ..................................................... 37

Single particle sorting ......................................................... 38

Particle storage and tagging ............................................. 38

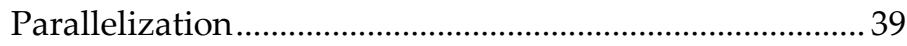

2.4 Perspective for fundamental understanding .........................................39

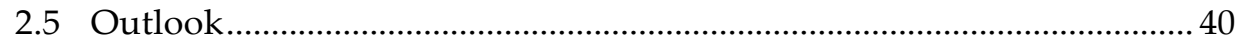

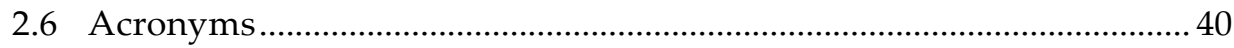

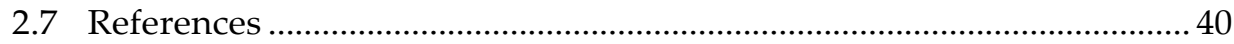

\section{Chapter 3}

Magnetophoretic Sorting of Single Catalyst Particles ................................55

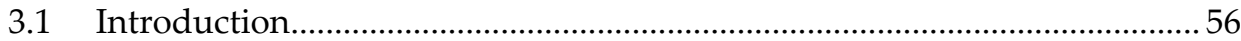

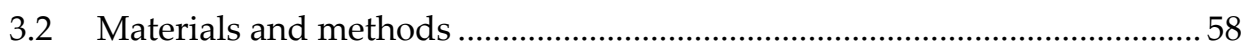

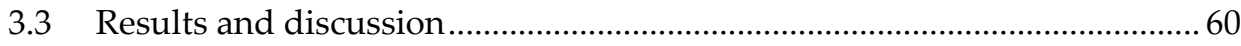

3.3.1 Magnetic properties and composition of sorted FCC particles .... 60

3.3.2 Acidity and accessibility of sorted FCC particles ...........................63

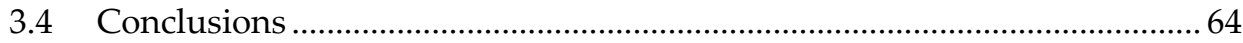

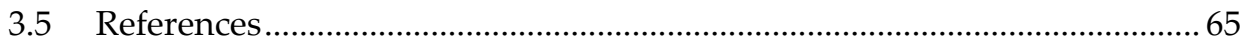




\section{Chapter 4}

First Steps Towards a Particle Sorting System using the Magnus Force.69

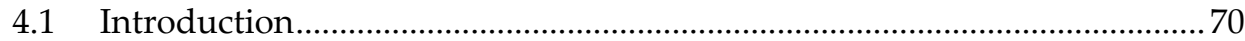

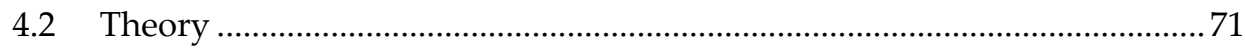

4.3 Materials and methods .............................................................................

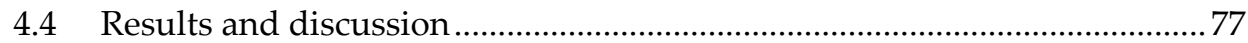

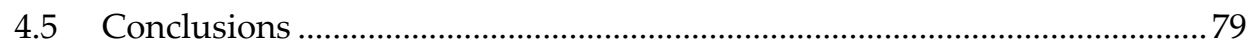

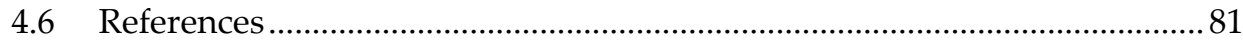

\section{Chapter 5}

Analysis of Catalyst Particle Activity by means of Impedance

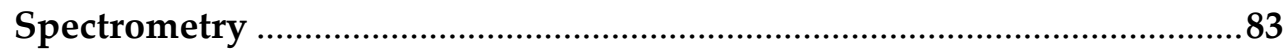

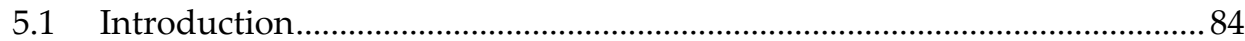

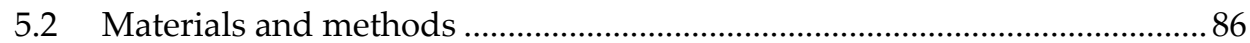

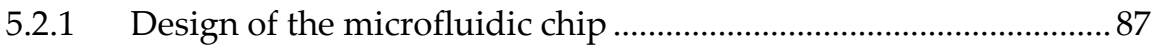

5.2.2 Measurement set-up working principle........................................... 87

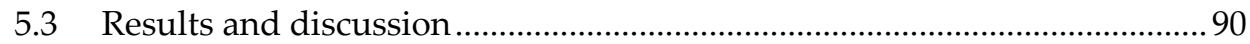

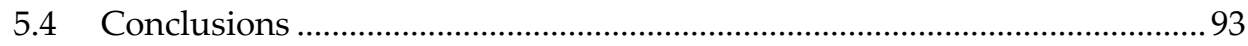

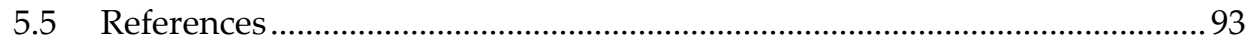

\section{Chapter 6}

Gradient in Electric Field for Particle Position Detection in Microfluidic

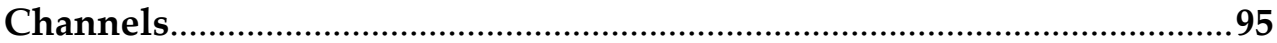

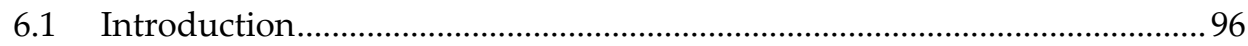

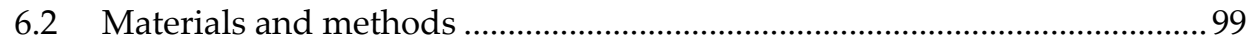

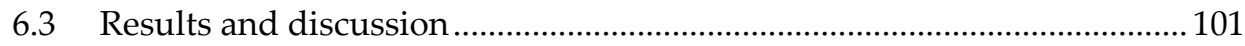

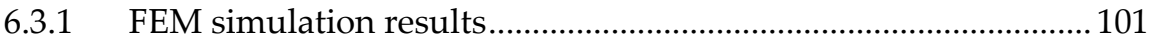

6.3.2 Experimental validation .................................................................. 103

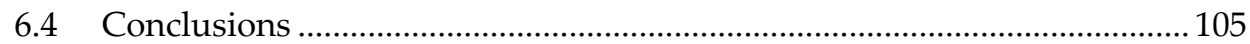

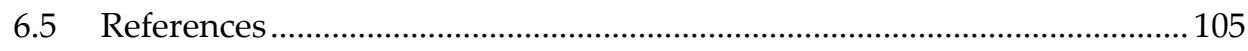




\section{Chapter 7}

Ion concentration Polarization for Microparticle Mesoporosity Differentiation 109

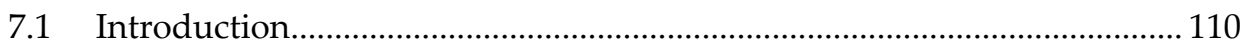

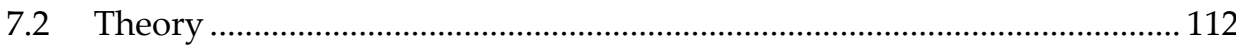

7.2.1 Nernst-Planck ............................................................................. 112

7.2.2 Transport number ......................................................................... 114

7.2.3 Current density ............................................................................... 116

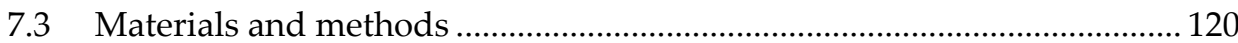

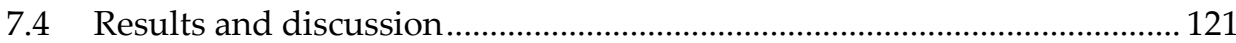

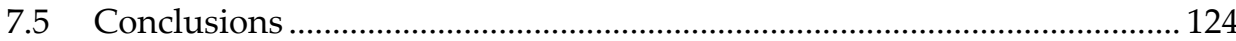

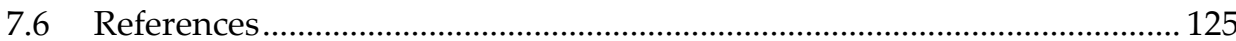

\section{Chapter 8}

\section{Supplementary results}

Single Catalyst Particle Reactor ………………….................................129

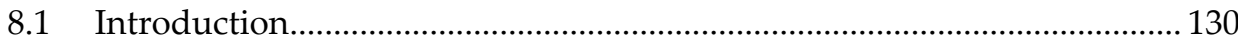

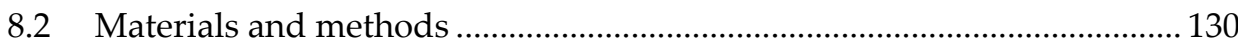

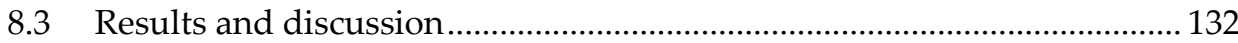

8.3.1 Analysis of carbon formation and removal from a single FCC particle .......................................................................................... 132

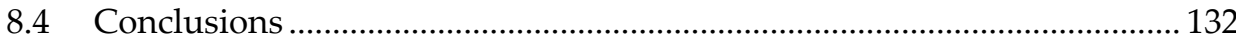

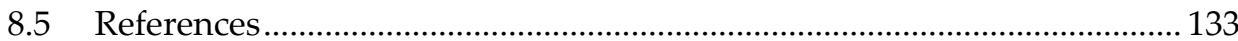

\section{Chapter 9}

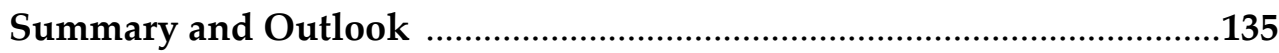

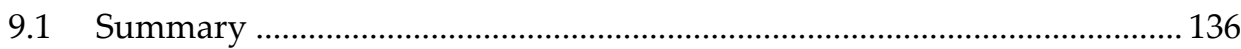

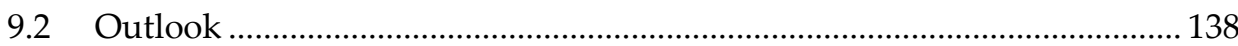




\section{Appendix}

A Magnetophoretic Sorting of Single Catalyst Particles (Chapter 3) ....142

A.1 Background on the theory of superparamagnetism .................................. 142

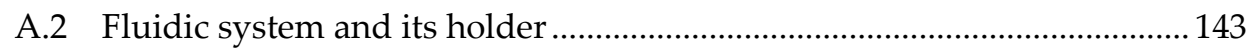

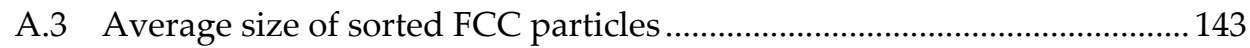

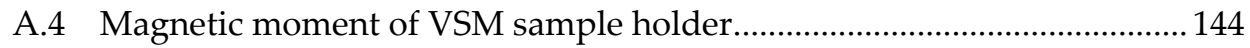

A.5 Micro-XRF average intensities per fraction............................................... 144

A.6 SEM-EDX results of black and orange spots on FCC particles ................. 145

A.7 EPR spectra and UV-vis diffuse reflectance spectra on FCC particles..... 146

A.8 Saturation magnetization of sorted FCC particles ..................................... 147

A.9 Magnetic moment of sorted FCC particles.................................................. 148

A.10 Fluorescence microscopy of sorted FCC particles...................................... 149

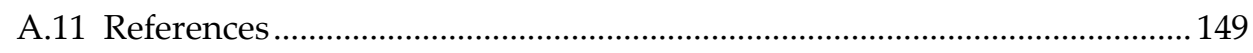

B First Steps Towards a Particle Sorting System using the Magnus Force

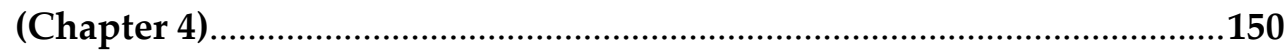

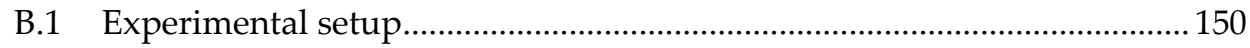

B.2 Magnetic moment of the janus particles.................................................. 151

B.3 Zig-zag particles inlet of the fluidic system ............................................. 151

C Analysis of Catalyst Particle Activity by means of Impedance Spectrometry (Chapter 5) .......................................................................152

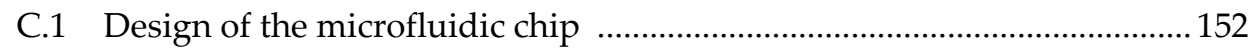

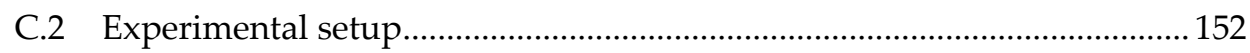

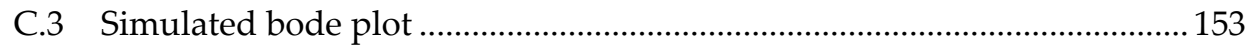

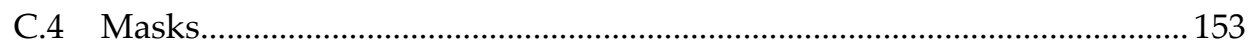

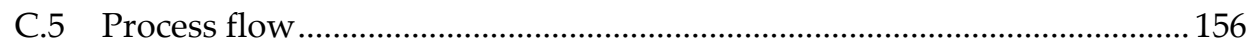

D Gradient in Electric Field for Particle Position Detection in Microfluidic Channels (Chapter 6) ..........................................................160

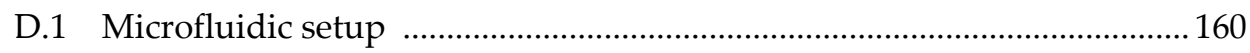

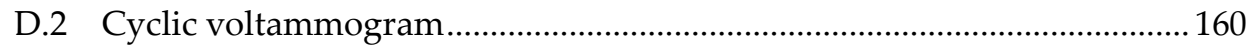

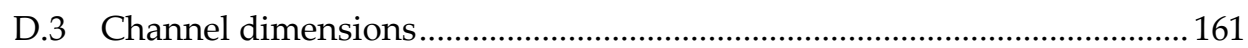

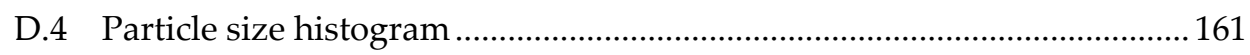




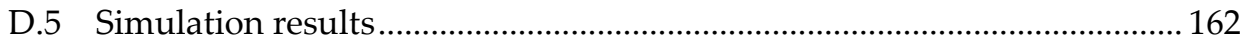

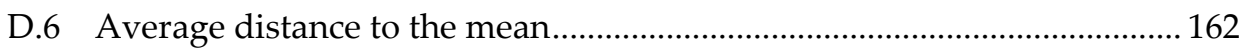

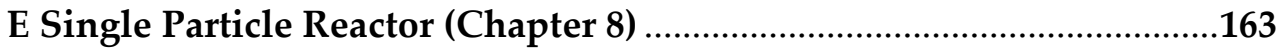

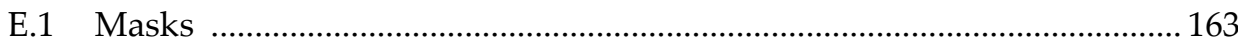

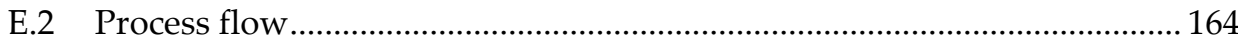

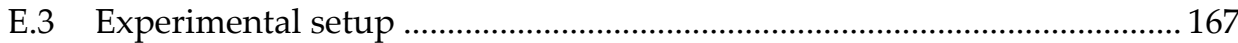

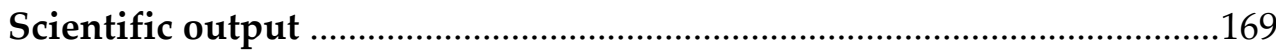

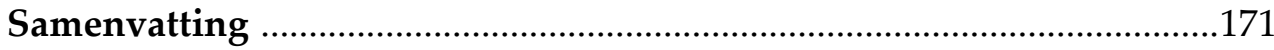

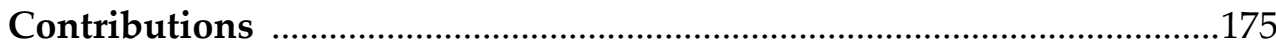

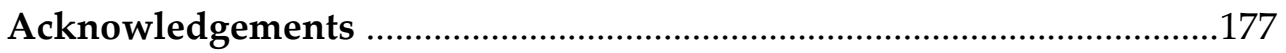




\section{Chapter 1}

\section{Introduction}

Tn this chapter the main topic of the thesis as well as its aim and framework are introduced. single particle level with respect to their activity. This research is focused on understanding the intra and inter-particle heterogeneity of heterogeneous catalyst particles. 


\subsection{Microfluidics and chemistry}

During the last couple of decades biological analysis and chemical synthesis have greatly benefited from microfluidic technologies. The small volumes and laminar flows that microfluidics enables, allow a better control of some of the parameters that affect the outcome of the system. ${ }^{1}$ This provided new capabilities to these fields that contributed to understanding of the system at the micro-scale. ${ }^{2}$ While microfluidics has been extensively used in biology, e.g. single cell analysis, ${ }^{3,4}$ in chemistry it is still far from its full potential.

Scaling down the reactor dimensions has well-known advantages in chemistry applications such as: using small reagents volumes, better selectivity control, less residence time, less energy consumption, better control of fast reactions and safer conditions. However, all these benefits come at a price, which is the low throughput and sometimes more complicated manoeuvrability. Nonetheless, microfluidics has attracted much attention from chemists, and during the last decade, has been extensively used to synthesize materials and analyse chemical reactions..$^{5-7}$ The use of milli and microfluidics for chemistry purposes, the so-called flow-chemistry, first started as a combination of organic chemistry and microfluidic reactors. ${ }^{8}$ Thereafter, the same technology was used to synthesise inorganic materials for different applications. Regarding catalysis, microfluidics has been widely used to synthesise and characterize heterogeneous catalysts. Furthermore, the extensive use of microfluidic technologies for biology purposes provided new technologies to separate and analyse microstructures such as cells. These technologies could be used for analysis of catalysts at the micro and nanoscale.

Over that past decade, microfluidic technologies have evolved in order to sort and characterize cells first with electric, and then with magnetic and acoustic fields. ${ }^{9}$ Regarding magnetic sorting, magnetophoresis, many examples in literature show the simplicity and yet efficacy of these systems when sorting cells. ${ }^{10,11}$ Normally, cells are labelled with superparamagnetic nanoparticles and therefore pulled by the magnetic material. ${ }^{12}$ In spite of their simplicity, these systems require good fabrication and process control. This is caused by the rapid decreasing magnetic field over distance of a typical magnetic material. ${ }^{11}$

There are many examples that use electric fields to analyse cells and particles in microfluidic systems. ${ }^{3,4}$ Flow impedance cytometry measures the dielectric properties of cells to detect differences in size and composition at single cell level and high throughput. This technology was developed due to the heterogeneity of cells in biological systems which provided ensemble averages. ${ }^{13}$ When looking at catalyst systems, we find a very similar trend. Heterogeneous catalyst supports have a similar size as cells and, due to their fabrication process, they are very intra and inter-particle heterogeneous. 
In this thesis we explore microfluidic technologies and try to improve and adapt them, in order to sort and analyse heterogeneous catalyst particles on activity.

\subsection{Framework of the thesis}

The aim of this thesis is to characterize and analyse catalyst particles using micro-technologies.

During the realization of this thesis I have been involved in MCEC (Multiscale Catalytic Energy Conversion), which is a multidisciplinary consortium of 6 research groups within The Netherlands, aiming at crossdisciplinary and multi-collaborative research. This consortium supplied the funding of all the research done in this thesis.

\subsection{Outline}

In this thesis different methods are reported that use magnetic and electric fields to analyse or sort catalytic microparticles at single particle level using microsystems. Chapter 2 reviews the utilization of microfluidic systems to synthesise and characterize catalyst particles, and, based on the past biological applications, tries to foresee the future steps on the combination of microfluidics and catalysis. Chapter 3 presents a new approach to sort catalyst particles by their magnetic susceptibility. Based on the previous chapter, Chapter 4 introduces the first steps to sort catalysts based on their magnetic material distribution by means of the Magnus force. Chapter 5 shows a microfluidic chip able to measure differences in metal content of catalytic particles by means of impedance spectroscopy. Chapter 6 uses impedance spectroscopy and a novel technology based on a gradient in electric field to detect the position of microparticles in microchannels. In Chapter 7 ion concentration polarization is used to measure differences in mesoporosity between polystyrene microparticles. Chapter 8 shows the first steps towards building a single catalytic microparticle reactor. Last but not least, Chapter 9 presents a summary and recommendations for future work on these topics. The appendices of chapters $3,4,5,6$ and 8 are shown in the last pages of this thesis.

\subsection{References}

1. Whitesides, G. M. The origins and the future of microfluidics. Nature 442, 368-373 (2006).

2. Jensen, K. F. Microchemical systems: Status, challenges, and opportunities. AIChE J. 45, 2051-2054 (1999).

3. Sun, T. \& Morgan, H. Single-cell microfluidic Impedance cytometry: A review. Microfluid. Nanofluidics 8, 423-443 (2010).

4. Yin, H. \& Marshall, D. Microfluidics for single cell analysis. Curr. Opin. Biotechnol. 23, 110-119 (2012). 
5. Elvira, K. S., I Solvas, X. C., Wootton, R. C. R. \& Demello, A. J. The past, present and potential for microfluidic reactor technology in chemical synthesis. Nat. Chem. 5, 905-915 (2013).

6. Hartman, R. L., McMullen, J. P. \& Jensen, K. F. Deciding whether to go with the flow: Evaluating the merits of flow reactors for synthesis. Angew. Chemie - Int. Ed. 50, 7502-7519 (2011).

7. Rodrigues, T., Schneider, P. \& Schneider, G. Accessing new chemical entities through microfluidic systems. Angew. Chemie - Int. Ed. 53, 57505758 (2014).

8. Jensen, K. F. Microfluidics for Chemical Synthesis: Flow Chemistry. arXiv Fluid Dyn. (2015).

9. Vilkner, T., Janasek, D. \& Manz, A. Micro total analysis systems. Recent developments. Anal. Chem. 76, 3373-3386 (2004).

10. Zborowski, M. \& Chalmers, J. J. Magnetophoresis: Fundamentals and Applications. in Wiley Encyclopedia of Electrical and Electronics Engineering (2015).

11. Pamme, N. Magnetism and microfluidics. Lab Chip 6, 24-38 (2006).

12. Pamme, N. \& Wilhelm, C. Continuous sorting of magnetic cells via onchip free-flow magnetophoresis. Lab Chip 6, 974-980 (2006).

13. Svahn, H. A. \& Van Den Berg, A. Single cells or large populations? Lab Chip 7, 544-546 (2007). 


\section{Chapter

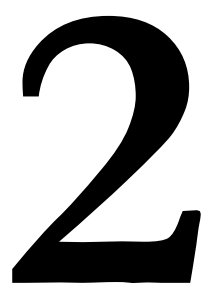

\section{Microfluidics and Catalyst Particles}

T $\mathrm{n}$ this chapter we discuss the latest advances and future perspective of 1 microfluidics for micro/nanoscale catalyst particle synthesis and analysis. In the first part we present an overview of the different methods to synthesize catalysts making use of microfluidics while the second part critically reviews catalyst particle characterization using microfluidics. The strengths and challenges of these approaches are highlighted with various showcases selected from recent literature. After each part, we give our opinion on the future perspectives of the combination of catalytic nanostructures and microfluidics. We anticipate that the synthesis and analysis of individual catalyst particles, creation of higher throughput by numbering up of microfluidic devices and better understanding of transport inside individual porous catalyst particles are the most important benefits of microfluidics for catalyst research.

This chapter is based on:

Solsona M.*, Vollenbroek J.C.*, Tregouet C.B.M.*, Nieuwelink A.E., Olthuis W., van den Berg .A., Weckhuysen B.M., and Odijk M., Microfluidics and Catalyst Particles, submitted.

* Authors contributed equally to this work 


\subsection{Introduction}

Catalysts are used in many different applications, such as fuel cells, exhaust gas catalytic conversion, water purification and chemicals production amongst others. In all these fields, the physical and chemical properties of the nanostructure of solid catalysts are of great importance. Over $80 \%$ of chemicals see a solid catalyst during their production, thus the role of nano-catalysts has become crucial in order to achieve a more sustainable society. ${ }^{1}$ The activity of these solid catalysts relies on their size, shape and accessibility of active sites. Therefore, more mono-disperse and uniform catalyst materials can tremendously increase their efficiency.

During the past two decades, microfluidics has been widely used to analyse and sort micro- and nano-structures, such as cells and microparticles, ${ }^{2,3}$ as well as to produce catalyst nanoparticles (NP) with a better control of their morphology and size. ${ }^{4-7}$ The small volumes, high operation speeds and small length scales in microfluidic devices, give more accurate control of the synthesis parameters affecting the overall quality of the catalyst materials prepared. Although microfluidics is a powerful tool for chemical analysis, ${ }^{8}$ its use in catalyst characterization is far from its full potential. As previously done in the cell biology field ${ }^{9}$ microfluidics could be an essential tool to characterize single catalyst particles at high throughput. Some critical reviews have focused on the synthesis of nanostructures using microfluidics, either as a general approach ${ }^{2,4}$ or focused on the microfluidic principle used. ${ }^{3}$

In this chapter, we first focus on the latest advances of the microfluidic synthesis of metal and metal oxide nanocatalysts. Second, we show how microfluidics has been used for in-situ characterization of nanocatalyst particles in terms of shape, size, activity, selectivity, and composition. Several characterization techniques working in synergy with microfluidics are discussed. After each section we introduce the possible future applications that microfluidics can open to the heterogeneous catalysis field. This review is intended to show an overview on synthesis and characterization, while we will highlight future opportunities enabled by the combination of both fields.

\subsection{Synthesis}

This section is focussed on the most recent approaches within the last 8 years. For a more extensive overview of the synthesis of nanostructures using microfluidics we refer the reader to other review articles. ${ }^{1-4}$ 
(a) Microfluidic channel

Homogeneous-synthesis approach

(b)

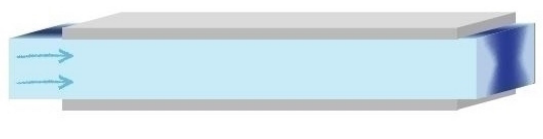

(c) Capillary-inside-capillary

Capillary

Larger capillary $\nearrow$
Droplet-synthesis approach

(d)

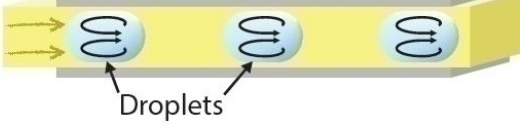

(e) Segmented flow

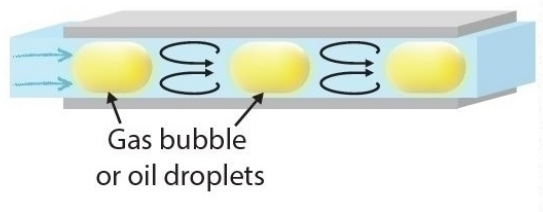

Figure 2.1. (a) Schematic drawing of a typical microfluidic chip used to synthesise NPs that consists of 3 inlets and 1 main channel. (b) Main channel section where the 3 different inlets merge into a single channel. (c) Tube inside tube configuration where the contact of both reagents occurs at the centre of the big channel. (d) Droplets of similar size formed by a typical microfluidic droplet generator and (e) mixing of the segmented flows separated by gas bubbles or oil droplets.

\subsubsection{Metal nanoparticles}

Metal nanoparticles exhibit very interesting catalytic, optical, chemical, electromagnetic and magnetic properties, all of them depending in a large degree on their size and composition. Normally, the NPs are produced in batch reactors with lack of controllability. Microfluidic NPs synthesis uses the same reactants as batch procedures, however, with a better control of the time and spatial distribution resulting in better size homogeneity. ${ }^{10-13}$ Also, due to the smaller dimensions, heat transfer, which is dominated by conduction and convection, can be supplied in a faster manner. ${ }^{14-17}$ Nevertheless, this comes at a price of low throughput and the development of more complex systems. Tables 2.1-3 show a summary of the structures, techniques, NPs size, temperature and reactants used to synthesize metal nanostructures, silica and zeolites using microfluidics. As can be seen, synthesis of metal NPs starts with a metal containing salt solution and a reducing agent, although sometimes the reducing agent is not needed.18,19 Thereafter, usually ligands or surfactants, the so-called capping agents, are used to control the shape and size of the structures. Time and contact between reagents is of great importance, both of them being 
better controlled by microfluidic systems. As stated in previous reviews,, 20 microfluidic synthesis uses two different techniques to contact the reagents, the homogeneous and the droplet-based approach.

\section{Homogeneous Synthesis}

Homogeneous synthesis consists of a simple mixing of reagents and surfactants using usually 2 or 3 inlets which combine in a single channel,11,21 altogether forming a $\mathrm{Y}$ or $\mathrm{T}$ shape depending on the angle of contact between the channels, see Figure 2.1a. The final shape of the NPs will depend on the contact and shear between the reagents, ${ }^{22}$ see Figure $2.1 \mathrm{~b}$. When reagents mix in a single channel, mixing occurs by diffusion. Due to the typical parabolic flow profile in microchannels, different velocities cause different residence times and consequently not homogeneous diffusion over the channel height, Figure 2.1b. Therefore, sometimes mixers are used to enhance the mixing and to provide a better control of the metal NPs size distribution and composition. These mixers use the inertia of the fluid to merge the reagents in a more vigorous manner. $21,23,24$ Typically, the synthesis is performed at room temperature, however higher temperatures are also used which can be very well controlled when integrated in the microfluidic chips. To control the contact between reagents, resulting in a better size distribution, flows can be divided in small sub-flows and then mixed together. ${ }^{25-27}$ Also, to avoid clogging of the channels due to particles agglomeration, a glass capillary injection into a bigger tube can be used.28,29 This technique consists of introducing a glass capillary inside a bigger channel in order to contact both reagents in the middle of the channel. By doing so, the nucleation of NPs is confined in the bigger channel's centre which avoids the particles getting stuck on the walls, as shown in Figure 2.1c. Table 2.1 presents an overview of the different studies found that used homogeneous flows to synthesise metal NPs.

Table 2.1. Some characteristics of synthesis of metal nano-structures using homogeneous flows. For a list of used acronyms, refer to the end of the chapter.

\begin{tabular}{|c|c|c|c|c|c|}
\hline Material & Micro-reactor & Size & Temp. & Reactants & Ref. \\
\hline Au NPs & Glass & $11.5 \mathrm{~nm}$ & NS & $\mathrm{HAuCl}_{4}+\mathrm{SC}+$ Tannic acid & 30 \\
\hline $\mathrm{Au}$ NPs & Silicon-glass & $1 \mathrm{~nm}$ & NS & $\mathrm{HAuCl}_{4}+\mathrm{NaBH}_{4}+\mathrm{PVP}$ & 31 \\
\hline $\mathrm{Au} \mathrm{NPs}$ & Glass Capillary & $48-135 \mathrm{~nm}$ & RT & $\mathrm{HAuCl}_{4}+\mathrm{PVP}+\mathrm{AA}$ & 32 \\
\hline Au NRs & PTFE & $\approx 50 \mathrm{~nm}$ & NS & $\begin{array}{l}\mathrm{HAuCl}_{4}+\mathrm{CTABr}+\text { Tannic acid + } \\
\mathrm{AgNO}_{3}+\mathrm{AA}+\mathrm{NaBH}_{4}+\mathrm{PEG}\end{array}$ & 33 \\
\hline $\mathrm{Au}$ NPs & PDMS & $\approx 40 \mathrm{~nm}$ & NS & Seeds $+\mathrm{HAuCl}_{4}+\mathrm{AA}$ & 34 \\
\hline $\mathrm{Au}$ NPs & Stainless steel & $24-36 \mathrm{~nm}$ & NS & $\mathrm{HAuCl}_{4}$ & 18 \\
\hline $\begin{array}{l}\text { Au } \\
\text { hollow } \\
\text { NPs }\end{array}$ & PTFE & $\approx 40 \mathrm{~nm}$ & RT & $\mathrm{HAuCl}_{4}+\mathrm{NaBH}_{4}+\mathrm{PVP}$ & 35 \\
\hline $\mathrm{Au} \mathrm{NPs}$ & PDMS & $\approx 125 \mathrm{~nm}$ & $\begin{array}{l}22-50 \\
{ }^{\circ} \mathrm{C}\end{array}$ & $\mathrm{HAuCl}_{4}+\mathrm{PDMS}$ curing agent & 19 \\
\hline $\mathrm{Au}$ NPs & PE & $2-37 \mathrm{~nm}$ & RT & $\mathrm{HAuCl}_{4}+\mathrm{SC}+\mathrm{NaBH}_{4}$ & 36 \\
\hline
\end{tabular}




\begin{tabular}{|c|c|c|c|c|c|}
\hline & & & & $\mathrm{HAuCl}_{4}+\mathrm{MHA}+\mathrm{NaBH}_{4}$ & 36 \\
\hline & & & & $\mathrm{HAuCl}_{4}+\mathrm{CTAB}+\mathrm{NaBH}_{4}+\mathrm{AA}$ & 36 \\
\hline & & & & $\begin{array}{l}\mathrm{HAuCl}_{4}+\mathrm{CTAB}+\mathrm{NaBH}_{4}+\mathrm{AA}+ \\
\mathrm{AgNO}_{3}\end{array}$ & 36 \\
\hline $\mathrm{Au}$ NPs & PTP & NS & NS & $\mathrm{HAuCl}_{4}+\mathrm{DMSA}+\mathrm{NaBH}_{4}$ & 37 \\
\hline Au NPs & $\begin{array}{l}\text { Teflon and } \\
\text { PDMS }\end{array}$ & $3-25 \mathrm{~nm}$ & RT & $\mathrm{HAuCl}_{4}+\mathrm{AA}+\mathrm{NaOH}$ & 23 \\
\hline Au NPs & PTE & $4.3-8.7 \mathrm{~nm}$ & $\begin{array}{l}25- \\
60^{\circ} \mathrm{C}\end{array}$ & $\begin{array}{l}\mathrm{HAuCl}_{4}+\text { Dodecanethol + } \mathrm{ET}_{3} \mathrm{SiH}+ \\
\text { THF }\end{array}$ & 38 \\
\hline $\mathrm{Au}$ NPs & PTE & $\approx 100 \mathrm{~nm}$ & NS & $\mathrm{HAuCl}_{4}+\mathrm{H} 2 \mathrm{SO} 4$ & 39 \\
\hline $\mathrm{Au}$ NPs & 3D-Printed & $\approx 10 \mathrm{~nm}$ & NS & $\mathrm{HAuCl}_{4}+\mathrm{SC}+\mathrm{NaBH}_{4}$ & 40 \\
\hline $\mathrm{Au}$ NPs & PDMS & $\approx 5 \mathrm{~nm}$ & $35^{\circ} \mathrm{C}$ & $\mathrm{HAuCl}_{4}+\mathrm{CTAB}+\mathrm{NaBH}_{4}$ & 40 \\
\hline $\mathrm{Au}$ NPs & PEEK & $1-2 \mathrm{~nm}$ & $100^{\circ} \mathrm{C}$ & $\mathrm{HAuCl}_{4}+\mathrm{SC}$ & 10 \\
\hline $\mathrm{Au}$ NPs & Glass capillary & $1.8 \mathrm{~nm}$ & $100^{\circ} \mathrm{C}$ & $\mathrm{HAuCl}_{4}+\mathrm{SC}$ & 11 \\
\hline Au NPs & $\begin{array}{l}\text { Stainless steel or } \\
\text { Teflon }\end{array}$ & $1.5-181 \mathrm{~nm}$ & RT & $\mathrm{HAuCl}_{4}+\mathrm{AA}$ or $\mathrm{NaBH}_{4}$ & 41 \\
\hline Au NPs & Teflon & $\approx 40 \mathrm{~nm}$ & RT & $\mathrm{HAuCl}_{4}+\mathrm{NaOH}+$ Glucose & 42 \\
\hline $\mathrm{Au}$ NPs & Silicon-Glass & $\approx 40 \mathrm{~nm}$ & RT & $\mathrm{HAuCl}_{4}+\mathrm{SC}$ & 43 \\
\hline $\mathrm{Au}$ NPs & PVDF & & & $\mathrm{HAuCl}_{4}+\mathrm{NaBH}_{4}$ & 24 \\
\hline $\mathrm{Au}$ NPs & $\begin{array}{l}\text { Low Temp. } \\
\text { Ceramic }\end{array}$ & $3 \mathrm{~nm}$ & & $\mathrm{HAuCl}_{4}+\mathrm{NaBH}_{4}+\mathrm{MUA}$ & 44 \\
\hline $\mathrm{Au}$ NRs & Rotating tube & $\approx 30 \mathrm{~nm}$ & RT & $\begin{array}{l}\mathrm{HAuCl}_{4}+\text { Acetylacetone }+\mathrm{CTAB}+ \\
\mathrm{AgNO}_{3}+\text { Carbonate buffer }\end{array}$ & 45 \\
\hline Ag NPs & PDMS & $5-12 \mathrm{~nm}$ & RT & $\mathrm{AgNO}_{3}+\mathrm{NaBH}_{4}+\mathrm{AA}+\mathrm{PVP}$ & 26 \\
\hline Ag NPs & PDMS & & RT & $\mathrm{AgNO}_{3}+\mathrm{OPD}$ & 22 \\
\hline Ag NPs & Glass capillary & $3.1-9.3 \mathrm{~nm}$ & $\mathrm{RT}$ & $\mathrm{AgNO}_{3}+\mathrm{SC}+\mathrm{NaBH}_{4}$ & 28 \\
\hline Ag NPs & Quartz spiral & $5-40 \mathrm{~nm}$ & $\begin{array}{l}130- \\
150^{\circ} \mathrm{C}\end{array}$ & $\mathrm{Ag}\left(\mathrm{NH}_{3}\right)_{2}+$ Glucose + PVP & 46 \\
\hline Ag NPs & ETFE and PTFE & $5.3-7 \mathrm{~nm}$ & $90^{\circ} \mathrm{C}$ & $\mathrm{AgNO}_{3}+\mathrm{NaOH}+$ C.Platiclady & 47 \\
\hline Pd NPs & Silicon-Glass & $1 \mathrm{~nm}$ & $\begin{array}{l}60 \& \& \\
280^{\circ} \mathrm{C}\end{array}$ & $\begin{array}{l}\text { Pd Acetate + Toluene + Methanol + } \\
\text { OLA + TOP }\end{array}$ & 48 \\
\hline $\begin{array}{l}\mathrm{Fe}_{3} \mathrm{O}_{4} \\
\mathrm{NPs}\end{array}$ & Stainless steel & $\approx 4 \mathrm{~nm}$ & $\begin{array}{l}180 \& \& \\
280^{\circ} \mathrm{C}\end{array}$ & $\begin{array}{l}\mathrm{Fe}(\text { acac })_{3}+\mathrm{TEG}+\mathrm{Ethanol}+\mathrm{EA}+ \\
\mathrm{HCl}\end{array}$ & 33 \\
\hline $\begin{array}{l}\text { FeZn } \\
\text { NPs }\end{array}$ & Stainless steel & $\approx 5 \mathrm{~nm}$ & $\begin{array}{l}30 \& \& \\
150^{\circ} \mathrm{C}\end{array}$ & $\mathrm{FeCl}_{2}+\mathrm{ZnCl}_{2}+\mathrm{NaBH}_{4}+\mathrm{PVP}$ & 49 \\
\hline $\begin{array}{l}\mathrm{Fe}_{3} \mathrm{O}_{4} \\
\mathrm{NPs}\end{array}$ & PTFE & $\approx 140 \mathrm{~nm}$ & $60^{\circ} \mathrm{C}$ & $\mathrm{FeCl}_{2}+\mathrm{FeCl}_{3}+\mathrm{NaBH}_{4}+\mathrm{PVP}$ & 12 \\
\hline $\begin{array}{l}\mathrm{Fe}_{3} \mathrm{O}_{4} \\
\mathrm{NPs}\end{array}$ & Hastelloy & $4.9 \mathrm{~nm}$ & $250^{\circ} \mathrm{C}$ & $\begin{array}{l}\mathrm{Fe}(\mathrm{acac})_{3}+\text { Anisole }+(\mathrm{HOOC}-\mathrm{PEG}- \\
\mathrm{COOH})+ \text { oleyamine }\end{array}$ & 50 \\
\hline $\mathrm{Cu}$ NPs & Stainless-Steel & $\approx 10 \mathrm{~nm}$ & RT & $\mathrm{CuSO}_{4}+\mathrm{NaBH}_{4}+\mathrm{PVP}$ & 25,26 \\
\hline $\mathrm{Cu}$ NPs & Teflon & $135.6 \mathrm{~nm}$ & RT & $\begin{array}{l}\mathrm{CuCl}_{2}+\mathrm{THF}+\mathrm{LiBEt} 3 \mathrm{H}+\mathrm{SB}_{12}+ \\
\text { Acetone + Ethanol }\end{array}$ & 51 \\
\hline $\begin{array}{l}\mathrm{CoFe}_{2} \mathrm{O}_{4} \\
\mathrm{NPS}\end{array}$ & PDMS and PTFE & $5-15 \mathrm{~nm}$ & $98^{\circ} \mathrm{C}$ & $\mathrm{CoCl}_{2}+\mathrm{FeCl}_{3}+\mathrm{TMAOH}$ & 29 \\
\hline Ni NPs & - & $10 \mathrm{~nm}$ & $220^{\circ} \mathrm{C}$ & $\begin{array}{l}\mathrm{Ni}(\text { acac })_{2}+\text { Oleylamine }+ \\
\text { Octadencene + Trioctylphosphine }\end{array}$ & 13 \\
\hline Ni NPs & Stainless steel & $5-9 \mathrm{~nm}$ & $80^{\circ} \mathrm{C}$ & $\mathrm{NiSO}_{4}+\mathrm{N}_{2} \mathrm{HH}_{4}+\mathrm{PVP}+\mathrm{NaOH}$ & 52 \\
\hline Ni NPs & Stainless-steel & $5.3-7.4 \mathrm{~nm}$ & $\begin{array}{l}60- \\
120^{\circ} \mathrm{C}\end{array}$ & $\begin{array}{l}\mathrm{NiCl}_{2}+\mathrm{Hydrazine} \text { monohydrate }+ \\
\mathrm{NaOH}+\mathrm{EG}\end{array}$ & 53 \\
\hline Pt NPs & PTFE & $2.8 \mathrm{~nm}$ & RT & $\begin{array}{l}\mathrm{H}_{2} \mathrm{PtCl}_{6}+\mathrm{PVP}+\mathrm{HMP}+\mathrm{UV} \\
(365 \mathrm{~nm})\end{array}$ & 54 \\
\hline Pt NPs & Copper & $5 \mathrm{~nm}$ & RT & $\mathrm{K}_{2} \mathrm{PtCl}_{6}+\mathrm{NaBH}_{4}+\mathrm{PVP}$ & 55 \\
\hline Pt NPs & Glass & $1.4 \mathrm{~nm}$ & $0^{\circ} \mathrm{C}$ & $\mathrm{H}_{2} \mathrm{PtCl}_{6} \mathrm{NaBH}_{4}+\mathrm{PVP}$ & 56 \\
\hline
\end{tabular}


Table 2.2. Some characteristics of synthesis of metal nano-structures using droplets. For a list of used acronyms, refer to the end of the chapter.

\begin{tabular}{|c|c|c|c|c|c|}
\hline Material & Micro-reactor & Size & Temp. & Reactants & Ref. \\
\hline Au NSs & PDMS & $20-50 \mathrm{~nm}$ & NS & $\begin{array}{l}\text { Seeds }+\mathrm{HCl}+\mathrm{AA}+\mathrm{AgNO}_{3}+\mathrm{PVP}+ \\
\mathrm{DMF}\end{array}$ & 57 \\
\hline Au NPs & PDMS & $\approx 4 \mathrm{~nm}$ & RT & $\begin{array}{l}\mathrm{HAuCl}_{4}+\mathrm{BMIM} \mathrm{Tf}_{2} \mathrm{~N}+ \\
\text { Methylimidazole + BMIM-BH}\end{array}$ & 58 \\
\hline Au NPs & PDMS & $\approx 4 \mathrm{~nm}$ & & 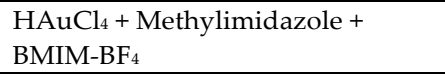 & 59 \\
\hline $\mathrm{Cu}$ NPs & PDMS & $\approx 10 \mathrm{~nm}$ & & $\begin{array}{l}\mathrm{CuSO}_{4}+\mathrm{NaBH}_{4}+\mathrm{PVP}+\mathrm{NH}_{3}+ \\
\mathrm{NaOH}\end{array}$ & 60 \\
\hline $\mathrm{Ag} \mathrm{NCs}$ & $\begin{array}{l}\text { PTFE and Glass } \\
\text { capillary }\end{array}$ & $30-100 \mathrm{~nm}$ & $150^{\circ} \mathrm{C}$ & $(\mathrm{Ag}$ Seeds $)+\mathrm{AgNO}_{3}+\mathrm{PVP}+\mathrm{EG}$ & 61 \\
\hline Au NPs & PTFE and PEEK & $2.5-4 \mathrm{~nm}$ & RT & $\begin{array}{l}\mathrm{HAuCl}_{4}+\text { Photoinitiator + AA + } \\
\mathrm{PVP}\end{array}$ & 62 \\
\hline Pd NPs & $\begin{array}{l}\text { PTFE and Glass } \\
\text { capillary }\end{array}$ & $8.1-9.1 \mathrm{~nm}$ & $80^{\circ} \mathrm{C}$ & $\mathrm{Na}_{2} \mathrm{PdCl}_{4}+\mathrm{PVP}+\mathrm{AA}+\mathrm{KBr}$ & 63 \\
\hline $\mathrm{Ag} \mathrm{NCs}$ & $\begin{array}{l}\text { Fused silica and } \\
\text { PTFE }\end{array}$ & $30-100 \mathrm{~nm}$ & $150^{\circ} \mathrm{C}$ & $(\mathrm{Ag}$ Seeds $)+\mathrm{AgNO}_{3}+\mathrm{PVP}+\mathrm{EG}$ & 61 \\
\hline $\begin{array}{l}\mathrm{Fe}_{3} \mathrm{O}_{4} \\
\mathrm{NPs}\end{array}$ & $\begin{array}{l}\text { PTFE and Glass } \\
\text { capillary }\end{array}$ & $3.6 \mathrm{~nm}$ & NS & $\mathrm{FeCl}_{2}+\mathrm{FeCl}_{3}+$ Dextran $+\mathrm{NH}_{3} \mathrm{OH}$ & 64 \\
\hline $\begin{array}{l}\text { FeMn } \\
\text { NPs }\end{array}$ & PDMS & $\approx 3.6 \mathrm{~nm}$ & NS & $\mathrm{FeSO}_{4}+\mathrm{MnCl}_{2}+\mathrm{E} . \mathrm{Coli}+\mathrm{PEG}-\mathrm{PFPE}$ & 65 \\
\hline Pd NPs & $\begin{array}{l}\text { PTFE and Glass } \\
\text { capillary }\end{array}$ & $9-37 \mathrm{~nm}$ & $80^{\circ} \mathrm{C}$ & $\mathrm{Na}_{2} \mathrm{PdCl}_{4}+\mathrm{PVP}+\mathrm{AA}+\mathrm{KBr}$ & 66 \\
\hline Pt NPs & PTFE & $15 \mathrm{~nm}$ & RT & $\begin{array}{l}\mathrm{H}_{2} \mathrm{PtCl}_{6}+\mathrm{PVP}+\mathrm{HMP}+\mathrm{UV} \\
(365 \mathrm{~nm})\end{array}$ & 67 \\
\hline Ag NPs & $\begin{array}{l}\text { PEEK and } \\
\text { PMMA }\end{array}$ & $5-20 \mathrm{~nm}$ & NS & $\mathrm{AgNO}_{3}+\mathrm{KOH}$ & 68 \\
\hline Au NPs & Silicon-Glass & $\approx 3-8 \mathrm{~nm}$ & $100^{\circ} \mathrm{C}$ & $\mathrm{HAuCl}_{4}+\mathrm{NaBH}_{4}$ & 69 \\
\hline
\end{tabular}

Table 2.3. Some characteristics of synthesis of bimetalic, quantum dots, silica and zeolite nanostructures using microfluidics. For a list of used acronyms, refer to the end of the chapter.

\begin{tabular}{|c|c|c|c|c|c|}
\hline Material & Micro-reactor & Size & Temp. & Reactants & Ref. \\
\hline $\mathrm{CoFe}_{2} \mathrm{O}_{4} \mathrm{NPs}$ & $\begin{array}{l}\text { PDMS and } \\
\text { PTFE }\end{array}$ & NS & $98^{\circ} \mathrm{C}$ & $\mathrm{CoCl}_{2}+\mathrm{FeCl}_{3}+\mathrm{TMAOH}$ & 29 \\
\hline AuPd NPs & $\begin{array}{l}\text { PTFE and } \\
\text { PEEK }\end{array}$ & $10 \mathrm{~nm}$ & NS & $\begin{array}{l}\text { Pd Seeds + KBr + PVP + AA + } \\
\mathrm{HAuCl}_{4}\end{array}$ & 66 \\
\hline AuPd NPs & Zirconia & $\begin{array}{l}0.9-2.8 \\
\mathrm{~nm}\end{array}$ & NS & $\begin{array}{l}\mathrm{HAuCl}_{4}+\mathrm{H}_{2} \mathrm{PdCl}_{4}+\mathrm{NaBH}_{4}+ \\
\mathrm{PVP}\end{array}$ & 27 \\
\hline PtBi NPs & Capillary & & $\begin{array}{l}260 \& \\
350^{\circ} \mathrm{C}\end{array}$ & $\begin{array}{l}\mathrm{BiNO}_{3}+\mathrm{H}_{2} \mathrm{PtCl}_{6}+\mathrm{NaBH}_{4}+ \\
\mathrm{PVP}+\mathrm{EG}+\mathrm{PG}\end{array}$ & 70 \\
\hline FePt NPs & Stainless steel & $\approx 2 \mathrm{~nm}$ & $120^{\circ} \mathrm{C}$ & $\begin{array}{l}\mathrm{FeCl}_{2}+\mathrm{H}_{2} \mathrm{PtCl}_{6}+\mathrm{SnCl}_{2}+\mathrm{PVP}+ \\
\mathrm{NaBH}_{4}\end{array}$ & 71 \\
\hline $\begin{array}{l}\mathrm{Ag} / \mathrm{CuO}_{2} \text { core- } \\
\text { shell NPs }\end{array}$ & PTFE & $\approx 100 \mathrm{~nm}$ & & $\begin{array}{l}(\mathrm{Ag} \text { Seeds })+\mathrm{CuSO}_{4}+\mathrm{NaOH}+ \\
\mathrm{AA}\end{array}$ & 72 \\
\hline CdSe NPs & & $5 \mathrm{~nm}$ & $250^{\circ} \mathrm{C}$ & $\mathrm{CdO}+\mathrm{Se}+\mathrm{TOP}+\mathrm{E}$ acid & 73 \\
\hline CdSe NPs & PMMA & $4 \mathrm{~nm}$ & $250^{\circ} \mathrm{C}$ & $\mathrm{CdO}+\mathrm{Se}+\mathrm{TOP}+$ Oleic acid & 74 \\
\hline CdSe NPs & Capillary & 3-10 nm & $250^{\circ} \mathrm{C}$ & $\begin{array}{l}\mathrm{Cd}(\mathrm{OAc})_{2}+\mathrm{Se}+\mathrm{TOP}+\text { Oleic } \\
\text { acid }\end{array}$ & 75 \\
\hline CdSe NPs & PTFE reactor & $\approx 3 \mathrm{~nm}$ & $300^{\circ} \mathrm{C}$ & $\mathrm{CdO}+\mathrm{Se}+\mathrm{TOP}+$ Oleic acid & 76 \\
\hline
\end{tabular}




\begin{tabular}{|c|c|c|c|c|c|}
\hline PbS NPs & $\begin{array}{l}\text { PTFE and } \\
\text { PEEK }\end{array}$ & $\approx 5 \mathrm{~nm}$ & $\begin{array}{l}80-150 \\
{ }^{\circ} \mathrm{C}\end{array}$ & $\mathrm{Pb}(\mathrm{OAc})_{2}+\mathrm{TMS}_{2} \mathrm{~S}+\mathrm{Se}$ & 77 \\
\hline $\mathrm{SiO}_{2}$ & PDMS & $\begin{array}{l}8 \mu \mathrm{m}, 3 \\
\mathrm{~nm} \text { pores }\end{array}$ & RT & $\begin{array}{l}\text { TEOS + CTAB + HCl + ABIL } \\
\text { EM } 90\end{array}$ & 78 \\
\hline $\mathrm{SiO}_{2}$ & PDMS & $10-30 \mu \mathrm{m}$ & NS & $\mathrm{TEOS}+\mathrm{P} 123+\mathrm{HNO}_{3}$ & 79 \\
\hline $\mathrm{SiO}_{2}$ & PDMS & $\begin{array}{l}800 \mathrm{~nm} \\
\text { hollow }\end{array}$ & RT & $\mathrm{TEOS}+\mathrm{NH}_{4} \mathrm{OH}+\mathrm{CTAB}$ & 80 \\
\hline $\mathrm{SiO}_{2}$ & PDMS & $34 \mu \mathrm{m}$ & RT & TEOS + P104 + Ethanol + HCl & 81 \\
\hline Zeolite A & $\begin{array}{l}\text { Stainless steel } \\
\text { and PTFE }\end{array}$ & $\begin{array}{l}0.9-1.5 \\
\mu \mathrm{m}\end{array}$ & $90^{\circ} \mathrm{C}$ & $\mathrm{NaOH}+\mathrm{SA}+\mathrm{SC}$ & 82 \\
\hline Zeolite A & $\begin{array}{l}\text { Stainless steel } \\
\text { and PTFE }\end{array}$ & $\approx 400 \mathrm{~nm}$ & $90^{\circ} \mathrm{C}$ & $\mathrm{NaOH}+\mathrm{SA}+\mathrm{SC}$ & 83 \\
\hline Zeolite A & $\begin{array}{l}\text { Stainless steel } \\
\text { and PTFE }\end{array}$ & $\begin{array}{l}70-1500 \\
\mathrm{~nm}\end{array}$ & $\begin{array}{l}80-100 \\
{ }^{\circ} \mathrm{C} \\
\end{array}$ & $\mathrm{NaOH}+\mathrm{SA}+\mathrm{SC}$ & 84 \\
\hline Zeolite A & PFPE and PFA & $\approx 100 \mathrm{~nm}$ & $100^{\circ} \mathrm{C}$ & $\mathrm{NaOH}+\mathrm{TMAOH}+\mathrm{TEOS}$ & 85 \\
\hline
\end{tabular}

\section{Droplet-based Synthesis}

Metal NPs synthesis in droplets is based on the enhanced mixing created by the droplets or segmented flows. Normally, reagents contact each other in aqueous solutions and upon mixing with an organic phase either small aqueous or organic droplets are formed depending on the wetting properties of the chip, see Figure 2.1d. Droplet-based synthesis offers a better mixing of the regents due to the small volume of the droplets (nano-liter to pico-liter regime), which is a great advantage for the particle size distribution and composition. It was demonstrated that the mixing of reagents in droplets is very sensitive to the initial formation of the droplet and that the time of mixing can be reduced down to few ms. ${ }^{86}$ Although mixing in droplets occurs spontaneously via recirculation of the liquid inside, ${ }^{87}$ sometimes pinched, serpentine or spiral zones are introduced in order to enhance mixing. ${ }^{61,63}$ Frequently, droplets are formed by oil or gas bubbles and the space between droplets, the so-called continuous phase, forms a segmented flow which is used to mix the reagents, see Figure 2.1e. This approach is found to better merge both reagents where the mixing is enhanced by the slip velocity of both phases. ${ }^{69}$ Table 2.2 shows an overview of the different studies found that used droplets to synthesise metal NPs. 

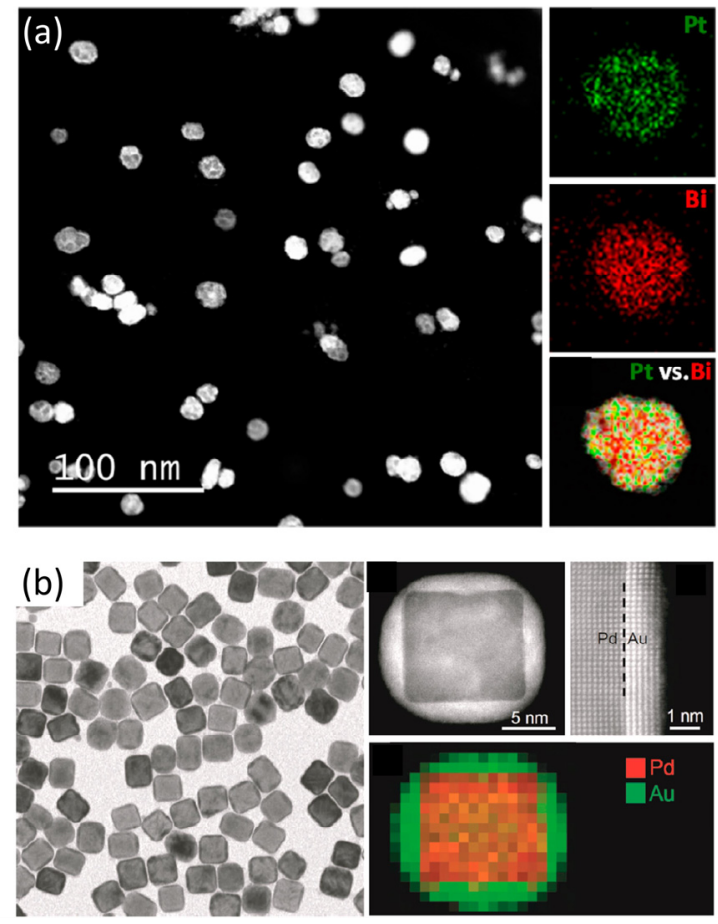

Figure 2.2. (a) HAADF-STEM image and EDS element maps of PtBi intermetallic NPs. ${ }^{70}(b)$ TEM image, HAADF-STEM image and EDX mapping of Pd-Au core-shell nanocrystals obtained using seeded growth of Au on the $18 \mathrm{~nm}$ Pd cubes. ${ }^{66}$

\section{Bimetallic NPs, Quantum Dots, Silica Particles and Zeolites Synthesis}

An overview of the synthesis of other kinds of particles such as: bimetallic NPs, quantum dots (QDs), silica microparticles and zeolites is briefly mentioned but not comprehensively summarized in Table 2.3.

Bimetallic NPs have unique properties due to their synergetic effect on catalytic reactions. By combining two metals or metal oxides the properties of the final NP can differ from the pure metal NP of the initiators. Bimetallic NPs synthesis is performed by mixing both salt solutions, providing a mixed alloy, Figure 2.2a, or by controlling the deposition rate of a second metal on a seed from the first one, Figure 2.2b.

QDs are semiconductor particles smaller than $10 \mathrm{~nm}$ in diameter and their unique optical properties are very dependent on their size. ${ }^{88}$ They are normally made of $\mathrm{CdSe}, \mathrm{CdS}, \mathrm{InP}$ or $\mathrm{PbS}$ and have been extensively synthesised using microfluidics. QDs such as CdSe are always synthesised at high temperatures which can be very well controlled in microreactors, Table 2.3. Recently, a 6-step (microfluidic chip) procedure was used to fabricate different types of core-shell QDs in a very controllable and reproducible manner. ${ }^{89}$ 
To prevent aggregation of the catalytic NP dispersion inside the channel, catalyst supports are used to stabilize the NPs. These supports are frequently made of silica, alumina or titania and they need to be very stable at high temperatures and pressures. Furthermore, they are often very porous to increase the available surface area. Various studies have synthesised porous silica micro-particles ${ }^{82-85}$, however, not with a catalytic purpose.

Zeolites are another class of commonly used catalysts, due to their nanoporosity and their acidity. However, very few studies have tried to implement their synthesis in microfluidics. Indeed, depending on the type of zeolite, the synthesis conditions can vary from room temperature to high temperatures (typically $200{ }^{\circ} \mathrm{C}$ for the reaction and more for the calcination step ${ }^{90}$ ). In zeolite synthesis precise control of the synthesis conditions is important, because there are many different types of zeolites in terms of their structure. A small change in the conditions may change the structure. That is why zeolites have been synthesized at the sub-millimeter range in microfluidics chips in droplets, ${ }^{82,83,85,91}$ but only the synthesis at relatively low temperature and short reaction time were investigated so far. The catalytic activity of these microfluidic-synthesized zeolites has been shown to decrease the reaction time of alkyl borate synthesis by a factor ten. ${ }^{85}$

\subsubsection{Conclusions}

Catalyst NPs have been extensively synthesised using microfluidics. The shape and size of the particles are very dependent on the contact form and time between the different reagents. However, few studies were found where they synthesised bimetallic NPs, zeolites and catalyst supports. It has been demonstrated that microfluidic synthesis of nanostructures provides a more uniform and reproducible approach. However, low throughput seems to be the main drawback hindering the widespread adaption by industry.

\subsubsection{Future perspectives on particle synthesis}

As stated before, using microfluidics for particle synthesis is far from its full potential. This section tries to foresee the future paths of microfluidics as a tool to synthesise catalysts NPs and other catalytic structures such as zeolites or catalyst supports.

\section{Particle Size and Structure}

Although it makes the mixing slightly more challenging, it is usually beneficial to work with smaller volumes to enhance the control over the experimental conditions. ${ }^{92}$ Microfluidic droplet-based synthesis could therefore be improved by using smaller droplets: from the typical nanoliter droplets (diameter $100 \mu \mathrm{m}$ range to the picoliter range (diameter $10 \mu \mathrm{m}$ ) and even to the femtoliter range (diameter $1 \mu \mathrm{m}$ ). Different devices have been developed to 
produce picoliter droplets such as step emulsification, ${ }^{93}$ or the so-called nebulator. ${ }^{94}$ The typical microfluidic tools for droplet control can also be miniaturized to manipulate these picoliter droplets. ${ }^{95}$ The main challenge for the miniaturization arises from the modification of the balance between surface and bulk forces: decreasing the volume of spherical droplets by a factor $10^{3}$ leads to a decrease of the volume forces by the same factor but the surfaces forces by a factor $10^{2}$ only.

Bimetallic NPs have been shown to have a great potential for catalysis. It is known that the structure and the arrangement of the two metals have a great impact on the catalytic properties of such NPs, ${ }^{96}$ and that very precise assembly is possible when the synthesis conditions are controlled. ${ }^{97}$ Therefore, the use of calibrated microfluidic droplets as microreactors could enable a very fine control on the synthesis conditions and hence on the final size and structure of such particles to optimize their catalytic activity.

Zeolites used in the industry often require high temperatures and long reaction times and their synthesis has not been fully investigated in microfluidics yet. Moreover, the model conditions offered by microfluidics could enable a precise study of the effect of mass and heat transfer on the zeolite structure that could be later used to optimize batch synthesis.

A controlled porosity of the catalyst support is essential to enable an increase of the contact area with the reaction media while preserving an efficient mass transfer through the pores. The control of substrate porosity can also be further improved by implementing microfluidics methods that have been shown efficient for bulk materials. A first method to produce such a porous media consists of triggering a chemical reaction which produces gas and solidify the reaction media. ${ }^{98}$ Despite its simplicity, this method offers poor control on the pore size and structure. The second method presented here is the use of NPs as a template that are later dissolved to pattern the material. ${ }^{99}$ In this approach, the pores consist of the space that was previously occupied by the particles, and is therefore easy to control. Compact assembly of NPs can indeed be produced inside droplets to get spherical assembly of controlled size and morphology. ${ }^{100}$ This approach seems promising to yield microparticles with controlled porosity and high contact area with the external media. The third approach presented is based on complex liquids that can be solidified during the process of phase separation to obtain a material exhibiting a continuous network of pores of controlled size. ${ }^{101}$ The conditions of phase separation leading to continuous pore networks, referred as "spinodal decomposition", has been implemented in microfluidic devices to produce porous fibres and particles stabilized by NPs of silica adsorbing at the interface. ${ }^{102}$ Such binary systems, called "bijels", experience a phase separation and are stabilized by NPs. The bottom-up approaches that rely on particle assembly or phase separation require a high level of control over the chemical and physical 
conditions. These conditions must indeed be homogeneous and reproducible, which calls for smaller reactors, as microfluidics can offer.

\section{High Throughput Synthesis}

A usual bottleneck for microfluidic devices is their throughput: smaller sizes bring better control but also naturally lower volumes produced. One of the main challenges of miniaturization is therefore to maintain the throughput. This is usually achieved by scaling out, parallelizing, the devices for droplet production and analysis.

Parallel microfluidic channels tend to interact with each other due to the variation of the pressure along the channels, ${ }^{103}$ leading to varying flow rates and non-uniform droplet generation. However, chips have been recently developed to parallelize the production of droplets with a good mono-dispersity by minimizing the flow-rates variation ${ }^{104}$ or by using emulsification processes that do not depend on flow rate. ${ }^{105-107}$ With this technology, relatively large quantities of catalyst could be synthesized with an optimal control on the reaction conditions, which could be used to synthesise NPs or catalyse reactions in microfluidic reactors.

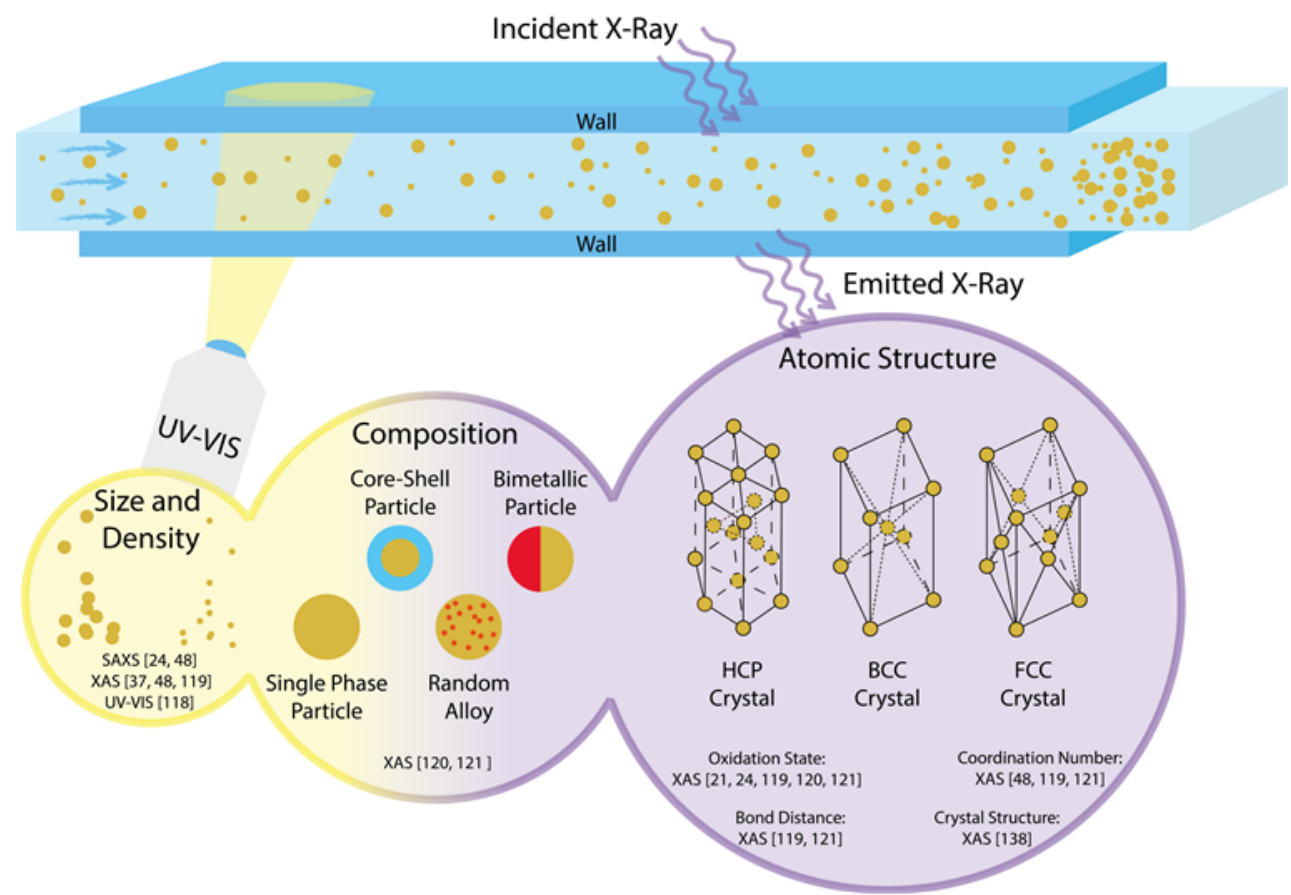

Figure 2.3. Schematic overview of in-situ characterization techniques for catalyst nanoparticles synthesis, as found in literature, using UV-Vis spectroscopy and X-ray absorption spectroscopy (XAS). 


\subsection{Characterization}

\subsubsection{Introduction}

In microfluidic synthesis, in-situ (direct) characterization can act as a fast feedback mechanism on important synthesis parameters such as flow rate and temperature. Examples of the additional parameters that determine the success rate of NP synthesis are size, shape, uniformity, oxidation state, activity and purity. In-situ measurements allow for information at different stages in both time and space during synthesis or activity testing of catalyst materials. With this information, the growth rate, reaction intermediates and deactivation can be monitored ${ }^{21,24,37,48,108-112}$ allowing for kinetic and mechanistic studies, ${ }^{108,113-117}$ as is summarized in Table 2.4 and illustrated in Figure 2.3.

To narrow down the scope of this section, only in-situ characterization will be discussed and it is divided into three parts. First, characterization techniques used in microfluidics will discussed. Second, direct characterization of NPs will be discussed, where the characterization of shape, size, oxidation state, crystal structure and elemental composition are analysed, ${ }^{21,24,37,48,118-121}$ as can be found in Figure 2.3. Last, the activity of these NPs supported in porous materials forming packed-bed or wall-coated microreactors will be treated. Catalyst NPs are often supported on porous oxides or polymers to provide stability and prevent sintering of NPs. In the characterization of supported catalyst particles, mainly the activity and selectivity of the catalyst material is of interest. Furthermore, the dispersion and stability over time of the catalyst in the support are important.

An overview of the characterization methods found in literature is shown in Table 2.4. Here, the type of reactor and the nanoparticle/support combination is listed together with the type of study, characterization and the temperature reported.

\subsubsection{Characterization techniques}

\section{Gas Chromatography and Mass Spectrometry}

To characterize the performance of a catalyst, often a reaction is monitored by looking at product formation with in-line Gas Chromatography (GC) or Mass Spectrometry (MS). With these techniques, as summarized in Table 2.4, the conversion and selectivity of a catalyst bed can be determined.108,111,115,116,122-128 The main advantage of MS and GC lies in the low limit of detection and broad range of sensitivity for multiple compounds. Unexpected products or molecules can be detected, since these in-line systems do not require tuning of the system in order to detect a specific molecule or compound. However, these in-line methods are less suitable for monitoring the catalyst bed at various times or distances along the bed. This means that in combination with microfluidics they can only be used at the outlet of a system and cannot give information about on-chip processes. Moreover, short-lived 
intermediates cannot be detected with GC and MS. For this a broad host of microscopic and spectroscopic techniques, as summarized in Table 2.4, is available i.e. Ultraviolet-Visible (UV-VIS), Infrared (IR), Raman Spectroscopy (RS), Nuclear Magnetic Resonance (NMR), and X-Ray Absorption Spectroscopy (XAS). ${ }^{109,110,112,114,116,117,123,126,129-135}$ Furthermore, it is found that GC and MS is used in combination with IR or XAS, $108,116,126,128$ as summarized in Table 2.4, to get information on both the composition of the product feed at the outlet and on the intermediates that are being formed or structural changes in the catalyst that occur from XAS and IR measurements.

\section{Nuclear Magnetic Resonance}

The advantage of nuclear magnetic resonance (NMR) is that it can give very detailed and specific information on organic compounds (H or C-NMR) or the coordination of an inorganic material (Al-NMR). However, due to the tuning of the NMR magnet, only one element can be measured at the same time; multiple NMR devices are needed to measure two different atoms simultaneously. An example of the use of NMR in microfluidic systems is given. ${ }^{112,130}$ Here hyperpolarized hydrogen is used to polarize the reactant (propane in this case). $\mathrm{H}_{2}$-polarized propane has a signal-to-noise ratio 300 times larger than non- $\mathrm{H}_{2}$-polarized propylene. ${ }^{112}$ This was used to monitor the formation of propane from propylene.

\section{Ultraviolet-Visible Spectroscopy and X-Ray Absorption Spectroscopy}

In many photocatalytically-active particle studies, the degradation of a catalyst by the de-colouring of a dye is studied. This can either be measured with a change in the UV-VIS absorption spectrum ${ }^{109,110,114,132}$ or by looking directly at the intensity of the emitted light with a photometer. 113,136 The advantage of UV-VIS is that it is easily performed in combination with microfluidics. Transparent glass-glass reactors or tube reactors are suitable in combination with UV-VIS, 109,110,114,132,135 as summarized in Table 2.4. The limitation here is determined by the absorption of UV-light by the glass cover, which fully absorbs wavelengths below $250 \mathrm{~nm}^{137}$.

X-Ray Absorption Spectroscopy (XAS) and in particular Extended X-Ray Absorption at Fine Structures (EXAFS) and X-Ray Absorption Near Edge Structures (XANES) are very powerful tools in characterizing composition, bond distance, oxidation state, coordination number and crystal structure of NPs, ${ }^{21,24,37,48,108,119-121,138}$ as shown in Figure 2.3. Disadvantages of XAS measurements are the need for an extensive database of reference samples, and both the time-consuming and complex data analysis that is needed to extract the desired information. Still, it can be noticed that microfluidics is much used, and actually required in XAS measurements due to the requirement of small volumes and reactor dimensions in synchrotron facilities. As example, the microreactor used by Gross et al. 2014, ${ }^{116}$ benefits from the micrometre-range 
channel heights, as less of the X-Ray intensity is lost in XAS transmission mode. Furthermore, the spatial resolution of $15 \mu \mathrm{m}$ that can be achieved with synchrotron measurements can be used to measure along multiple points in the microfluidic channels. For UV-VIS it is not strictly necessary to use small volumes and reactors, although it is still an advantageous technique to combine with microfluidics, ${ }^{61,109,110,114,132,135}$ as summarized in Table 2.4. Furthermore, UVVIS is a much more accessible technique, since no synchrotron facilities are required to perform measurements. Figure 2.4a shows an example of a tube reactor coated with $\mathrm{ZnO}$ nanorods for the photocatalytic degradation of Methylene Blue (MB). The obtained absorption spectra are shown in Figure 2.4b Besides monitoring the formation and growth of nanoparticles, XAS can also be used to monitor the state of catalysts during operation. Change in chemical state, crystal structure and oxidation state give information about the performance of the catalyst in terms of deactivation and the mechanism of the reaction, $108,111,116,128$ as summarized in Table 2.4. This information is of importance because the deactivation is a common phenomenon in catalysis. ${ }^{139}$ More about catalyst deactivation will be discussed later in section 2.3.4.

(a)

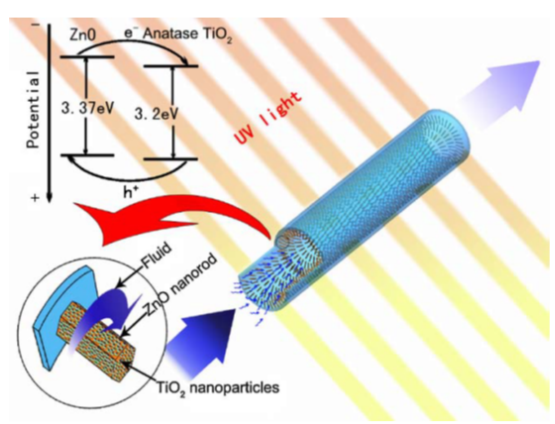

(b)

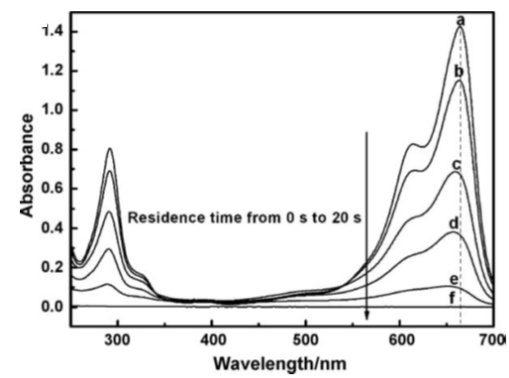

Figure 2.4. (a) Schematic diagram of the continuous-flow photocatalysis and the interfacial charge transfer between coupled $\mathrm{ZnO}$ nanorods and $\mathrm{TiO}_{2}$ nanoparticles. (b) Absorption spectra changes of the $\mathrm{MB}$ solution degraded by being pumped into the TiO2/ZnO-3 CM at different RTs under the irradiation of a UV lamp. RT of the curves are (a) $0 \mathrm{~s},(b) 1 \mathrm{~s},(\mathrm{c}) 3 \mathrm{~s},(\mathrm{~d}) 5 \mathrm{~s},(\mathrm{e})$ $10 \mathrm{~s}$ and $(f) 20 \mathrm{~s}$. Images taken from ${ }^{110}$.

\section{Infrared and Raman Spectroscopy}

Attenuated Total Reflection-Infrared (ATR-IR) and Fourier Transformed Infrared (FTIR) can also be used to monitor reaction intermediates and product formation, $108,116,126,131$ see Table 2.4. Furthermore, Figure 2.5a shows the reactor and Figure 2.5b the data obtained as an example of the use of IR spectroscopy, where reactants and products can be monitored in flow in a microfluidic reactor. One of the difficulties of ATR-IR lies in the chip design and the total internal reflection of an IR beam in the ATR crystal. The evanescent wave going 
into the sample has a small penetration depth. Therefore, the chip design has to be such that the channels are in close contact with the ATR-IR crystal. This is possible with silicon micromachining as shown in a review by Karbudak et al. in 2014, ${ }^{140}$ enabling catalyst film characterization using ATR-IR in combination with microfluidic devices. Furthermore, the adsorption of gas to a catalyst film can be measured using ATR-IR.133 Water is incompatible with the use of IR techniques because it has a very broad IR signal and will likely dominate the spectrum. A technique that allows to work with water is Raman spectroscopy (RS). However, this technique comes with its own disadvantages: spontaneous inelastic scattering typically only happens once every $10^{6}$ photons. This makes RS signal very weak, although there are ways to enhance the signal e.g. by using surface enhancement structures. ${ }^{117,141,142}$ As example, the catalytic reduction of p-Nitrothiophenol (PNTP) on gold NPs was performed and measured using Surface Enhanced Raman Spectroscopy (SERS). ${ }^{117}$ Figure 2.6a and b show the reactor used in the catalytic reduction of PNTP and the SERS spectrum obtained, respectively. With SERS they were able to calculate the rate constant for the PNTP reduction, see Figure 2.6c.

(a)

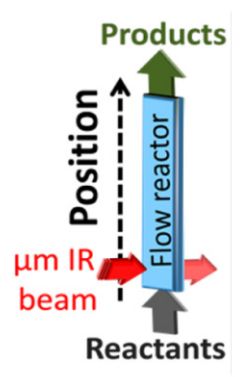

(b)

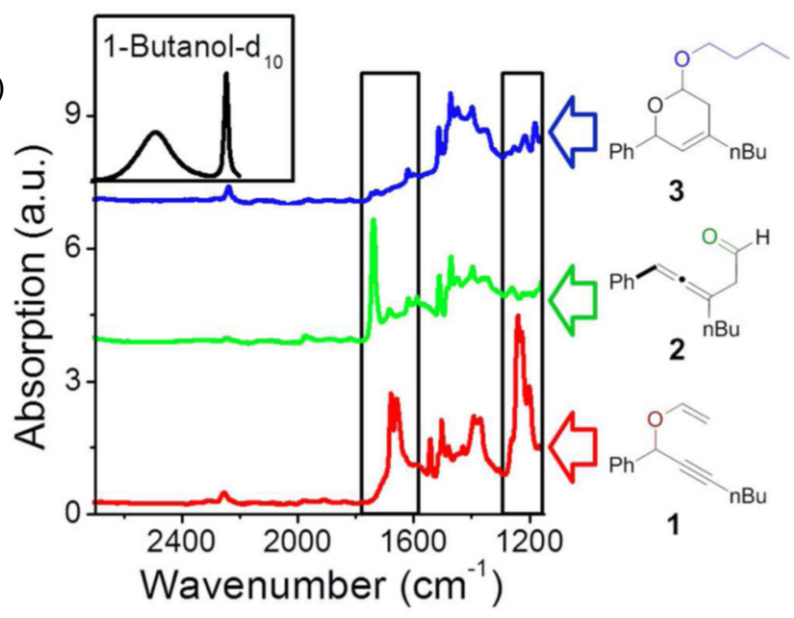

Figure 2.5. (a) A scheme of the micro-IR flow reactor and (b) the FTIR spectra of the reactant, vinyl ether 1 (red), the primary product, allenic aldehyde 2 (green), and the secondary product, acetal 3 (blue). High-energy regime of IR absorption spectra of butanol-d10 (black), the second reactant, is shown as well. The black rectangles mark the areas in which the IR spectra of the different reactants and products can be easily distinguished from each other. Images taken from $^{116}$ 


\subsubsection{In-situ Characterization of Nanoparticles in Microfluidic Systems}

For in-situ characterization of nanoparticles, it is most important to characterize properties such as size, density, composition and atomic structure, as shown in Figure 2.3. XAS is required especially for the composition and atomic structure of NPs. With XAS it is possible to probe single atoms and atom clusters. Another advantage of XAS in the characterization of synthesized NPs is the great time resolution that can be achieved. As example, XANES was used to monitor the reduction of $\mathrm{Au}^{+3}$ to metallic gold within the first $2 \mathrm{~ms}$ of the formation. ${ }^{21}$ With EXAFS the growth rate can be monitored by measuring the evolution of size of the formed NPs over time. ${ }^{21,48}$

(a)

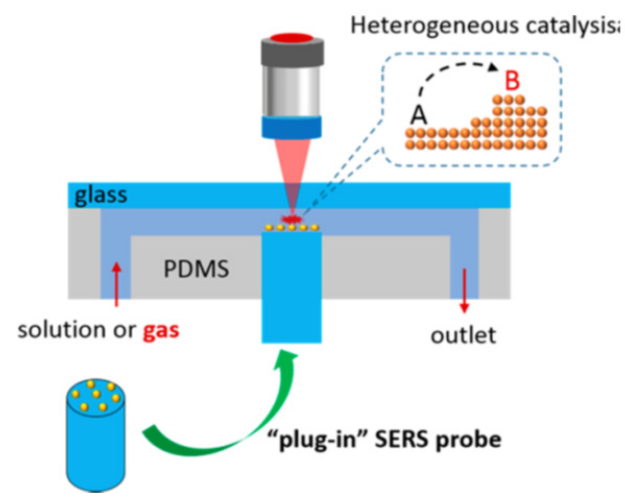

(b)

(c)
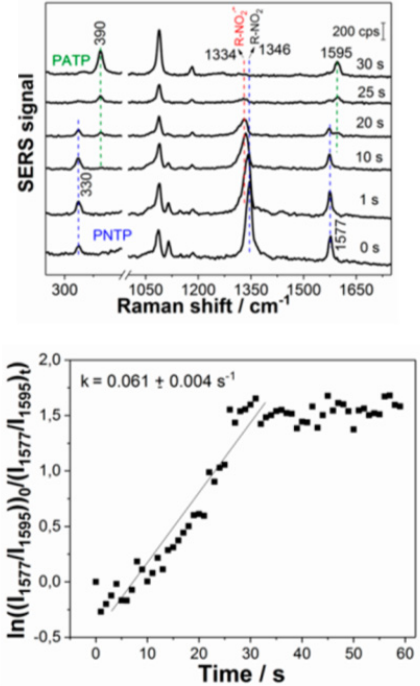

Figure 2.6. (a) Schematic of the whole plug-in optofluidic platform for monitoring of nanoparticlecatalyzed reactions in a microfluidic platform. (b) SERS spectra recorded during the catalytic reduction of PNTP on immobilized gold nanoparticles in an aqueous $\mathrm{NaBH} 4$ solution at different times. (c) Determination of the rate constant for the reduction of PNTP using the relative concentration of PNTP and PATP. Quantification is achieved by comparing the intensities of their characteristic bands at 1577 and $1595 \mathrm{~cm}-1$ (גexcitation of $633 \mathrm{~nm}$, intensity of $3 \mathrm{~kW} / \mathrm{cm}^{2}$, acquisition time of 1 s). Images taken from ${ }^{117}$

An alternative to study the size of the synthesized NPs is UV-VIS, ${ }^{118}$ see Figure 2.3. The plasmon absorption peak observed in UV-VIS analysis is dependent on the size of the nanoclusters. ${ }^{24}$ In order to characterize size and shape also Small Angle X-Ray Scattering (SAXS) can be used. ${ }^{118}$ It is shown that in general UV-VIS data for size characterization leads to more uncertainties than SAXS analysis. However, UV-VIS is much easier to use in comparison to SAXS experiments which need synchrotron facilities, as mentioned before. Furthermore, it was demonstrated that the formation of atomically precise 1-2 
nm-sized copper clusters could be detected using UV-VIS, although no-flow conditions were used. ${ }^{119}$ This shows the potential to use UV-VIS in size characterization of NPs. ${ }^{118}$

Table 2.4. Some properties of in-situ characterization of catalysts using microfluidics. For a list of used acronyms, refer to the end of the chapter.

\begin{tabular}{|c|c|c|c|c|c|}
\hline $\begin{array}{l}\text { Catalyst } \\
\text { support } \\
\text { (Reactor type) }\end{array}$ & Measured property & $\begin{array}{l}\text { Analytical } \\
\text { technique }\end{array}$ & Temp & $\begin{array}{l}\text { Reactor } \\
\text { material }\end{array}$ & Ref \\
\hline $\begin{array}{l}\text { Pt/PMMA- } \\
\text { polyDADMA } \\
\text { C (packed- } \\
\text { bed) }\end{array}$ & $\begin{array}{l}\text { Degradation of Cochenille Red } \\
\text { with sodiumborohydride }\end{array}$ & $\begin{array}{l}\text { Optical/VIS: } \\
\text { photometer }\end{array}$ & $\begin{array}{l}25- \\
80^{\circ} \mathrm{C}\end{array}$ & $\begin{array}{l}\text { Glass } \\
\text { microtube }\end{array}$ & 67 \\
\hline $\begin{array}{l}\mathrm{Pt} / \mathrm{SiO}_{2} \\
\mathrm{Au} / \mathrm{TiOSilicon-} \\
\text { glass chip } \\
\mathrm{Au} / \mathrm{Al}_{2} \mathrm{O}_{3} \text { (wall } \\
\text { coated) }\end{array}$ & $\begin{array}{l}\text { Bonding state and chemical state } \\
\text { of } \mathrm{Au} / \mathrm{TiO}_{2}(\mathrm{XANES}) \text { - } \\
\text { Coordination numbers and bond } \\
\text { lengths for } \mathrm{Au} / \mathrm{Al}_{2} \mathrm{O}_{3}(\mathrm{EXAFS} \text { - } \\
\text { Crystal structure of } \mathrm{TiO}_{2} \text { support } \\
\text { in } \mathrm{Au} / \mathrm{TiO}_{2} \text { (EXAFS) - } \\
\text { Intermediates and products in } \\
\text { ethylene hydrogenation (IR and } \\
\text { GC) }\end{array}$ & $\begin{array}{l}\text { XANES, } \\
\text { EXAFS, IR } \\
\text { and GC }\end{array}$ & $\begin{array}{l}40-200 \\
{ }^{\circ} \mathrm{C}\end{array}$ & $\begin{array}{l}\text { Silicon- } \\
\text { silicon chip } \\
\text { with } \\
\text { siliconnitrid } \\
\text { e windows }\end{array}$ & 108 \\
\hline $\begin{array}{l}\mathrm{Pt} / \mathrm{ZnO} \\
\text { nanorod (wall } \\
\text { coated) }\end{array}$ & $\begin{array}{l}\text { Photocatalytic activity measured } \\
\text { with the degradation of phenol }\end{array}$ & UV-VIS & NS & $\begin{array}{l}\text { Fused-silica } \\
\text { capillary }\end{array}$ & 109 \\
\hline $\begin{array}{l}\mathrm{ZnO} / \mathrm{TiO}_{2} \\
\text { (wall coated) }\end{array}$ & $\begin{array}{l}\text { Photocatalytic kinetic } \\
\text { performance with the } \\
\text { degradation of methylene blue }\end{array}$ & UV-VIS & NS & $\begin{array}{l}\text { Fused-silica } \\
\text { capillary }\end{array}$ & 110 \\
\hline $\begin{array}{l}\mathrm{Rh} / \mathrm{Al}_{2} \mathrm{O}_{3} \\
\text { (wall-coated) }\end{array}$ & $\begin{array}{l}\text { Oxidation state of Rh during } \\
\text { partial oxidation of methane } \\
\text { (XANES) - Selectivity and } \\
\text { conversion for direct partial } \\
\text { oxidation of methane (MS) }\end{array}$ & $\begin{array}{l}\text { XANES and } \\
\text { MS }\end{array}$ & $\begin{array}{l}331- \\
378^{\circ} \mathrm{C}\end{array}$ & $\begin{array}{l}\text { Quartz } \\
\text { capillary }\end{array}$ & 111 \\
\hline $\begin{array}{l}\mathrm{Pt} / \mathrm{Al}_{2} \mathrm{O}_{3} \text { and } \\
\mathrm{Pd} / \mathrm{Al}_{2} \mathrm{O}_{3} \\
\text { (packed-bed) }\end{array}$ & $\begin{array}{l}\text { Propane formation from } \\
\text { propylene and para-hydrogen - } \\
\text { Density of catalyst sites }\end{array}$ & NMR & NS & $\begin{array}{l}\text { Capillary } \\
\text { (not } \\
\text { specified) }\end{array}$ & 112 \\
\hline $\begin{array}{l}\mathrm{M}-\mathrm{TiO}_{2}(\mathrm{M}=\mathrm{F}, \\
\mathrm{Si}, \mathrm{K}, \mathrm{Mn}, \mathrm{Co}, \\
\mathrm{Ni}, \mathrm{Cu}, \mathrm{Mo}, \\
\text { and } \mathrm{Au}) \\
\text { (packed-bed) }\end{array}$ & $\begin{array}{l}\text { Photocatalytic activity measured } \\
\text { with the degradation of } \\
\text { methylene blue }\end{array}$ & $\begin{array}{l}\text { Optical/VIS: } \\
\text { CCD camera }\end{array}$ & NS & Glass-glass & 113 \\
\hline $\begin{array}{l}\mathrm{TiO}_{2} \text { (wall- } \\
\text { coated) }\end{array}$ & $\begin{array}{l}\text { Photocatalytic activity measured } \\
\text { with the degradation of } \\
\text { Rhodamine B }\end{array}$ & UV-VIS & NS & $\begin{array}{l}\text { Fused-silica } \\
\text { capillary }\end{array}$ & 114 \\
\hline $\begin{array}{l}\mathrm{Ru} \\
\text { Nanoparticles } \\
\text { (wall-coated in } \\
\text { polymeric } \\
\text { matrix) }\end{array}$ & $\begin{array}{l}\text { Catalytic activity by } \\
\text { hydrogenation of various } \\
\text { carbonyl compounds }\end{array}$ & GC-MS & $\begin{array}{l}<160 \\
{ }^{\circ} \mathrm{C}\end{array}$ & $\begin{array}{l}\text { Fused-silica } \\
\text { capillaries }\end{array}$ & 115 \\
\hline $\begin{array}{l}\mathrm{Au} / \mathrm{SiO}_{2} \\
\text { (packed-bed) }\end{array}$ & $\begin{array}{l}\text { Cascade dihydropyran synthesis, } \\
\text { yield and selectivity (IR and GC) } \\
\text { - Oxidation state indicating } \\
\text { catalyst deactivation (EXAFS) }\end{array}$ & $\begin{array}{l}\text { IR, GC, and } \\
\text { EXAFS }\end{array}$ & NS & $\begin{array}{l}\text { Stainless } \\
\text { steel } \\
\text { plugflow } \\
\text { reactor }\end{array}$ & 116 \\
\hline
\end{tabular}




\begin{tabular}{|c|c|c|c|c|c|}
\hline $\begin{array}{l}\text { Au } \\
\text { Nanoparticles } \\
\text { (wall-coated) }\end{array}$ & $\begin{array}{l}\text { Reduction of p-nitrothiophenol - } \\
\text { Influence of } \mathrm{O}_{2} \text { and } \mathrm{H}_{2} \text { on } \\
\text { dimerization of p- } \\
\text { aminothiophenol }\end{array}$ & SERS & NS & $\begin{array}{l}\text { Glass- } \\
\text { PDMS }\end{array}$ & 117 \\
\hline $\begin{array}{l}\mathrm{Cu} / \mathrm{ZnO} \\
(\text { packed bed) }\end{array}$ & $\begin{array}{l}\text { Activity by methanol to } \\
\text { hydrogen conversion }\end{array}$ & GC & $\begin{array}{l}195- \\
260^{\circ} \mathrm{C} \\
\end{array}$ & $\begin{array}{l}\text { Silicon-glass } \\
\text { chip }\end{array}$ & 122 \\
\hline $\begin{array}{l}\mathrm{Pt}-\mathrm{Co} / \mathrm{Al}_{2} \mathrm{O}_{3} \\
\text { (wall-coated) }\end{array}$ & $\begin{array}{l}\text { Oxidation state of } \mathrm{Co} \text { and } \mathrm{Co} \\
\text { oxides (Raman) - } \mathrm{CO} \text { conversion, } \\
\mathrm{O}_{2} \text { conversion and } \mathrm{CO}_{2} \text { selectivity } \\
\text { for preferential oxidation of } \\
\text { carbonmonoxide(GC) }\end{array}$ & $\begin{array}{l}\text { Raman and } \\
\text { GC }\end{array}$ & $\begin{array}{l}120- \\
160^{\circ} \mathrm{C}\end{array}$ & $\begin{array}{l}\text { Fe-Cr-Al } \\
\text { capillary in } \\
\text { a stainless } \\
\text { steel tube }\end{array}$ & 123 \\
\hline $\begin{array}{l}\text { Pt thin film } \\
(\text { catalyst spot } \\
\left.15 \mu \mathrm{m}^{2}\right)\end{array}$ & $\begin{array}{l}\text { Catalytic activity by } \mathrm{CO} \\
\text { oxidation }\end{array}$ & MS & $\begin{array}{l}<300 \\
{ }^{\circ} \mathrm{C}\end{array}$ & Silicon-glass & 124 \\
\hline $\begin{array}{l}\mathrm{Cu}_{\mathrm{x}} \mathrm{Pd}_{\mathrm{y}} \mathrm{Au}(1-\mathrm{x}-\mathrm{y}) \\
\text { alloy surface } \\
\text { (wall-coated) } \\
\end{array}$ & $\begin{array}{l}\text { Catalytic activity by } \mathrm{H}_{2}-\mathrm{D}_{2} \\
\text { exchange reaction }\end{array}$ & MS & $\begin{array}{l}27-327 \\
{ }^{\circ} \mathrm{C}\end{array}$ & $\begin{array}{l}\text { Glass-glass } \\
\text { chip }\end{array}$ & 125 \\
\hline $\begin{array}{l}\mathrm{Pd} / \mathrm{Al}_{2} \mathrm{O}_{3} \\
\text { (packed-bed) }\end{array}$ & $\begin{array}{l}\text { Turn over frequency (TOF) for } \\
\text { oxidation of Carbon monoxide }\end{array}$ & $\begin{array}{l}\text { FTIR and } \\
\text { GC }\end{array}$ & $250^{\circ} \mathrm{C}$ & Silicon-glass & 126 \\
\hline $\begin{array}{l}\mathrm{Pt} / \mathrm{Al}_{2} \mathrm{O}_{3} \text { and } \\
\mathrm{Rh} / \mathrm{Al}_{2} \mathrm{O}_{3} \\
\text { (fixed-bed) }\end{array}$ & $\begin{array}{l}\text { Deactivation by monitoring } \\
\text { oxidation state during reaction } \\
\text { (XANES) } \\
\text { Conversion of partial oxidation } \\
\text { of methane (MS) }\end{array}$ & $\begin{array}{l}\text { XANES and } \\
\text { MS }\end{array}$ & $352{ }^{\circ} \mathrm{C}$ & $\begin{array}{l}\text { Quartz } \\
\text { glass } \\
\text { capillary }\end{array}$ & 128 \\
\hline $\begin{array}{l}\text { Au-Pd/TiO } 2 \\
\text { (packed-bed) }\end{array}$ & $\begin{array}{l}\text { Conversion and selectivity for } \\
\text { the oxidation of benzyl alcohol }\end{array}$ & Raman & $\begin{array}{l}80-140 \\
{ }^{\circ} \mathrm{C}\end{array}$ & $\begin{array}{l}\text { Silicon-glass } \\
\text { chip }\end{array}$ & 129 \\
\hline $\begin{array}{l}\mathrm{Rh} / \mathrm{SiO}_{2} \\
\text { (packed-bed) }\end{array}$ & $\begin{array}{l}\text { Turn over frequency (TOF) for } \\
\text { propene hydrogenation with } \\
\text { hyperpolarized Hydrogen }\end{array}$ & NMR & $60^{\circ} \mathrm{C}$ & $\begin{array}{l}\text { Capillary } \\
\text { (not } \\
\text { specified) }\end{array}$ & 130 \\
\hline $\begin{array}{l}\text { Pd/Carbon } \\
\text { and Zeolite Y } \\
\text { and Beta } \\
\text { (multiple } \\
\text { particles in } \\
\text { droplet) } \\
\end{array}$ & Yield for the acylation of anisole & ATR-IR & $\begin{array}{l}{ }^{20-150} \\
{ }^{\circ} \mathrm{C}\end{array}$ & PFA tubing & 131 \\
\hline $\begin{array}{l}\text { PtNi } \\
\text { Nanoparticles } \\
\text { (wall-coated) } \\
\end{array}$ & $\begin{array}{l}\text { Hydrogenation of azobenzene } \\
\text { dye }\end{array}$ & UV-VIS & NS & $\begin{array}{l}\text { Photonic } \\
\text { crystal fiber }\end{array}$ & 132 \\
\hline $\begin{array}{l}\text { Pt film (wall- } \\
\text { coated) }\end{array}$ & Adsorption of CO on Pt film & ATR-IR & NS & $\begin{array}{l}\text { Stainless } \\
\text { steel tube }\end{array}$ & 133 \\
\hline $\begin{array}{l}\text { Au-Pt-Au NPs } \\
\text { (suspension) }\end{array}$ & $\begin{array}{l}\text { Reduction of 4-nitrothiophenol } \\
\text { by adsorbed hydrogen }\end{array}$ & SERS & $\begin{array}{l}{ }^{5-50} \\
{ }^{\circ} \mathrm{C}\end{array}$ & Glass-glass & 134 \\
\hline $\begin{array}{l}\text { Au-Ag alloy } \\
\text { NPs (wall- } \\
\text { coated) }\end{array}$ & $\begin{array}{l}\text { Reduction of 4-nitrophenol for } \\
\text { kinetic study of various } \mathrm{Au} / \mathrm{Ag} \\
\text { ratios }\end{array}$ & UV-VIS & NS & Silicon-glass & 135 \\
\hline $\begin{array}{l}\mathrm{TiO}_{2} \text { graphene } \\
\text { oxide (wall- } \\
\text { coated) }\end{array}$ & $\begin{array}{l}\text { Photocatalytic activity measured } \\
\text { with the degradation of } \\
\text { Methylene Blue }\end{array}$ & $\begin{array}{l}\text { Optical/VIS: } \\
\text { absorbance } \\
\text { through } \\
\text { channel }\end{array}$ & NS & $\begin{array}{l}\text { PDMS- } \\
\text { PDMS }\end{array}$ & 136 \\
\hline $\begin{array}{l}\mathrm{Pt} / \mathrm{Al}_{2} \mathrm{O}_{3} \\
\text { (packed-bed) }\end{array}$ & $\begin{array}{l}\mathrm{Pt} \text { dispersion in } \mathrm{Pt} / \mathrm{Al}_{2} \mathrm{O}_{3} \text { catalyst } \\
\text { layer }\end{array}$ & $\begin{array}{l}\mathrm{CO} \\
\text { chemisorptio } \\
\mathrm{n} \text { and GC }\end{array}$ & $\begin{array}{l}25-250 \\
{ }^{\circ} \mathrm{C}\end{array}$ & $\begin{array}{l}\text { Silicon-glass } \\
\text { chip }\end{array}$ & $\begin{array}{l}{ }^{143} \mathrm{y} \\
\mathrm{y}\end{array}$ \\
\hline
\end{tabular}




\subsubsection{Activity of supported catalyst nanoparticles in microreactors}

Activity measurements of catalysts in microfluidic devices allow the study of catalyst material under relevant conditions with well-controlled parameters such as temperature, flow rate, and pressure, see Table 2.4. As an example, it was previously reported that microfluidic reactors are capable of operating at temperatures and pressures up to $500{ }^{\circ} \mathrm{C}$ and 200 bar. ${ }^{144-146}$ Catalyst activity studies are, in contrary to the NP characterization discussed earlier, done on supported catalyst material. The most common type of reactors are packed-bed and wall coated reactors. Catalyst particles are either loaded on larger porous supports, this can be oxide or polymer particles, ${ }^{67,112,113,116,122,126,127,129,130,143}$ or deposited as thin films on the reactor walls, ${ }^{108-111,114,115,117,123-125,132,133,135,136}$ as summarized in Table 2.4.

In a packed-bed reactor, a large number of particles are used to make up the bed. This averages out the catalytic performance hindering the information at single particle level. ${ }^{126}$ Packed-bed reactors have a large pressure drop over the reactor bed, although an inventive solution was found ${ }^{126,127}$ where instead of elongating the packed-bed, shorter parallel beds were used in a so called crossflow packed-bed reactor.

Sizes of NPs supports that are used in packed-bed reactors are between 20 and 120 micron as found. ${ }^{113,129}$ Packed bed reactors have large effective areas compared to wall-coated reactors. In packed bed reactors, supported catalyst material can be placed either in a microfluidic chip, often consisting of siliconsilicon, ${ }^{108}$ glass-silicon, ${ }^{122,124,126,127,129,135,143}$ glass-glass, ${ }^{113,125,134}$ PDMS-glass, ${ }^{117}$ PDMS-PDMS, 136 or in a piece of tubing/capillary67,109-112,114-116,123,128,130-133 to form a microreactor. All the aforementioned examples are summarized in Table 2.4. Control of temperature both spatially and in time allows for a more detailed study of kinetics and is necessary to mimic relevant reaction conditions in large reactors. On-chip reactors have the advantage of incorporating mixing modules ${ }^{134}$ followed by hot reaction zones, by using heater elements, on the chip.124,144 Silicon-glass microreactors, for example, can be fabricated with integrated heaters allowing rapid and accurate control of the temperature, ${ }^{122}$ as well as steep gradients, rapid cycling, and precise local heating, however, cleanroom facilities are needed. On the other hand, capillary reactors are simple and easier to fabricate. ${ }^{67,109-112,114-116,123,128,130-133}$ However, external heaters, such as oil baths, electrical heaters or ovens, have to be used in combination with these capillary reactors. This makes the heating process slower when adjusting or cycling the temperature, and spatial control is lost to a certain degree. ${ }^{67,123-}$ $125,129,131$

\section{Accessibility of active sites and deactivation studies}

Another parameter influencing the activity of a catalyst bed is the amount and accessibility of active sites of the catalysts. CO chemisorption, an often used bulk technique to determine acidity, was implemented in a microfluidic device 
for the quantitative characterization of alumina-supported Pt NPs in a highthroughput fashion. ${ }^{143}$ Due to the strong chemisorption of CO on metal NPs, the Pt distribution in the catalyst could be determined using a silicon microfabricated packed-bed reactor. It should be noted that bulk chemisorption is considered a reliable method, but difficult to perform with smaller samples.

Monitoring catalyst activity (or deactivation) over a longer period of time can be very useful. In-situ catalyst deactivation studies show the importance of catalyst characterization while they are at work. ${ }^{139,147}$ However, due to the large intrinsic heterogeneities in a catalyst sample, it is not always sufficient to look at the bulk scale. Downsizing to a single particle level is very convenient and possible using microfluidics.

A few studies also focus on long-term catalyst performance and deactivation, ${ }^{12,109-111}$ some of those studies extending over 100 and 120 h. ${ }^{109,110}$ By combining synchrotron-based IR and XAS measurement, as done by Gross et al. $2014^{116}$, reactant depletion, product formation and changes in catalyst during the reaction could be monitored.

\section{High-throughput characterization}

In order to overcome the low volume throughput of microfluidic reactors, millifluidic reactors are used (scale up), where the throughput is increased by increasing the reactor volume (increasing the channel dimensions). These reactors still have the favourable conditions of microfluidics such as: high surface-to-volume ratio, fast heat transfer, easy to vary reaction conditions and homogeneous reaction environments, but are capable of higher throughput for synthesis and characterization. 37,66,119,120,131 In addition, parallelization (scale-out) is often used in microfluidics to increase throughput. ${ }^{110,113,126,127,148}$ Here, instead of increasing the dimensions, separate reaction channels are used to screen various catalyst compositions. Clever tricks such as the cross-flow reactor, by the group of Jensen, 126,127 do not only reduce the pressure drop over the catalyst bed but also increase the throughput. Finally, a high-throughput screening platform is demonstrated where a foil forms a $\mathrm{Cu}_{x} \mathrm{Pd}_{y} \mathrm{Au}_{\{1-x-y\}}$ alloy surface with 100 isolated catalyst regions with different catalyst composition. ${ }^{125}$ Each of the regions is individually addressable and products produced at each spot after a $\mathrm{H}_{2} \mathrm{D}_{2}$ exchange reaction can be coupled into a MS. The nozzle of the MS can rapidly move between the 100 channels sampling products from all channels sequentially within 10 minutes $^{125}$, as shown in Figure 2.7.

\subsubsection{Characterization methods overview}

In Table 2.5 an overview of various characterization methods, namely infrared, UV-Vis, Raman, gas chromatography (GC)-mass spectrometry (MS), $X$-ray absorption spectroscopy (XAS), nuclear magnetic resonance (NMR), and their use in combination with microfluidics is listed. These methods have been 
explored to investigate reaction intermediates, process yields, as well as selectivity, activity and stability of catalyst materials. Key features, such as speed, spatial resolution and chemical information obtained, are listed, as well as the foreseen limitations, and their compatibility with microfluidics are stated.

Table 2.5. Overview of the properties of different characterization methods that can be used in microreactors.

\begin{tabular}{|c|c|c|c|}
\hline Key feature & Infrared & UV-Vis & Raman \\
\hline Speed $^{1}$ & $\begin{array}{l}\text { Scan speed of } 400 \mathrm{~Hz} \text { for } \\
\text { FTIR with Quantum } \\
\text { Cascade Laser }{ }^{149}\end{array}$ & Scan speed of $0.5 \mathrm{~Hz}^{150}$ & $\begin{array}{l}\text { Scan times of } 30 \mathrm{~ms} \text { for } \\
\text { Coherent Anti-Stokes } \\
\text { Raman scattering } \\
\text { (CARS) up to } 100 \mathrm{~s} 151\end{array}$ \\
\hline $\begin{array}{l}\text { Spatial } \\
\text { resolution }^{2}\end{array}$ & $\begin{array}{l}\sim 3 \mu \mathrm{m} \text { up to } \mathrm{mm} \text { range } \\
156\end{array}$ & $\begin{array}{l}\sim 1 \mu \mathrm{m} \text { up to } \mathrm{mm} \text { range } \\
156\end{array}$ & $\begin{array}{l}\sim 0,5 \mu \mathrm{m} \text { for Coherent } \\
\text { Anti-Stokes Raman } \\
\text { Scattering (CARS) }{ }^{156}\end{array}$ \\
\hline $\begin{array}{l}\text { Chemical } \\
\text { information }\end{array}$ & $\begin{array}{l}\text { Chemical compound } \\
\text { information by probing } \\
\text { intra-molecular } \\
\text { vibrations }\end{array}$ & $\begin{array}{l}\text { Chemical compound } \\
\text { information via } \\
\text { electronic transitions }\end{array}$ & $\begin{array}{l}\text { Chemical compound } \\
\text { information by } \\
\text { probing intra- } \\
\text { molecular vibrations }\end{array}$ \\
\hline Limitations & $\begin{array}{l}\text { Incompatible with water. } \\
\text { Limited to several types } \\
\text { of chemical bonds }\end{array}$ & $\begin{array}{l}\text { Incompatible with } \\
\text { borosilicate due to } \\
\text { absorption of light; } \\
\text { molecular fingerprinting } \\
\text { is difficult to broad } \\
\text { overlapping absorption } \\
\text { bands }\end{array}$ & $\begin{array}{l}\text { Low efficiency of } \\
\text { normal Raman } \\
\text { scattering (no SERS or } \\
\text { CARS) results in long } \\
\text { scanning times }{ }^{151}\end{array}$ \\
\hline Compatibility & $\begin{array}{l}\text { + Good compatibility } \\
\text { with microfluidics } \\
\text { working with water } \\
\text { makes the choice of } \\
\text { solvents more limited. }\end{array}$ & $\begin{array}{l}\text { + Good compatibility } \\
\text { with microfluidics. The } \\
\text { scan time needed for a } \\
\text { clear signal. Working in } \\
\text { flow or droplets can } \\
\text { influence this. }\end{array}$ & $\begin{array}{l}\text { + Good compatibility } \\
\text { with microfluidics. } \\
\text { Often enhancement is } \\
\text { needed to get a large } \\
\text { enough signal on chip. } \\
\text { Enhancement also } \\
\text { reduces scan speed } \\
\text { and improves } \\
\text { temporal information. }\end{array}$ \\
\hline
\end{tabular}

\begin{tabular}{|l|l|l|l|}
\hline Key feature & GC/MS & XAS & NMR \\
\hline Speed ${ }^{1}$ & $\begin{array}{l}\text { On-line analysis can go } \\
\text { up to } 650 \text { scans/s } 152\end{array}$ & $\begin{array}{l}\text { Reported speeds from } ~ \\
10 \text { fs to ms 153,154 }\end{array}$ & $\begin{array}{l}\text { Broad range of scan } \\
\text { rates possible from } \\
\text { nanosecond to second } \\
\text { range }{ }^{155}\end{array}$ \\
\hline $\begin{array}{l}\text { Spatial } \\
\text { resolution }{ }^{2}\end{array}$ & N.A. & $15 \mathrm{~nm}-15 \mu \mathrm{m}^{116,128,156}$ & $\begin{array}{l}\text { Ranging from mm } \mathrm{mm}^{130} \\
\text { to } \sim 20 \mu \mathrm{m}^{112}\end{array}$ \\
\hline $\begin{array}{l}\text { Chemical } \\
\text { information }\end{array}$ & $\begin{array}{l}\text { Chemical composition of } \\
\text { feed }\end{array}$ & $\begin{array}{l}\text { Chemical compound } \\
\text { information, via bond } \\
\text { distance, oxidation state, } \\
\text { coordination number, } \\
\text { and chemical elemental } \\
\text { information }\end{array}$ & $\begin{array}{l}\text { Chemical compound } \\
\text { information via } \\
\text { chemical shifts of } \\
\text { elements present, and } \\
\text { the relative magnetic }\end{array}$ \\
\hline
\end{tabular}




\begin{tabular}{|l|l|l|l|}
\hline Limitations & $\begin{array}{l}\text { No operando or in-situ } \\
\text { information possible. }\end{array}$ & $\begin{array}{l}\text { Synchrotron facilities } \\
\text { needed, although lab- } \\
\text { based XAS methods are } \\
\text { becoming increasingly } \\
\text { popular. }\end{array}$ & $\begin{array}{l}\text { Some nuclei require } \\
\text { long acquisition times } \\
\text { them. }\end{array}$ \\
\hline $\begin{array}{l}\text { be analyzed. } \\
\text { Compatibility } \\
\text { Minimum sample } \\
\text { volume needed }\end{array}$ & $\begin{array}{l}\text { - The need for relatively } \\
\text { large sample volumes } \\
\text { and the fact that only } \\
\text { outlet products can be } \\
\text { analyzed make it not } \\
\text { always useful, although } \\
\text { still detailed and } \\
\text { valuable information can } \\
\text { be obtained }\end{array}$ & $\begin{array}{l}\text { + Good compatibility } \\
\text { due to small reactor } \\
\text { volumes }\end{array}$ & $\begin{array}{l}\text { + H-NMR mostly used } \\
\text { and compatible due to } \\
\text { longer scan time } \\
\text { needed for other types, } \\
\text { such as C-NMR }\end{array}$ \\
\hline
\end{tabular}

${ }^{1}$ (Typical speeds given in reviews. Please note that the speed is also dependent on the concentration that is used and the sensitivity of the machine)

${ }^{2}$ (Typical spatial resolution reported depending on the combination of chemical information needed and the catalyst material) ${ }^{156}$

\subsubsection{Conclusions}

Currently, it has been shown that many analysis techniques are available to characterize both catalyst nanoparticles and supported catalyst material insitu in a microfluidic device. For analysis of formed products at the outlet of a microreactor often GC and MS are used. The limit of these techniques however, is that no information along the length of the reactor can be obtained. Shortlived intermediates which only exist close to the catalyst cannot be detected with these techniques. In order to study catalyst performance at multiple spots in a microreactor, in-situ spectroscopic techniques such as UV-VIS, IR, RS, NMR, and XAS are used.

For nanoparticle characteristics such as size, growth, and composition XAS and UV-VIS are used, where mostly XAS is used to characterize crystal structure, oxidation state, bond distance, and coordination number.

Characterization of the activity and deactivation of supported catalyst material is mostly done in packed-bed reactors with multiple particles producing ensemble averages. The observed techniques are well suitable in combination with microfluidics. Locally formed products, differences in activity along a catalyst bed, turnover frequencies, and even the chemical state of the catalyst themselves are measurable using these techniques. 


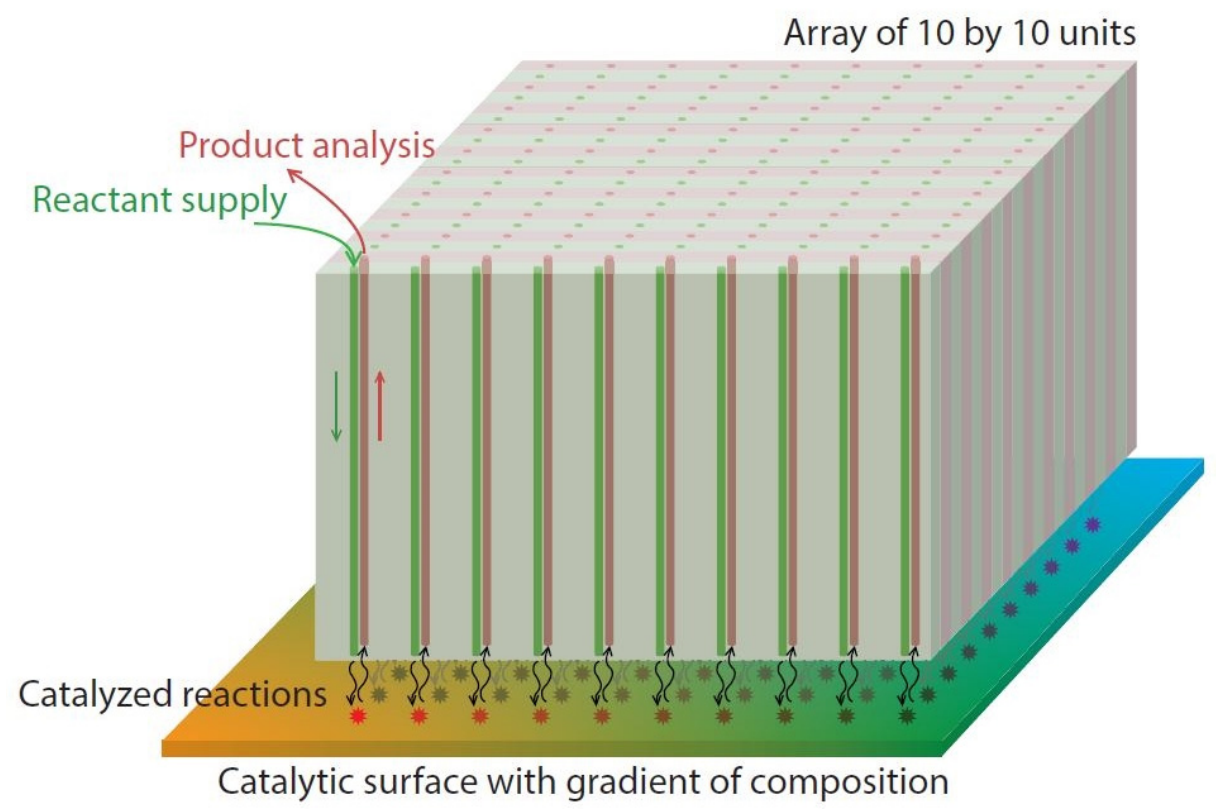

Figure 2.7. Simplified presentation of the device developed by Kondratyuk et al. ${ }^{125}$ to analyze catalytic activity simultaneously in 100 locations with high spatial resolution (100 measurement points on $\left.1 \mathrm{~cm}^{2}\right)$. Adapted from ${ }^{125}$.

\subsubsection{Perspectives on catalyst characterization in microfluidics}

Biology has found in microfluidics an efficient way to study individually cells from large populations. ${ }^{157}$ By isolating individual cells in single droplets, they can be characterized individually at high throughput, allowing the analysis of large samples without losing the information about each cell. Several reviews focus on droplet-based microfluidics ${ }^{158}$ and its applications to biology, chemistry and nanotechnology ${ }^{159}$. The need of biologists for analysis of individual elements in a large sample is very similar to the necessity for chemists to follow the evolution of individual catalyst particles. Some microfluidic tools that have been developed for biology and could be used for catalysis are presented in the next sections.

\section{Single Particle Analysis}

In biology, to analyse the chemical content of droplets containing cells, optical detection of fluorescent molecules is currently by far the mostcommonly-used method. ${ }^{160-168}$ Droplets contain a fluorescent dye that is activated by a product of the cell activity, and therefore visible only if the cell is active enough, enabling a direct readout of every cell activity. The same approach has also been used to encapsulate enzymes and to measure their 
activity in a large number of different chemical environments. ${ }^{169}$ Impedancebased measurements also showed promising results to analyse the content of droplets. ${ }^{170}$ These methods could be almost directly transposed to study the activity of individual catalyst particles by isolating them in different droplets.

\section{Single Particle Sorting}

Usually in biological analysis, the droplets and the cells that they contain are sorted in two or more categories based on the results of the aforementioned analysis. The droplets identified as containing highly active cells are sorted for further analysis. ${ }^{160-162,165,166,168}$ This could be also very useful for catalysis, for instance to select the most efficient catalysts of a population. The sorting is active, which means that fluorescent signal is captured and triggers an actuator that reroute the specific droplets in a different channel. These actuators can be based on various phenomena, but the most commonly used are electric field, ${ }^{162,168,171}$ mechanic valves, ${ }^{160,161,165,172}$ temperature, ${ }^{173,174}$ acoustic waves, ${ }^{175}$ pressure, ${ }^{166,176}$ magnetic field. ${ }^{177-179}$

\section{Particle Storage and Tagging}

Catalyst deactivation over time is a great concern for researchers and industry. The study of this phenomenon is challenging since it requires to follow the same particle over a long period of time, and this for a large number of particles to get statistically relevant data. The individual analysis of large population of particles is a challenge for which microfluidic is well adapted. However, microfluidic systems are usually characterized by short residence time, a solution must thus be found to study these long-time processes.

Very similar challenges were addressed in biology and thus, different solutions were developed. The classical method consists of isolating cells in different droplets to facilitate their manipulation, storage and analysis. Moreover, for cells as for catalyst particles, evolution can be slow and observation of significant variations can require a long time.

For very long-term processes, droplets containing the cells/particles can be extracted from the chip, incubated and injected in a new one later. ${ }^{167}$ However, when possible, in order to limit the transfer of the emulsion between chips, droplets are stored directly inside the systems. ${ }^{168}$

In some cases, it can be interesting to vary the chemical composition of the droplets to screen various incubation conditions. It is then necessary to identify the different droplets to follow the individual evolution of the cells/aging of the particles. To keep track of droplets, microscopic tags have been developed to enable later reading of their content and correlate the different measurement performed over time. ${ }^{108,163,180-182}$

It has also been shown that by confining the droplets in a line in capillaries or tubing, it is possible to keep track of the content of each droplet without tagging them and individually access them. This has been used for instance to 
study the proliferation of different cell populations over time. ${ }^{183}$ Finally, droplets can also be trapped with some obstacles inside microfluidics chips, which enables an accurate knowledge of the position of every droplet. ${ }^{165}$

It must be noted here that an important difference between cells and particles is their density: while cells have a density close to water, catalyst particles are significantly denser and therefore sediment faster, which can lead to clogging of the channels. Nevertheless, these studies of cells pave the way toward the analysis of individual catalyst particle ageing.

\section{Parallelization}

In biology as in catalysis, increasing the frequency of analysis is necessary to study larger samples. This is crucial in order to observe rare events or to find the optimal experimental conditions. This speed increase can first be achieved with pressure to increase the flow rates, and often better equipment. This can also be achieved by parallelizing the analysis process. Increasing the rate of analysis is often limited by the acquisition speed of high-speed cameras, processing time of the computers and the maximum pressure that the microfluidic chip and connectors can withstand. However, it has been shown that catalytic activity measurements could be performed locally with a high spatial resolution on a catalytic substrate of which the composition depends on position, to investigate rapidly the influence of the catalyst composition. ${ }^{125}$ Figure 2.7 shows a schematic of high-throughput catalyst screening with this method as has been shown in the characterization section.

\subsection{Perspectives for fundamental understanding}

The structure of industrial catalyst particles is complex and consists of various necessary properties such as: porosity, mechanical stability and catalytic activity of the particle. ${ }^{184}$ The mass and heat transfers inside these particles and the reaction medium are only partially understood yet. Further studies are limited by the difficulty of analysing single catalyst particles during reactions.

An in-depth understanding of the mass and heat transfers inside the particles could be reached by studying model assemblies of their main constitutive elements. A 2D model network of pores and catalysts could be incorporated inside a microfluidic chip to control the input reactants and analyse the products output. This could enable the mapping of temperature and product concentration inside the pore network. A similar approach has been used to study the phenomenon occurring when oil is pushed out a porous soil by water. A model network of pores was build inside a microfluidic chip, allowing direct observation of the phenomena occurring during oil recovery. ${ }^{185,186}$ However, in our knowledge such a method has not been transposed to the study of transport inside the pores of catalyst particles yet. 


\subsection{Outlook}

All the progress of the last decades in the field of catalyst synthesis and on-chip materials characterization brought a lot of new perspectives to obtain better control over catalyst materials synthesis and their functioning. The advantages of microfluidics in terms of chemical engineering could be used to further improve the control over the nanostructure and porosity of catalyst materials. Moreover, the tools that could enable the synthesis in even smaller droplets were presented and it was illustrated how parallelization of the devices could maintain the throughput despite the smaller size of the reactors. Based on the development of single-cell microfluidic analysis, sorting and long-term deactivation studies of individual catalysts seems within reach. Finally, a fundamental approach inspired by the work on enhanced-oil-recovery could enable an in-depth understanding of the mass and heat transfer in the reactive porous media constituting heterogeneous catalyst particles.

\subsection{Acronyms}

NPs stands for nanoparticles, NSs for Nano-Stars, NRs for Nanorods, RT for Room Temperature, NS for Not-Specified, AA for Ascorbic Acid, SC for Sodium Citrate, SA for Sodium Aluminate, PVP for poly(N-vinyl-2-pyrrolidone), THF for Tetrahydrofuran, CTABr for Cetyltrimethylammonium bromide, BMIM-Tf2N for 1-butyl-3-methylimidazolium bis(triflylmethyl-sulfonyl)imide, BMIM-BF4 for 1Butyl-3-methylimidazolium tetrafluoroborate, BMIM-BH4 for 1-butyl-3methylimidazolium tetrafluoroborate, TEOS for tetraethyl orthosilicate, CTAB for Cetrimonium bromide, DMF for N,N-dimethylformamide, SB12 for Propanesulfonate, OPD for O-phenylenediamine, MUA for 11-mercaptoundecanoic acid, PEG for Polyethylene glycol, TEG for Tryethylene glycol, EG for Ethylene glycol, PDMS for Poly- DimethylSiloxane, EFTE for Ethylene tetrafluoroethylene, EA for Ethyl Acetate, PTFE for Polytetrafluoroethylene, PFA for perfluoroalkoxyalkane, PVDF Polyvinylidene fluoride, MHA for Mercaptohexanoic acid.

\subsection{References}

1. Centi, G. \& van Santen, R. Catalysis for Renewables: From Feedstock to Enerly Production. (Wiley-VCH, Weinheim, 2007).

2. Gossett, D. R. et al. Label-free cell separation and sorting in microfluidic systems. Anal. Bioanal. Chem. 397, 3249-3267 (2010).

3. Wyatt Shields IV, C., Reyes, C. D. \& López, G. P. Microfluidic cell sorting: a review of the advances in the separation of cells from debulking to rare cell isolation. Lab Chip 15, 1230-1249 (2015).

4. Hao, N., Nie, Y. \& Zhang, J. X. J. Microfluidic synthesis of functional inorganic micro-/nanoparticles and applications in biomedical engineering. Int. Mater. Rev. 63, 461-487 (2018). 
5. Marre, S. \& Jensen, K. F. Synthesis of micro and nanostructures in microfluidic systems. Chem. Soc. Rev. 39, 1183-1202 (2010).

6. Song, Y., Hormes, J. \& Kumar, C. S. S. R. Microfluidic synthesis of nanomaterials. Small 4, 698-711 (2008).

7. Luo, G., Du, L., Wang, Y., Lu, Y. \& Xu, J. Controllable preparation of particles with microfluidics. Particuology 9, 545-558 (2011).

8. Elvira, K. S., i Solvas, X. C., Wootton, R. C. R. \& deMello, A. J. The past, present and potential for microfluidic reactor technology in chemical synthesis. Nat. Chem. 5, 905-915 (2013).

9. Duncombe, T. A., Tentori, A. M. \& Herr, A. E. Microfluidics: Reframing biological enquiry. Nat. Rev. Mol. Cell Biol. 16, 554-567 (2015).

10. Jamal, F., Jean-Sébastien, G., Maël, P., Edmond, P. \& Christian, R. Gold nanoparticle synthesis in microfluidic systems and immobilisation in microreactors designed for the catalysis of fine organic reactions. Microsyst. Technol. 18, 151-158 (2012).

11. Ftouni, J. et al. Highly controlled synthesis of nanometric gold particles by citrate reduction using the short mixing, heating and quenching times achievable in a microfluidic device. Nanoscale 4, 4450-4454 (2012).

12. Lin, S., Lin, K., Lu, D. \& Liu, Z. Journal of Environmental Chemical Engineering Preparation of uniform magnetic iron oxide nanoparticles by co-precipitation in a helical module microchannel reactor. Biochem. Pharmacol. 5, 303-309 (2017).

13. Roberts, E. J. et al. High-Throughput Continuous Flow Synthesis of Nickel Nanoparticles for the Catalytic Hydrodeoxygenation of Guaiacol. ACS Sustain. Chem. Eng. 5, 632-639 (2017).

14. Lee, P. S., Garimella, S. V. \& Liu, D. Investigation of heat transfer in rectangular microchannels. Int. J. Heat Mass Transf. 48, 1688-1704 (2005).

15. Morini, G. L. Single-phase convective heat transfer in microchannels: A review of experimental results. Int. J. Therm. Sci. 43, 631-651 (2004).

16. Rosa, P., Karayiannis, T. G. \& Collins, M. W. Single-phase heat transfer in microchannels: The importance of scaling effects. Appl. Therm. Eng. 29, 3447-3468 (2009).

17. Palm, B. Heat transfer in rectangular microchannels. Microscale Thermophys. Eng. 5, 155-175 (2001).

18. López-Lorente, Á. I., Valcárcel, M. \& Mizaikoff, B. Continuous flow synthesis and characterization of tailor-made bare gold nanoparticles for use in SERS. Microchim. Acta 181, 1101-1108 (2014).

19. SadAbadi, H., Badilescu, S., Packirisamy, M. \& Wüthrich, R. Integration of gold nanoparticles in PDMS microfluidics for lab-on-a-chip plasmonic biosensing of growth hormones. Biosens. Bioelectron. 44, 77-84 (2013).

20. Park, J. Il, Saffari, A., Kumar, S., Günther, A. \& Kumacheva, E. Microfluidic Synthesis of Polymer and Inorganic Particulate Materials. Annu. Rev. Mater. Res. 40, 415-443 (2010). 
21. Tofighi, G. et al. Continuous microfluidic synthesis of colloidal ultrasmall gold nanoparticles: in situ study of the early reaction stages and application for catalysis. React. Chem. Eng. 2, 876-884 (2017).

22. Wang, L., Ma, S., Yang, B., Cao, W. \& Han, X. Morphology-controlled synthesis of Ag nanoparticle decorated poly(o-phenylenediamine) using microfluidics and its application for hydrogen peroxide detection. Chem. Eng. J. 268, 102-108 (2015).

23. Jun, H. et al. Understanding of the size control of biocompatible gold nanoparticles in millifluidic channels. Langmuir 28, 15966-15974 (2012).

24. Polte, J. et al. Nucleation and Growth of Gold. ACS Nano 4, 1076-1082 (2010).

25. Xu, L. et al. Microfluidic reactor synthesis and photocatalytic behavior of Cu@Cu2O nanocomposite. Appl. Surf. Sci. 331, 449-454 (2015).

26. Xu, L., Srinivasakannan, C., Peng, J., Zhang, L. \& Zhang, D. Synthesis of $\mathrm{Cu}-\mathrm{CuO}$ nanocomposite in microreactor and its application to photocatalytic degradation. J. Alloys Compd. 695, 263-269 (2017).

27. Hayashi, N., Sakai, Y., Tsunoyama, H. \& Nakajima, A. Development of ultrafine multichannel microfluidic mixer for synthesis of bimetallic nanoclusters: Catalytic application of highly monodisperse $\mathrm{AuPd}$ nanoclusters stabilized by poly(N -vinylpyrrolidone). Langmuir 30, 10539-10547 (2014).

28. Baber, R., Mazzei, L., Thanh, N. T. K. \& Gavriilidis, A. Synthesis of silver nanoparticles in a microfluidic coaxial flow reactor. RSC Adv. 5, 9558595591 (2015).

29. Abou-Hassan, A., Neveu, S., Dupuis, V. \& Cabuil, V. Synthesis of cobalt ferrite nanoparticles in continuous-flow microreactors. RSC Adv. 2, 11263-11266 (2012).

30. Yagyu, H., Tanabe, Y., Takano, S. \& Hamamoto, M. Continuous flow synthesis of monodisperse gold nanoparticles by liquid-phase reduction method on glass microfluidic device. Micro Nano Lett. 12, 536-539 (2017).

31. Tofighi, G. et al. Continuous microfluidic synthesis of colloidal ultrasmall gold nanoparticles: in situ study of the early reaction stages and application for catalysis. React. Chem. Eng. 2, 876-884 (2017).

32. Bandulasena, M. V., Vladisavljević, G. T., Odunmbaku, O. G. \& Benyahia, B. Continuous synthesis of PVP stabilized biocompatible gold nanoparticles with a controlled size using a 3D glass capillary microfluidic device. Chem. Eng. Sci. 171, 233-243 (2017).

33. Uson, L., Sebastian, V., Arruebo, M. \& Santamaria, J. Continuous microfluidic synthesis and functionalization of gold nanorods. Chem. Eng. J. 285, 286-292 (2016).

34. Fu, Q. et al. Growth Mechanism Deconvolution of Self- Growth Mechanism Deconvolution of Self-Limiting Supraparticles Based on Micro fl uidic System. ACS Nano 9, 172-179 (2015). 
35. Gomez, L. et al. Scaled-up production of plasmonic nanoparticles using microfluidics: from metal precursors to functionalized and sterilized nanoparticles. Lab Chip 14, 325-332 (2014).

36. Lohse, S. E., Eller, J. R., Sivapalan, S. T., Plews, M. R. \& Murphy, C. J. A simple millifluidic benchtop reactor system for the high-throughput synthesis and functionalization of gold nanoparticles with different sizes and shapes. ACS Nano 7, 4135-4150 (2013).

37. Sai Krishna, K. et al. Millifluidics for time-resolved mapping of the growth of gold nanostructures. J. Am. Chem. Soc. 135, 5450-5456 (2013).

38. Sugie, A. et al. Synthesis of thiol-capped gold nanoparticle with a flow system using organosilane as a reducing agent. Tetrahedron Lett. 53, 44574459 (2012).

39. Han, K. N. et al. On-chip electrochemical detection of bio/chemical molecule by nanostructures fabricated in a microfluidic channel. Sensors Actuators, B Chem. 177, 472-477 (2013).

40. Kitson, P. J., Rosnes, M. H., Sans, V., Dragone, V. \& Cronin, L. Configurable 3D-Printed millifluidic and microfluidic 'lab on a chip' reactionware devices. Lab Chip 12, 3267-3271 (2012).

41. Luty-Błocho, M. et al. Synthesis of gold nanoparticles in an interdigital micromixer using ascorbic acid and sodium borohydride as reducers. Chem. Eng. J. 171, 279-290 (2011).

42. Ishizaka, T., Ishigaki, A., Kawanami, H., Suzuki, A. \& Suzuki, T. M. Dynamic control of gold nanoparticle morphology in a microchannel flow reactor by glucose reduction in aqueous sodium hydroxide solution. J. Colloid Interface Sci. 367, 135-138 (2012).

43. Sugano, K. et al. Mixing speed-controlled gold nanoparticle synthesis with pulsed mixing microfluidic system. Microfluid. Nanofluidics 9, 11651174 (2010).

44. Pedro, S. G. et al. Synthesis of MUA-protected gold nanoparticles in microfluidic devices with in situ UV-vis characterization. Conf. Ibersensor November, Lisbon (2010).

45. Bullen, C., Latter, M. J., D'Alonzo, N. J., Willis, G. J. \& Raston, C. L. A seedless approach to continuous flow synthesis of gold nanorods. Chem. Commun. 47, 4123-4125 (2011).

46. Horikoshi, S., Sumi, T. \& Serpone, N. A Hybrid Microreactor/Microwave High-Pressure Flow System of a Novel Concept Design and its Application to the Synthesis of Silver Nanoparticles. Chem. Eng. Process. Process Intensif. 73, 59-66 (2013).

47. Liu, H. et al. Microfluidic biosynthesis of silver nanoparticles: Effect of process parameters on size distribution. Chem. Eng. J. 209, 568-576 (2012).

48. Karim, A. M. et al. Synthesis of $1 \mathrm{~nm}$ Pd Nanoparticles in a Microfluidic Reactor: Insights from in Situ X-ray Absorption Fine Structure Spectroscopy and Small-Angle X-ray Scattering. J. Phys. Chem. C. 119, 
13257-13267 (2015).

49. Liang, H. et al. Synthesis of Fe(1-x)Znx@Zn(1-y)FeyOz nanocrystals via a simple programmed microfluidic process. Mater. Chem. Phys. 201, 156164 (2017).

50. Jiao, M., Zeng, J., Jing, L., Liu, C. \& Gao, M. Flow synthesis of biocompatible $\mathrm{Fe} 3 \mathrm{O} 4$ nanoparticles: Insight into the effects of residence time, fluid velocity, and tube reactor dimension on particle size distribution. Chem. Mater. 27, 1299-1305 (2015).

51. Song, Y., Li, R., Sun, Q. \& Jin, P. Controlled growth of Cu nanoparticles by a tubular microfluidic reactor. Chem. Eng. J. 168, 477-484 (2011).

52. Xu, L., Srinivasakannan, C., Peng, J., Zhang, D. \& Chen, G. Synthesis of nickel nanoparticles by aqueous reduction in continuous flow microreactor. Chem. Eng. Process. Process Intensif. 93, 44-49 (2015).

53. Eluri, R. \& Paul, B. Synthesis of nickel nanoparticles by hydrazine reduction: Mechanistic study and continuous flow synthesis. J. Nanoparticle Res. 14, 800, (2012).

54. Hafermann, L. \& Köhler, J. M. Photochemical Micro Continuous-Flow Synthesis of Noble Metal Nanoparticles of the Platinum Group. Chem. Eng. Technol. 38, 1138-1143 (2015).

55. Suryawanshi, P. L., Gumfekar, S. P., Kumar, P. R., Kale, B. B. \& Sonawane, S. H. Synthesis of ultra-small platinum nanoparticles in a continuous flow microreactor. Colloids Interface Sci. Commun. 13, 6-9 (2016).

56. Hossain, M. J., Tsunoyama, H., Yamauchi, M., Ichikuni, N. \& Tsukuda, T. High-yield synthesis of PVP-stabilized small Pt clusters by microfluidic method. Catal. Today 183, 101-107 (2012).

57. Abalde-Cela, S., Taladriz-Blanco, P., De Oliveira, M. G. \& Abell, C. Droplet microfluidics for the highly controlled synthesis of branched gold nanoparticles. Sci. Rep. 8, 2440-2446 (2018).

58. Lazarus, L. L. et al. Two-phase microfluidic droplet flows of ionic liquids for the synthesis of gold and silver nanoparticles. ACS Appl. Mater. Interfaces 4, 3077-3083 (2012).

59. Lazarus, L. L., Yang, A. S.-J., Chu, S., Brutchey, R. L. \& Malmstadt, N. Flow-focused synthesis of monodisperse gold nanoparticles using ionic liquids on a microfluidic platform. Lab Chip 10, 3377-3379 (2010).

60. Xu, L. et al. Synthesis of copper nanoparticles by a T-shaped microfluidic device. RSC Adv. 4, 25155-25159 (2014).

61. Zhang, L., Wang, Y., Tong, L. \& Xia, Y. Seed-mediated synthesis of silver nanocrystals with controlled sizes and shapes in droplet microreactors separated by air. Langmuir 29, 15719-15725 (2013).

62. Hafermann, L. \& Michael Köhler, J. Small gold nanoparticles formed by rapid photochemical flow-through synthesis using microfluid segment technique. J. Nanoparticle Res. 17, 1-8 (2015). 
63. Kim, Y. H. et al. Droplet-based microreactors for continuous production of palladium nanocrystals with controlled sizes and shapes. Small 9, 3462-3467 (2013).

64. Kumar, K. et al. Direct synthesis of dextran-coated superparamagnetic iron oxide nanoparticles in a capillary-based droplet reactor. J. Mater. Chem. 22, 4704-4708 (2012).

65. Lee, K. G. et al. In VitroBiosynthesis of Metal Nanoparticles in Microdroplets. ACS Nano 6, 6998-7008 (2012).

66. Zhang, L. et al. Continuous and scalable production of well-controlled noble-metal nanocrystals in milliliter-sized droplet reactors. Nano Lett. 14, 6626-6631 (2014).

67. Li, X. et al. Hierarchically structured particles for micro flow catalysis. Chem. Eng. J. 326, 1058-1065 (2017).

68. Ravi Kumar, D. V., Prasad, B. L. V. \& Kulkarni, A. A. Segmented flow synthesis of Ag nanoparticles in spiral microreactor: Role of continuous and dispersed phase. Chem. Eng. J. 192, 357-368 (2012).

69. Sebastian Cabeza, V., Kuhn, S., Kulkarni, A. A. \& Jensen, K. F. SizeControlled Flow Synthesis of Gold Nanoparticles Using a Segmented Flow Micro fluidic Platform. Langmuir 28, 7007-7013 (2012).

70. Zhang, D. et al. One-step, facile and ultrafast synthesis of phase- and sizecontrolled Pt-Bi intermetallic nanocatalysts through continuous-flow microfluidics. J. Am. Chem. Soc. 137, 6263-6269 (2015).

71. Wang, Z. et al. Microfluidic Synthesis and Characterization of FePtSn/C Catalysts with Enhanced Electro-Catalytic Performance for Direct Methanol Fuel Cells. Electrochim. Acta 230, 245-254 (2017).

72. Tao, S., Yang, M., Chen, H., Ren, M. \& Chen, G. Microfluidic synthesis of Ag@Cu2O core-shell nanoparticles with enhanced photocatalytic activity. J. Colloid Interface Sci. 486, 16-26 (2017).

73. Tian, Z. H., Wang, Y. J., Xu, J. H. \& Luo, G. S. Intensification of nucleation stage for synthesizing high quality CdSe quantum dots by using preheated precursors in microfluidic devices. Chem. Eng. J. 302, 498-502 (2016).

74. Tian, Z. H., Xu, J. H., Wang, Y. J. \& Luo, G. S. Microfluidic synthesis of monodispersed CdSe quantum dots nanocrystals by using mixed fatty amines as ligands. Chem. Eng. J. 285, 20-26 (2016).

75. Swain, B. et al. Optimization of CdSe nanocrystals synthesis with a microfluidic reactor and development of combinatorial synthesis process for industrial production. Chem. Eng. J. 308, 311-321 (2017).

76. Wang, J., Zhao, H., Zhu, Y. \& Song, Y. Shape-Controlled Synthesis of CdSe Nanocrystals via a Programmed Microfluidic Process. J. Phys. Chem. C 121, 3567-3572 (2017).

77. Lignos, I., Stavrakis, S., Kilaj, A. \& deMello, A. J. Millisecond-Timescale Monitoring of PbS Nanoparticle Nucleation and Growth Using Droplet- 
Based Microfluidics. Small 11, 4009-4017 (2015).

78. Carroll, N. J. et al. Microfluidic synthesis of monodisperse nanoporous oxide particles and control of hierarchical pore structure. ACS Appl. Mater. Interfaces 5, 3524-3529 (2013).

79. Bchellaoui, N., Hayat, Z., Mami, M., Dorbez-Sridi, R. \& El Abed, A. I. Microfluidic-assisted Formation of Highly Monodisperse and Mesoporous Silica Soft Microcapsules. Sci. Rep. 7, 16326, (2017).

80. Nie, Y., Hao, N. \& Zhang, J. X. J. Ultrafast Synthesis of Multifunctional Submicrometer Hollow Silica Spheres in Microfluidic Spiral Channels. Sci. Rep. 7, 12616, (2017).

81. Lee, I., Yoo, Y., Cheng, Z. \& Jeong, H. K. Generation of monodisperse mesoporous silica microspheres with controllable size and surface morphology in a microfluidic device. Adv. Funct. Mater. 18, 4014-4021 (2008).

82. Yu, L., Pan, Y., Wang, C. \& Zhang, L. A two-phase segmented microfluidic technique for one-step continuous versatile preparation of zeolites. Chem. Eng. J. 219, 78-85 (2013).

83. Ju, J., Zeng, C., Zhang, L. \& Xu, N. Continuous synthesis of zeolite NaA in a microchannel reactor. Chem. Eng. J. 116, 115-121 (2006).

84. Pan, Y., Yao, J., Zhang, L. \& Xu, N. Preparation of Ultrafine Zeolite A Crystals with Narrow Particle Size Distribution Using a Two-Phase Liquid Segmented Microfluidic Reactor. Ind. Eng. Chem. Res. 48, 84718477 (2009).

85. Hoang, P. H., Yoon, K. B. \& Kim, D. P. Synthesis of hierarchically porous zeolite A crystals with uniform particle size in a droplet microreactor. RSC Adv. 2, 5323-5328 (2012).

86. Tice, J. D., Song, H., Lyon, A. D. \& Ismagilov, R. F. Formation of Droplets and Mixing in Multiphase Microfluidics at Low Values of the Reynolds and the Capillary Numbers. Langmuir 19, 9127-9133 (2003).

87. Grigoriev, R. O., Schatz, M. F. \& Sharma, V. Chaotic mixing in microdroplets. Lab Chip 6, 1369-1372, (2006).

88. Nightingale, A. M. \& De Mello, J. C. Microscale synthesis of quantum dots. J. Mater. Chem. 20, 8454-8463 (2010).

89. Baek, J., Shen, Y., Lignos, I., Bawendi, M. G. \& Jensen, K. F. Multistage Microfluidic Platform for the Continuous Synthesis of III-V Core/Shell Quantum Dots. Angew. Chemie - Int. Ed. 57, 10915-10918 (2018).

90. Rimer, J. D., Kumar, M., Li, R., Lupulescu, A. I. \& Oleksiak, M. D. Tailoring the physicochemical properties of zeolite catalysts. Catal. Sci. Technol. 4, 3762-3771 (2014).

91. Pan, Y. et al. Rapid crystallization of silicalite nanocrystals in a capillary microreactor. Chem. Eng. Technol. 32, 732-737 (2009).

92. Liu, Y. \& Jiang, X. Why microfluidics? Merits and trends in chemical synthesis. Lab Chip 17, 3960-3978 (2017). 
93. Malloggi, F. et al. Monodisperse colloids synthesized with nanofluidic technology. Langmuir 26, 2369-2373 (2010).

94. Amstad, E. et al. Parallelization of microfluidic flow-focusing devices. Phys. Rev. E 95, 43105, (2017).

95. Leman, M., Abouakil, F., Griffiths, A. D. \& Tabeling, P. Droplet-based microfluidics at the femtolitre scale. Lab Chip 15, 753-765 (2015).

96. Toshima, N. \& Yonezawa, T. Bimetallic nanoparticles-novel materials for chemical and physical applications. New J. Chem. 22, 1179-1201 (1998).

97. Ferrer, D. et al. Three-layer core/shell structure in Au-Pd bimetallic nanoparticles. Nano Lett. 7, 1701-1705 (2007).

98. Park, J. H., Han, C. M., Lee, E. J. \& Kim, H. W. Preparation of highly monodispersed porous-channeled poly(caprolactone) microspheres by a microfluidic system. Mater. Lett. 181, 92-98 (2016).

99. Sung, I. K., Christian, Mitchell, M., Kim, D. P. \& Kenis, P. J. A. Tailored macroporous $\mathrm{SiCN}$ and $\mathrm{SiC}$ structures for high-temperature fuel reforming. Adv. Funct. Mater. 15, 1336-1342 (2005).

100. De Nijs, B. et al. Entropy-driven formation of large icosahedral colloidal clusters by spherical confinement. Nat. Mater. 14, 56-60 (2015).

101. Lee, M. N. \& Mohraz, A. Bicontinuous macroporous materials from bijel templates. Adv. Mater. 22, 4836-4841 (2010).

102. Haase, M. F., Stebe, K. J. \& Lee, D. Continuous Fabrication of Hierarchical and Asymmetric Bijel Microparticles, Fibers, and Membranes by Solvent Transfer-Induced Phase Separation (STRIPS). Adv. Mater. 27, 7065-7071 (2015).

103. Amstad, E., Spaepen, F., Brenner, M. P. \& Weitz, D. A. The microfluidic nebulator: production of sub-micrometer sized airborne drops. Lab Chip 17, 1475-1480 (2017).

104. Yadavali, S., Jeong, H. H., Lee, D. \& Issadore, D. Silicon and glass very large scale microfluidic droplet integration for terascale generation of polymer microparticles. Nat. Commun. 9, 1222 (2018).

105. Amstad, E. et al. Robust scalable high throughput production of monodisperse drops. Lab Chip 16, 4163-4172 (2016).

106. Ofner, A. et al. High-Throughput Step Emulsification for the Production of Functional Materials Using a Glass Microfluidic Device. Macromol. Chem. Phys. 218, 1600472, 1-10 (2017).

107. $\mathrm{Xu}, \mathrm{X}$. et al. High aspect ratio induced spontaneous generation of monodisperse picolitre droplets for digital PCR. Biomicrofluidics 12, 14103 (2018).

108. Zhao, S. et al. Operando Characterization of Catalysts through use of a Portable Microreactor. ChemCatChem 7, 3683-3691 (2015).

109. Zhang, Q., Zhang, Q., Wang, H. \& Li, Y. A high efficiency microreactor with $\mathrm{Pt} / \mathrm{ZnO}$ nanorod arrays on the inner wall for photodegradation of 
phenol. J. Hazard. Mater. 254-255, 318-324 (2013).

110. He, Z., Li, Y., Zhang, Q. \& Wang, H. Capillary microchannel-based microreactors with highly durable $\mathrm{ZnO} / \mathrm{TiO} 2$ nanorod arrays for rapid, high efficiency and continuous-flow photocatalysis. Appl. Catal. B Environ. 93, 376-382 (2010).

111. Hannemann, S. et al. Distinct spatial changes of the catalyst structure inside a fixed-bed microreactor during the partial oxidation of methane over Rh/Al2O3. Catal. Today 126, 54-63 (2007).

112. Bouchard, L.-S. et al. NMR Imaging of Catalytic Hydrogenation in Microreactors with the Use of para-Hydrogen. Science (80-. ). 319, 442445 (2008).

113. Zhang, H., Wang, J. J., Fan, J. \& Fang, Q. Microfluidic chip-based analytical system for rapid screening of photocatalysts. Talanta 116, 946950 (2013).

114. Liu, A. L., Li, Z. Q., Wu, Z. Q. \& Xia, X. H. Study on the photocatalytic reaction kinetics in a $\mathrm{TiO} 2$ nanoparticles coated microreactor integrated microfluidics device. Talanta 182, 544-548 (2018).

115. Gmeiner, J., Behrens, S., Spliethoff, B. \& Trapp, O. Ruthenium Nanoparticles in High-Throughput Studies of Chemoselective Carbonyl Hydrogenation Reactions. ChemCatChem 8, 571-576 (2016).

116. Gross, E. et al. In situ IR and X-ray high spatial-resolution microspectroscopy measurements of multistep organic transformation in flow microreactor catalyzed by Au nanoclusters. J. Am. Chem. Soc. 136, 3624-3629 (2014).

117. Zhang, Z. et al. Catalysis by Metal Nanoparticles in a Plug-In Optofluidic Platform: Redox Reactions of p-Nitrobenzenethiol and $\mathrm{p}$ Aminothiophenol. ACS Catal. 8, 2443-2449 (2018).

118. Lin, X. Z., Terepka, A. D. \& Yang, H. Synthesis of silver nanoparticles in a continuous flow tubular microreactor. Nano Lett. 4, 2227-2232 (2004).

119. Biswas, S., Miller, J. T., Li, Y., Nandakumar, K. \& Kumar, C. S. S. R. Developing a millifluidic platform for the synthesis of ultrasmall nanoclusters: Ultrasmall copper nanoclusters as a case study. Small 8, 688-698 (2012).

120. Navin, C. V. et al. Investigation of the synthesis and characterization of platinum-DMSA nanoparticles using millifluidic chip reactor. Chem. Eng. J. 281, 81-86 (2015).

121. Sankar, G., Cao, E. \& Gavriilidis, A. A microstructured reactor based in situ cell for the study of catalysts by X-ray absorption spectroscopy under operating conditions. Catal. Today 125, 24-28 (2007).

122. Pattekar, A. V. \& Kothare, M. V. A microreactor for hydrogen production in micro fuel cell applications. J. Microelectromechanical Syst. 13, 7-18 (2004).

123. Li, H., Yu, X., Tu, S. T., Yan, J. \& Wang, Z. Catalytic performance and 
characterization of Al2O 3-supported Pt-Co catalyst coatings for preferential CO oxidation in a micro-reactor. Appl. Catal. A Gen. 387, 215223 (2010).

124. Henriksen, T. R., Olsen, J. L., Vesborg, P., Chorkendorff, I. \& Hansen, O. Highly sensitive silicon microreactor for catalyst testing. Rev. Sci. Instrum. 80, 124101 (2009).

125. Kondratyuk, P. et al. A microreactor array for spatially resolved measurement of catalytic activity for high-throughput catalysis science. J. Catal. 300, 55-62 (2013).

126. Ajmera, S. K., Delattre, C., Schmidt, M. A. \& Jensen, K. F. Microfabricated differential reactor for heterogeneous gas phase catalyst testing. J. Catal. 209, 401-412 (2002).

127. Ajmera, S. K., Delattre, C., Schmidt, M. A. \& Jensen, K. F. Microreactors for measuring catalyst activity and determining reaction kinetics. Sci. Technol. Catal. 2002 145, 97-102 (2003).

128. Hannemann, S. et al. Axial changes of catalyst structure and temperature in a fixed-bed microreactor during noble metal catalysed partial oxidation of methane. Top. Catal. 52, 1360-1370 (2009).

129. Cao, E. et al. Reaction and Raman spectroscopic studies of alcohol oxidation on gold-palladium catalysts in microstructured reactors. Chem. Eng. J. 167, 734-743 (2011).

130. Zhivonitko, V. V., Telkki, V. V. \& Koptyug, I. V. Characterization of microfluidic gas reactors using remote-detection MRI and parahydrogen-induced polarization. Angew. Chemie - Int. Ed. 51, 80548058 (2012).

131. Olivon, K. \& Sarrazin, F. Heterogeneous reaction with solid catalyst in droplet-flow millifluidic device. Chem. Eng. J. 227, 97-102 (2013).

132. Ponce, S. et al. Stable Immobilization of Size-Controlled Bimetallic Nanoparticles in Photonic Crystal Fiber Microreactor. Chemie Ing. Tech. 90, 653-659 (2018).

133. Aguirre, A., Kler, P. A., Berli, C. L. A. \& Collins, S. E. Design and operational limits of an ATR-FTIR spectroscopic microreactor for investigating reactions at liquid-solid interface. Chem. Eng. J. 243, 197206 (2014).

134. Xie, W., Grzeschik, R. \& Schlücker, S. Metal Nanoparticle-Catalyzed Reduction Using Borohydride in Aqueous Media: A Kinetic Analysis of the Surface Reaction by Microfluidic SERS. Angew. Chemie - Int. Ed. 55, 13729-13733 (2016).

135. Ricciardi, R., Huskens, J. \& Verboom, W. Influence of the Au/Ag ratio on the catalytic activity of dendrimer-encapsulated bimetallic nanoparticles in microreactors. J. Flow Chem. 5, 228-233 (2015).

136. Li, Y. et al. Real-time spectroscopic monitoring of photocatalytic activity promoted by graphene in a microfluidic reactor. Sci. Rep. 6, 28803 (2016). 
137. mempax@www.pgo-online.com. Available at: https://www.pgo online.com/intl/ mempax.html.

138. Song, Y. et al. Microfluidic synthesis of cobalt nanoparticles. Chem. Mater. 18, 2817-2827 (2006).

139. Bartholomew, C. H. Mechanism of catalyst deactivation. Appl. Catal. A Gen. 212, 17-60 (2001).

140. Karabudak, E. Micromachined silicon attenuated total reflectance infrared spectroscopy: An emerging detection method in micro/nanofluidics. Electrophoresis 35, 236-244 (2014).

141. Meirer, F. et al. Agglutination of single catalyst particles during fluid catalytic cracking as observed by X-ray nanotomography. Chem. Commun. 51, 8097-8100 (2015).

142. Jin, M., Pully, V., Otto, C., Van Den Berg, A. \& Carlen, E. T. High-density periodic arrays of self-aligned subwavelength nanopyramids for surface-enhanced Raman spectroscopy. J. Phys. Chem. C 114, 21953-21959 (2010).

143. Baertsch, C. D., Schmidt, M. A. \& Jensen, K. F. Catalyst surface characterization in microfabricated reactors using pulse chemisorption. Chem Commun 2610-2611 (2004).

144. Lee, S. K., Liu, X., Sebastián Cabeza, V. \& Jensen, K. F. Synthesis, assembly and reaction of a nanocatalyst in microfluidic systems: a general platform. Lab Chip 12, 4080-4084 (2012).

145. Tiggelaar, R. M. et al. Fabrication, mechanical testing and application of high-pressure glass microreactor chips. Chem. Eng. J. 131, 163-170 (2007).

146. Tiggelaar, R. M. et al. Fabrication of a high-temperature microreactor with integrated heater and sensor patterns on an ultrathin silicon membrane. Sensors Actuators, A Phys. 119, 196-205 (2005).

147. Buurmans, I. L. C. et al. Catalytic activity in individual cracking catalyst particles imaged throughout different life stages by selective staining. Nat. Chem. 3, 862-867 (2011).

148. Morra, G. et al. High-throughput gas phase transient reactor for catalytic material characterization and kinetic studies. Chem. Eng. J. 138, 379-388 (2008).

149. Childs, D. T. D. et al. Sensitivity Advantage of QCL Tunable-Laser MidInfrared Spectroscopy over FTIR Spectroscopy. Appl. Spectrosc. Rev. 50, 822-839 (2015).

150. Schlindwein, W. et al. In-line uv-vis spectroscopy as a fast-working process analytical technology (Pat) during early phase product development using hot melt extrusion (hme). Pharmaceutics 10, 1.-25 (2018).

151. Kumamoto, Y., Harada, Y., Takamatsu, T. \& Tanaka, H. Label-free Molecular Imaging and Analysis by Raman Spectroscopy. Acta Histochem. Cytochem. 51, 101-110 (2018). 
152. Real-Time Gas Analysis and Reaction Monitoring using Mass Spectrometry. Available at: https://www.hidenanalytical.com/real-timegas-analysis-reaction-monitoring-using-mass-spectrometry/.

153. Seres, E. \& Spielmann, C. Ultrafast soft x-ray absorption spectroscopy with sub- 20-fs resolution. Appl. Phys. Lett. 91, 89-92 (2007).

154. Müller, O., Nachtegaal, M., Just, J., Lützenkirchen-Hecht, D. \& Frahm, R. Quick-EXAFS setup at the SuperXAS beamline for in situ X-ray absorption spectroscopy with 10ms time resolution. J. Synchrotron Radiat. 23, 260-266 (2016).

155. Palmer, A. G. NMR Characterization of the Dynamics of Biomacromolecules. Chem. Rev. 104, 3623-3640 (2004).

156. Buurmans, I. L. C. \& Weckhuysen, B. M. Heterogeneities of individual catalyst particles in space and time as monitored by spectroscopy. Nat. Chem. 4, 873-886 (2012).

157. Yin, H. \& Marshall, D. Microfluidics for single cell analysis. Curr. Opin. Biotechnol. 23, 110-119 (2012).

158. Seemann, R., Brinkmann, M., Pfohl, T. \& Herminghaus, S. Droplet based microfluidics. Reports Prog. Phys. 75, 16601 (2012).

159. Mashaghi, S., Abbaspourrad, A., Weitz, D. A. \& van Oijen, A. M. Droplet microfluidics: A tool for biology, chemistry and nanotechnology. Trends Anal. Chem. 82, 118-125 (2016).

160. Krüger, J. et al. Development of a microfluidic device for fluorescence activated cell sorting. J. Micromechanics Microengineering 12, 486-494 (2002).

161. Studer, V., Jameson, R., Pellereau, E., Pépin, A. \& Chen, Y. A microfluidic mammalian cell sorter based on fluorescence detection. Microelectron. Eng. 73-74, 852-857 (2004).

162. Baret, J. C. et al. Fluorescence-activated droplet sorting (FADS): Efficient microfluidic cell sorting based on enzymatic activity. Lab Chip 9, 18501858 (2009).

163. Brouzes, E. et al. Droplet microfluidic technology for single-cell highthroughput screening. Proc. Natl. Acad. Sci. 106, 14195-14200 (2009).

164. Mazutis, L., Baret, J. C. \& Griffiths, A. D. A fast and efficient microfluidic system for highly selective one-to-one droplet fusion. Lab Chip 9, 26652672 (2009).

165. Abate, A. R., Agresti, J. J. \& Weitz, D. A. Microfluidic sorting with highspeed single-layer membrane valves. Appl. Phys. Lett. 96, 203509 (2010).

166. Wu, T. H. et al. Pulsed laser triggered high speed microfluidic fluorescence activated cell sorter. Lab Chip 12, 1378-1383 (2012).

167. Mazutis, L. et al. Single-cell analysis and sorting using droplet-based microfluidics. Nat. Protoc. 8, 870-891 (2013).

168. Hammar, P. et al. Single-cell screening of photosynthetic growth and lactate production by cyanobacteria. Biotechnol. Biofuels 8, 193 (2015). 
169. Sjostrom, S. L., Joensson, H. N. \& Svahn, H. A. Multiplex analysis of enzyme kinetics and inhibition by droplet microfluidics using picoinjectors. Lab Chip 13, 1754-1761 (2013).

170. Kemna, E. W. M., Segerink, L. I., Wolbers, F., Vermes, I. \& Van Den Berg, A. Label-free, high-throughput, electrical detection of cells in droplets. Analyst 138, 4585-4592 (2013).

171. Ahn, K. et al. Dielectrophoretic manipulation of drops for high-speed microfluidic sorting devices. Appl. Phys. Lett. 88, 024104 (2006).

172. Lai, C. W., Lin, Y. H. \& Lee, G. Bin. A microfluidic chip for formation and collection of emulsion droplets utilizing active pneumatic microchoppers and micro-switches. Biomed. Microdevices 10, 749-756 (2008).

173. Baroud, C. N., Delville, J. P., Gallaire, F. \& Wunenburger, R. Thermocapillary valve for droplet production and sorting. Phys. Rev. E Stat. Nonlinear, Soft Matter Phys. 75, 046302 (2007).

174. Cordero, M. L., Burnham, D. R., Baroud, C. N. \& McGloin, D. Thermocapillary manipulation of droplets using holographic beam shaping: Microfluidic pin ball. Appl. Phys. Lett. 93, 2006-2009 (2008).

175. Franke, T., Abate, A. R., Weitz, D. A. \& Wixforth, A. Surface acoustic wave (SAW) directed droplet flow in microfluidics for PDMS devices. Lab Chip 9, 2625-2627 (2009).

176. Chen, P. et al. Hydrodynamic gating valve for microfluidic fluorescenceactivated cell sorting. Anal. Chim. Acta 663, 1-6 (2010).

177. Zhang, K. et al. On-chip manipulation of continuous picoliter-volume superparamagnetic droplets using a magnetic force. Lab Chip 9, 29922999 (2009).

178. Surenjav, E., Priest, C., Herminghaus, S. \& Seemann, R. Manipulation of gel emulsions by variable microchannel geometry. Lab Chip 9, 325-330 (2009).

179. Solsona, M. et al. Magnetophoretic Sorting of Single Catalyst Particles. Angew. Chemie - Int. Ed. 57, 10589-10594 (2018).

180. Dejneka, M. J. et al. Rare earth-doped glass microbarcodes. Proc. Natl. Acad. Sci. 100, 389-393 (2003).

181. Fang, Y., Yu, N., Jiang, Y. \& Dang, C. High-precision lens-less flow cytometer on a chip. Micromachines 9, 227 (2018).

182. Zhao, Y. et al. Microfluidic generation of multifunctional quantum dot barcode particles. J. Am. Chem. Soc. 133, 8790-8793 (2011).

183. Damodaran, S. P. et al. A millifluidic study of cell-to-cell heterogeneity in growth-rate and cell-division capability in populations of isogenic cells of Chlamydomonas reinhardtii. PLoS One 10, e0118987 (2015).

184. Vogt, E. T. C. \& Weckhuysen, B. M. Fluid catalytic cracking: recent developments on the grand old lady of zeolite catalysis. Chem. Soc. Rev. 44, 7342-7370 (2015).

185. Cottin, C., Bodiguel, H. \& Colin, A. Influence of wetting conditions on 
drainage in porous media: A microfluidic study. Phys. Rev. E - Stat. Nonlinear, Soft Matter Phys. 84, 026311 (2011).

186. Odier, C., Levaché, B., Santanach-Carreras, E. \& Bartolo, D. Forced Imbibition in Porous Media: A Fourfold Scenario. Phys. Rev. Lett. 119, 208005 (2017). 



\section{Chapter

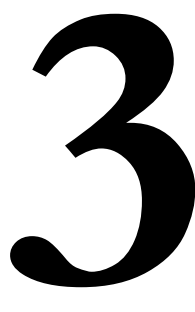

\section{Magnetophoretic Sorting of Single Catalyst Particles}

better understanding of the deactivation processes taking place within solid

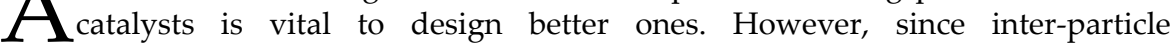
heterogeneities are more rule than exception, particle sorting is crucial to analyse catalyst particles in an effective manner. Microfluidics offers new possibilities to sort solid catalysts at the single particle level and in a high-throughput fashion. In this chapter, we report a first-of-its-kind 3-D printed magnetophoretic chip able to sort catalyst particles by their magnetic moment. Fluid Catalytic Cracking (FCC) particles were separated based on their Fe content, one of the deactivating metals. With the magnetophoretic sorting, we show that among the $20 \%$ of FCC catalyst particles with the highest Fe content, many particles have large Fe aggregates. Magnetic properties point into the direction that differences in magnetic moment are caused by an increased number and similar size of Fe containing nano-clusters. Availability of acid sites generally decreases with increasing Fe content, however, we found large acidity differences among the catalyst particles of each fraction, possibly caused by the presence of these large Fe aggregates.

This chapter is based on:

Solsona, M.*, Nieuwelink, A-E.*, Meirer, F., Abelmann, L., Odijk, M., Olthuis, W., Weckhuysen, B.M., van den Berg, A., Magnetophoretic Sorting of Single Catalyst Particles. Angewandte ChemieInternational Edition), 57, 10589-10594, (2018).

* Both authors contributed equally to this work 


\subsection{Introduction}

Recent advances in microfluidics open new ways to work with microsized structures, such as individual cells and particles. ${ }^{1-4}$ The small volumes and high-speed regimes where microfluidics work, allow experiments at the single particle level and in a high-throughput fashion, as well as better control on the parameters affecting the system. ${ }^{5-10}$ Different fields have benefitted from microfluidics, however, for catalysis it is far from its full potential. For example, microfluidics has been used for the monitoring of chemical reactions ${ }^{11,12}$ and the synthesis of catalysts, offering a better control on the temperature and mixing of reagents, resulting in more homogeneously shaped catalysts. ${ }^{13,14}$ However, a great potential of microfluidics still lies in the separation of single catalyst particles. Microfluidics offers various techniques to separate particles based on their physical properties. For example, passive methods like Pinched Flow Fractionation and Dean Flow Fractionation make use of the inertial properties of particles and fluids in microchannels to sort them by size or shape. On the other hand, active sorting methods make use of external electric, magnetic, optical or acoustic fields to continuously separate particles or cells.

A catalytic system that is very suitable as a showcase for single particle sorting using microfluidics, is the Fluid Catalytic Cracking (FCC) catalyst. ${ }^{15-17}$ FCC particles are 50-150 micrometre sized, multi-component porous particles and responsible for cracking long-chain hydrocarbons into gasoline and base chemicals, such as propylene. ${ }^{18,19}$ During the FCC process, the catalyst deactivates irreversibly due to dealumination and the accumulation of metals, mainly iron (Fe), nickel (Ni) and vanadium (V). While Ni and V decrease the yield by promoting other reactions like coke formation, Fe is supposed to deactivate the particles by creating a $\sim 1 \mu \mathrm{m}$ shell of reduced porosity on the particles' outer surface, either via vitrification of the particle matrix in the presence of Fe and/or co-deposition of silica. ${ }^{20-29}$ Therefore, during operation, to create a stable process efficiency, a small fraction of catalyst is constantly being replaced, resulting in a mixture called Equilibrium catalyst (ECAT). To investigate their deactivation, FCC ECAT particles are often sorted based on their skeletal density, which is associated with metal loading and age. ${ }^{30}$ Although this technique gives a clear negative correlation between skeletal density and catalytic activity, it is a batch process that does not give insight in the individual role of the different metals that deactivate FCC particles. Therefore, there is a need for a new kind of sorting method, that is, a tool able to sort FCC ECAT particles by just one of the metals that deactivate them.

In this chapter, we present for the first time a 3-D printed magnetophoretic chip, ${ }^{31}$ as outlined in Figure 3.1a, which is able to sort individual catalyst particles by their magnetic moment. The sorted FCC particles were subsequently analysed by X-ray fluorescence microscopy (Figure 3.1c), fluorescence micro-spectroscopy (Figure 3.1d), magnetic hysteresis and 
field cooled magnetic measurements (Figure 3.1b) to explain the origin of the sorting principle. Magnetophoresis can be used to sort magnetic and nonmagnetic particles and fluids (ferrofluids) ${ }^{32}$, and has been previously used to sort cells and magnetic particles. ${ }^{33-38}$ This very reproducible approach could also be used in other magnetic catalysts such as $\mathrm{Ni}$ or Co.

Previous work has demonstrated (at least) two types of Fe distributed in FCC particles: Fe inside the clay components of the catalyst and Fe deposited during the FCC process, which is mainly accumulated in the first two micrometres of the outer surface of the particle. ${ }^{18}$ This deposited Fe, which originates from the feedstock or from reactor debris, is mainly held responsible for the deactivation of these catalysts due to pore blocking. ${ }^{20,28,29,41}$ Furthermore, it was shown that these two types of Fe distributions have different oxidation states. Inside the clay, $\mathrm{Fe}$ is found as $\mathrm{Fe}^{0}$ and as $\mathrm{Fe}^{\mathrm{II}}$ in the form of $\mathrm{FeO}$ and $\mathrm{SiFeO}_{3}{ }^{28}$ On the other hand, $\mathrm{Fe}$ III is found on/in the outer FCC particle layer.

FCC catalyst particles were previously sorted by magnetic fields in order to decrease the disposal of spent catalyst in FCC reactors. This industrial approach consisted of a permanent rolling magnet cylinder under a belt where particles with different sizes were fed on, to sort them in just two fractions. ${ }^{42}$ In this work, however, the particles have been pre-sorted by size and we focus on the relation of Fe content, magnetic properties and the availability of acid sites ${ }^{43-}$ 46 in a wide range of Fe content and on real, industrially processed FCC ECAT particles, providing a unique catalyst diagnostics platform for single particle analysis.

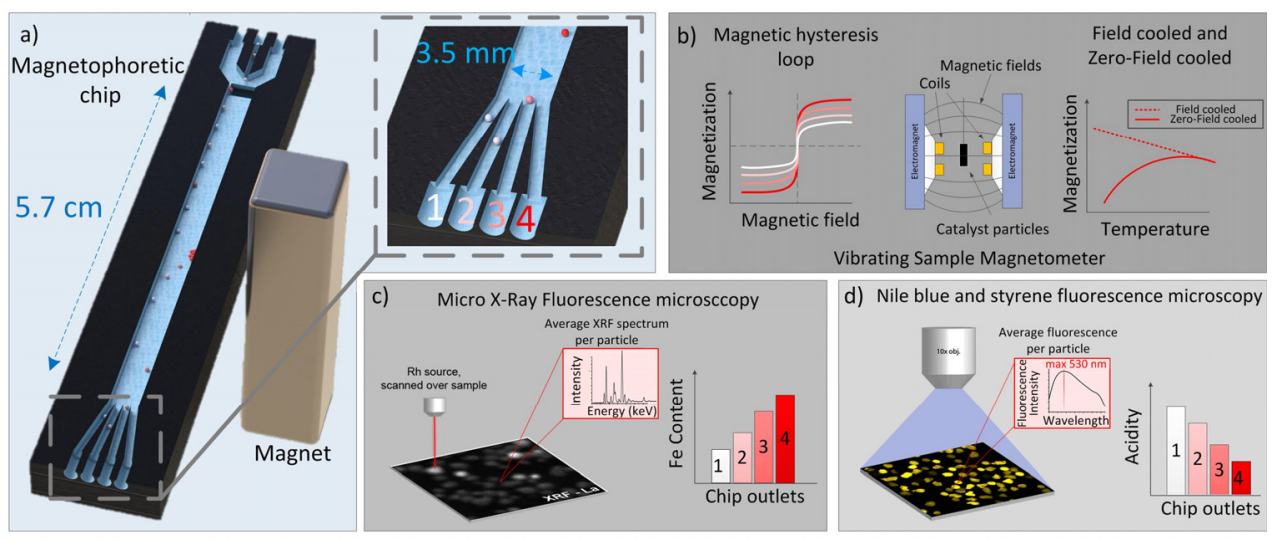

Figure 3.1. (a) Drawing of the inside channel of the 3-D printed microfluidic chip for sorting catalyst particles. (b) Sketch of the vibrating sample magnetometer, invented hysteresis loops of the different outlets and zero-field cooled and field cooled measurements. (c) micro X-ray fluorescence and (d) fluorescence micro-spectroscopy to analyse the sorted FCC catalyst particles. 


\subsection{Materials and methods}

A 3-D printed chip was designed in SolidWorks and printed with a Formlabs Form 2 printer. A chip holder was fabricated with black delrin (polyacetal) and brass; and it consisted of a fixed and mobile part where the microfluidic chip and the magnet were fixated, respectively.

The FCC ECAT particles were sorted with a filter $(70-90 \mu \mathrm{m})$ and calcined at $600{ }^{\circ} \mathrm{C}\left(1{ }^{\circ} \mathrm{C} / \mathrm{min}\right.$ to $120{ }^{\circ} \mathrm{C}$ followed by $\left.5{ }^{\circ} \mathrm{C} / \mathrm{min}\right)$ to remove all remaining coke that could be present on the catalyst particles. In order to adapt the viscosity and thereby the residence time of the particles in the magnetic field, calcined FCC particles were introduced in a mixture of glycerol and water 1:3 vol., in an open vertical syringe and stirred to avoid excessive sedimentation. An $1 \times 1 \times 4 \mathrm{~cm}$ Neodymium magnet with a remanence of $1.23 \mathrm{~T}$ purchased from Supermagnete was fixed in the chip holder mobile part and approached to the chip at a known distance. Four $50 \mathrm{ml}$ syringes were used with three Nemesys BASE 120 and one Harvard apparatus PHD 2000 programmable syringe pumps with flows of 120, 120, 30 and $800 \mu 1(\mathrm{~min})^{-1}$. A Grasshopper3 GS3-U3-23S6M high-speed camera was used to record the trajectories of the FCC ECAT particles inside the chip.

A small portion of the sorted FCC ECAT particles was placed between two $5 \times 5 \mathrm{~mm}$ tape layers and introduced in a Quantum Design ${ }^{\circledR}$ PPMS-VSM (Physical property measurement system vibrating sample magnetometer) to measure their magnetic moment. The scan rate was $0.02 \mathrm{~T}$ per second from $9 \mathrm{~T}$ to $-9 \mathrm{~T}$ and up to $9 \mathrm{~T}$ again at room temperature. The small step at $5 \mathrm{~T}$, visible in all loops, is attributed to the PPMS and not to the sample. This was demonstrated by recognizing the same step in a measurement with no sample, illustrated in Figure A.3 in Appendix. The diamagnetic contribution was extracted from the samples until a plateau at high magnetic fields was observed.

For the EPR measurements, dried and calcined particles of fraction F5 were measured with a Bruker EMX Plus 6000 Gauss spectrometer operating with an ER 041 XG X-band microwave frequency of $9.5 \mathrm{GHz}$. For the UV-vis microscopy spectra, particles from fraction F5 with visible clusters were selected. With a CRAIC 20/30 PVTM micro-spectrophotometer in reflectance mode, equipped with a $74 \times 0.65 \mathrm{NA}$ reflective lens and $30 \mathrm{~W}$ halogen lamp, spectra were collected from a spot indicated with a black square in the images in Figure A.6b in Appendix.

Sorted FCC ECAT particles were introduced in Ge Varnish stuck on a $5 \mathrm{x}$ $5 \mathrm{~mm}$ silicon holder. Afterwards, the sample was introduced in the Quantum Design ${ }^{\circledR}$ PPMS-VSM. ZFC: The sample was cooled down until $10 \mathrm{~K}$ at 0 Oe and heated up until $305 \mathrm{~K}$ at $500 \mathrm{Oe}$. FC: a magnetic field of 500 Oe was applied and the sample was cooled down until $10 \mathrm{~K}$. Then, the magnetic field was set to 0 Oe and the samples were heated up until $305 \mathrm{~K}$. 
For the acidity and metal loading characterization of the catalyst particles, the magnetically sorted FCC ECAT fractions were carefully dried and calcined in air by placing them in a ceramic crucible. The samples were heated to $120{ }^{\circ} \mathrm{C}$ (ramp $1{ }^{\circ} \mathrm{C} / \mathrm{min}$ ) for $1 \mathrm{~h}$ to remove water and then heated to $600{ }^{\circ} \mathrm{C}$ (ramp 5 ${ }^{\circ} \mathrm{C} / \mathrm{min}$ ) for $5 \mathrm{~h}$. For the Brønsted acidity measurements, the separated fractions of catalyst particles were placed on sticky tape. A droplet of 4-methoxystyrene was placed next to the tape and the sample was heated to $100{ }^{\circ} \mathrm{C}$ for $15 \mathrm{~min}$. The sample was covered and via diffusion, the 4-methoxystyrene (Sigma Aldrich, $97 \%$ ) could enter the pores of the FCC particles. With a Nikon A1 confocal fluorescence microscope (10x objective) equipped with a $488 \mathrm{~nm}$ laser, the fluorescence intensity of the FCC ECAT particles was measured. Details of these staining experiments can be found in literature.39,40 For pore accessibility measurements, the separated FCC ECAT fractions were added to a solution of $0.1 \mathrm{wt} \%$ Nile blue A (Acros Organics, pure) in ethanol (Sigma Aldrich, 100\%). After equilibrating for $1 \mathrm{~h}$, the catalyst particles were placed on sticky tape and analysed with the same confocal fluorescence microscope using a $561 \mathrm{~nm}$ laser.

The different fractions of sorted FCC ECAT particles where also characterized with micro-X-ray fluorescence (XRF) to determine the presence of metals in the catalyst particles. For the micro-XRF measurements, the samples that were used for the 4-methoxystyrene staining were dried carefully and mounted in an Orbis PC SDD with a Rh-tube as X-ray source (30 kV and 200 $\mathrm{nA}$ ). With a spot size of $30 \mu \mathrm{m}$ and a step size of $15 \mu \mathrm{m}$ the surface was scanned (300 ms integration time) to obtain an XRF map with the highest intensity per pixel for every element.

The developed 3-D printed microfluidic chip consists of 5 inlets (4 inlets to focus the particles inside the chip and 1 inlet to introduce the particles), a straight channel of $5.7 \mathrm{~cm}$ long, $3.5 \mathrm{~mm}$ wide and $1 \mathrm{~mm}$ deep where the particles get attracted to the magnet, and 4 outlets (Figure 3.1a, an image of the microfluidic chip can be found in Figure A.1 in Appendix). Considering the purpose of the device to sort by Fe content and knowing that the magnetic force is related to Fe volume (i.e. total mass of Fe in the particle), FCC ECAT particles were size pre-sorted with two sieves in a range or 70-90 $\mu \mathrm{m}$, to have a similar Fe concentration per particle in each fraction. Based on the large differences between magnetic moments found for particles of this FCC ECAT, they were sorted into 5 fractions; four microfluidic outlets and a fifth fraction formed by the particles that adhered to the wall of the channel at the position of the magnet. 


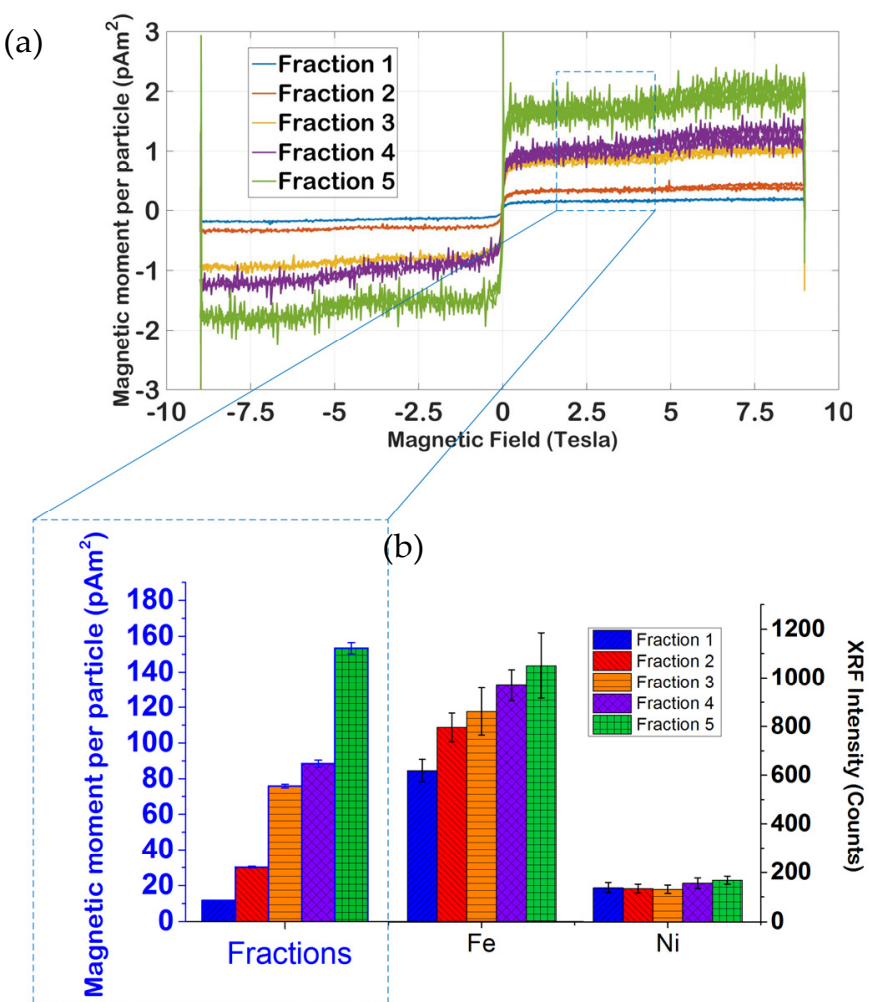

Figure 3.2. (a) Hysteresis loops of the sorted FCC ECAT particles per outlet and per particle. Number of particles per outlet (Outlet 1: 466, Outlet 2: 255, Outlet 3: 120, Outlet 4: 80 and Outlet 5: 44 particles), (b) (left axis) relative XRF Fe and Ni intensities and (right axis) magnetic moment per fraction of the sorted single FCC ECAT particle.

\subsection{Results and discussion}

\subsubsection{Magnetic properties and composition of sorted FCC particles}

To know the magnetic properties of the sorted FCC particles, their magnetic moment was measured and the results are shown in Figure 3.2a. For further information on the theory on superparamagnetism used in this study we refer to Section A.1 in Appendix. Hysteresis loops per fraction 1 to 5 and averaged per single FCC ECAT particle show a superparamagnetic behaviour, no significant coercivity and an increasing moment per particle with increasing fraction number. This is an indication for either an increase in the amount of magnetic material inside the catalyst particles and/or a change in form or oxidation state of certain magnetic materials. Consequently, micro-XRF measurements were performed by qualitatively mapping the elemental composition of the particles in all sorted fractions, see Figure A.4 in Appendix. Figure $3.2 \mathrm{~b}$ shows an increased average Fe content in the FCC ECAT particles from fraction 1 to 5 , confirming that $\mathrm{Fe}$ is the main actor on the magnetic attraction to the magnet. Also, when Fe increases by a factor of 2 the magnetic 
moment increases by a factor of 13 . This seems to point to a change in form and/or oxidation state. Importantly, Figure A.4 in Appendix shows that all particles have a similar La content ${ }^{47}$ (a marker for zeolite US-Y) and thus a similar amount of zeolite per fraction, the active site in FCC. In FCC deactivation research, Ni can be used as a measure for the age of an FCC particle, since its concentration increases homogeneously with deactivation of the FCC particles and, in contrast to Fe, is not present in fresh FCC particles. ${ }^{15,48,49}$ However, this FCC ECAT in general has a low Ni content, therefore there are no clear trends observed that link our magnetophoretic sorting to particle age.

When taking a closer look at the FCC particles in the five fractions see Figure 3.3a, aggregates were observed on the surface of the particles of fractions 2 to 5. SEM-EDX measurements (Figures A.5 in Appendix) revealed that both black and orange aggregates consist of iron oxide. Bulk EPR and UV-vis microscopy results on the aggregates (Figure A.6 in Appendix), indicate the presence of different forms of $\mathrm{Fe}_{2} \mathrm{O}_{3}$ or $\mathrm{FeO}(\mathrm{OH})$. In Figure 3.3b, the percentage of the particles' visible surface (projected to 2- D) that is covered by these aggregates is listed per fraction. To do this, the images were binarized with a manually set threshold to count the area of the aggregates and particles per 2-D image. The percentage of the 2-D visible particle surface that was found covered by black or red aggregates was then calculated showing an increasing covered area from fractions 2 to 5 .

(a)

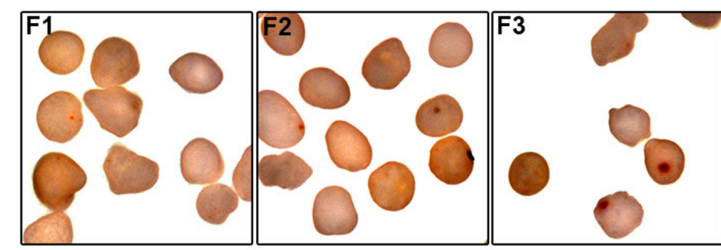

(b)

\begin{tabular}{|r|c|c|}
\hline & $\begin{array}{r}\text { area \% with } \\
\text { aggregates }\end{array}$ & \# particles \\
\hline F1 & 0.029 & 200 \\
\hline F2 & 0.030 & 193 \\
\hline F3 & 0.14 & 94 \\
\hline F4 & 0.37 & 149 \\
\hline F5 & 1.4 & 73 \\
\hline
\end{tabular}

(c)

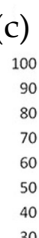

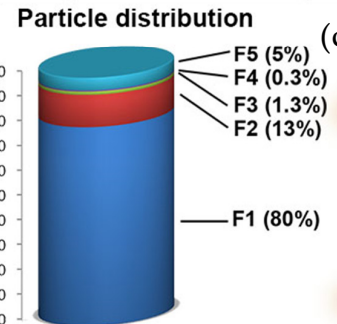

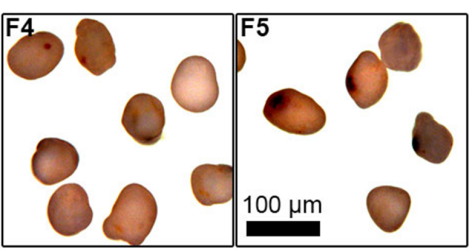

(d)
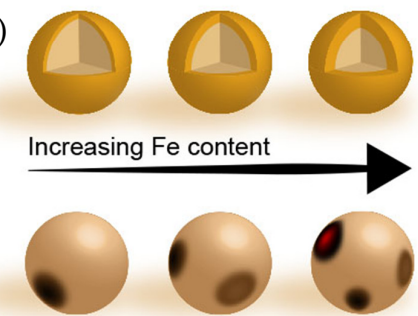

Figure 3.3. Optical images (a) of the five magnetically sorted FCC ECAT fractions show an increasing number of dark spots on their surface with increasing magnetic moment. The table (b) shows the ratio of aggregate area to particle area for each fraction. In (c) the particle distribution is shown. In (d) a schematic representation is given of the formation of a Fe shell or Fe clusters on the surface of FCC ECAT particles.

As previously mentioned, it is generally accepted that Fe coming from the crude oil feedstock is deposited mainly in/on the outer rim of the FCC particle, as schematically illustrated in Figure 3.3 d. $^{20,24}$ The FCC catalyst particle 
distribution over the five fractions in Figure 3.3c indeed shows that $80 \%$ of the FCC ECAT particles, which are those in fraction 1, follow this pathway for the deposition of Fe. However, the catalyst particles in fractions 2 to 5, which add up to $20 \%$ of the whole FCC ECAT sample, contain an increasing amount of Fe aggregates on their surface, as illustrated in Figure 3.3d. When magnetic material is present in bigger Fe oxide clusters, the magnetic moment at lower fields is higher. This effect could cause a larger deviation in the Fe content per fraction. For that, a further study on previously shown hysteresis loops by performing a Langevin fit was performed (see Equation 1 in Section A.1 and Sections A.8 and A.9 in Appendix). For further information on the theory on superparamagnetism used in this study we refer to Section A.1 in Appendix. It was found that there are no big differences between the average cluster sizes of each fraction.

To calculate the range of average cluster sizes inside the FCC ECAT particles and their anisotropy constant, the zero-field cooled (ZFC) and fieldcooled (FC) technique were used. With this technique the magnetic moment of a sample is measured when heating with (FC) and without (ZFC) previously applied magnetic field. From the temperature at which the ZFC moment coincides with the FC, which is the blocking temperature $\mathrm{T}_{\mathrm{B}}$, and taking $\mu\left(K_{B} T\right)^{-1}$ from the Langevin fitting, the average cluster size and constant anisotropy can be extracted (see, Equations 5 and 6 in Section A.1 in Appendix).
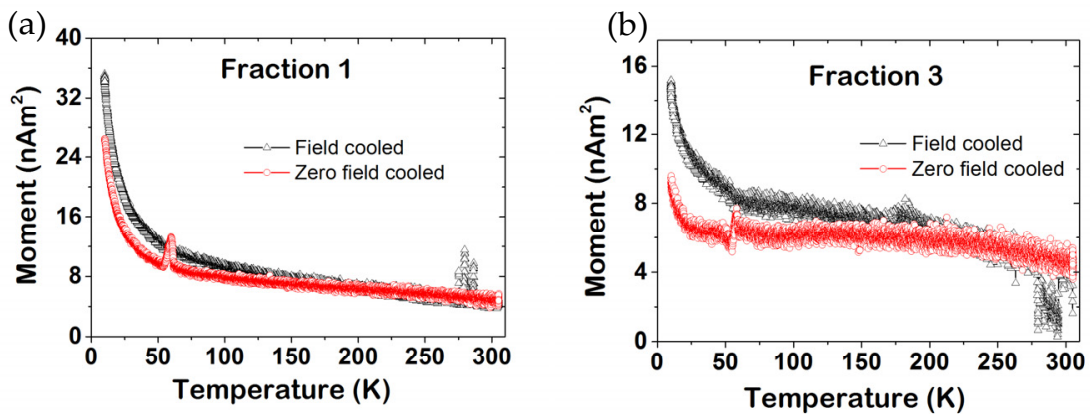

(c)

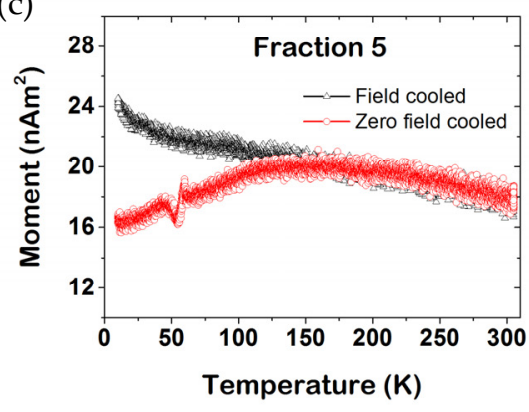

Figure 3.4. ZFC and FC experimental result of sorted FCC ECAT $(a) \approx 150$ particles from fraction $5,(b) \approx 100$ particles from fraction 3 and $(c) \approx 500$ particles from fraction 1 . 
Figure 3.4 presents the ZFC and FC experimental results of fractions 1, 3 and 5, showing very different behaviours at temperatures below $50 \mathrm{~K}$. However, when comparing $\mathrm{T}_{\mathrm{B}}$, no significant differences between the fractions are observed. Taking the $\mathrm{T}_{в}$ of fraction 5 as $150 \mathrm{~K}$, we find the ratio of $\mathrm{K}(\mathrm{Ms})^{-1}$ to be 0.23 . This value is 10 times higher than of typical magnetic iron oxides of the form of magnetite and maghemite, 0.04 and 0.02, respectively. Assuming that in fraction 5 the Fe content is $3 \mathrm{wt} \%$, similar to the HML FCC particles from Meirer et $a^{23}$, the anisotropy constant and the saturation magnetization can be calculated (see Section A.1) resulting in $17 \mathrm{~kJ} / \mathrm{m}^{3}$ and $73 \mathrm{kA}(\mathrm{m})^{-1}$; much lower than that of maghemite and magnetite. We can also calculate the average radius of the clusters to be $9 \mathrm{~nm}$. This confirms that during the FCC process most of the Fe is deposited as clusters with sizes of a few $\mathrm{nm}$, big orange and black Fe aggregates included. Considering that, in fractions 1 and 5, when Fe content increases with a factor of 2 the magnetic moment increases with a factor of 13 and that FC measurements show very different behaviours at low temperature, it is suggested that Fe deposited during the FCC process is in another ${ }^{20,28}$ and more magnetic form. The complexity and number of Fe and $\mathrm{Fe}_{x} \mathrm{O}_{\mathrm{y}}$ types and knowing that the magnetic moment of Fractions 2-5 could be caused by a combination of these different forms only allows for speculative explanation about its final form.

\subsubsection{Acidity and accessibility of sorted FCC particles}

To verify whether these clusters are related to a decrease in the amount of available acid sites (after this referred to as acidity) due to pore blocking, staining experiments were performed using 4-methoxystyrene and Nile blue A. The oligomerization of 4-methoxystyrene is catalysed by the Brønsted acid sites in zeolite domains and induces fluorescence. ${ }^{40,45,46}$ This fluorescence was used as a measure for acidity by averaging the fluorescence intensity per particle over \pm 100 catalyst particles for all five fractions. These average intensities were compared with Nile blue A staining results. In contrast to 4-methoxystyrene which reacts within the zeolite, Nile blue $\mathrm{A}$ is a conjugated molecule that can only enter the meso- and macroporous structure of FCC catalyst particles, see Figure 3.5a. ${ }^{45}$ Therefore, it can be used as an indication for the degree of pore accessibility. It is expected that with increasing metal accumulation, both the acidity and the pore accessibility drop. ${ }^{23}$ However, when we look at the accessibility and acidity of the magnetically sorted fractions we see a different behaviour. In order to assess this in more detail we performed staining experiments with 4-methoxystyrene and Nile Blue A in combination with confocal fluorescence microscopy and details of these experiments can be found in Figure A.9 in Appendix A. 
Figure $3.5 \mathrm{~b}$ shows the distribution of fluorescence intensities of 4methoxystyrene and Nile Blue A per fraction. A single particle analysis was performed by placing multiple particles in the field of view of the fluorescence microscope, see Figure 3.5). From each particle, an average fluorescence spectrum was measured and the emission maximum was used to create intensity histograms for both staining agents per fraction. In general, the spread within all the fractions is large, meaning that every fraction still contains very active and deactivated particles. In Figure 3.5c and $5 d$, it can be seen that in fraction 1 , containing $80 \%$ of all FCC ECAT particles, most particles show a high fluorescence signal, which can be related to a high acidity. Although a very clear decrease in acidity is detected, the particles in the fractions with higher Fe content are not all deactivated, see Figure A.9 in Appendix A10. Furthermore, there is no significant change in the accessibility histograms. For example, in fraction 4, there are still ECAT particles with high acidity and accessibility; in fact, the particles with Fe aggregates on the surface show very high fluorescence intensity. This means that when an aggregate of Fe is present, the rest of the particle can still be accessible for catalysis. This indicates that the presence of Fe aggregates does not automatically imply the presence of Fe deposited as a shell. The decrease in acidity can be caused by other deactivating processes like, for example, dealumination. ${ }^{47}$

\subsection{Conclusions}

In conclusion, a newly developed 3-D printed microfluidic chip has been used to sort a set of FCC ECAT particles magnetophoretically into 5 fractions, based on their magnetic moment. We believe that this technology could also be used to sort other magnetic catalysts for analysis purposes, for example FischerTropsch catalysts. It can serve as a tool to decrease the heterogeneity of samples. The trends in magnetic moment of the sorted FCC particles correlate with Fe content; when Fe content increases by a factor of 2 the magnetic moment increases by a factor of 13. The magnetic properties of the FCC particles, extracted by a Langevin fitting show that their magnetic susceptibility doesn't vary between the fractions. Also, low temperature magnetization measurements show different behaviours at low temperatures but similar blocking temperature between the fractions. These magnetic observations strongly point into the direction that variation in magnetic moment is caused by an increased number, different form but similar size of iron-containing nanoclusters. In addition, on average, when the Fe content increases, catalyst particle acidity generally decreases. However, the spread within acidity of each fraction is still large: not all highly magnetic particles show a high degree of deactivation. Many of the highly magnetic particles contain large Fe oxide aggregates that leave the rest of the particle accessible for reaction. 
(a)

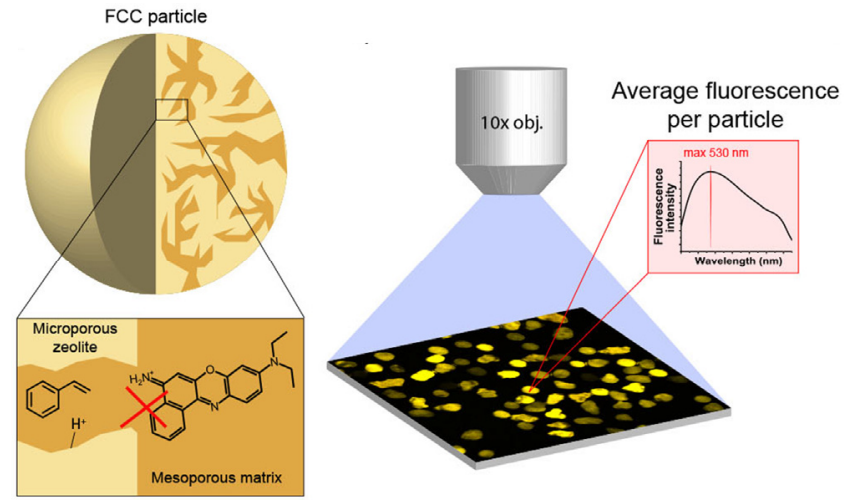

(b)

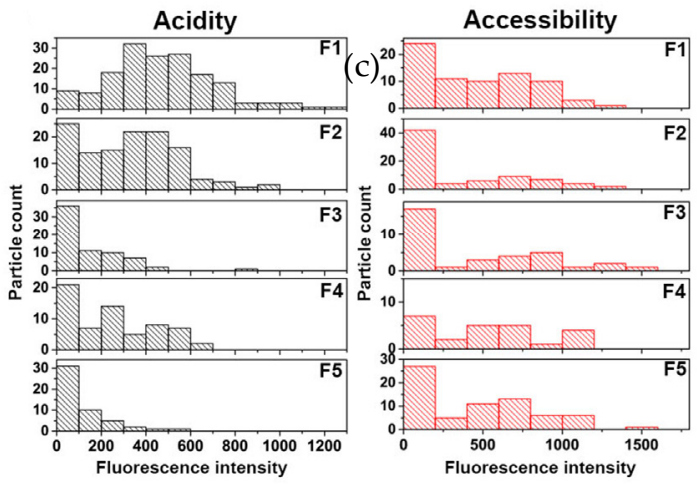

Figure 3.5. Schematic representation (a) of Nile blue and styrene staining, indicating the zeolite shape selectivity that prevents the large Nile blue A from entering the zeolites' microporous structure. Fluorescence micro-spectroscopy images $(b)$ were collected and analysed per particle. Details can be found in Figure A.9 in Appendix A. Histograms of the average fluorescence intensity per FCC ECAT particle of (c) 4-methoxystyrene oligomers as a measure for acidity and (d) Nile blue as a measure for pore accessibility.

\subsection{References}

1. Bhagat, A. A. S. et al. Microfluidics for cell separation. Med. Biol. Eng. Comput. 48, 999-1014 (2010).

2. Wyatt Shields IV, C., Reyes, C. D. \& López, G. P. Microfluidic cell sorting: a review of the advances in the separation of cells from debulking to rare cell isolation. Lab Chip 15, 1230-1249 (2015).

3. Gossett, D. R. et al. Label-free cell separation and sorting in microfluidic systems. Anal. Bioanal. Chem. 397, 3249-3267 (2010).

4. Valero, A. et al. Miniaturisation for chemistry , biology \& bioengineering Gene transfer and protein dynamics in stem cells using single cell electroporation in a microfluidic device $\{.8$, (2008).

5. Tiggelaar, R. M. et al. Fabrication, mechanical testing and application of high-pressure glass microreactor chips. Chem. Eng. J. 131, 163-170 (2007). 
6. Male, P. Van, Croon, M. H. J. M. De, Tiggelaar, R. M., Berg, A. Van Den $\&$ Schouten, J. C. Heat and mass transfer in a square microchannel with asymmetric heating. 47, 87-99 (2004).

7. Doku, G. N., Verboom, W., Reinhoudt, N. \& Berg, A. Van Den. Onmicrochip multiphase chemistry - a review of microreactor design principles and reagent contacting modes. Tetrahedron 61, 2733-2742 (2005).

8. Wensink, H. et al. Measuring reaction kinetics in a lab-on-a-chip by microcoil NMR. 280-284 (2005). doi:10.1039/b414832k

9. Marre, S. \& Jensen, K. F. Synthesis of micro and nanostructures in microfluidic systems. Chem. Soc. Rev. 39, 1183 (2010).

10. Tokeshi, M. et al. Continuous-flow chemical processing on a microchip by combining microunit operations and a multiphase flow network. Anal. Chem. 74, 1565-1571 (2002).

11. Meier, T.-A. et al. On-chip monitoring of chemical syntheses in microdroplets via surface-enhanced Raman spectroscopy. Chem. Commun. 51, 8588-8591 (2015).

12. Chan, K. L. A., Gulati, S., Edel, J. B., de Mello, A. J. \& Kazarian, S. G. Chemical imaging of microfluidic flows using ATR-FTIR spectroscopy. Lab Chip 9, 2909 (2009).

13. Kim, Y. H. et al. Droplet-based microreactors for continuous production of palladium nanocrystals with controlled sizes and shapes. Small 9, 3462-3467 (2013).

14. Yao, X., Zhang, Y., Du, L., Liu, J. \& Yao, J. Review of the applications of microreactors. Renew. Sustain. Energy Rev. 47, 519-539 (2015).

15. Vogt, E. T. C. \& Weckhuysen, B. M. Fluid catalytic cracking : recent developments on the grand old lady of zeolite catalysis. Chem. Soc. Rev. 44, 7342-7370 (2015).

16. Cerqueira, H. S., Caeiro, G., Costa, L. \& Ribeiro, F. R. Deactivation of FCC catalysts. J. Mol. Catal. A Chem. 292, 1-13 (2008).

17. Ertl, G., Knözinger, H., Schöth, F. \& Weitkamp, J. Handbook of Heterogeneous Catalysis, 8 Volumes, 2nd Edition. (2008).

18. Komvokis, V., Tan, L. X. L., Clough, M., Pan, S. S. \& Yilmaz, B. Zeolites in Fluid Catalytic Cracking (FCC). in Zeolites in Sustainable Chemistry 271297 (Springer, 2016).

19. Sadeghbeigi, R. Fluid Catalytic Cracking Handbook. An expert guide to the practical operation, design and optimization of FCC units. (Elsevier).

20. Wise, A. M. et al. Nanoscale Chemical Imaging of an Individual Catalyst Particle with Soft X-ray Ptychography. ACS Catal. 6, 2178-2181 (2016).

21. De Winter, D. A. M., Meirer, F. \& Weckhuysen, B. M. FIB-SEM Tomography Probes the Mesoscale Pore Space of an Individual Catalytic Cracking Particle. ACS Catal. 6, 3158-3167 (2016).

22. Mitchell, B. I. R. Metal Contamination of Cracking Catalysts. Ind. Eng. 
Chem. Prod. Res. Dev. 19, 209-213 (1980).

23. Meirer, F. et al. Life and death of a single catalytic cracking particle. Sci. Adv. 1, e1400199 (2015).

24. Meirer, F. et al. Mapping metals incorporation of a whole single catalyst particle using element specific X-ray nanotomography. J. Am. Chem. Soc. 137, 102-105 (2015).

25. Meirer, F. et al. Agglutination of single catalyst particles during fluid catalytic cracking as observed by X-ray nanotomography. Chem. Commun. 51, 8097-8100 (2015).

26. Kalirai, S., Boesenberg, U., Falkenberg, G., Meirer, F. \& Weckhuysen, B. M. X-ray Fluorescence Tomography of Aged Fluid-Catalytic-Cracking Catalyst Particles Reveals Insight into Metal Deposition Processes. ChemCatChem 7, 3674-3682 (2015).

27. Liu, Y., Meirer, F., Krest, C. M., Webb, S. \& Weckhuysen, B. M. Relating structure and composition with accessibility of a single catalyst particle using correlative 3-dimensional micro-spectroscopy. Nat. Commun. 7:12634, (2016).

28. Ihli, J. et al. Localization and Speciation of Iron Impurities within a Deactivated Fluid Catalytic Cracking Catalyst. Angew. Chem. Int. Ed. 56, 1-6 (2017).

29. Ihli, J. et al. A three-dimensional view of structural changes caused by deactivation of fluid catalytic cracking catalysts. Nat. Commun. 8, (2017).

30. Dyrkacz, G. R., Ruscic, L., Marshall, C. L. \& Reagan, W. Separation and Characterization of FCC Catalysts Using Density Gradient Separation. Energy \& Fuels 71, 849-854 (2000).

31. Au, A. K., Huynh, W., Horowitz, L. F. \& Folch, A. 3D-Printed Microfluidics. Angew. Chem. Int. Ed. 55, 3862-3881 (2016).

32. Gao, Y., Jian, Y. C., Zhang, L. F. \& Huang, J. P. Magnetophoresis of nonmagnetic particles in ferrofluids. J. Phys. Chem. C 111, 10785-10791 (2007).

33. Pamme, N. Magnetism and microfluidics. Lab Chip 6, 24-38 (2006).

34. Pamme, N. \& Manz, A. On-chip free-flow magnetophoresis: Continuous flow separation of magnetic particles and agglomerates. Anal. Chem. 76, 7250-7256 (2004).

35. Martin A. M. Gijs, F. L. and U. L. Microfluidic Applications of Magnetic Particles for Biological Analysis and Catalysis. Chem. Rev. 110, 1518-1563 (2010).

36. Pamme, N. \& Wilhelm, C. Continuous sorting of magnetic cells via onchip free-flow magnetophoresis. Lab Chip 6, 974-980 (2006).

37. Nezihi Murat Karabacak, Philipp S Spuhler, Fabio Fachin, Eugene J Lim, Vincent Pai, Emre Ozkumur, Joseph M Martel, Nikola Kojic, Kyle Smith, Pin-i Chen, Jennifer Yang, Henry Hwang, Bailey Morgan, Julie Trautwein, Thomas A Barber, Shannon L Stott, Shyamala, D. A. H. \& M. 
T. Microfluidic, marker-free isolation of circulating tumor cells from blood samples. Nat. Protoc. 9, 694-710 (2014).

38. Furlani, E. P. Analysis of particle transport in a magnetophoretic microsystem. J. Appl. Phys. 99, 1-35 (2006).

39. Kerssens, M. M. M. et al. Photo-spectroscopy of mixtures of catalyst particles reveals their age and type. Faraday Discuss. 0, 1-11 (2016).

40. Buurmans, I. L. C. et al. Staining of fluid-catalytic-cracking catalysts: Localising Brønsted acidity within a single catalyst particle. Chem. - A Eur. J. 18, 1094-1101 (2012).

41. Mance, D. et al. A DNP-supported solid-state NMR study of carbon species in fluid catalytic cracking catalysts. Chem. Commun. 53, 3933-3936 (2017).

42. Goolsby, T. L. \& Moore, H. F. Development of FCC Catalyst Magnetic Separation. Sep. Sci. Technol. 32, 655-668 (1997).

43. Aramburo, L. R. et al. Styrene oligomerization as a molecular probe reaction for Brønsted acidity at the nanoscale. Phys. Chem. Chem. Phys. 14, 6967-6973 (2012).

44. Ristanovic, Z. et al. High-Resolution Single-Molecule Fluorescence Imaging of Zeolite Aggregates within Real-Life Fluid Catalytic Cracking Particles. Angew. Chem. Int. Ed. 54, 1836-1840 (2015).

45. Buurmans, I. L. C. et al. Catalytic activity in individual cracking catalyst particles imaged throughout different life stages by selective staining. Nat. Chem. 3, 862-867 (2011).

46. Kerssens, M. M., Wilbers, A., Kramer, J., Peinder, P. De \& Mesu, G. Photo-Spectroscopy of Mixtures of Catalyst Particles Reveals their Age and Type. Faraday Discuss. 188, 69-79 (2016).

47. Kalirai, S., Paalanen, P. P., Wang, J., Meirer, F. \& Weckhuysen, B. M. Visualizing Dealumination of a Single Zeolite Domain in a Real-Life Catalytic Cracking Particle. Angew. Chem. Int. Ed. 55, 11134-11138 (2016).

48. Kugler, E. L. \& Leta, D. P. Nickel and Vanadium on Equilibrium Cracking Catalysts Secondary Ion Mass Spectrometry by Imaging. J. Catal. 395, 387-395 (1988).

49. Escobar, A. S., Pereira, M. M., Pimenta, R. D. M., Lau, L. Y. \& Cerqueira, H. S. Interaction between $\mathrm{Ni}$ and $\mathrm{V}$ with USHY and rare earth HY zeolite during hydrothermal deactivation. Appl. Catal. A Gen. 286, 196-201 (2005). 


\section{Chapter

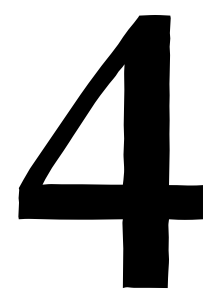

\section{First Steps Towards a Particle Sorting System using the Magnus Force}

Tn this chapter, we present the follow up towards a magnetophoretic activity sorter of single catalyst particles. Fluid catalytic cracking (FCC) particles deactivate amongst other processes, due to Fe deposition. In the previous chapter, we showed that some of the sorted particles with high Fe content nevertheless had high activity. This is supposedly caused by the agglomeration of Fe leaving the rest of the catalyst still active. Therefore, new ways to sort particles by their Fe distribution are needed in order to develop an activity sorter. In this work, we propose to use a rotating magnetic field to spin FCC particles with inhomogeneous magnetic material. By spinning the particles, a force will deflect them by the Magnus effect. Here, we show the feasibility of using the Magnus force to deflect particles with a diameter of $\approx 90 \mu \mathrm{m}$. 


\subsection{Introduction}

As stated in the previous chapter, Fluid Catalytic Cracking (FCC) particles are porous materials responsible for cracking the long chain hydrocarbons in the crude oil industry. ${ }^{1}$ However, these catalysts deactivate due to, amongst other processes, Fe coming from the feedstock. ${ }^{2-4}$ Chapter 3 presented a single catalytic particle magnetophoretic sorter that was used to sort FCC particles by their Fe content. ${ }^{5}$ Activity tests of sorted FCC particles showed that not all the particles with a large concentration of Fe have a lower activity. First assumptions pointed to the direction that the inhomogeneous Fe distribution within those particle lets some parts of the catalyst remain active which can disturb the relation between the activity and the Fe content of the FCC particles after the magnetophoretic sorting. Therefore, new methods to sort FCC particles by their Fe distribution are necessary to convert the magnetophoretic microfluidic chip into a direct activity sorter.

When a spherical microparticle has a magnetic material within it, a torque can be applied with a magnetic field across the particle. Therefore, if the magnetic field rotates the particles will spin and try to follow it. However, this is not the case for particles with homogeneous or isotropically shaped magnetic materials because the energy needed to rotate them is higher than the energy needed for the dipole to align with the new direction of the rotating magnetic field. ${ }^{6}$ Therefore, if a rotating magnetic field is applied to the magnetophoretically sorted particles, just those with inhomogeneous Fe distribution or anisotropic shape will rotate with the magnetic field.

During the last couple of decades microfluidic technologies have been extensively used to sort cells and microparticles based on, amongst others, their size, density, shape or dielectric properties. ${ }^{7-13}$ Many studies have focused on the forces acting on the cells or microparticles, such as: gravity, wall-induced, rotational, Saffman or shear-induced, or drag force. ${ }^{12,14}$ The combination of these forces in well-controlled laminar fluids with external actuators has led to an extensive number of applications such as: sorting cancer cells ${ }^{15}$ or catalyst particles. 16 However, there is no experimental proof of sorting of microstructures based on the deflection caused by the rotational force.

A rotating object moving relative and inside a fluid experiences the Magnus force. This phenomenon can be observed in sports such as football, 17 golf and tennis, or even at planetary range, ${ }^{18}$ when a sphere or cylinder rotates through fluid, changing its path perpendicular to the sphere's translational direction. Many studies have shown the existence of this phenomenon, 12,19,20 however not with microparticles and at low Reynolds numbers. This work aims at studying the deflection of microparticles $90 \mu \mathrm{m}$ in diameter caused by the Magnus force. 


\subsection{Theory}

The Magnus force relates to the lift force perpendicular to the path of a rotating object flowing in a fluid and is caused by the non-symmetrical flow pattern induced by the spinning object, see Figure 4.1.19,21 When a particle rotates in a fluid, the fluid close to the particle is dragged differently in both parts of the particle. On the left side in Figure 4.1, the fluid is dragged along and pushed to the right, thereby, due to Newton's $3^{\text {rd }}$ law, pushing the particle to the left. The Magnus force has a larger effect at higher Reynolds number (Re) due to the increased inertial forces and therefore acceleration of the fluid producing differences in flow pattern. ${ }^{22-24}$ First, I. Newton and, two centuries later H.G. Magnus discovered and studied this effect on rotating objects. ${ }^{25}$ Many studies have been performed in order to quantify this force ${ }^{26}$ where Rubinov and $\operatorname{Keller}^{27}$ derived the magnitude of the force for low Reynolds number as,

$$
\boldsymbol{F}_{\text {magnus }}=\pi \rho_{\mathrm{f}} r^{3} \boldsymbol{\Omega} \times \boldsymbol{u},
$$

where $\rho_{\mathrm{f}}\left[\mathrm{kg} / \mathrm{m}^{3}\right]$ is the fluid density, $r[\mathrm{~m}]$ the radius of the particle, $\Omega[\mathrm{rad} / \mathrm{s}]$ the angular velocity and $u_{\|}\left[\mathrm{m} \mathrm{s}^{-1}\right]$ the relative velocity between the particle and the fluid (in the experimental part, we express the rotational velocity in the more intuitive units of revolutions per second [rps]). The force is maximum when the rotation axis is perpendicular to the relative velocity.

Previous work has experimentally demonstrated the existence of the Magnus force at low Re. Oesterlé et al. ${ }^{28}$ used metal spheres few centimetres big attached to a thread in order to spin them to quantify the Magnus force at Re between 10 and 140. Others $18,20,24,29$ studied the phenomena at higher Re, from 300 to $10^{5}$, however, to the best of our knowledge, the study of the Magnus force has never been done at $R_{e} \approx 1$, with micro-particles $90 \mu \mathrm{m}$ in diameter. This could be a consequence of the trouble to work with small particles and the large dependence of the Magnus force on the particle size, see Equation 1, which decreases the deflection considerably when dealing with micro or nanoparticles. ${ }^{30}$ 


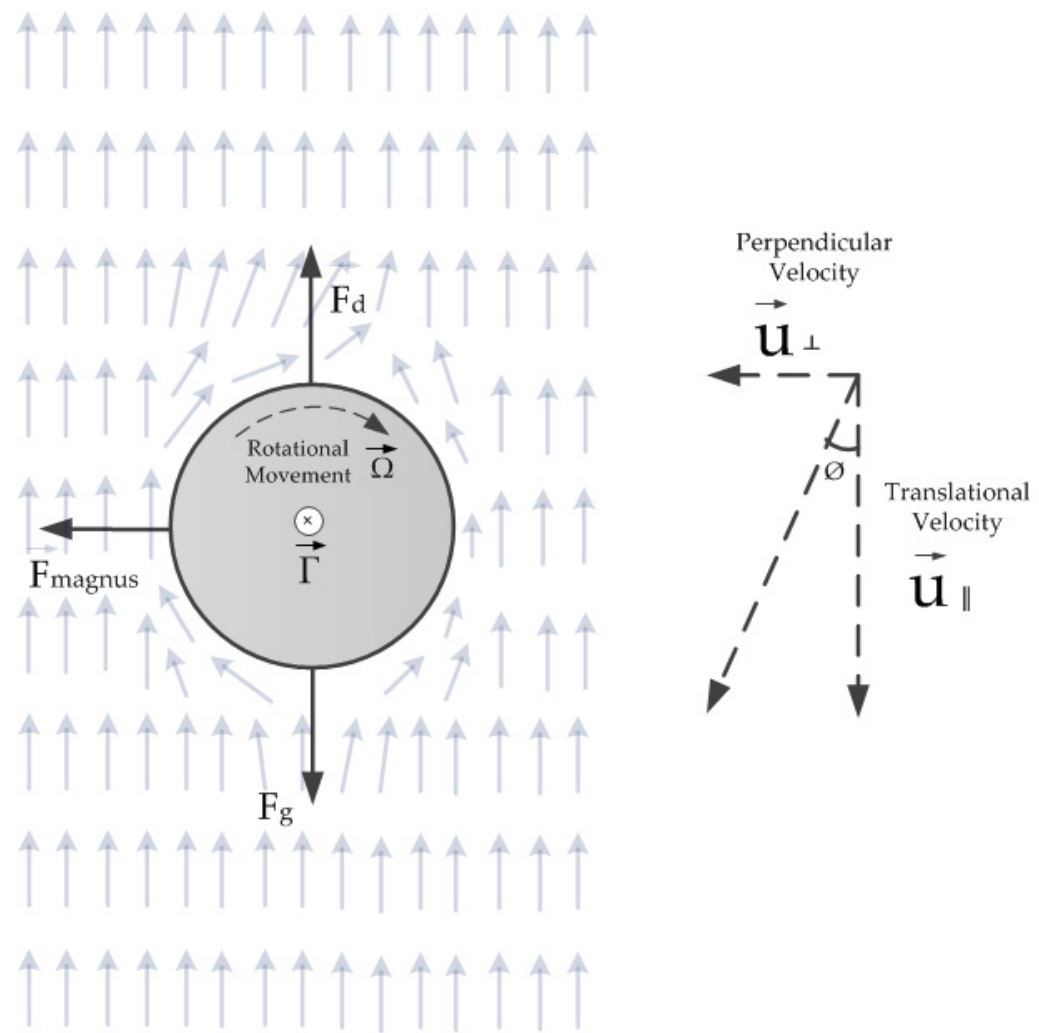

Figure 4.1. Schematic drawing of the principle causing the Magnus force. The fluid on the left part of the particle is dragged along to the right side, thus pushing the particle to the left.

In order to rotate the particles, a rotating magnetic field will be used so that a torque can be applied over a big spatial region. ${ }^{31}$ The Magnus force is proportional to the rotation of the particles, see Equation 4.1. The rotation velocity is equal to the rotation of the magnet only if the particles can follow the rotation. If the magnet rotation speed is too high, the particles only wobble. To estimate the maximum rotation speed, we assume that the particle has a remanent magnetic moment $m_{\mathrm{p}}\left[\mathrm{Am}^{2}\right]$ and that the field $B[\mathrm{~T}]$ is small compared to the saturation magnetization. Under this condition, the maximum torque obtained can be calculated from

$$
\Gamma \leq \boldsymbol{m}_{p} \times \boldsymbol{B}
$$

A sphere with radius $r$ rotating in a fluid with viscosity $\mu_{\mathrm{f}}[\mathrm{Pa} . \mathrm{s}]$ at a rotational velocity $\Omega[\mathrm{rad} / \mathrm{s}]$ experiences a drag torque in the direction opposite to the rotation,

$$
\boldsymbol{\Gamma}_{d}=-8 \pi r^{3} \mu_{\mathrm{f}} \boldsymbol{\Omega}
$$


By balancing the magnetic and rotational drag torque, we obtain the maximum rotational velocity

$$
\mathbf{\Omega} \leq \frac{m_{p} B}{8 \pi r^{3} \mu_{\mathrm{f}}}
$$

Next to being pulled sideways by the Magnus force, the particles are pulled downwards by gravity as,

$$
\boldsymbol{F}_{\mathrm{g}}=\left(\rho_{\mathrm{p}}-\rho_{\mathrm{f}}\right) \boldsymbol{g} V_{\mathrm{p}}
$$

where $\rho_{\mathrm{p}}, \rho_{\mathrm{f}}\left[\mathrm{kg} \mathrm{m}^{-3}\right]$ are the particle and fluid mass density respectively, $g$ [m s $\left.\mathrm{m}^{-2}\right]$ the gravitational acceleration constant and $V_{p}\left[\mathrm{~m}^{3}\right]$ the volume of the particle. A sphere moving in a fluid encounters a translational drag force in the opposite direction to the movement,

$$
\boldsymbol{F}_{\mathrm{d}}=-6 \pi r \mu_{\mathrm{f}} \boldsymbol{u}
$$

Balancing the forces, and considering that the magnetic torque is applied perpendicular to $g$, we obtain the velocity of the particle, which we decompose in the translational and perpendicular components, see Figure 4.1:

$$
\begin{gathered}
\boldsymbol{u}_{\|}=\frac{\left(\rho_{\mathrm{p}}-\rho_{\mathrm{f}}\right) \boldsymbol{g} V_{\mathrm{P}}}{6 \pi \mu_{\mathrm{f}} r}, \\
\boldsymbol{u}_{\perp}=\frac{\rho_{\mathrm{f}} r^{3} \boldsymbol{\Omega} \boldsymbol{u}_{\|}}{6 \pi \mu_{\mathrm{f}} r}
\end{gathered}
$$

The tilt angle of the trajectory is therefore

$$
\phi=\tan ^{-1}\left(\frac{u_{\perp}}{u_{\|}}\right)=\tan ^{-1}\left(\frac{\rho_{\mathrm{f}} r^{2}}{6 \pi \mu_{\mathrm{f}}} \Omega\right)=\tan ^{-1}\left(\frac{r^{2}}{6 \pi v_{\mathrm{f}}} \Omega\right)
$$

When the rotational speed is zero, the particles follow the gravitational force and the tilt angle is zero. The tilt angle increases with increasing sphere radius $r$, and decreases with increasing kinematic fluid viscosity $v_{\mathrm{f}}\left[\mathrm{m}^{2} / \mathrm{s}\right]$.

We cannot avoid some inhomogeneity in the magnetic field. Therefore, the particles will experience a magnetic force along the magnetic field lines,

$$
\boldsymbol{F}_{\mathrm{m}}=m_{\mathrm{p}} \nabla B
$$




\subsection{Materials and methods}

There are three requirements that the fluidic system must accomplish. First, due to the small deviations expected by the Magnus force and in order to facilitate the resulting trajectory measurement, the particles should start at very similar positions in order to experience similar magnetic forces. Second, the system should be long enough to track long trajectories and third, the particles should be clearly seen through the fluidic system walls. Figure 4.2 shows the 3-D printed fluidic system with 3 inlets and 1 outlet. 2 of the 3 inlets are used to introduce the liquid and the $3^{\text {rd }}$ inlet is used to introduce the magnetic particles, also see Figure B.1a in Appendix. The chamber is $7 \mathrm{~cm}$ long and $0.5 \mathrm{~cm}$ wide and deep. To maximize the deflection caused by the Magnus force, the time of the particles inside the chamber should be maximized. Therefore, the system will be used at no-flow conditions, letting the particles sink from the inlet to the outlet of the chamber. Normally, liquid flow, or magnetic, electric or acoustic fields are used to focus the particles inside a microfluidic channel. However, due to the no-flow conditions and the trouble of introducing actuators inside the 3-D printed chip, a new method to focus the particles at the same position was developed. Figure 4.2c shows a zoom-in of the particle's inlet of the microfluidic chip where a zig-zag inlet can be observed. As can be seen in Figure B.3 in Appendix, particles were rolling downwards and therefore entering the big chamber at the same position. Although some of the particles started at very similar positions, this varied due to its large sensitivity on other factors such as: the amount of particles arriving to the big chamber at the same time and disturbing each other or by any small flow perturbation caused by the pipetting of the particles inside the inlet.

In order to observe the particles and seal the chamber, a glass slide was glued on the front part of the chip, see Figure B.1a in Appendix. 


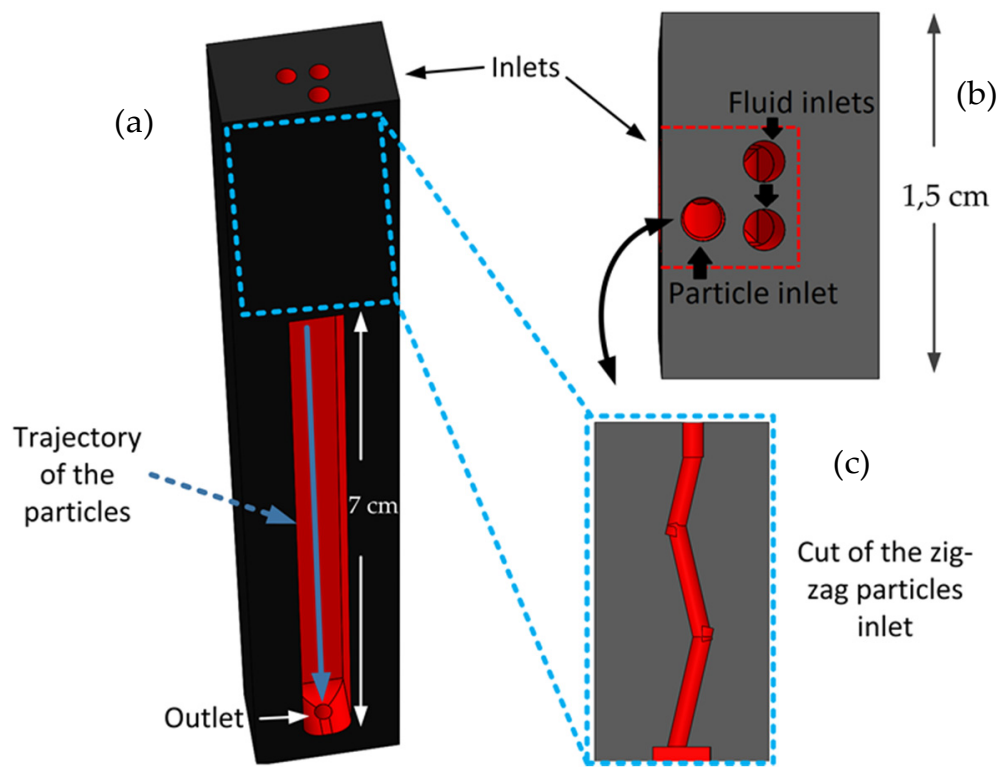

Figure 4.2. (a) Schematic drawing of the fluidic chip showing the 3 inlets and chamber where the particles rotate, (b) the 3 inlets and (c) zoom-int of the zig-zag particles inlet.

In order to rotate the particles and avoid any attraction to the magnets, the magnetic field that is used should be as strong and homogeneous as possible. Therefore, bigger magnets providing less gradient over a particle are better than smaller magnets. Also, by placing another magnet on the opposite side of the fluidic chip the gradient, and therefore its magnetic force towards the magnets, will be reduced, see Figure 4.3. In order to rotate the particles, the magnets were placed with the same poles facing each other and rotating around the $y$-axis, see Figure 4.3. 8 magnets $1 \mathrm{~cm}$ wide, $4 \mathrm{~cm}$ deep and $1 \mathrm{~cm}$ long were purchased from Supermagnete. Each rotating arm consisted of 4 magnets. In order to observe the particle's trajectory a silicon wafer acting as a mirror was glued at a $45^{\circ}$ angle to the fluidic system's wall, see Figure B.1a in in Appendix.

Both magnets should rotate at the same speed in order to avoid magnetic field distortion, but due to the fluidic connections and introduction of the particles both magnets couldn't be attached together. Therefore, a new mechanical system was developed to rotate both magnets at the same speed. Figure 4.4a shows the system consisting of an electrical motor and a few gears that are connected together to rotate the magnets at the same speed, see Figure B.1b and c. in Appendix. Also, for security reasons, a new system was developed to mount the magnets in the rotating arms. As can be seen in Figure $4.4 \mathrm{~b}$, first the magnets are stuck together with a separator in the middle and subsequently screwed in one of the rotator's arms. Thereafter, the other rotating arm is approached and screwed to the second magnet's support. Last, both magnet supports are separated. The magnets repel each other due to their 
configuration therefore the second arm is pushed away by the magnets. The separation of the magnets can be adjusted with extra screws in the rotating arms.

The rotating speed of the magnets was adjusted with a Crouzet DC motor model 820580002 controlled by a Conrad PS 405 Pro power supply. A Grasshopper3 GS3-U3-23S6M high-speed camera was used to observe and record the trajectories of the particles. The 3-D printed chip was designed in SolidWorks and printed with a Formlabs Form 2 printer.

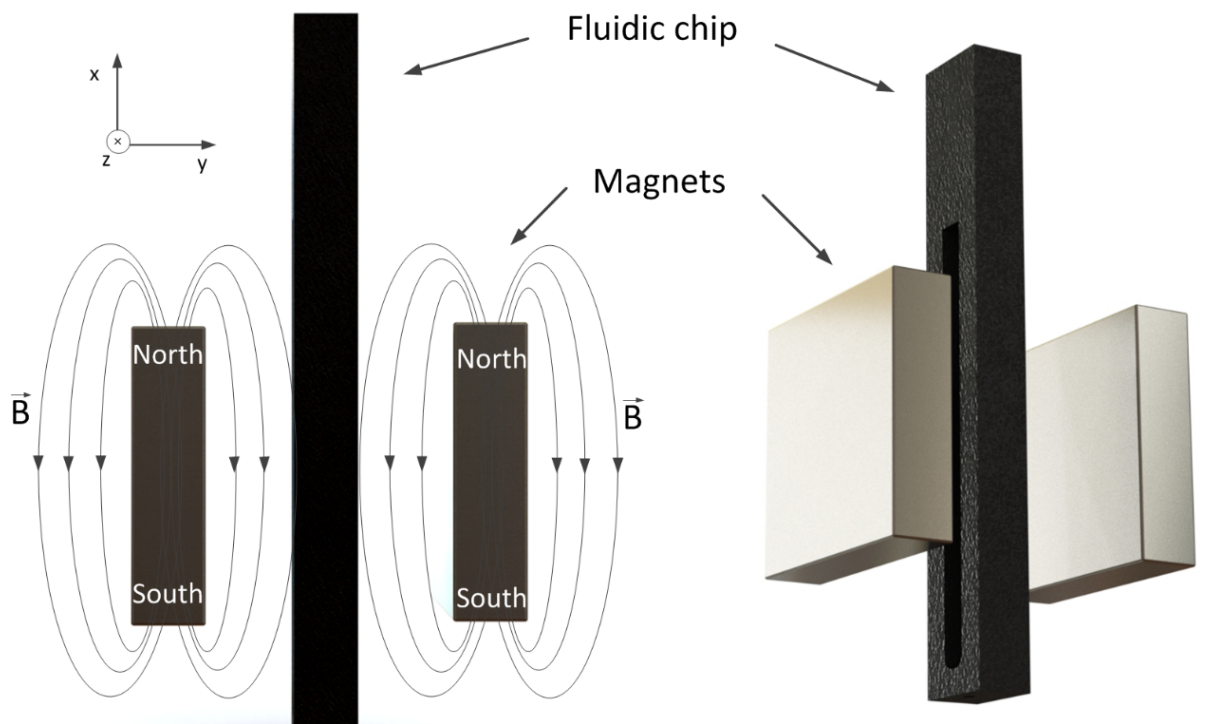

Figure 4.3. (a) Schematic side-view drawing and magnetic field distribution used to rotate the janus particles inside the fluidic chip. (b) Diagonal-view of the magnets configuration.

The particles used in this study are magnetic Janus particles 70-90 $\mu \mathrm{m}$ in diameter and purchased from Cospheric. Their core is made of borosilicate and they are half coated with a superparamagnetic material.

Some Janus particles were placed between two $5 \times 5 \mathrm{~mm}$ tape layers and introduced in a Quantum Design PPMS-VSM (Physical property measurement system vibrating sample magnetometer) to measure their magnetic moment. The scan rate was $1.5\left[\mathrm{mT} \mathrm{s}^{-1}\right]$ from $5 \mathrm{~T}$ to $-5 \mathrm{~T}$ and up to $5 \mathrm{~T}$ again at room temperature. Figure B.2 in in Appendix presents the experimentally obtained magnetization of the janus particles. From the magnetization curve, it can be concluded that the janus particles have hysteresis and that their magnetic moment saturates at $\approx 0.5 \mathrm{~T}$ having a susceptibility of $610^{-11}\left[\mathrm{Am}^{2} \mathrm{~T}^{-1}\right]$ and a remanent moment $m_{p}$ is $1.510^{-11}\left[\mathrm{Am}^{2}\right]$ per particle. By calculating the magnetic field using the software Cades in z-direction, which is the same direction as the magnus force, and using Equation 4.4, the magnetic force per particle can be estimated resulting in average in $0.27 \mathrm{pN}$. This is and order of magnitude lower than the calculated magnus force 
$6.25 \mathrm{pN}$ (taking DI water with $\mu_{f}=1 \mathrm{mPa} . \mathrm{s}$ and $5 \mathrm{rps}$ as the rotational speed). It is worth mentioning that the magnetic force is linearly dependent to the $z$-axis, being 0 at the centre of both magnets $(x, y, z)=(0,0,0)$ and increasing until $2.2 \mathrm{pN}$ at $(x, y, z)=(0,0,5) \mathrm{mm}$. Although both $z$-direction forces are similar, the trajectories of the particles are dominated by the gravity force which is 3 orders of magnitude larger, in the $\mathrm{nN}$ range.

2 solutions were used, DI water and a mix of glycerol and water in a 1:3 volume ratio. Three pipette tips were glued in the 3 inlets to facilitate the introduction of liquids and particles. The chip was filled with a pipette until the liquid reached the top of the pipette tips. The characterization of the rotation of the system was performed using a Helio-Strob micro2. Matlab was used to track the particles trajectories and Cades was used to simulate the magnetic field and magnetic force.

3 different experiments were performed where just the liquid viscosity and the rotation speed of the particles were modified. The experimental procedure consisted of different steps. First, the rotation of the magnets was set to a given rotational speed. Second, the janus particles were introduced in the fluidic chip via a pipette. Thereafter, they rolled and fell into the main channel. Third, their trajectories over $1 \mathrm{~cm}$ length inside the fluidic system were recorded and subsequently analysed. 6 different steps in each experiment were performed, three clockwise and three anti-clockwise. The centre of the trajectories were manually centred to 0 in order to visualize the difference between experiments.

\subsection{Results and discussion}

Figure 4.5 shows the experimentally obtained angles of the trajectories of the particles and their cumulative distribution function (CDF) assuming normal distributions. In Figure 4.5a a clear difference can be observed between both rotating directions using DI water, $\left(v_{f}=1.010^{-6}\left[\mathrm{~m}^{2} / \mathrm{s}\right]\right)$ and $5[\mathrm{rps}]$ as rotational speed. The perpendicular direction is in agreement with theory, particles rotating clockwise moved to the left and particles rotating anticlockwise moved to the right. The experimental difference in angle means between particles rotating in both directions was $1.15 \pm 0.14\left[^{\circ}\right]$ however, the calculated angle from Equation 4.9 resulted in $0.39\left[^{\circ}\right]$. It is expected that the deflection is increased by the particles attraction to the corners of the magnets hence, any small difference caused by the rotation of the particles may be increased by the magnetic force. Figure $4.5 \mathrm{~b}$ shows the same experiment but using a lower rotational speed, 2 [rps] and, as expected by Equation 4.9, the angle difference becomes smaller than that in the previous experiment, being both the experimental and theoretical angles obtained, $0.42 \pm 0.15\left[^{\circ}\right]$ and 0.15 $\left.{ }^{\circ}\right]$ respectively. Last, Figure $4.5 \mathrm{c}$ presents the experimental angles obtained using a higher viscosity liquid, $\left(v=2.310^{-6}\left[\mathrm{~m}^{2} / \mathrm{s}\right]\right)$ and lower rotational speed, 2 [rps], showing no difference between particles rotating in both directions 
confirming that an increase in viscosity reduces the deflection due to the rotation of particles.

(a)
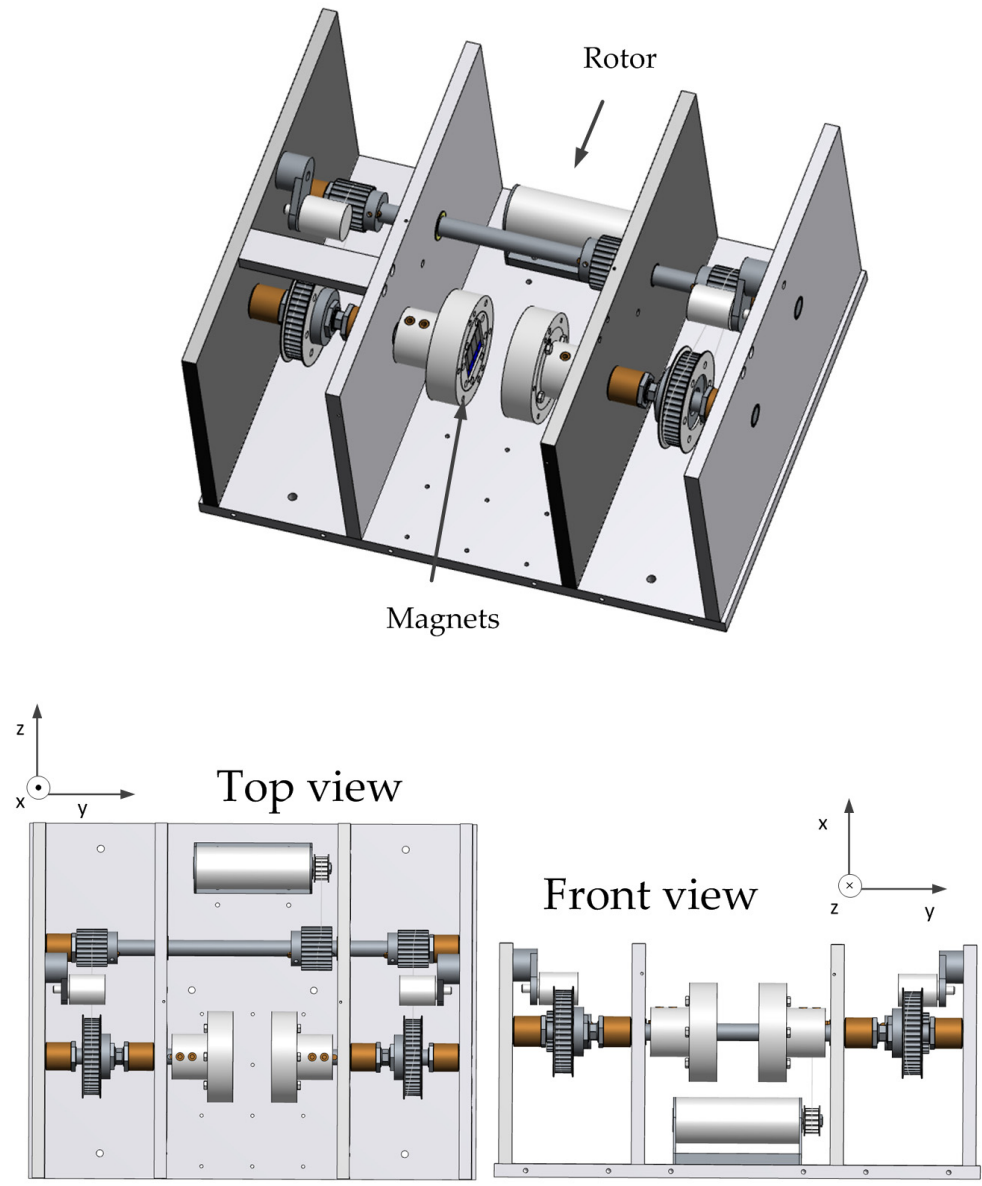

(b)
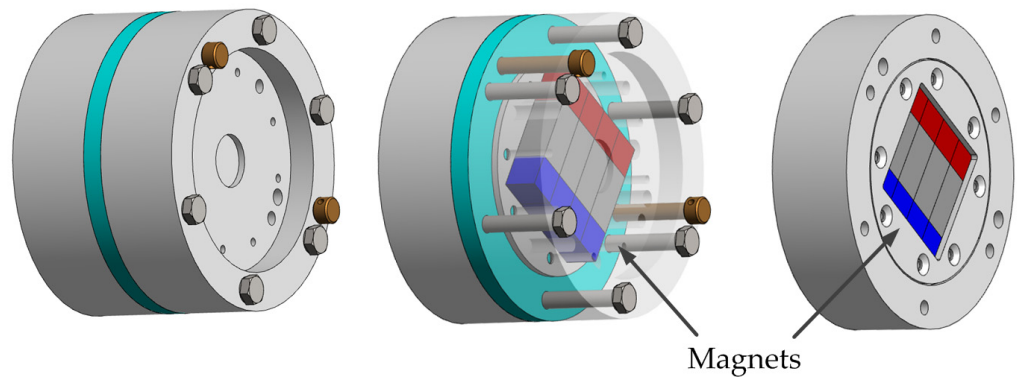

Figure 4.4. Solidworks drawing of the (a) rotating magnetic field setup where the electric motor rotates the magnets at the same rotational velocity and (b) the mounting of magnets setup. 
An experiment with a high viscosity $\left(v=2.310^{-6}\left[\mathrm{~m}^{2} / \mathrm{s}\right]\right)$, and high rotational speed 5 [rps], was also performed, however most of the particles weren't able to follow the rotating magnetic field due to the higher viscosity. Assuming the magnetic field to be in the order of $90[\mathrm{mT}]$, the maximum rotation velocity in the high viscosity medium ( $\mu=2.3$ [mPas]) is, according to Equation 4.4, in the order of 40 [rps]. Therefore we expected the particles to rotate. In addition, it is worth mentioning that the final deflection difference between particles rotating in both directions in Figure 4.5 a was less than $\approx 500$ $[\mu \mathrm{m}]$ over a translational distance of $\approx 1[\mathrm{~cm}]$. This confirms the relatively small deflections expected due to the rotation of the particles, however further improvements could be done in order to enhance the deflection difference such as, increasing the magnetic field strength inside the fluidic system by placing the magnets closer together which would allow a faster rotation of the particles.

\subsection{Conclusions}

In conclusion, we dropped magnetic Janus particles $90 \mu \mathrm{m}$ in diameter in a liquid in the presence of a rotating magnetic field. The particles rotated with the field and are therefore subject to a Magnus force that caused a measurable tilt of up to $1.15\left[^{\circ}\right]$ in their trajectories. The direction of the tilt agrees with the theory. The tilt angle increased with increasing rotation velocity and decreasing viscosity. The particles no longer followed the magnetic field in a medium with a kinematic viscosity of $2.310^{-6}\left[\mathrm{~m}^{2} / \mathrm{s}\right]$ at a rotation velocity of 5 [rps], and therefore no longer showed a tilt. This effect allows us to separate particles based on their magnetic moment, which is of great interest for the analysis of magnetic microparticles such as catalyst particles or cells labelled with magnetic materials. 
(a)

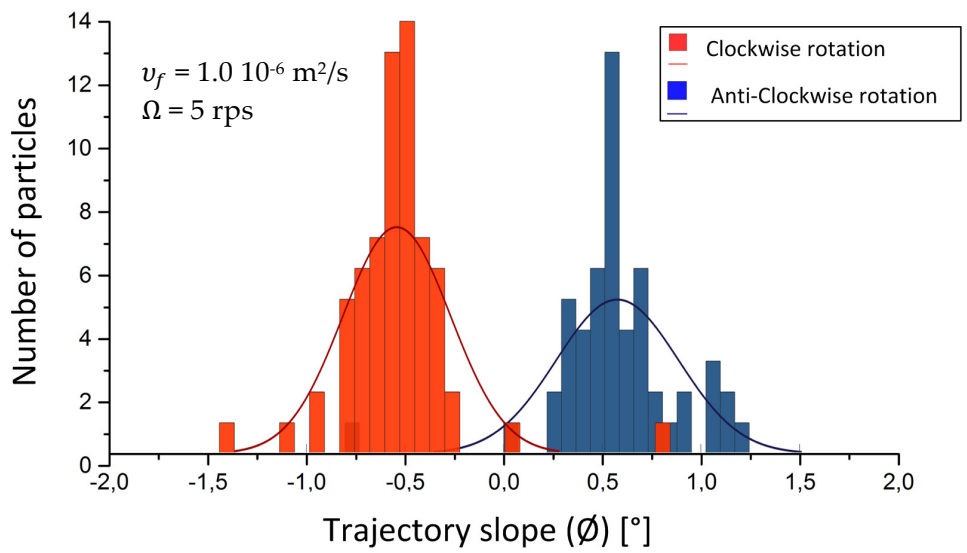

(b)

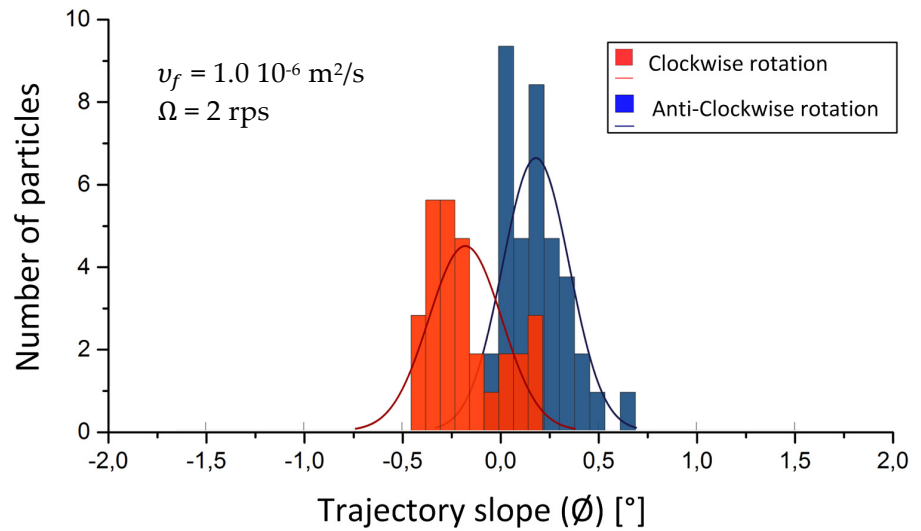

(c)

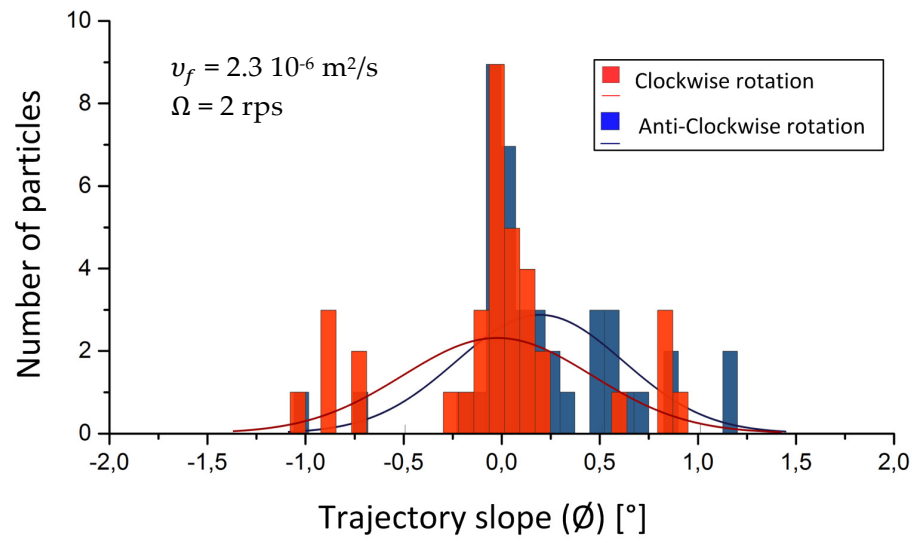

Figure 4.5. Histograms and their cumulative distribution function (CDF) of the trajectory slope angles in z-direction of particles rotating clockwise (red) and anticlockwise (blue) (a) with low viscosity (water) and $5 \mathrm{rps}$, (b) low viscosity (water) and $2 \mathrm{rps}$ and (c) high viscosity (mix of water and glycerol 3/1 v/v) and 2 rps. 


\subsection{References}

1. Vogt, E. T. C. \& Weckhuysen, B. M. Fluid catalytic cracking: recent developments on the grand old lady of zeolite catalysis. Chem. Soc. Rev. 44, 7342-7370 (2015).

2. Meirer, F. et al. Mapping metals incorporation of a whole single catalyst particle using element specific X-ray nanotomography. J. Am. Chem. Soc. 137, 102-105 (2015).

3. Meirer, F. et al. Life and death of a single catalytic cracking particle. Sci. Adv. 1, e1400199 (2015).

4. Liu, Y., Meirer, F., Krest, C. M., Webb, S. \& Weckhuysen, B. M. Relating structure and composition with accessibility of a single catalyst particle using correlative 3-dimensional micro-spectroscopy. Nat. Commun. 7:12634, (2016).

5. Solsona, M. et al. Magnetophoretic Sorting of Single Catalyst Particles. Angew. Chemie Int. Ed. 57, 10589 -10594 (2018).

6. Stratton, J. A. Electromagnetic Theory. (McGraw-Hil, 2011).

7. Whitesides, G. M. The origins and the future of microfluidics. Nature 442, 368-373 (2006).

8. Gossett, D. R. et al. Label-free cell separation and sorting in microfluidic systems. Anal. Bioanal. Chem. 397, 3249-3267 (2010).

9. Amini, H., Lee, W. \& Di Carlo, D. Inertial microfluidic physics. Lab Chip 14, 2739-2761 (2014).

10. Wyatt Shields IV, C., Reyes, C. D. \& López, G. P. Microfluidic cell sorting: a review of the advances in the separation of cells from debulking to rare cell isolation. Lab Chip 15, 1230-1249 (2015).

11. Sajeesh, P. \& Kumar Sen, A. Particle separation and sorting in microfluidic devices : a review. 1-52 (2014).

12. Saffman, P. G. The lift on a s $m$ a 11 sphere in a slow shear flow. 22, (1965).

13. Svahn, H. A. \& Van Den Berg, A. Single cells or large populations? Lab Chip 7, 544-546 (2007).

14. Zhang, J. et al. Fundamentals and applications of inertial microfluidics: A review. Lab Chip 16, 10-34 (2016).

15. Li, P.et al. Acoustic separation of circulating tumor cells. Proc. Natl. Acad. Sci. 112, 4970-4975 (2015).

16. Solsona, M. et al. Magnetophoretic Sorting of Single Catalyst Particles. Angew. Chemie - Int. Ed. 57, 10589-10594 (2018).

17. Kensrud, J. R. Determining Aerodynamic Properties of Sports Balls in SITU. (2010).

18. Forbes, J. C. Curveballs in protoplanetary discs: The effect of the Magnus force on planet formation. Mon. Not. R. Astron. Soc. 453, 1779-1792 (2015).

19. Barkla, H. M. \& Auchterlonie, L. J. The Magnus or Robins effect on rotating spheres. J. Fluid Mech. 47, 437-447 (1971). 
20. Rem, P. C., Fraunholcz, N. \& Schokker, E. A. Magnus separation. Sep. Sci. Technol. 37, 3647-3660 (2002).

21. Seifert, J. A review of the Magnus effect in aeronautics. Prog. Aerosp. Sci. 55, 17-45 (2012).

22. Bagchi, P. \& Balachandar, S. Effect of free rotation on the motion of a solid sphere in linear shear flow at moderate Re. Phys. Fluids 14, 27192737 (2002).

23. Kray, T., Franke, J. \& Frank, W. Magnus effect on a rotating sphere at high Reynolds numbers. J. Wind Eng. Ind. Aerodyn. 110, 1-9 (2012).

24. Kray, T., Franke, J. \& Frank, W. Positive and negative Magnus effect on a rotating soccer ball at high Reynolds numbers. 6th Eur. African Conf. Wind Eng. EACWE 2013 1-8 (2013).

25. Magnus, G. liber die Abweichung der Geschlosse, und eine auffallende Erscheinung bei rotierenden Korpern. Poggendorfs Ann. der Phys. und Chemie, 88, 1 (1853).

26. Beck, D. Magnus force acting upon a rotating sphere passing in an incompressible viscous flow. (Charles University, 2018).

27. Rubinow, S. I. \& Keller, J. B. The transverse force on a spinning sphere moving in a viscous fluid. J. Fluid Mech. 11, 447-459 (1961).

28. Oesterl\&\#x000E9; B. \& Dinh, T. B. Experiments on the lift of a spinning sphere in a range of intermediate Reynolds numbers. Exp. Fluids 25, 1622 (1998).

29. Tsuji, Y., Morikawa, Y. \& Mizuno, O. Experimental Measurement of the Magnus Force on a Rotating Sphere at Low Reynolds Numbers. J. Fluids Eng. 107, 484 (1985).

30. Denisov, S. I., Lyutyy, T. V., Reva, V. V. \& Yermolenko, A. S. Temperature effects on drift of suspended single-domain particles induced by the Magnus force. Phys. Rev. E 97, 32608 (2018).

31. Moerland, C. P., Ijzendoorn, L. J. Van \& Prins, M. W. J. Rotating magnetic particles for Lab on a Chip applications - a comprehensive review. Lab Chip 19, 919-933 (2019). 


\section{Chapter

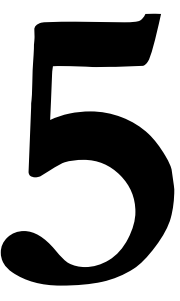

\section{Analysis of Catalyst Particle Activity by means of Impedance Spectroscopy}

Catalyst particle characterization is normally performed in packed bed reactors where thousands of particles are analysed at the same time. The obtained ensemble characteristics may have a wide dispersion. Therefore, single particle characterization may be useful, but normally requires expensive technology. Hence inexpensive methods to analyse single catalyst particles are needed. Fluid catalytic cracking particles are catalysts that deactivate due to metals that accumulate in them and change their dielectric properties. Here we report a microfluidic chip that measures the dielectric properties of fluid catalytic cracking particles using flow impedance spectroscopy. The catalyst particles were sorted by their metal content and their dielectric properties were measured. Unfortunately, no difference in impedance was observed between particles with different metal content.

\section{This chapter is based on:}

Solsona, M., Hendrix, J., Odijk, M., Olthuis, W., van den Berg, A. Impedance-based Microfluidic Particle characterization system with Atto-Farad Sensitivity. In preparation. 


\subsection{Introduction}

Catalyst particles are normally synthesized in reactors which lack of control over individual particle composition, resulting in intra and inter-particle heterogeneities. Activity characterization of catalyst particles is normally performed in large vessels providing an ensemble average which masks the individual role of each particle. Therefore, new methods to measure the activity of single catalyst particles are needed.

Fluid catalytic cracking (FCC) particles are porous catalysts responsible for cracking long chain hydrocarbons into smaller and more useful molecules. ${ }^{1}$ However, during the cracking process, they deactivate due to, but no limited to metals such as: Fe, $\mathrm{Ni}$ and $\mathrm{V}$ coming from the feedstock. In order to maintain the efficiency in the reactor, FCC particles are constantly replaced by fresh ones resulting in a mixture called Equilibrium catalyst (ECAT FCC). The particles density slowly increases during the process, therefore giving an indication of their activity. ${ }^{1-4}$ The increase in particles density can be analysed using the sinkfloat density separation method, which sorts particles using liquids with different densities. However, this method is performed in a discontinuous manner which doesn't allow for high throughput analysis. ${ }^{3}$ As shown in Chapter 3, ECAT FCC particles can also be sorted based on activity using magnetic fields. This method resulted in an efficient way to sort ECAT FCC particles by Fe content, however the rest of the metals was not taken into account. Besides density, the dielectric properties of the ECAT FCC particles also change when metals accumulate in them. The dielectric constant and conductivity of the deactivating metals and metal oxides are both greater than those of the main active materials of the FCC particles, silica and alumina. Therefore, measuring the dielectric properties of ECAT FCC particles could be a method to analyse their activity.

Flow impedance cytometry (FIC) is a non-invasive technique that measures the dielectric properties of cells at high-throughput. It is achieved by applying an alternating voltage and measuring the corresponding current on an array of electrodes in a fluidic channel. ${ }^{5-10}$ FIC has been widely used to measure the dielectric properties of cells but, to the best of our knowledge, it has never been used to measure the dielectric properties of catalyst particles.

When measuring very small signals, FIC normally requires extra systems to improve the quality of the signal, such as a lock-in amplifier. This device is able to detect very small AC signals, even when the noise that is present is several orders of magnitude higher than the signal itself. ${ }^{11-13}$ This is possible because a lock-in amplifier is able to measure at a very narrow frequency bandwidth, which can be determined by the user. The basic building blocks of a lock-in amplifier are shown in Figure 5.1. 


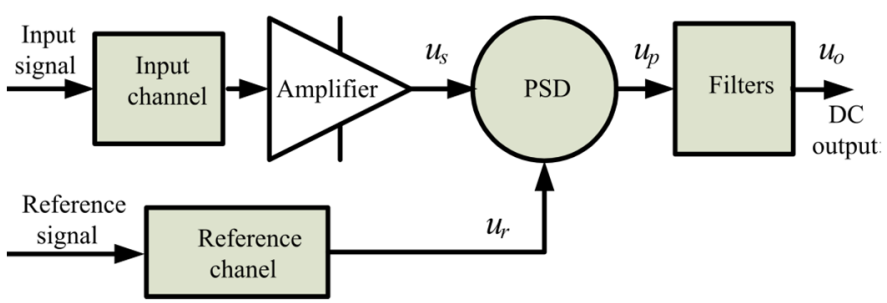

Figure 5.1. Block diagram showing the basic elements of a lock-in amplifier such as: input channel, amplifier, phase-sensitive detector (PSD), filters and reference channel. (adapted from ${ }^{4}$.)

After amplification, the signal is multiplied in a phase-sensitive detector with a reference signal which has a predetermined frequency. The basic working principle of a lock-in amplifier is based on multiplication of two sinusoidal functions which is as follows:

$$
\begin{gathered}
A \sin \left(\omega_{1} t\right) \cdot B \sin \left(\omega_{2} t\right)= \\
=\frac{1}{2} A B\left(\cos \left(t\left(\omega_{1}+\omega_{2}\right)\right)-\left(\cos \left(t\left(\omega_{1}-\omega_{2}\right)\right)\right),\right.
\end{gathered}
$$

where $\omega_{1}$ and $\omega_{2}$ are the frequencies of the two signals in [ $\mathrm{rad} \mathrm{s}^{-1}$ ] and $A$ and $B$ the amplitudes of the signals [V] discarding any phase differences. When the reference frequency is chosen such that frequencies $\omega_{1}$ and $\omega_{2}$ are exactly equal, the result of the multiplication is a combination of a DC signal and a signal with twice the frequency. After using a simple low-pass filter, only the DC element will remain. The noise which is present, is a combination of $1 / \mathrm{f}$ noise and white noise and will therefore mostly be present at low-frequencies. ${ }^{14}$ This noise is also multiplied by the reference signal, which will not result in a DC signal for all frequencies which are not equal to the reference signal. After low-pass filtering, most of the noise will therefore be removed and only the noise which has a frequency very close to the reference signal will thus remain.

The aforementioned method allows measuring small differences in dielectric properties. However, the impedance measured by our system also depends on the size of the particles, therefore their size should also be accounted for a better signal interpretation. For this, opacity can be used. This method measures the impedance at two different frequencies. At low and high frequencies, the resistance and the capacitance of the particles can be measured, both of which depend on the particle size. When dividing the high by the low frequency impedance, the result becomes independent of the size of the particle and therefore just dependent on the material properties. ${ }^{15}$

In this chapter, the dielectric properties of the ECAT FCC particles will be measured by means of microfluidic impedance spectroscopy, opacity and a lock-in amplifier. A microfluidic chip will be fabricated and its sensitivity will be experimentally verified with polystyrene particles and anisole. The 
combination of these 2 materials allows to test the system at very small signals and still visually detecting the particles' size. Last, the dielectric properties of the ECAT FCC particles will be measured.

\subsection{Materials and methods}

Two electrolytes were used, a $1 \mathrm{mM}$ PBS solution and anisole as both purchased from Sigma Aldrich. Two types of particles were used: polystyrene particles and ECAT FCC particles. Polystyrene particles were obtained from Microspheres-Nanospheres. ECAT FCC particles were sorted by the sink-float method into 3 categories: low metal loading (LML), middle metal loading (MML) and high metal loading (HML). ${ }^{16}$ In the experiments, only the LML and HML particles were used.

The signal generation and the lock-in amplifier functionality were performed by the HF2IS Impedance Spectroscope. The impedance spectroscope from Zurich Instruments is capable of generating two anti-phase signals, while simultaneously measuring the system output with high accuracy. A NEMESYS syringe pump from Cetoni $\mathrm{GmbH}$ was used to flow the electrolytes inside the chip. The Delta electronics Dual power supply E018-0.6D was used as power supply. For the video capture, the point grey grashopper 3 and the flycapture 2 software were used. The microfluidic chip was placed in a side-connect chipholder designed by Micronit. Data analysis was done using Matlab and ImageJ.

The glass/SU8/glass microfluidic chip was fabricated using standard photolithoghy techniques, see the masks in section C.4 in Appendix. A detailed process flow is included in section C.5 in Appendix. In short, the electrodes were patterned on Mempax wafers by lift-off. In order to form the channels and bond the wafers, 2 layers of SU8 were used. First, a thick layer of $190 \mu \mathrm{m}$ was deposited and pre-baked. Thereafter, a layer of approximately $30 \mu \mathrm{m}$ was deposited on top of the first one. This "two layers" technique allows for a more smooth surface and therefore a better bonding. Bonding was performed at $120^{\circ} \mathrm{C}$ and $4000 \mathrm{~N}$ for 20 minutes. Thereafter, the chips were diced. Figure 5.2 shows the microfluidic chip. 


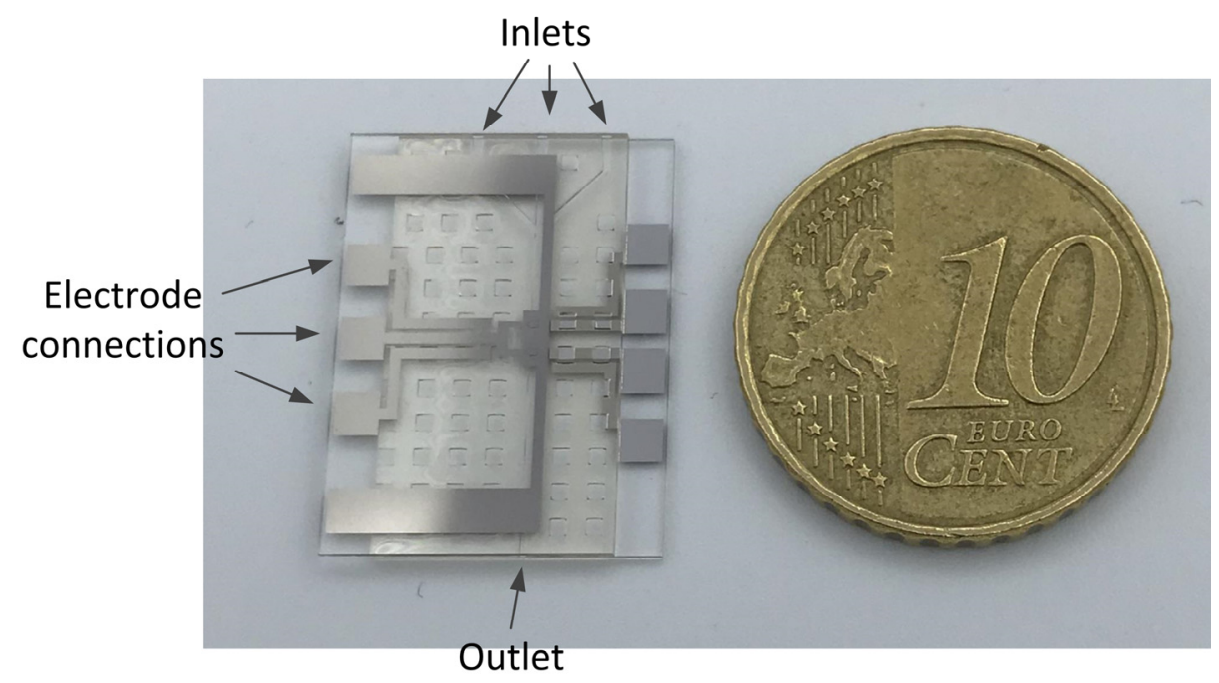

Figure 5.2. Image of the microfluidic chip fabricated to measure the activity of FCC particle.

\subsubsection{Design of the Microfluidic chip}

A simplified drawing of the designed microfluidic chip is shown in Figure 5.3. The depth and width of the electrodes inside the channel were 300 $\mu \mathrm{m}$. The distance between top and bottom electrodes was set at $230 \mu \mathrm{m}$ and the distance between both pairs of electrodes at $1 \mathrm{~mm}$.

Due to the inhomogeneity in the generated electric field, the so-called fringing effect, the final impedance is dependent on the particle's position. To reduce this effect, two extra inlets were added to flow-focus the particles at the same position, however, this will only be applied in one dimension. ${ }^{17}$

As can be seen in Figure 5.3, the chip consisted of 2 sets of 2 electrodes to perform differential measurement. When no particle is located between both sets of electrodes, only the medium will be present. Since both the dimensions and the dielectric properties are identical, the measured impedance difference should be zero. When a particle is present between the electrodes, the impedance of this pair will change due to the dielectric properties of the particle which are different from the medium's. The main advantage of differential measuring is the reduction of common effects. ${ }^{18}$

\subsubsection{Measurement set-up working principle}

To perform differential measurements, both sets of electrodes are driven by two sinusoidal signals which have the same amplitude and frequency but a phase difference of exactly 180 degrees. For the measurements, two frequencies are used for which the resistance and capacitance are dominant respectively. The two sensing electrodes are connected together, therefore current at this node will only be dependent on the difference in the two impedances, Figure 
5.3. The current through this node at low and high frequencies will be defined as,

$$
\begin{gathered}
I_{\text {out }}=I_{\text {sig }}+I_{\text {ref }}=V_{\text {in }}\left(R_{\text {sig }}\right)^{-1}+V_{\text {in }}\left(R_{\text {ref }}\right)^{-1}=V_{\text {in }}\left(\frac{R_{\text {sig }}-R_{\text {ref }}}{R_{\text {sig }} R_{\text {ref }}}\right), \\
I_{\text {out }}=I_{\text {sig }}+I_{\text {ref }}=j \omega V_{\text {in }} C_{\text {sig }}-j \omega V_{\text {in }} C_{r e f}=j \omega V_{\text {in }}\left(C_{\text {ref }}-C_{\text {sig }}\right),
\end{gathered}
$$

respectively, where Iout, $\mathrm{I}_{\text {sig }}$ and $\mathrm{I}_{\mathrm{ref}}[\mathrm{A}]$ are the currents as indicated in Figure 5.3, $\omega$ is the angular frequency $[\mathrm{rad} / \mathrm{s}], \mathrm{C}_{\text {sig }}$ and $\mathrm{C}_{\text {ref }}$ the capacitances of the two electrode pairs [F], Rsig and $R_{\text {ref }}$ the resistances of the two pairs of electrodes [ $\Omega$ ], $\mathrm{V}_{\text {in }}$ is the input voltage [V] and $\mathrm{j}$ the imaginary number. Due to imperfections in the fabrication of the device, the signals sig and ref will not be exactly the same. This will result in an offset of the output current of the microfluidic device which can be adjusted manually.

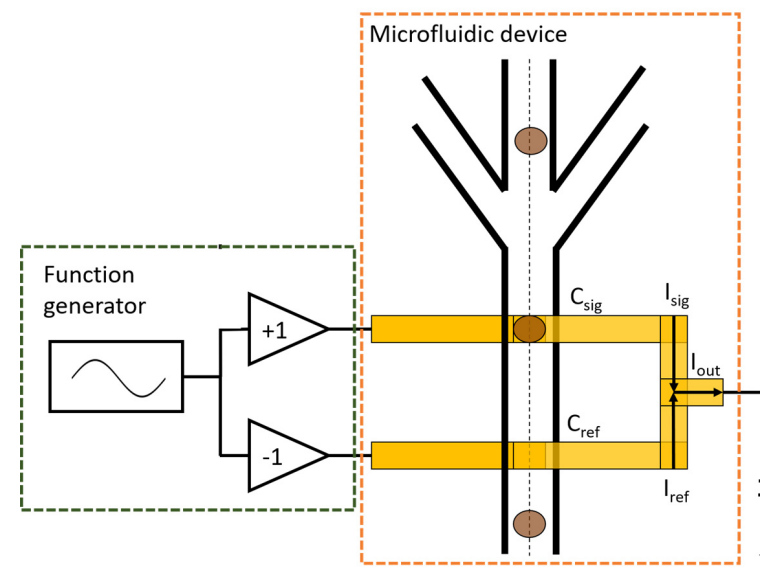

Figure 5.3. Schematic drawing of the microfluidic chip design and signal generator.

Ground electrodes were fabricated around the measuring electrodes in order to shield them. Shielding reduces coupling between the system and noise sources that are present around the setup. The grounded electrodes create a barrier for the electric field lines from the noisy surrounding, see Figure C.1 in Appendix. These field lines will therefore no longer reach the sensitive circuit elements, hereby reducing the amount of noise present in the system. In addition, by adding the grounding planes around the electrodes, the electric field becomes more uniform. As shown in Figure 5.4, the electric field between the electrodes is presented without and with the addition of ground planes respectively. This shows that the uniformity of the electric field around the top measuring electrode is increased by adding ground electrodes. 
In order to measure the capacitance difference of the microfluidic device, the output current of the system should first be converted into a voltage. This is done by using a simple charge amplifier as shown in Figure 5.5. The output of this amplifier is formulated as,

$$
V_{a m p}=-I_{\text {out }} \frac{1}{\frac{1}{R_{a m p}}+j \omega C_{a m p}},
$$

where $V_{a m p}$ is the output voltage of the current amplifier [V], Ramp is the resistance of the amplifier resistor $[\Omega]$ and $C_{a m p}$ the capacitance of the amplifier capacitor [F]. The value of the resistor $\mathrm{R}_{\mathrm{amp}}$ and the capacitance $\mathrm{C}_{\mathrm{amp}}$ are chosen $1 \mathrm{M} \Omega$ and $3 \mathrm{pF}$ respectively, therefore, at relatively high frequencies the amplifying behaviour is dominated by the capacitor. The output of the amplifier then becomes,

$$
V_{a m p}=-I_{\text {out }} \frac{1}{j \omega C_{a m p}} .
$$

Combining Equations 5.2, 5.3 and 5.5 the total expression for the input voltage of the lock-in-amplifier at low and high frequencies is defined as,

$$
\begin{gathered}
V_{a m p \_l o w}=V_{\text {in }} \frac{\left(R_{\text {sig }}-R_{\text {ref }}\right)}{R_{\text {sig }} R_{\text {ref }}}\left(j \omega C_{a m p}\right)^{-1}, \\
V_{\text {amp_high }}=V_{\text {in }} \frac{\left(C_{r e f}-C_{\text {sig }}\right)}{C_{a m p}} .
\end{gathered}
$$

(a)

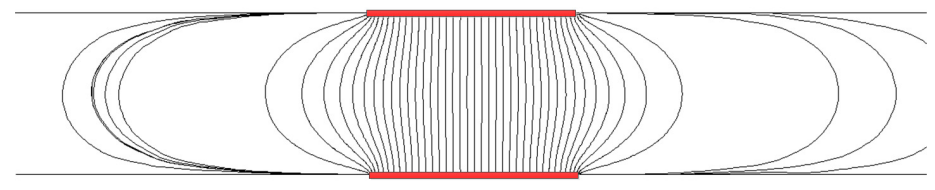

(b)

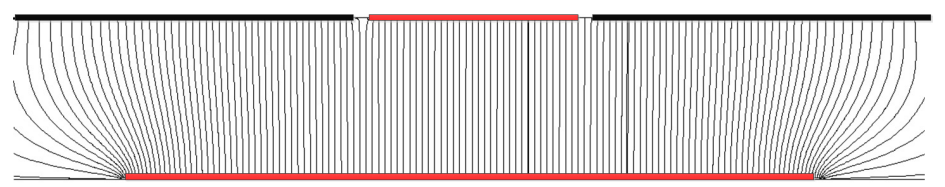

Figure 5.4. 2-D image of a 3-D Comsol simulation of the electric field inside the channel (a) without and $(b)$ with additional ground planes. Here, the top-centre electrode is at (virtual) ground potential. 


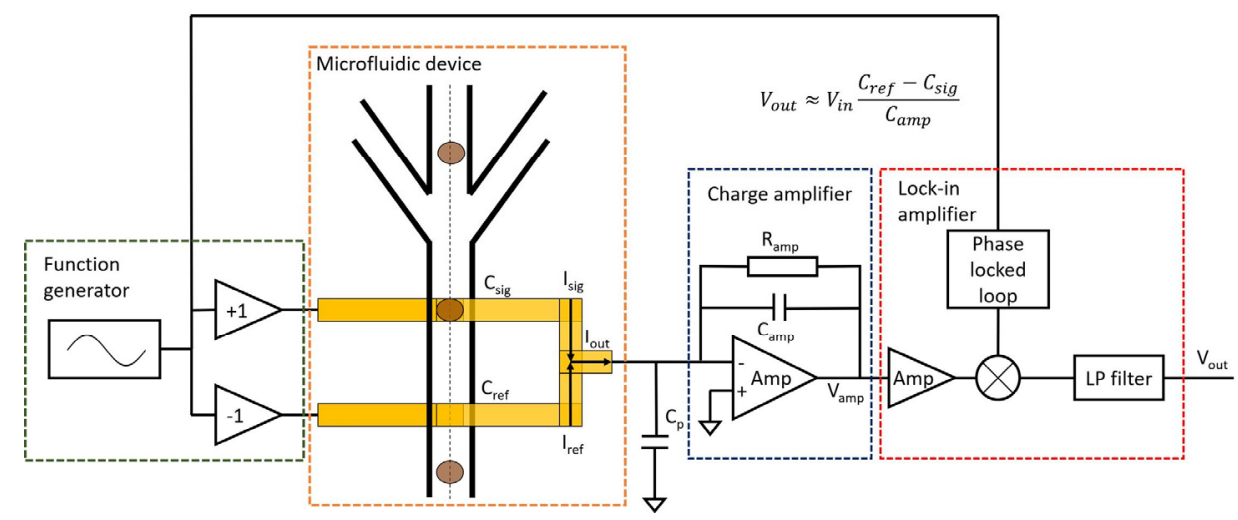

Figure 5.5. Overview of the measurement set-up design using a lock-in architecture.

An overview of the measurement set-up used in the detection of the ECAT FCC particles activity is shown in Figure 5.5. The main settings of the lock-in amplifier are the sample rate and the bandwidth which depend on the velocity of the particles. A lower bandwidth results in a larger reduction of noise. To ensure that the passage of particles are not removed by filtering, a bandwidth of $100 \mathrm{~Hz}$ is used. The sample rate is set at 899 samples per second which is the maximum of the impedance spectroscope.

As explained previously, ground planes were added to the device in order to shield the sensing electrodes from noisy signals around the set-up. For the same reason, all the sensitive elements of the system are placed in a grounded box, see Figure C.2 in Appendix. This box contains two compartments, one for the microfluidic device and another one for the charge amplifier.

\subsection{Results and Discussion}

Figure 5.6 shows typical data at $10 \mathrm{MHz}$ when a PS particle flows in a conductive electrolyte ( $1 \mathrm{mM}$ PBS) between two pairs of parallel electrodes performing differential measurements and, as can be seen, two notable features appear. First, a counter peak (green circle) appears meaning that there is increased conductance due to the PS particles. Second, two small increments in resistance appear in the middle of the larger peak (red circle). In order to clarify these features, a 3-D simulation was performed in Comsol. Figure 5.7 shows the simulated electrical current when a particle flows in the middle and top of the channel, see Figure 5.7a. It is clear that when the particle is at the top of the channel, it causes a very similar trend compared to the experimental results, Figure 5.7b. This is due to the current displacement caused by the particle, highlighted in blue. When no particle is present this current is collected in the ground electrode. However, when a particle is close to the measuring electrode, some extra current ends up in the measurement electrode causing an increase in current, see Figure 5.7c. 


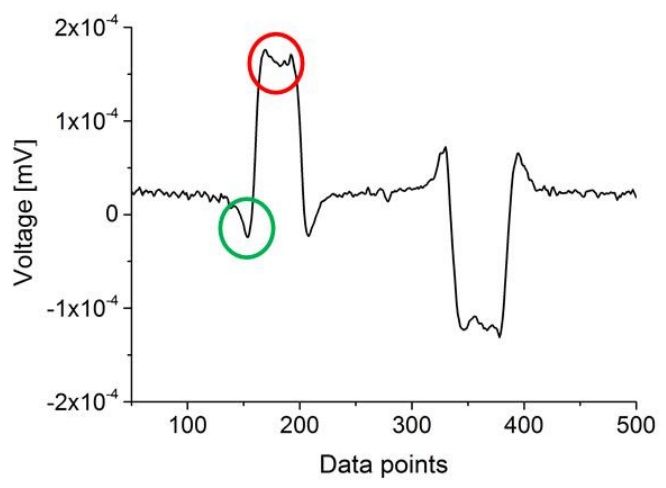

Figure 5.6. Experimental data of a polystyrene particle flowing in the microfluidic chip.

(a)

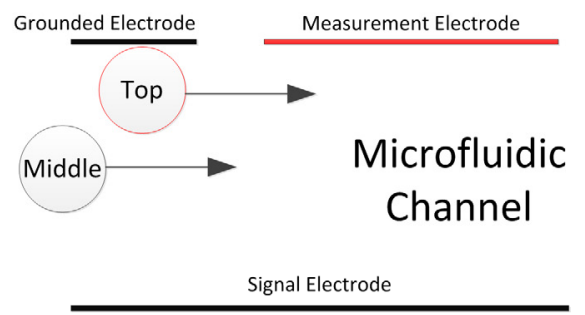

(c)

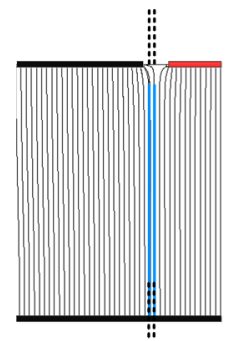

(b)
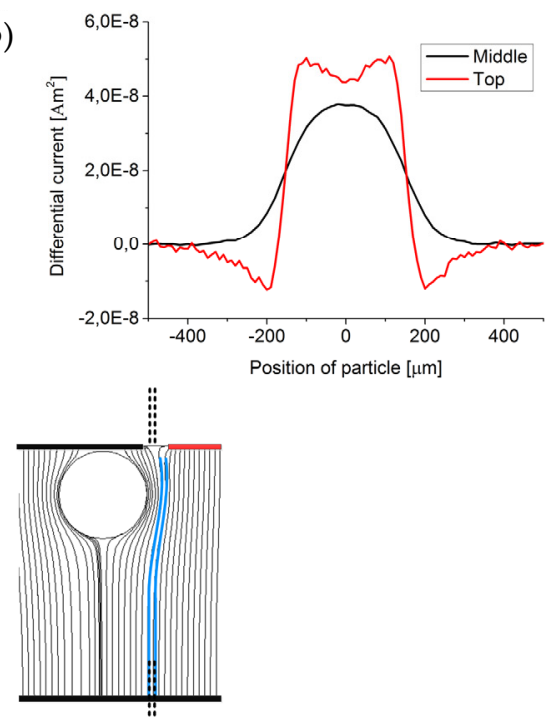

Figure 5.7. (a) Schematic drawing and (b) simulated current difference of two particles flowing at two positions of the microfluidic channel. (c) Simulated distorsion of the electric field caused by a particle flowing at the top part of the microfuidic channel.

Next, the sensitivity of the system at high frequencies was tested with polystyrene particles in anisole. Anisole was used due to its very similar dielectric constant compared to the PS particles allowing to test the system at very small signals while still being able to observe the particles. Figure 5.8 presents the simulation using the homogeneous particle model from Sun et al. ${ }^{21}$, and experimental results showing similar behaviour. As can be observed, the capacitance difference increases with increasing particle size in a very similar manner for both the experimental and simulated results. Also, with these results we could verify that the system could measure down to $10 \mathrm{aF}$. 


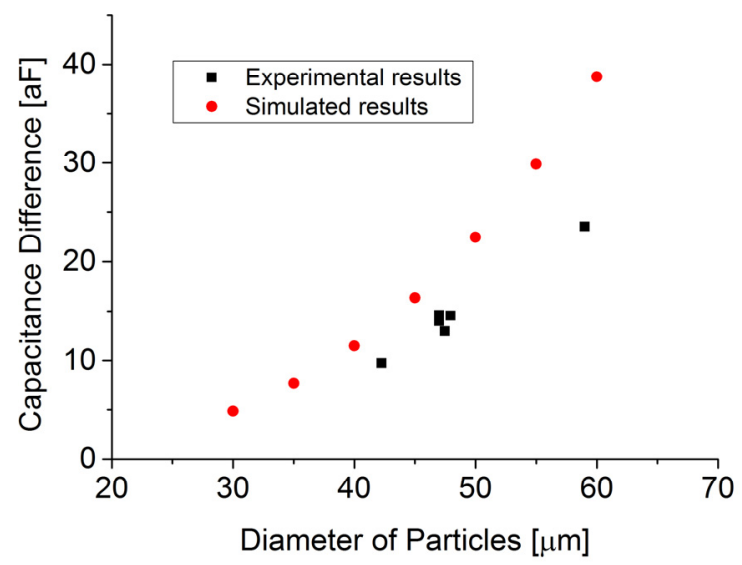

Figure 5.8. Simulated and experimental difference in capacitance of polystyrene particles in anisole.

Thereafter, the dielectric properties of FCC particles were tested. Based on the bode plot previously simulated ${ }^{21}$, see Figure C.3 in Appendix, $50 \mathrm{kHz}$ and $10 \mathrm{MHz}$ were the frequencies chosen to measure the resistance and capacitance of the system, respectively. The experimental opacity obtained when measuring the impedance of LML and HML ECAT FCC particles is presented in Figure 5.9 showing very scattered results and no clear distinction between the slopes of both type of particles. This is probably caused by the heterogeneity of the catalyst itself. Also, when looking at Figure 5.9, differences in capacitance in the same type of ECAT FCC particles are much larger than those averaged between different types of ECAT FCC particles.

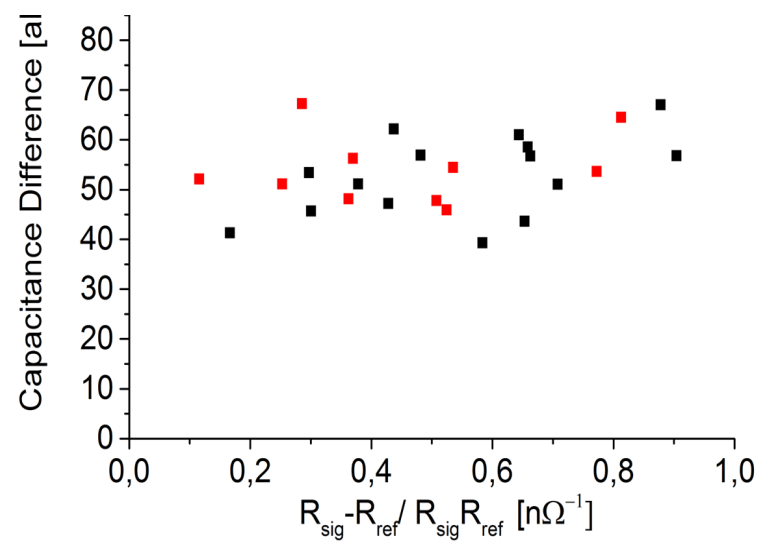

Figure 5.9. Opacity, capacitance versus relative resistance difference of LML and HML FCC particles. 


\subsection{Conclusions}

In conclusion, a microfluidic chip was fabricated able to measure differences in impedance when a particles flows between the electrodes down to $10 \mathrm{aF}$. The impedance of ECAT FCC particles at high and low frequencies was measured showing no differences between particles with different metal loading. Furthermore, when measuring the impedance change of particles flowing between the electrodes, a notable current peak was observed. A simulation was developed demonstrating that the ground electrodes and the position of the particles inside the channel caused these peaks.

\subsection{References}

1. Vogt, E. T. C. \& Weckhuysen, B. M. Fluid catalytic cracking: recent developments on the grand old lady of zeolite catalysis. Chem. Soc. Rev. 44, 7342-7370 (2015).

2. Komvokis, V., Tan, L. X. L., Clough, M., Pan, S. S. \& Yilmaz, B. Zeolites in Fluid Catalytic Cracking (FCC). in Zeolites in Sustainable Chemistry 271297 (Springer, 2016).

3. Dyrkacz, G. R., Ruscic, L., Marshall, C. L. \& Reagan, W. Separation and Characterization of FCC Catalysts Using Density Gradient Separation. Energy E Fuels 71, 849-854 (2000).

4. Sadeghbeigi, R. Fluid Catalytic Cracking Handbook. An expert guide to the practical operation, design and optimization of FCC units. (Elsevier).

5. Chang, A. Y. \& Lu, M. S. C. A CMOS magnetic microbead-based capacitive biosensor array with on-chip electromagnetic manipulation. Biosens. Bioelectron. 45, 6-12 (2013).

6. Mohammad, K., Buchanan, D. A., Braasch, K., Butler, M. \& Thomson, D. J. CMOS single cell dielectrophoresis cytometer. Sensors Actuators, B Chem. 249, 246-255 (2017).

7. Manaresi, N. et al. A CMOS Chip for Individual Cell Manipulation and Detection. IEEE J. Solid-State Circuits 38, 2297-2305 (2003).

8. Chung, J., Chen, Y. \& Kim, S. J. High-density impedance-sensing array on complementary metal-oxide-semiconductor circuitry assisted by negative dielectrophoresis for single-cell-resolution measurement. Sensors Actuators, B Chem. 266, 106-114 (2018).

9. Wei, J., Yue, C., Chen, Z. L., Liu, Z. W. \& Sarro, P. M. A silicon MEMS structure for characterization of femto-farad-level capacitive sensors with lock-in architecture. J. Micromechanics Microengineering 20, (2010).

10. Chen, Y. et al. CMOS high density electrical impedance biosensor array for tumor cell detection. Sensors Actuators, B Chem. 173, 903-907 (2012).

11. De Wagenaar, B. et al. Towards microfluidic sperm refinement: Impedance-based analysis and sorting of sperm cells. Lab Chip 16, 15141522 (2016). 
12. de Wagenaar, B. et al. Spermometer: electrical characterization of single boar sperm motility. Fertil. Steril. 106, 773-780.e6 (2016).

13. Chen, S. C., Le, D. K. \& Nguyen, V. S. Inductive displacement sensors with a notch filter for an active magnetic bearing system. Sensors (Switzerland) 14, 12640-12657 (2014).

14. Konczakowska, A. \& Wilamowski, B. M. Noise in Semiconductor Devices. in Industrial Electronics Handbook, vol. 1 - Fundamentals of Industrial Electronics chapter 11, pp. 11-1-12 (2011).

15. Gawad, S., Cheung, K., Seger, U., Bertsch, A. \& Renaud, P. Dielectric spectroscopy in a micromachined flow cytometer: theoretical and practical considerations. Lab Chip 4, 241-51 (2004).

16. Meirer, F. et al. Life and death of a single catalytic cracking particle. Sci. Adv. 1, e1400199 (2015).

17. Vilkner, T., Janasek, D. \& Manz, A. Micro total analysis systems. Recent developments. Anal. Chem. 76, 3373-3386 (2004).

18. Brookhuis, R. A., Lammerink, T. S. J. \& Wiegerink, R. J. Differential capacitive sensing circuit for a multi-electrode capacitive force sensor. Sensors Actuators, A Phys. 234, 168-179 (2015).

19. Van Der Helm, M. Electrical and microfluidic technologies for organs-on-chips Mimicking blood-brain barrier and gut tissues. (2018).

20. Cheever, E. An Algorithm for Modified Nodal Analysis. Swarthmore University Available at: https://www.swarthmore.edu/NatSci/ echeeve1/Ref/mna/MNA3.html.

21. Sun, T., Bernabini, C. \& Morgan, H. Single-colloidal particle impedance spectroscopy: Complete equivalent circuit analysis of polyelectrolyte microcapsules. Langmuir 26, 3821-3828 (2010). 


\section{Chapter

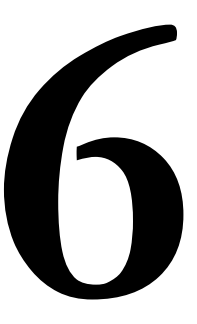

\section{Gradient in Electric Field for Particle Position Detection in Microfluidic Channels}

Tn this chapter, a new method to track particles in microfluidic channels is presented.

Particle position detection in microfluidic systems is crucial to characterize sorting systems or to improve the analysis of cells in impedance flow cytometry studies. By developing an electric field gradient in a two parallel electrodes array the position of the particles can be tracked in one axis by impedance analysis. This method can track the particle's position at lower frequencies and measure the conductivity of the system at higher frequencies. A 3-D simulation was performed showing particle position detection and conductivity analysis. To experimentally validate the technique, a microfluidic chip that develops a gradient in the electric field was fabricated and used to detect the position of polystyrene particles in one axis and measure their conductivity at low and high frequencies, respectively.

This chapter is based on:

Solsona, M.*, Westerbeek, E.Y.*, Bomer, J.C., Olthuis, W., van den Berg, A. Gradient in Electric Field for Particle Position Detection in Microfluidic Channels, Lab on a chip, Lab on a chip, 2019,19, 10541059.

* Both authors contributed equally to this work 


\subsection{Introduction}

Microfluidic single cell analysis has become an important tool in biology. The high throughput and better control that microfluidics enables can provide more precise information about microstructures such as cells. ${ }^{1-4}$ Single cell microfluidic analysis is divided into two areas: cell separation and characterization. Many techniques have been developed to sort cells/particles during the last two decades, from passive methods as pinched and dean flow fractionation (PFF) and (DFF) respectively, ${ }^{5-8}$ to active methods that use magnetic ${ }^{9-11}$ or acoustic fields ${ }^{12,13}$ to push or pull cells/particles or liquids in microfluidic channels. The initial position of those cells before the separation process is of great importance for the final result and there is still very little literature on the study of the separation effectiveness.

Among others, impedance flow cytometry, which is the study of the electrical properties of cells, has become a widely used label-free analysis method. This method consists of applying an alternating voltage to a pair of electrodes and measuring the resulting current and its dependence on frequency. This impedance-based technique provides high throughput ${ }^{14-27}$ which is necessary when dealing with samples of thousands of cells. In cell impedance flow cytometry, the position of the cell between the electrodes is of importance due to inhomogeneities in the electric field. ${ }^{28}$ This could be avoided by flow-focusing the particles in the centre of the electrodes, however, this requires the use of more equipment and larger electrolyte volumes. ${ }^{29,30}$ Also, external forces that use electric, magnetic or acoustic fields can manipulate cells/particles, ${ }^{15}$ however this involves more complex systems. Sometimes, for simplicity, impedance flow cytometry studies avoid particle focusing systems, lowering the quality of the information obtained. A solution to this problem consists of measuring the position of particles in microfluidic systems. By doing so, the measured impedance can be corrected for the cell/particle's position providing more accurate information. ${ }^{31}$ In general, new methods are needed to track the position of cells/particles inside microfluidic channels.

Traditional cell/particle position detection in microfluidic channels is done by optical means, ${ }^{32-34}$ however, sometimes this can be very computer power consuming. ${ }^{35}$ Another method that enables high throughput is microfluidic impedance spectrometry. When used in impedance flow cytometry studies, this technique doesn't require new equipment. Various impedimetric systems able to detect particle's position in one axis of the microchannel have been developed. ${ }^{31,36-40}$ More recently, a combination of these techniques has been used to detect the position of particles in channels in two axis using 10 electrodes. ${ }^{41}$

In this study we developed a new technique that uses microfluidic impedance spectrometry to detect the position of micro-particles in one axis. Previous work in our group demonstrated that a gradual capacitance in a two 
parallel electrodes array could detect the position of objects in one axis. ${ }^{42}$ However, this first approach was tested in a big $(2 \times 2 \times 3 \mathrm{~cm})$ electrochemical cell and no-flow conditions. Our aim is to use the same method to detect the position of microparticles in microchannels in flow conditions. The technique consists of creating a gradual increasing effective area on the electrodes by an increasing area electrodeposited of a conductive material and, hence, gradually increasing the effective capacitance of the electrodes. Figure 6.1a shows a simplified electrical equivalent circuit of the system consisting of different parallel branches with an increasing electrodeposited area. As can be observed, the particle increases the resistance in that region and, and due to the electric field gradient its position can be detected. Figure 6.1b shows a 2-D Comsol simulation where the electrodes on both sides have an increasing linear electrodeposited area in the same direction, from bottom to top. The benefit of this technique is the double information that can be obtained at two different frequencies. At low frequencies, when most of the current flows through the areas with a bigger electrodeposited area, hence a lower impedance, a gradient in electric field is formed and the position of the microparticles can be detected. At this frequencies both the impedance of the medium and the capacitors are of the same range, thus providing the electric field gradient. On the other hand, at higher frequencies, the impedance of all the capacitors is much lower than the impedance of the medium. Here, the current density is homogeneous through the whole electrochemical cell, therefore the conductivity of the system and thus, those of the particles, can be measured.

In this work, a finite element model (FEM) was developed to demonstrate the frequency range at which the position and dielectric properties of the particles can be detected and measured. Also, to validate the FEM, a microfluidic chip with a gradient in electrodeposited area perpendicular to the flow direction was fabricated and experimental results are shown. 
(a)

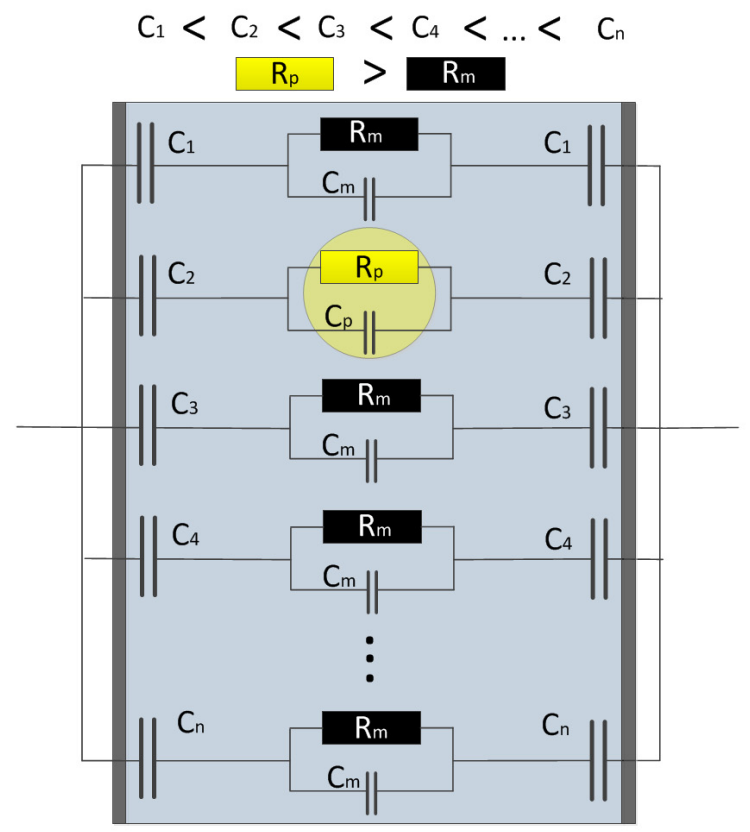

(b)

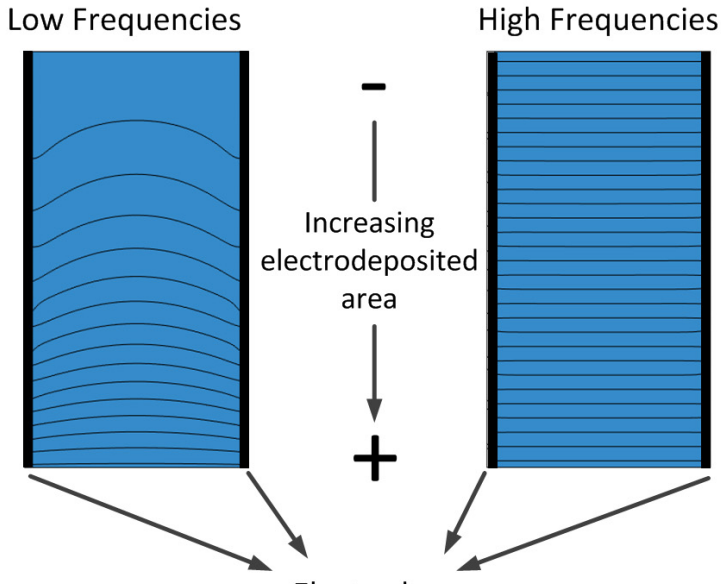

Electrodes

Figure 6.1. (a) Simplified electrical equivalent circuit of a parallel electrodes array with a gradient in electrodeposited area at both electrodes. (b) Current density simulation of a two platinum electrodes with a linear increasing electrodeposited area at lower and higher frequencies. 


\subsection{Materials and methods}

A 3-D FEM simulation was performed using the electrochemistry package of Comsol Multiphysics. A microfluidic glass-SU-8-glass chip was developed using standard photo-lithographic techniques, Figure D.1a in Appendix. In short, the parallel electrodes were fabricated by patterning photoresist, wet etching $140 \mathrm{~nm}$ and sputtering $10 \mathrm{~nm}$ of Tantalum, as an adhesion layer, and $130 \mathrm{~nm}$ of Gold on both wafers, and lift-off the resist. Thereafter, another positive photoresist pattern was applied to uncover specific areas on the electrodes, those to be electrodeposited, which increased in size through the $x$-direction to have an increasing electrodeposited area Figure 6.2. These areas were thereafter cleaned performing cyclic voltammetry from -500 to $800 \mathrm{mV}$ and a scan rate of $10 \mathrm{mV} / \mathrm{s}$, using a $\mathrm{Ag} / \mathrm{AgCl}$ reference electrode and $0.1 \mathrm{M} \mathrm{H}_{2} \mathrm{SO}_{4}$ solution, see Figure D.2 in Appendix. Subsequently, the exposed areas were electrodeposited with a $2.4 \mathrm{mM}$ Chloroplatinic Acid $\left(\mathrm{H}_{2} \mathrm{PtCl}_{4}\right)$ and $0.8 \mathrm{mM}$ Lead Acetate $\left(\mathrm{Pb}\left(\mathrm{C}_{2} \mathrm{H}_{3} \mathrm{O}_{2}\right)_{2}\right)$ solution.

The electrodeposition was performed in an ultrasonic bath ${ }^{43}$ using a platinum sputtered $10 \mathrm{~cm}$ in diameter wafer as a counter electrode, a $\mathrm{Ag} / \mathrm{AgCl}$ electrode as a reference and applying 50 cycles of pulses consisting of 6 seconds at $500 \mathrm{mV}$ followed by 0.5 seconds at $-100 \mathrm{mV}$. Figure 6.2a presents a gold electrode after electrodeposition showing an increasing electrodeposited area in $\mathrm{x}$-direction. Figure 6.2b presents Scanning Electron Microscope (SEM) images of the electrodeposited electrode showing the typical cauliflower shape of platinum black.

To determine the thickness of the electrodeposited layer, a cross section SEM image was made, Figure 6.2c, showing a thickness of $1 \mu \mathrm{m}$ which is less than $0.5 \%$ of the total height(y-direction) of the channel. In order to bond the 2 wafers, 2 layers of SU- 8 were used. First a $180 \mu \mathrm{m}$ followed by a $35 \mu \mathrm{m}$ SU-8 thick layers were spun on top of each other in order to decrease the roughness of the SU-8 layer and, therefore, accomplish a better and more homogeneous bonding. Both wafers were manually aligned and consequently bonded by applying a pressure of $4000 \mathrm{~N}$ at $120^{\circ} \mathrm{C}$. 
(a)

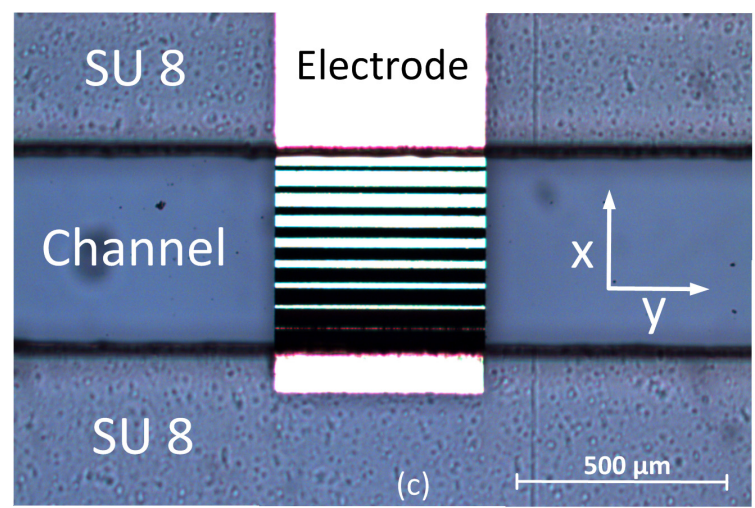

(b)

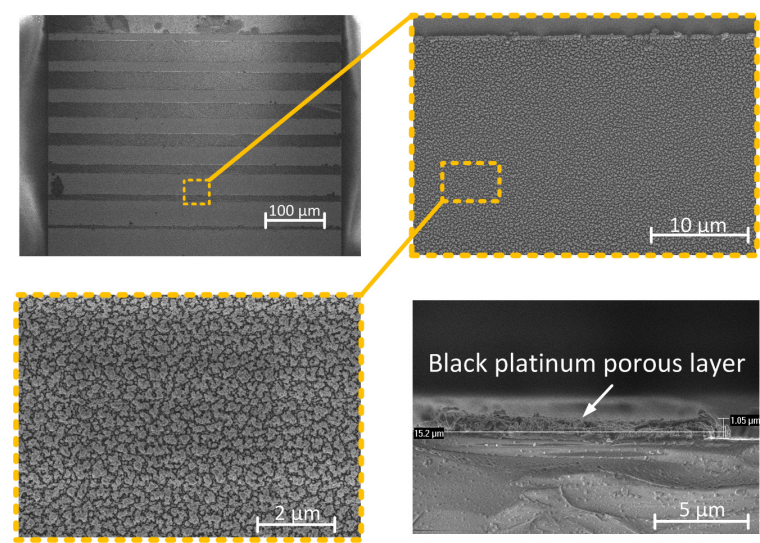

Figure 6.2. (a) Optical image of a microfluidic channel with a gold electrode that has an increasing area from bottom to top electrodeposited with platinum black. (b) Scaning electron microscope (SEM) images of the electrodeposited areas and (c) the thickness of the platinum black layer.

A Micronit sideconnect chip-holder was used to perform the experiments, Figure D.1b in Appendix. The impedance of the system was measured using a Zurich Instruments HF2IS potentiostat with a HF2TA amplifier. The particles were observed and recorded using a high speed camera Grasshopper 3 and flow-focused in the chip using 2 Harvard microfluidic pumps. The electrolyte conductivity was measured with a Mettler Toledo conductivity meter. Polystyrene beads $83 \mu \mathrm{m}$ in diameter were purchased from MicrospheresNanospheres and their size was measured with Leika DM6000M microscope LAS V4.12 software. 
(a)

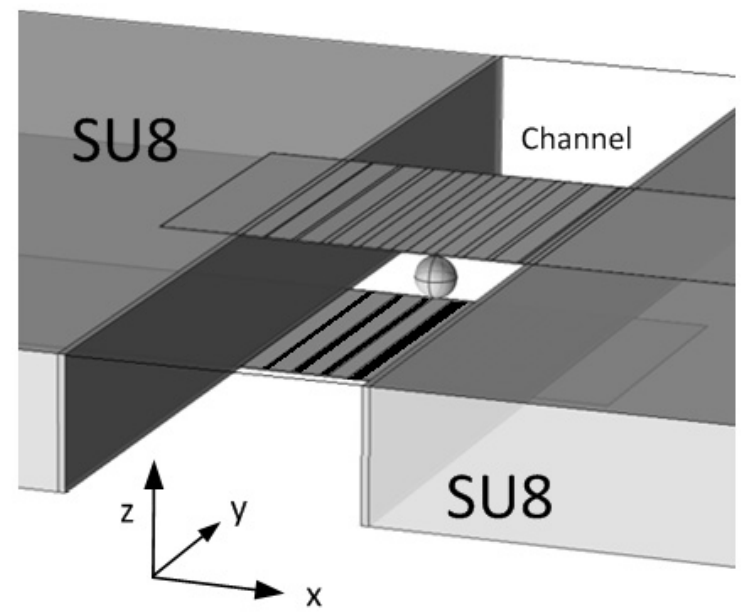

(b)

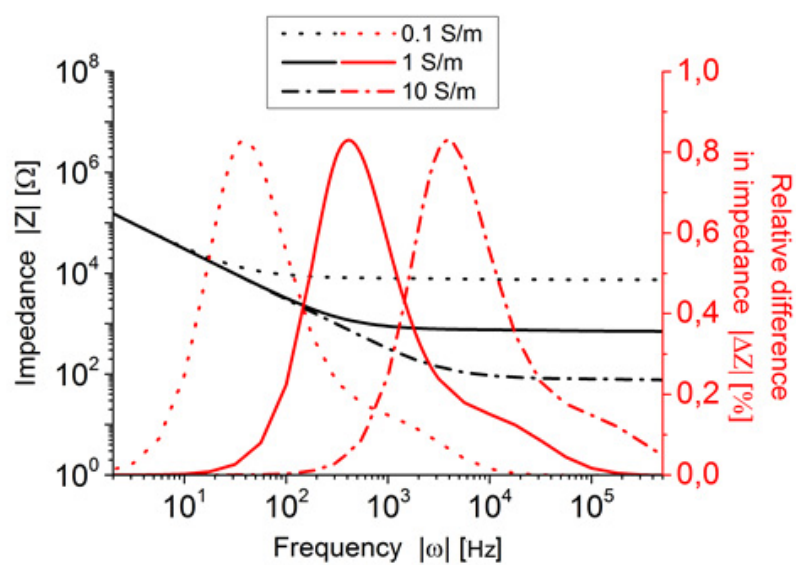

Figure 6.3. (a) Schematic of the FEM design used to simulate the system. On both sides 2 layers of SU-8 are simulated and 2 layers of glass at top and bottom of the channel are simulated but not shown. (b) Three simulated bode plots and difference in relative impedance with conductivities of 0.1, 1 and $10 \mathrm{~S} / \mathrm{m}$ of two polystyrene particles $83 \mu \mathrm{m}$ in diameter at the top and bottom positions in $x$-direction in the microfluidic channel.

\subsection{Results and discussion}

\subsubsection{FEM Simulation}

Figure 6.3a shows the design of the electrochemical cell where the gradient (x-direction) is perpendicular to the channel flow (y-direction). Both side walls are made of SU-8. Top and bottom glass layers are not shown but taken into account in the simulation. To simulate the system, 4 fitting parameters were experimentally measured: the double layer capacitance, the electrolyte conductivity, the microfluidic channel dimensions (see Figure D.3 in Appendix) and the polystyrene particles size. The double layer capacitance of the system was obtained by measuring the impedance at very low frequencies 
$(10 \mathrm{~Hz})$, knowing the high and low capacitance areas and that, at this frequency, the impedance of the system is governed by the double layer capacitance of the electrodes. This resulted in $770 \mu \mathrm{F} / \mathrm{cm}^{2}$ for the electrodeposited areas and 36 $\mu \mathrm{F} / \mathrm{cm}^{2}$ for the plane electrode areas.

The conductivity of the electrolyte was measured and set at $1.06 \mathrm{~S} / \mathrm{m}$, the microfluidic channel dimensions were $219 \mu \mathrm{m}$ height and $477 \mu \mathrm{m}$ wide, see Figure D.3 in Appendix, and the average particle's diameter was $83 \mu \mathrm{m}$, see Figure D.4 in Appendix. Figure 6.3b shows three simulated bode plots of the system with three different electrolyte conductivities, $0.1,1$ and $10 \mathrm{~S} / \mathrm{m}$ where the typical impedance can be seen, dominated by the double layer capacitance at lower frequencies $(<10 \mathrm{~Hz})$ and by the electrolyte resistivity at higher frequencies $(>10000 \mathrm{~Hz})$.

(a)

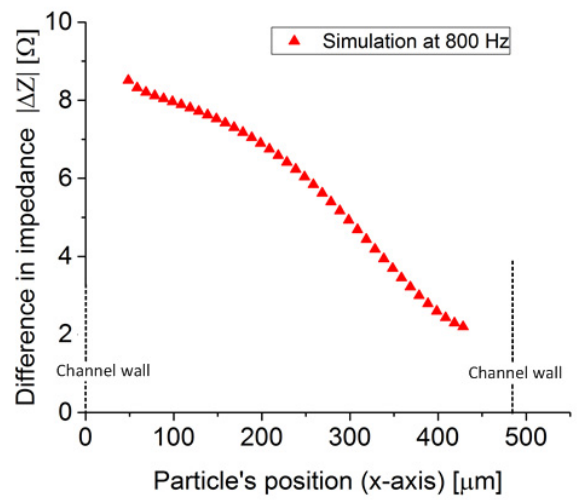

(b)

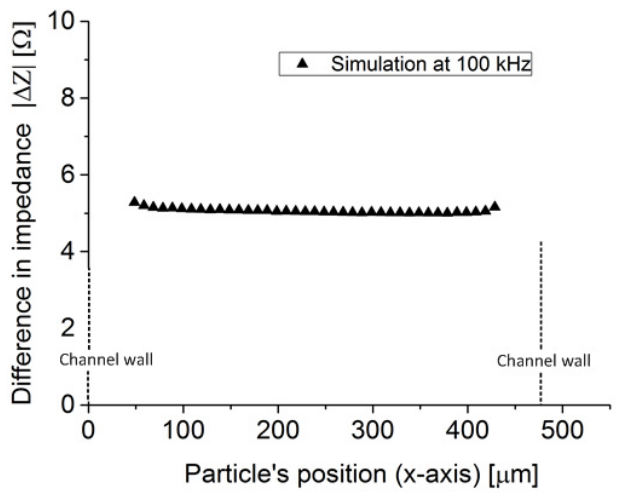

Figure 6.4. Simulated impedance difference of $83 \mu \mathrm{m}$ in diameter polystyrene particles at both (a) $800 \mathrm{~Hz}$ and (b) $100 \mathrm{kHz}$ throughout the whole electrochemical cell (x-direction).

In order to know at which frequency range the particle position has the largest difference in relative impedance due to their position three bode plots with a particle at two extreme $x$-positions in the channel, $x=50 \mu \mathrm{m}$ and $x=440$ $\mu \mathrm{m}$, were simulated. Figure $6.3 \mathrm{~b}$ presents the difference in impedance over the total impedance of the system for both positions showing peaks close to the their respective RC (Resistance-Capacitance) time constant frequency.

To know the impedance dependence on the particle's position, the simulated particle was placed at 40 different $x$-direction positions and the impedance was simulated at $800 \mathrm{~Hz}$ and $100 \mathrm{kHz}$, low and high frequencies respectively. The $z$ and $y$-positions, see Figure 6.4a, were maintained constant at the middle of the channel and electrodes. Figure 6.4a shows that at $800 \mathrm{~Hz}$, the impedance of the system is dependent on the particles' position in $\mathrm{x}$ direction. On other hand, as can be seen in Figure $6.4 \mathrm{~b}$, at $100 \mathrm{kHz}$ the total impedance of the system becomes independent of the position of the particle, as expected from Figure 6.4b. It is worth mentioning that $100 \mathrm{kHz}$ was arbitrary 
chosen as high frequency but any other frequency higher and far away from the position detection frequency could be used.

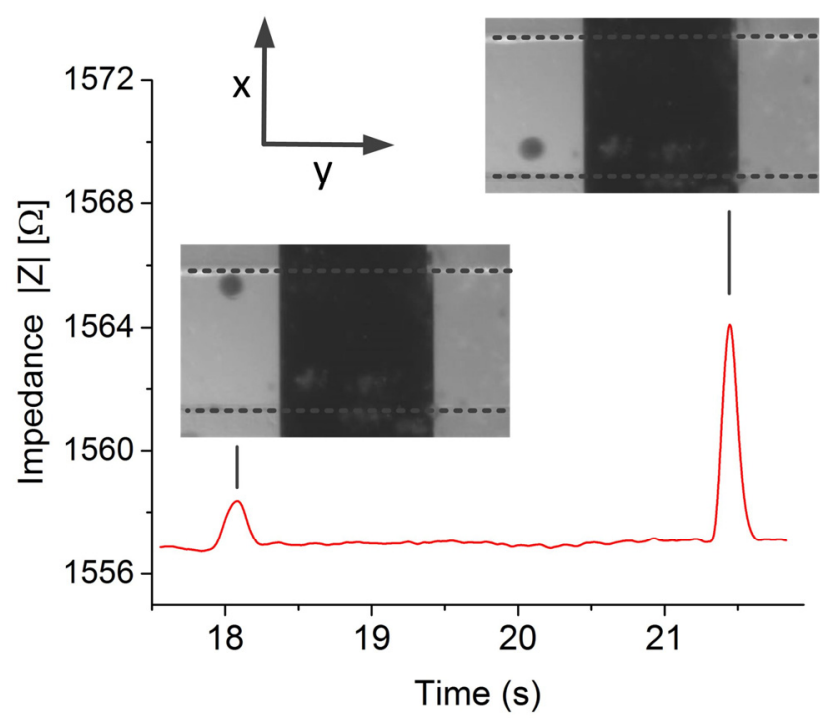

Figure 6.5. Typical experimental data. Images from a video and impedance recorded obtained when measuring the position of particles at low frequencies, $800 \mathrm{~Hz}$.

\subsubsection{Experimental validation}

Figure 6.5 shows the typical data obtained at $800 \mathrm{~Hz}$ of 2 polystyrene particles passing at 2 different positions, top and bottom, in the microfluidic chip using a $1.06 \mathrm{~S} / \mathrm{m}$ conductive electrolyte. The peak heights and width of the signal are related to the position and velocity of the particle respectively. Impedance sample rate was high and particle throughput was low enough to ensure the maximum impedance.

Figure 6.6 presents the measured impedance difference of polystyrene particles at various positions optically determined in x-direction in the microfluidic channel at both frequencies. Also, for comparison purposes, the simulation previously shown is presented. At both frequencies the experimental and simulated data are similar, experimentally demonstrating that the particle's position can be tracked at low frequencies while at higher frequencies the dielectric properties of the system can still be measured. However, when comparing both simulated and experimental data at higher frequencies, $100 \mathrm{kHz}$, an approximately $2 \Omega$ difference in absolute impedance is observed which is the $0.2 \%$ of the total impedance measured. Also, the experimental data shows a $2 \Omega$ spread in impedance difference which could be caused by the broad size distribution of the polystyrene particles resulting in a coefficient of variation of 0.3 . To prove this, 5 simulations with 5 different 
particle sizes, taken from Figure D.4 in Appendix, at high frequencies were performed showing impedance differences up to $\approx 3 \Omega$, Table D.1. Moreover, particles flowing in different positions in the $\mathrm{z}$-direction, which were not tracked in this study, can account for up to $\approx 0.2 \Omega$, Figure D.5 in Appendix. The error of the experimental data was calculated by measuring the average distance to a linear trend line of the experimental data and it resulted in 56.86 $\mu \mathrm{m}$, see Figure D.6 in Appendix. This resulted in 8 different positions that can be tracked without data overlapping which is comparable to the work of Wang et al. ${ }^{36}$, where the authors were able to distinguish between 5 positions in a 188 $\mu \mathrm{m}$ wide channel using 6 and $11 \mu \mathrm{m}$ in diameter particles. The method presented in this work can determine both the position and the conductivity of the system, however it also requires an extra fabrication step compared to the other works.31,36,37 Other comparable studies determined the position of the particle on the z-direction (height of the channel), where the analytical derivation fitted the experimental data throughout all the z-axis between the electrodes.

In order to use the gradient in electric field for cell position detection, the system should be scaled down. In addition, the detection accuracy could be enhanced by measuring the conductivity, which would allow to correct the position detection due to differences in height (z-direction) and size of the cell. It is worth mentioning that the high frequency to measure the conductivity of the system for the correction of the position should be low enough to avoid current flow through the cell, which would disrupt the correction itself.

(a)

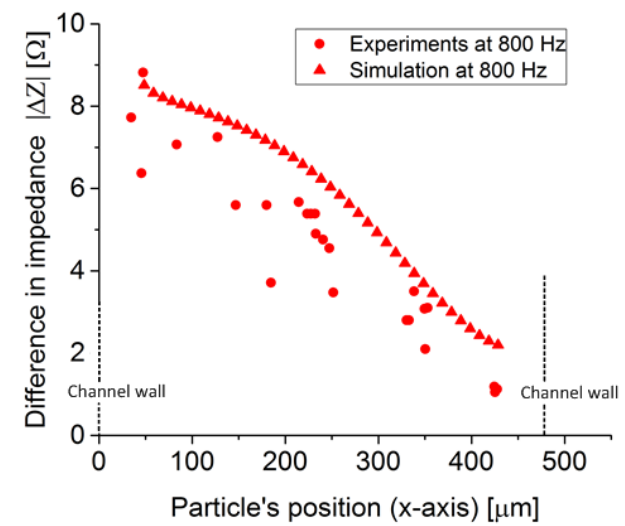

(b)

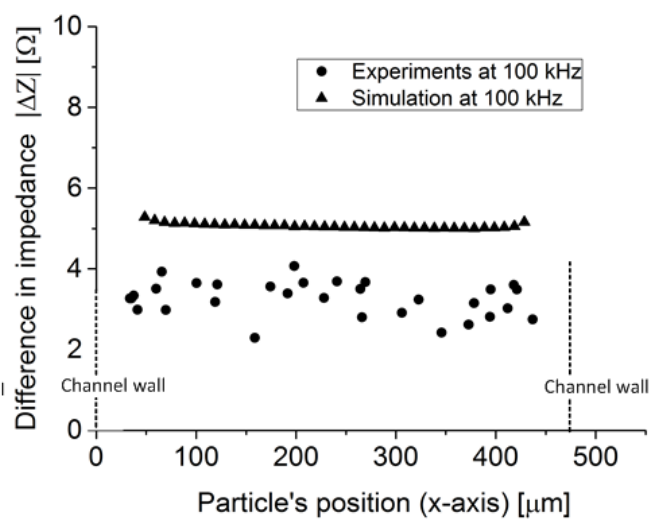

Figure 6.6. Experimental results and simulation of the impedance difference obtained from $83 \mu \mathrm{m}$ polystyrene particles at (a) $800 \mathrm{~Hz}$ and (b) $100 \mathrm{kHz}$. 


\subsection{Conclusions}

In conclusion, we report a new method to track particles in microfluidic devices by using a gradient in electric field at low frequencies $\approx 800 \mathrm{~Hz}$ and measure their conductivity at higher frequencies $\approx 100 \mathrm{kHz}$. $80 \mu \mathrm{m}$ polystyrene particles were optically tracked and correlated to their impedance difference. The experimental results and the FEM at low and high frequencies are similar however the experimental data shows a spread of $2 \Omega$ resulting in a coefficient of variation of $\approx 0.3$. We believe that this technique could be extended to develop a 3-D tracker to detect the position of cells/particles and measure their dielectric properties at high throughput.

\subsection{References}

1. Klein, A. M. et al. Droplet barcoding for single-cell transcriptomics applied to embryonic stem cells. Cell 161, 1187-1201 (2015).

2. Sanchez-Freire, V., Ebert, A. D., Kalisky, T., Quake, S. R. \& Wu, J. C. Microfluidic single-cell real-time PCR for comparative analysis of gene expression patterns. Nat. Protoc. 7, 829-838 (2012).

3. Marcus, J. S., Anderson, W. F. \& Quake, S. R. Microfluidic single-cell mRNA isolation and analysis. Anal. Chem. 78, 3084-3089 (2006).

4. de Wagenaar, B. et al. Spermometer: electrical characterization of single boar sperm motility. Fertil. Steril. 106, 773-780.e6 (2016).

5. Nivedita, N., Ligrani, P. \& Papautsky, I. Dean Flow Dynamics in LowAspect Ratio Spiral Microchannels. Sci. Rep. 7, 1-10 (2017).

6. Morijiri, T., Sunahiro, S., Senaha, M., Yamada, M. \& Seki, M. Sedimentation pinched-flow fractionation for size- and density-based particle sorting in microchannels. Microfluid. Nanofluidics 11, 105-110 (2011).

7. Amini, H., Lee, W. \& Di Carlo, D. Inertial microfluidic physics. Lab Chip 14, 2739-2761 (2014).

8. Bhagat, A. A. S., Kuntaegowdanahalli, S. S. \& Papautsky, I. Continuous particle separation in spiral microchannels using dean flows and differential migration. Lab Chip 8, 1906-1914 (2008).

9. Pamme, N. \& Manz, A. On-chip free-flow magnetophoresis: Continuous flow separation of magnetic particles and agglomerates. Anal. Chem. 76, 7250-7256 (2004).

10. Furlani, E. P. Analysis of particle transport in a magnetophoretic microsystem. J. Appl. Phys. 99, 1-35 (2006).

11. Solsona, M. et al. Magnetophoretic Sorting of Single Catalyst Particles. Angew. Chemie - Int. Ed. 57, 10589-10594 (2018).

12. Li, P. et al. Acoustic separation of circulating tumor cells. Proc. Natl. Acad. Sci. 112, 4970-4975 (2015).

13. Shi, J., Huang, H., Stratton, Z., Huang, Y. \& Huang, T. J. Continuous 
particle separation in a microfluidic channel via standing surface acoustic waves (SSAW). Lab Chip 9, 3354-3359 (2009).

14. Gawad, S., Cheung, K., Seger, U., Bertsch, A. \& Renaud, P. Dielectric spectroscopy in a micromachined flow cytometer: theoretical and practical considerations. Lab Chip 4, 241-51 (2004).

15. Mernier, G., Duqi, E. \& Renaud, P. Characterization of a novel impedance cytometer design and its integration with lateral focusing by dielectrophoresis. Lab Chip 12, 4344-4349 (2012).

16. McGrath, J. S. et al. Analysis of Parasitic Protozoa at the Single-cell Level using Microfluidic Impedance Cytometry. Sci. Rep. 7, 1-11 (2017).

17. Xavier, M., De Andrés, M. C., Spencer, D., Oreffo, R. O. C. \& Morgan, H. Size and dielectric properties of skeletal stem cells change critically after enrichment and expansion from human bone marrow: consequences for microfluidic cell sorting. Size and dielectric properties of skeletal stem cells change critically after enrich. J. R. Socciety Interface 14, (2017).

18. Zhao, Y. et al. Development of micro fluidic impedance cytometry enabling the quantification of specific membrane capacitance and cytoplasm conductivity from 100,000 single cells. 111, 138-143 (2018).

19. Bürgel, S. C., Escobedo, C., Haandbæk, N. \& Hierlemann, A. Chemical On-chip electroporation and impedance spectroscopy of single-cells. Sensors Actuators B Chem. 210, 82-90 (2015).

20. De Wagenaar, B. et al. Towards microfluidic sperm refinement: Impedance-based analysis and sorting of sperm cells. Lab Chip 16, 15141522 (2016).

21. Segerink, L. I., Sprenkels, A. J., Ter Braak, P. M., Vermes, I. \& Van Den Berg, A. On-chip determination of spermatozoa concentration using electrical impedance measurements. Lab Chip 10, 1018-1024 (2010).

22. Chien, J. C. et al. A high-throughput flow cytometry-on-a-CMOS platform for single-cell dielectric spectroscopy at microwave frequencies. Lab Chip 18, 2065-2076 (2018).

23. Chung, J., Chen, Y. \& Kim, S. J. High-density impedance-sensing array on complementary metal-oxide-semiconductor circuitry assisted by negative dielectrophoresis for single-cell-resolution measurement. Sensors Actuators, B Chem. 266, 106-114 (2018).

24. Manaresi, N. et al. A CMOS Chip for Individual Cell Manipulation and Detection. IEEE J. Solid-State Circuits 38, 2297-2305 (2003).

25. Mohammad, K., Buchanan, D. A., Braasch, K., Butler, M. \& Thomson, D. J. CMOS single cell dielectrophoresis cytometer. Sensors Actuators, B Chem. 249, 246-255 (2017).

26. Chen, Y. et al. CMOS high density electrical impedance biosensor array for tumor cell detection. Sensors Actuators, B Chem. 173, 903-907 (2012).

27. Chang, A. Y. \& Lu, M. S. C. A CMOS magnetic microbead-based capacitive biosensor array with on-chip electromagnetic manipulation. 
Biosens. Bioelectron. 45, 6-12 (2013).

28. Spencer, D. \& Morgan, H. Positional dependence of particles in microfludic impedance cytometry. Lab Chip 11, 1234 (2011).

29. Spielman, L. \& Goren, S. L. Improving resolution in coulter counting by hydrodynamic focusing. J. Colloid Interface Sci. 26, 175-182 (1968).

30. Zhao, J. \& You, Z. Microfluidic hydrodynamic focusing for highthroughput applications. J. Micromechanics Microengineering 25, (2015).

31. Caselli, F., De Ninno, A., Reale, R., Businaro, L. \& Bisegna, P. A novel wiring scheme for standard chips enabling high-accuracy impedance cytometry. Sensors Actuators, B Chem. 256, 580-589 (2018).

32. Tapson, J. \& Greene, J. R. Improved capacitance measurement by means of resonance locking. Meas. Sci. Technol. 5, 20-26 (1994).

33. Fang, Y., Yu, N., Jiang, Y. \& Dang, C. High-precision lens-less flow cytometer on a chip. Micromachines 9, 1-13 (2018).

34. Vercruysse, D. et al. Three-part differential of unlabeled leukocytes with a compact lens-free imaging flow cytometer. Lab Chip 15, 1123-1132 (2015).

35. Winer, M. H., Ahmadi, A. \& Cheung, K. C. Application of a threedimensional (3D) particle tracking method to microfluidic particle focusing. Lab Chip 14, 1443-1451 (2014).

36. Wang, H., Sobahi, N. \& Han, A. Impedance spectroscopy-based cell/particle position detection in microfluidic systems. Lab Chip 17, 12641269 (2017).

37. Brazey, B. et al. Impedance-based real-time position sensor for lab-on-achip devices. Lab Chip 18, 818-831 (2018).

38. De Ninno, A. et al. Coplanar electrode microfluidic chip enabling accurate sheathless impedance cytometry. Lab Chip 17, 1158-1166 (2017).

39. Errico, V. et al. Mitigating positional dependence in coplanar electrode Coulter-type microfluidic devices. Sensors Actuators, B Chem. 247, 580-586 (2017).

40. Spencer, D., Caselli, F., Bisegna, P. \& Morgan, H. High accuracy particle analysis using sheathless microfluidic impedance cytometry. Lab Chip 16, 2467-2473 (2016).

41. Reale, R., De Ninno, A., Businaro, L., Bisegna, P. \& Caselli, F. Electrical measurement of cross-sectional position of particles flowing through a microchannel. Microfluid. Nanofluidics 22, 1-13 (2018).

42. Solsona, M., Olthuis, W. \& Van Den Berg, A. Gradient Capacitance for Solid Particle Position Detection in Electrolyte. Procedia Eng. 168, 14461449 (2016).

43. Desai, S. A., Rolston, J. D., Guo, L. \& Potter, S. M. Improving impedance of implantable microwire multi-electrode arrays by ultrasonic electroplating of durable platinum black. Front. Neuroeng. 3, 1-11 (2010). 



\section{Chapter

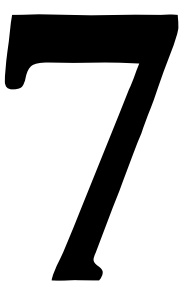

\section{Ion Concentration Polarization for Microparticle Mesoporosity Differentiation}

M

icroparticle porosity is normally determined in bulk manner providing an ensemble average that hinders establishing the individual role of each microparticle. On the other hand, single particle characterization implies expensive technology. We propose to use ion concentration polarization to measure differences in meso-porosity at single particle level. Ion concentration polarization occurs at the interface between an electrolyte and a porous particle when an electric field is applied. The extent of ion concentration polarization depends, amongst others, on the nanopore size and density. By using a fluorescence marker we could measure differences in concentration polarization between particles with $3 \mathrm{~nm}$ and $13 \mathrm{~nm}$ average mesopore diameters. A qualitative model was developed in order to understand and interpret the phenomena. We believe that this inexpensive method could be used to measure differences in mesoporous particle materials such as catalysts

This chapter is based on:

Solsona, M.*, Papadimitriou, V.A.*, Olthuis, W., van den Berg, A., Eijkel, J.C.T., Ion Concentration Polarization for Microparticle Mesoporosity Differentiation. Submitted

* Both authors contributed equally to this work 


\subsection{Introduction}

Porous materials are key components in many fields that rely on their void volume, network distribution, and pore size and shape. ${ }^{1-3}$ They are particularly interesting in applications such as: catalysis, supercapacitors, fuel cells and batteries, due to their unique interaction with atoms, ions and molecules at the nanometer and sub-nanometer scale. In chemistry, in particular heterogeneous catalysis, the porosity of solid materials is normally used to enhance the available area in contact with reagents and products, thus increasing the activity per unit volume. ${ }^{4}$ In practice a porous catalyst is used or a catalyst support with immobilized nanoparticles. However, small differences in the porosity of these materials can cause large variations in mass transport of reagents through the pores which can result in a reduction or even loss of catalyst activity. ${ }^{5-7}$ Normally, these catalysts or catalyst supports are fabricated in batch reactors in a non-controlled but reproducible manner, resulting in large inter- and intra-particle heterogeneities.

Nanopores are generally subdivided into three groups depending on the pore dimensions: micro $(<2 \mathrm{~nm})$, meso $(2-50 \mathrm{~nm})$ and macropores $(>50 \mathrm{~nm})$. There are many techniques available to measure the pore size distribution of nanoporous materials, where the most popular ones are based on gas adsorption, fluid intrusion, microscopy and spectroscopy. 5,8-12 The BrunauerEmmett-Teller (BET) method uses the adsorption of chemically inert gases on the porous material walls to determine the available surface area in the volume measured. ${ }^{13}$ The pore size distribution can also be derived from the pressure needed to drive flow of fluids such as $\mathrm{Hg}$ into the porous media. ${ }^{14}$ Both methods are widely used but the volumes of porous material needed are large, providing an ensemble averaged porosity instead of the porosity of single microparticles. For the latter, amongst others, Scanning Electron Microscopy (SEM) and X-Ray diffraction are used to determine meso- and macro-porosity at single microparticle level. These methods, however, imply the use of expensive technology and can study only very small quantities. 7,15,16 Therefore, new ways to measure the porosity of particles at the single-particle level are needed in order to understand and increase the efficiency of porous systems. In this study, Ion Concentration Polarization (ICP) is used to measure the mesoporosity at single particle level . ICP occurs at the interfaces of an ion-permselective material and free solution on application of an electrical field. ${ }^{17-19}$ It results in the formation of ion accumulation and depletion zones at the opposite sides of the material due to the ion flux imbalance between free solution and inside the particle, see Figure 7.1a. Porous materials become ion-permselective when their pore dimensions are of the order of the electrical double layer thickness at the pore walls (typically nanometer scale).

Here, we propose to use ICP in a microfluidic system to analyse the mesoporosity of two types of porous polystyrene particles with different 
mesoporosities. We first present an approximative theory describing ICP at single particle level. Then, we show measurements and analysis of the ICP experimental fluorescent response of both types of particles at different electrolyte concentrations using a fluorescent dye marker with constant concentration.

(a)
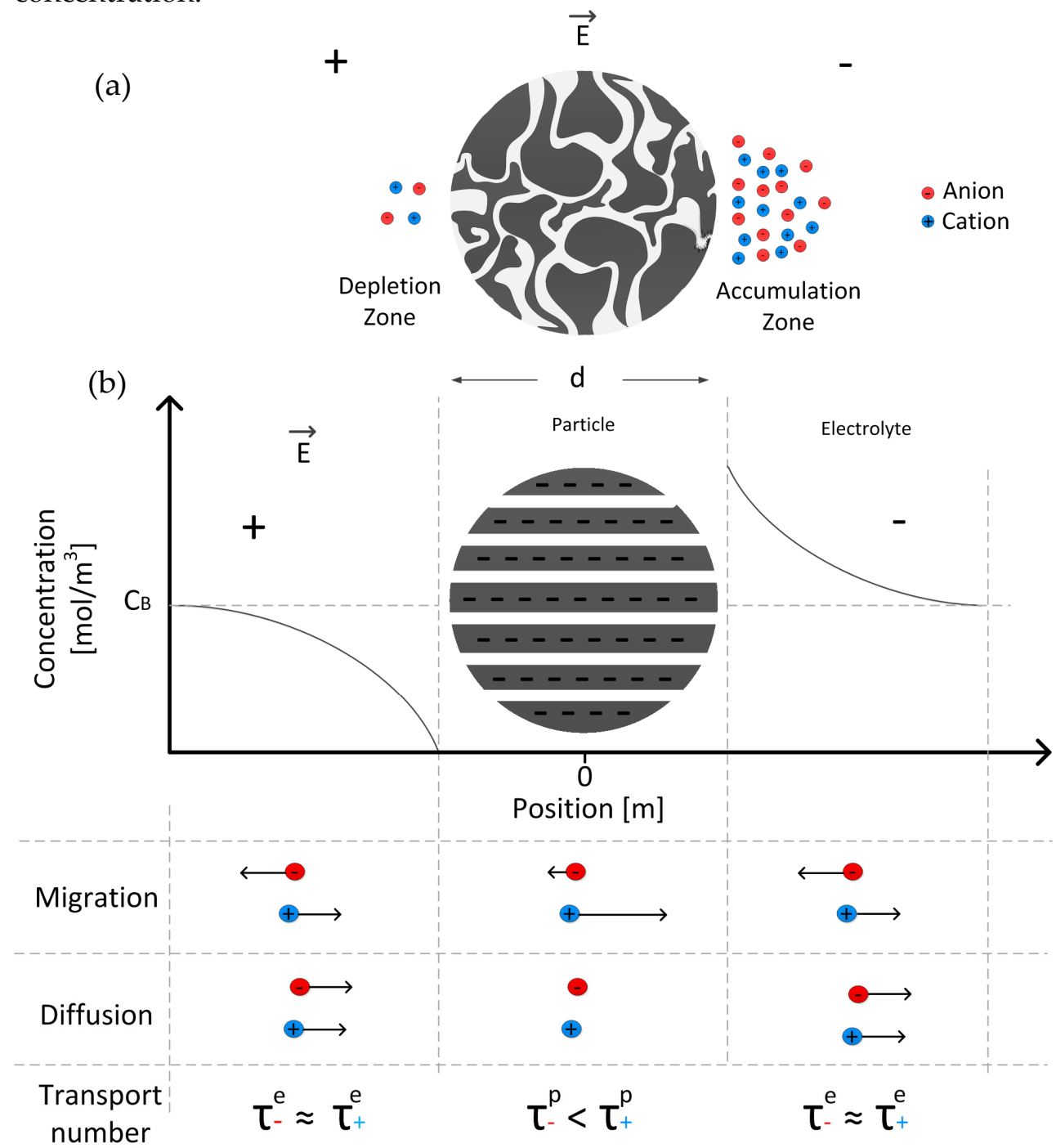

Figure 7.1. (a) Schematic of ICP at a cation-selective porous particle. The depletion and accumulation zones are indicated. (b) Schematic of the porous particle under an electric field. ICP occurs as a result of the ion flux imbalance at the particle/solution interfaces as shown by the flux vectors and the electrical transport numbers $\tau$ in the particle and solution. At the anodic side, a depletion zone is formed close to the interface of the particle while an enrichment zone is formed at the cathodic side. For simplicity a solution is assumed with a 1:1 electrolyte. 


\subsection{Theory}

Figure $7.1 \mathrm{~b}$ shows the mechanism of ICP, occurring when a uniform electric field is applied across a porous ion-permselective particle. ICP occurs when a difference exists in the fraction of the current that is carried by the different ionic species in particle and bulk solution, and involves gradients in the migration and diffusion fluxes. The ion permselectivity of the particles is caused by the difference in ionic concentration composition in the mesopores and the bulk. Assuming negative surface charge on the pore walls, more cations are present in the solution in the pores due to electroneutrality, and therefore more current will be carried by the cations in the pores than by the anions when compared to the bulk solution. A depletion zone, where the concentrations of all ionic species will be lower than in the bulk, will then form at the anodic side of the particle (left side on Figure 7.1b). An accumulation zone, where the concentration of all ionic species will be higher than in the bulk, will form on the cathodic side (right side on Figure 7.1b). In the depletion and accumulation zones additional diffusion fluxes maintain a steady-state constant flux of cations and anions. In order to quantify the extent of ICP we use a fluorescent reporter ion and measure the difference of its fluorescent intensity in the depletion and accumulation zones during $4 \mathrm{sec}$ over several alternating potential cycles assuming a linear relation between fluorescent intensity and concentration of the reporter. $\mathrm{NaCl}$ is used as bulk electrolyte salt (diffusion coefficients of $1.374 \cdot 10^{-5}\left[\mathrm{~cm}^{2} / \mathrm{s}\right]$ and $1.957 \cdot 10^{-5}\left[\mathrm{~cm}^{2} / \mathrm{s}\right]^{20}$ for $\mathrm{Na}$ and $\mathrm{Cl}$ respectively and electrophoretic mobilities calculated with Einstein's relation). We will first theoretically derive the concentration profile of our fluorescent reporter bodipy disulfonate $\left(\mathrm{BDP}^{2-}\right)$ in the solution as a function of time and distance from the particle surface $\left(C_{B D P}(x, t)\right)$, in an approach similar to Bergveld et al. ${ }^{21}$

As our theoretical model is designed only to estimate a qualitative behaviour of our system, a series of strong assumptions are made: i) The model is one-dimensional, ii) the convective flux (electroosmotic flow) can be neglected, iii) the particle pores are assumed to be perfectly cylindrical running the whole length of the particle, iv) the particle is assumed to be rectangular with a length equal to the diameter of the real particle, $v$ ) the particle is assumed to be a square part of a continuous membrane, with uniform non-crossing cylindrical mesopores passing through it, vi) the concentration distribution at the enrichment and depletion zone does not affect the local electric field, vii) the diffusive flux inside the particle is neglected.

\subsubsection{Nernst-Planck}

To calculate the concentrations of $\mathrm{BDP}^{2-}\left(C_{B D P}(x, t)\right)$, we will use the onedimensional Nernst-Planck flux equations. The current density $\left(j\left[A / m^{2}\right]\right)$, the local conductivity $(\kappa[S / m])$ and the local electric field $(E[\mathrm{~V} / \mathrm{m}])$ are related by 


$$
\kappa(x, t) \boldsymbol{E}(\boldsymbol{x}, \boldsymbol{t})=\boldsymbol{j} .
$$

We will first consider the transport of $\mathrm{BDP}^{2-}$ in the electrolyte bordering the particle. Ignoring the convective flux contribution to the Nernst-Planck equation (hereby included electroosmotic flow), we can write the flux $J^{e}$ of $\mathrm{BDP}^{2-}$ in the electrolyte as composed of a migration and a diffusion term,

$$
\boldsymbol{J}_{B D P}^{e}(x, t)=-\frac{\tau_{B D P}^{e} \boldsymbol{j}}{F}-D \frac{\partial C_{B D P}^{e}(x, t)}{\partial x}
$$

where $\tau_{B D P}^{e}$ is the dimensionless transport number of $\mathrm{BDP}^{2-}$ in the electrolyte, $F$ the faraday constant and $D$ the diffusion coefficient of $\mathrm{BDP}^{2-}$. By applying the continuity equation

$$
\frac{\partial C_{B D P}^{e}(x, t)}{d t}=-\frac{\boldsymbol{J}_{B D P}^{e}(x, t)}{d x}
$$

and realizing that the migration term in the electrolyte is constant in $x$, we arrive at

$$
\frac{\partial C_{B D P}^{e}(x, t)}{d t}=-D \frac{\partial^{2} C_{B D P}^{e}(x, t)}{\partial x^{2}}
$$

We then consider the transport of $\mathrm{BDP}^{2-}$ in the particle. Assuming there is only a migration contribution, the $\mathrm{BDP}^{2-}$ flux in the particle equals

$$
\boldsymbol{J}_{B D P}^{p}(0, t)=-\frac{\tau_{B D P}^{p} \boldsymbol{j}}{F}(1)
$$

We solve Equation 7.4 using the following boundary conditions

- At $x=\infty$, at any $t, \quad C_{B D P}^{e}(\infty, t)=C_{B D P}^{B}$

- $\quad$ At $t=0$, at any $x, \quad C_{B D P}^{e}(x, 0)=C_{B D P}^{B}$

- At $x=0$, at $t \neq 0, \quad \frac{\partial C_{B D P}^{e}(0, t)}{\partial x}=-\frac{j\left(\tau_{B D P}^{p}-\tau_{B D P}^{e}\right)}{F D}$

Here $x=0$ is the location of the particle/solution interface at the cathodic side of the particle and $x=\infty$ is a sufficient distance away from this point where there is no effect of the particle hence the $\mathrm{BDP}^{2-}$ concentration retains its initial bulk value $\left(C_{B D P}^{B}\right)$. Equation 7.8 simply expresses the conservation of mass for the fluxes crossing the plane at $x=0$. The result is, ${ }^{22}$ 


$$
C_{B D P}^{e}(x, t)=C_{B D P}^{B}-\frac{j\left(\tau_{B D P}^{p}-\tau_{B D P}^{e}\right)}{F D}\left\{2\left(\frac{D t}{\pi}\right)^{\frac{1}{2}} \exp \left(-\frac{x^{2}}{4 D t}\right)-x \operatorname{erfc}\left(\frac{x}{2(D t)^{\frac{1}{2}}}\right)\right\},
$$

where erfc is the complementary error function. In Equation 7.9 the variables,

- Current density $(\boldsymbol{j})$

- Transport numbers $\left(\tau_{B D P}^{e}, \tau_{B D P}^{p}\right)$,

depend on the electrolyte bulk salt concentration and the particle pore size, and we will investigate them separately in the following subsections.

\subsubsection{Transport number $\tau$}

The difference of $\mathrm{BDP}^{2-}$ transport numbers between electrolyte and particle $\left(\tau_{B D P}^{p}-\tau_{B D P}^{e}\right)$ indicates the ion permselectivity of the particle towards $\mathrm{BDP}^{2-}$. We thus will derive the transport numbers of $\mathrm{BDP}^{2-}$ in the electrolyte and in the particle. The ion transport number $\left(\tau_{i}\right)$ of a specie $i$ is defined as the fraction of the total electric current density $(j)$ carried by this specie. In the electrolyte the transport number for $\mathrm{BDP}^{2}$ - equals

$$
\tau_{B D P}^{e}=\frac{2 c_{B D P}^{e} D_{B D P}}{\sum\left|z_{i}\right| c_{i}^{e} D_{i}}
$$

In the particle we use the same equation but now with the area-averaged concentration of $\mathrm{BDP}^{-2}$ in the mesopore

$$
\tau_{B D P}^{p}=\frac{2 c_{B D P}^{p} D_{B D P}}{\sum\left|z_{i}\right| c_{i}^{p} D_{i}}
$$

In order to calculate the concentration in the pore we must account for the concentration distribution of the species in the electric double layer. We will follow the approach of Rice and Whitehead ${ }^{23}$ who solved the PoissonBoltzmann equations for a circular mesopore. Starting from the 1D Poisson equation for radial coordinates,

$$
\frac{\partial^{2} \psi}{\partial r^{2}}=-\frac{4 \pi}{\varepsilon_{0} \varepsilon_{r}} \rho(r)
$$

where $\psi[\mathrm{V}]$ is the local electric potential, $\mathrm{r}[\mathrm{m}]$ is the distance from the pore center, $\varepsilon_{0}$ and $\varepsilon_{r}$ are the vacuum and medium permittivity and $\rho\left[C / \mathrm{m}^{3}\right]$ is the local electric space charge density which is defined as,

$$
\rho(r)=\sum e z_{i} c_{i}(r)
$$


The concentration of the ions follows the Boltzmann distribution,

$$
c_{i}(r)=c_{i}^{p} \exp \left(-\frac{z_{i} e \psi(r)}{k_{B} T}\right)
$$

where $c_{i}^{p}\left[\mathrm{~mol} / \mathrm{m}^{3}\right]$ is the pore electrolyte concentration of species $i, e[C]$ the electron charge, $K_{B}$ the Boltzmann constant and $T[K]$ the absolute temperature. It is worth mentioning that for $c_{i}^{p}$ the bulk concentration of the species is used which doesn't hold for overlapping double layers. Hence, at low concentrations the results should be taken tentatively. For $\psi \leq k_{B} T / e$,

$$
\frac{1}{r} \frac{\partial}{\partial r}\left(r \frac{\partial \psi}{\partial r}\right)=\frac{\psi(r)}{\lambda_{D}}
$$

where $\lambda_{D}$ is the Debye length ${ }^{24,25}$.

$$
\lambda_{D}=\sqrt{\frac{\varepsilon_{0} \varepsilon_{r} R T}{2 F^{2} c^{e}}} .
$$

We use an approach similar to Taghipoor et $a .^{26}$ for the determination of the surface charge density $\left(\sigma\left[C / \mathrm{m}^{2}\right]\right)$. We then use the Grahame Equation 7.17, for the determination of surface potential $\left(\psi_{0}[V]\right)$ and equation 7.18 for the relation between the surface potential and zeta potential, the potential at the slipping plane.

$$
\begin{gathered}
\sigma=\sqrt{8 c_{i}^{p} N_{A} \varepsilon \varepsilon_{0} k_{b} T} \sinh \left(\frac{Z \varepsilon \psi_{0}}{2 k_{B} T}\right), \\
\psi_{0}-\zeta=\frac{\sigma}{C_{\text {stern }}}
\end{gathered}
$$

where $N_{A}\left[\mathrm{~mol}^{-1}\right]$ is the Avogadro number and $C_{\text {stern }}\left[F / \mathrm{m}^{2}\right]$ is the specific Stern layer capacitance. Equation 7.15 is then analytically solved for the potential distribution in the diffuse double layer ${ }^{27}$,

$$
\psi(r)=\zeta \frac{I_{0}\left(\frac{r}{\lambda_{D}}\right)}{I_{0}\left(\frac{r_{0}}{\lambda_{D}}\right)}
$$

where $I_{0}$ is the zero-order modified Bessel function of the first kind. From Equations 7.14 and 7.19 we can estimate the average BDP2- concentration inside 
the mesopore. We subsequently calculate the $\mathrm{BDP}^{2-}$ transport number in the mesopore (Equation 7.11) by integrating $C_{B D P}^{p}(r)$ over the pore cross-sectional area. Figure 7.2 shows the transport numbers of $\mathrm{BDP}^{2-}$ in the electrolyte and particle as a function of bulk electrolyte concentration using the average mesopore size determined by BET measurements (Table 7.1). At low electrolyte concentrations, the concentration of $\mathrm{BDP}^{2-}$ in the particle is extremely low compared to the total ion concentration (especially cation concentration) since the electric double layers overlap and $\mathrm{BDP}^{2-}$ is almost completely excluded from both small and large mesopores. At high electrolyte concentrations the electric double layer is extremely thin and the average species concentration is almost the same as the bulk concentration. The larger diameter pores approach this state at much lower electrolyte concentrations than the smaller pores.

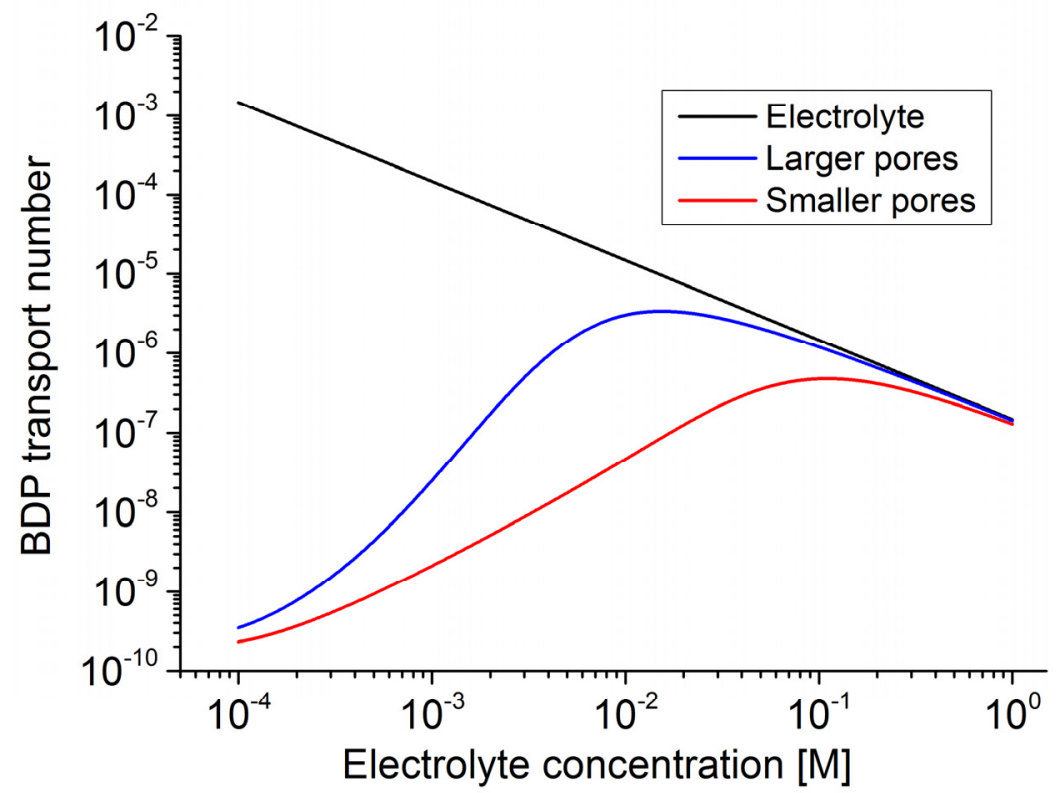

Figure 7.2. Transport number of BDP2- in the particle vs electrolyte concentration. Blacktransport number in the electrolyte, blue - in the larger pores and red-in the smaller pores.

\subsubsection{Current density $j$}

To determine the magnitude of this E-field, we need to consider our experimental system. In the CP model of sections 7.1 and 7.2, the porous particle was modelled as part of a continuous membrane. During the experiments however, separate particles are placed at the bottom of a microfluidic channel and a constant potential is applied at the two reservoirs connected to this channel. The E-field across the particle will then depend on the conductance ratio between particle and its surrounding electrolyte. Now when we vary the 
electrolyte concentration, the particle conductance scales differently from the electrolyte conductance, creating a dependency of the E-field across the particle on the electrolyte concentration. An equivalent 1D macroscopic electrical circuit is therefore needed to calculate the E-field as a function of concentration, and is shown in Figure 7.4a. From the total system conductance, the separated conductance of the electrolyte and the particle, and the applied voltage we can then calculate the potential drop over and hence the E-field across the particle.

We assume that a constant and uniform electric field $(E)$ exists inside the particle, i.e. the concentration distribution of the ions in the electrolyte close to the particle surface does not affect the local electric field inside the particle. The current density $(\boldsymbol{j})$ through the particle then equals

$$
\boldsymbol{j}=\boldsymbol{E} \kappa
$$

where $\kappa$ is the area-averaged conductivity of the particle. The area-averaged conductivity of a single mesopore $\left(\kappa_{c}\right)$ equals ${ }^{26}$

$$
\kappa_{c}=\frac{N_{A} e}{r_{0}} \sum_{i} \int_{0}^{r_{0}} \mu_{i} c_{i}(r) d r
$$

where $c_{i}(r)$ was calculated as in the previous section. The electric conductance $\left(G_{c}[S]\right)$ of a single mesopore then is

$$
G_{c}=\frac{A \kappa_{c}}{d}
$$

where $d[m]$ is the diameter of the particle (mesopore length) and $A\left[\mathrm{~m}^{2}\right]$ is the cross-sectional area of the mesopore. Figure 7.3 shows the conductivity of a single mesopore of the two kinds of particles, using the average mesopore size determined by BET measurements (Table 1). As expected, the smaller mesopore shows a higher conductivity.

We calculate the total conductance of a particle $\left(G_{p}\right)$ from the total number of mesopores $(N)$ per particle, treating them as parallel conductors

$$
G_{p}=N G_{c}
$$




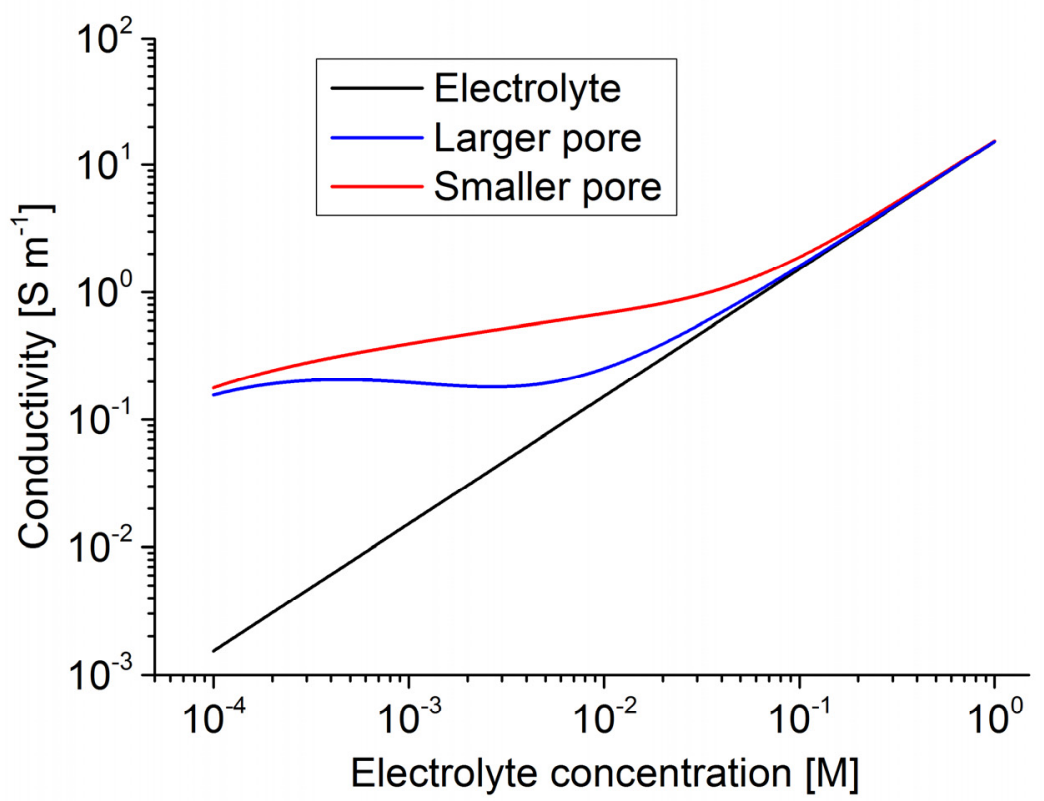

Figure 7.3. Single mesopore conductance vs salt concentration - Eq. 20-22. The surface charge density dependence to the concentration was taken from $M$. Taghipoor et a ${ }^{24}$. For high concentration where the double layer thickness is small compared to the mesopore width, the mesopore conductivity follows the bulk conductivity. In contrast at low concentrations the electric double layer thickness is similar to the channel diameter and the surface conductivity provides the dominant contribution.

For the calculation of $N$ we use the specific pore volume $\left(V_{s}\left[\mathrm{~m}^{3} / g\right]\right)$ or specific pore surface area $\left(A_{s}\left[\mathrm{~m}^{2} / \mathrm{g}\right]\right)$ and the density $\left(\rho\left[\mathrm{g} / \mathrm{m}^{3}\right]\right)$ values found via BET and pycnometer methods, as will be explained in the experimental section,

$$
N=\frac{\rho V_{\text {particle }} V_{s}}{V_{\text {pore }}}
$$

or

$$
N=\frac{\rho V_{\text {particle }} A_{s}}{A_{\text {pore }}}
$$

where $V_{\text {particle }}\left[\mathrm{m}^{3}\right]$ is the volume of the particle and $V_{\text {pore }}\left[\mathrm{m}^{3}\right]$ and $A_{\text {pore }}\left[\mathrm{m}^{2}\right]$ are the volume and surface area of a single mesopore.

Figure $7.4 \mathrm{~b}$ shows that the E-field remains relatively constant when the particle/pore conductivity is equal to the bulk conductivity. Once the bulk electrolyte concentration drops and the surface conductivity becomes the dominant conductivity contribution in the mesopore, the particle resistance increases slower than the resistance of the surrounding electrolyte resulting in 
a lower voltage drop and E-field across it. Knowing the conductivity and the Efield as a function of bulk concentration and mesopore size we can calculate the current density via Equation 7.20.

(a)

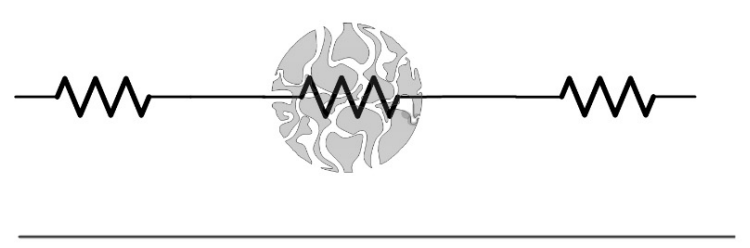

(b)

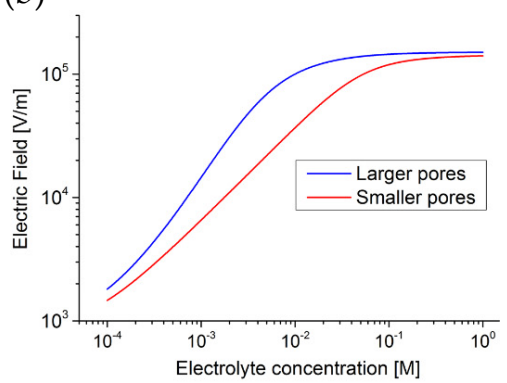

Figure 7.4. (a) Equivalent circuit to calculate the electric field across the particle with varying electrolyte concentration. (b) At high concentrations the particle resistance is high compared to the electrolyte resulting in an E-field across the particle that is weakly dependent of the concentration. At low concentration the particle resistance will increase slower compared to the bulk resistance resulting in a lower E-field in the particle.

Once the current density and transports numbers $\left(\tau_{B D P}^{p}-\tau_{B D P}^{e}\right)$ are determined, Equation 7.9 can be used to describe the temporal concentration profile of the fluorescent reporter, $\mathrm{BDP}^{2-}$, as a function of distance from the surface of the particle $\left(C_{B D P}(x, t)\right)$ in the enrichment zone. Figure 7.5 a shows the calculated average $\mathrm{BDP}^{2-}$ concentration of both particles over a distance of 25 $\mu \mathrm{m}$ in the bulk electrolyte over 4 seconds and at the edge of the particle (see Figure 7.5b). The shape of the graph is dominated by the value of the product $j\left(\tau_{B D P}^{p}-\tau_{B D P}^{e}\right)$ in Equation 7.9, representing the particle ion-permselectivity. At low concentrations, the cation permselectivity is maximal, hence the accumulation is governed by the conductance of the mesopores, with the larger pores being more conductive because of the larger pore volume. However, these pores lose their selectivity faster compared to the smaller pores as the bulk salt concentration increases. The two lines therefore cross, in the model at a bulk electrolyte concentration of $14 \mathrm{mM}$. In addition, at low concentrations the resistance of the particle is approximately two orders of magnitude lower than the resistance of the channel. Hence the resistance of the microchannel (Figure 7.4a) regulates the current through the system and particle resulting in the flattening of the $\mathrm{BDP}^{2-}$ concentration at low concentration at Figure 7.5a. 
(a)

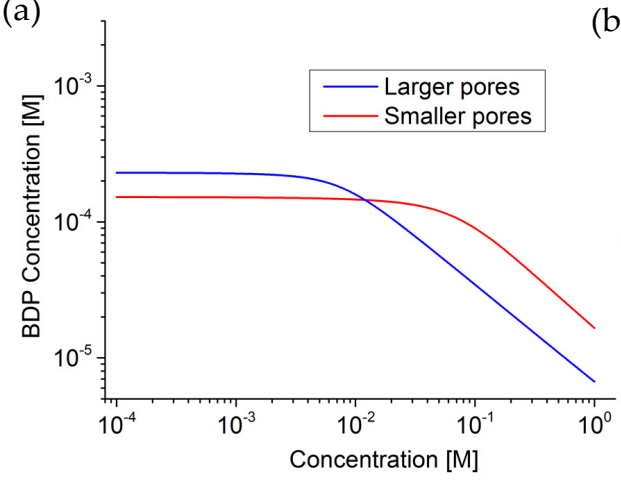

(b)

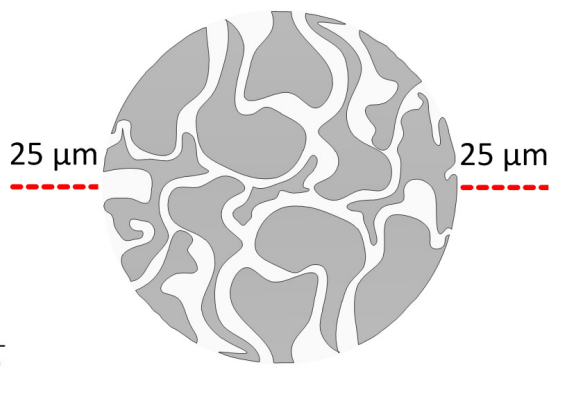

Figure 7.5. (a) Averaged BDP2- concentration at different electrolyte concentrations on the enrichment side of the particle over $4 \mathrm{sec}$ over $25 \mu \mathrm{m}$ from the particlelelectrolyte interface (as shown in b).

\subsection{Materials and methods}

A PDMS microfluidic chip was fabricated by standard photolithographic techniques. First, a $200 \mu \mathrm{m}$ SU8 layer was spun and baked on a 4-inch silicon wafer. Next, the photoresist was exposed for 50 seconds to UV light and developed. The chip design consisted of a straight channel $200 \mu \mathrm{m}$ height, $1 \mathrm{~mm}$ wide and $2 \mathrm{~cm}$ long, connecting two reservoirs. Polystyrene particles $50 \mu \mathrm{m}$ in diameter with two meso-pore sizes (10 and $50 \mathrm{~nm}$ ) were purchased at Chromspheres.com. Particles were submerged in ethanol and, with a pipette, they were deposited one by one on the PDMS channel. Thereafter, the PDMS with the particles were plasma treated for 40 seconds and bonded to a glass slide. The polystyrene particles were therefore stuck to the PDMS and sufficiently spaced between them in order to avoid interaction of the fluorescent reporter. A microscope Olympus IX51 and camera Grasshopper 3 were used with a $\mathrm{Hg}$ lamp for the fluorescent microscopy. Two platinum wires were used to connect the power supply (Keithley 2410) to the electrolyte solution at the reservoirs. BODIPY ${ }^{\mathrm{TM}} 492 / 515$ Disulfonate (BDP2-) (ThermoFisher) and phosphate buffered saline solution (PBS)(Sigma-Aldrich) were used as a fluorescent molecule and background electrolyte respectively. The PBS concentrations used were $10^{-4}, 10^{-3}, 10^{-2}, 2.5^{*} 10^{-2}, 5.0^{*} 10^{-2}, 7.5^{*} 10^{-2}$ and $10^{-1} \mathrm{M}$ while the concentration of $\mathrm{BDP}^{2-}$ was $750 \mathrm{nM}$ in all experiments. Matlab and ImageJ were used to analyse the experimental data. Matlab was used to simulate the model. The power supply was operated via LabView software. BET and density measurements were performed in a Gemini VII from Micromeritics and AccuPyc II 1340 Micromeritics pycnometer, respectively. The ICP was measured by applying a step function of $\pm 100 \mathrm{~V}$ across the channel, and performing 4 cycles of 4 seconds each. During the experimental data analysis, a 
linear drop of fluorescence intensity in the bulk, which we assumed to be caused by the marker photobleaching, was observed and compensated for.

\subsection{Results and discussion}

Figure 7.6 shows SEM images of both types of polystyrene particles showing a clear difference in pore size distribution. To measure the average pore size as well as the pore volume, surface area and density, BET and pycnometer measurements were performed, with the results summarized in Table 7.1. A clear difference between both types of particles can be observed, with both having pores in the mesoporosity size range. Also, the similar pore volume indicates that the particles with smaller pores have more pores per unit volume.

Figure 7.7a shows an image of a typical concentration polarization measurement of a particle with an accumulation zone on the cathodic side and a depletion zone on the anodic side. The area- and time-averaged fluorescence intensity at both sides of a particle was measured as illustrated in Figure 7.7a. Figure $7.7 \mathrm{~b}$ shows a typical experimental fluorescent intensity obtained in one positive and negative pulse.
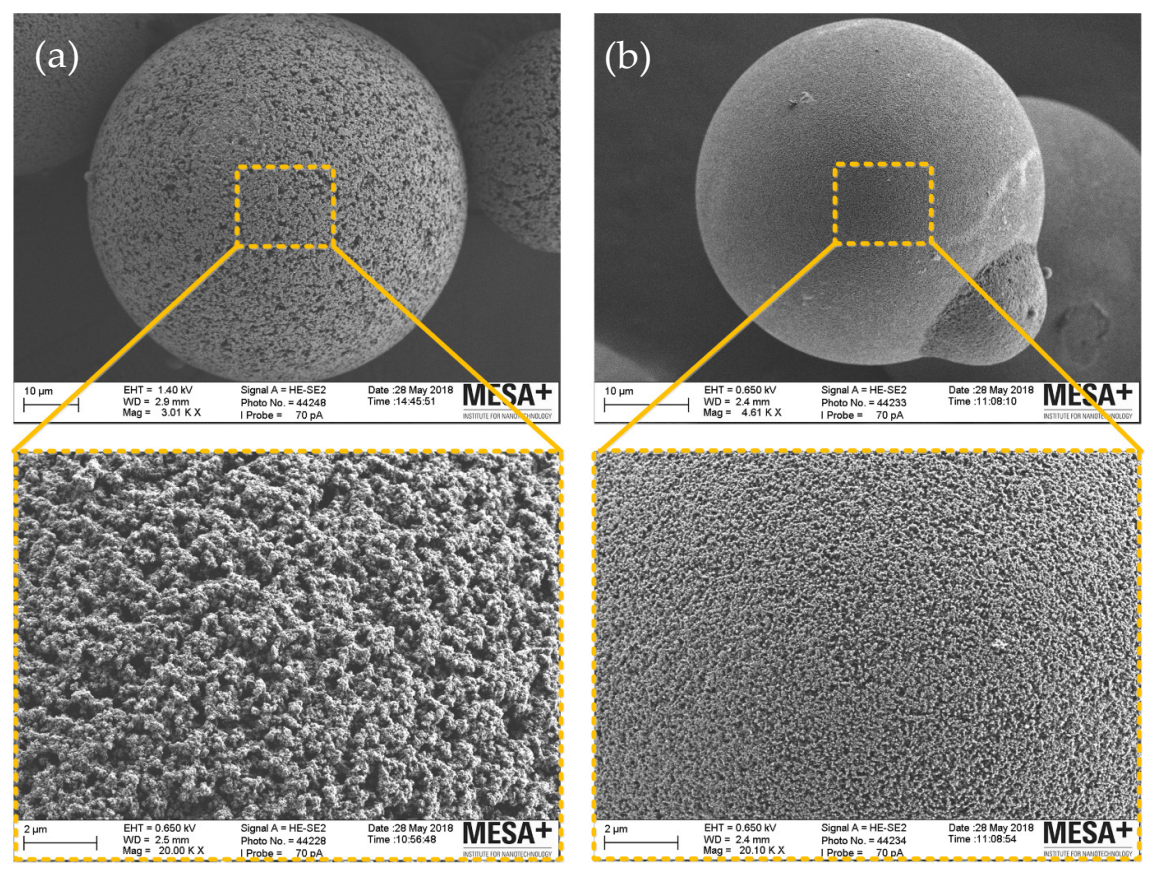

Figure 7.6. SEM images of polystyrene particles with (a) larger and (b) smaller pores. 
Table 7.1. Average pore radius, pore volume, surface area and density of both types of polystyrene particles.

\begin{tabular}{|c|c|c|c|}
\hline Property & Method & Larger pores & Smaller pores \\
\hline Average pore radius (r) & BET & $13 \mathrm{~nm}$ & $3.5 \mathrm{~nm}$ \\
\hline Total pore volume $(\boldsymbol{V})$ & BET & $1.910^{-6} \mathrm{~m}^{3} / \mathrm{g}$ & $1.510^{-6} \mathrm{~m}^{3} / \mathrm{g}$ \\
\hline Surface area $(A \boldsymbol{s})$ & BET & $39.8 \mathrm{~m}^{2} / \mathrm{g}$ & $158.59 \mathrm{~m}^{2} / \mathrm{g}$ \\
\hline Density $(\boldsymbol{\rho})$ & Pycnometer & $1.100 \mathrm{~g} / \mathrm{cm}^{3}$ & $1.100 \mathrm{~g} / \mathrm{cm}^{3}$ \\
\hline
\end{tabular}

(a)

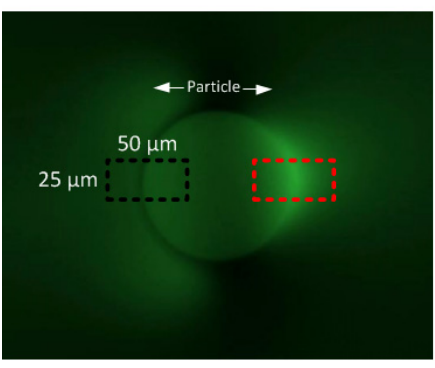

(b)

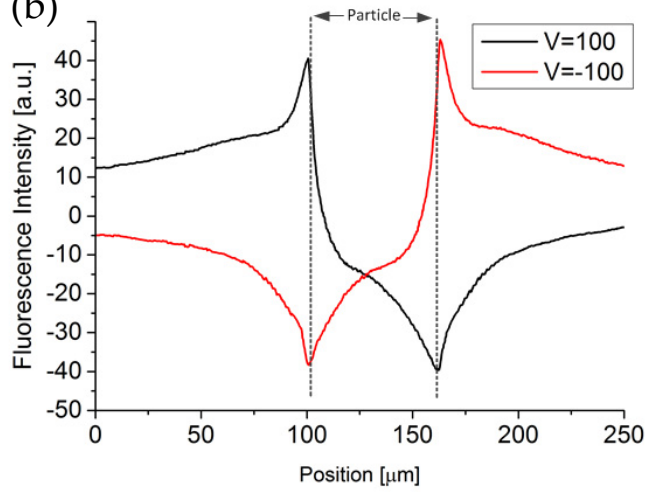

Figure 7.7. (a) Image of a typical measurement showing an accumulation zone on the right and the areas that were measured and averaged over 4 seconds. (b) a typical fluorescent intensity profile for a positive and negative potential pulse.

The experimentally determined difference in fluorescence intensity between depletion and enrichment zones is plotted against the electrolyte concentration in Figure 7.8. It was found that the particles with larger pores have a higher fluorescence intensity at low salt concentrations. This was indeed predicted by our model, where it results from the larger pore volume. The fluorescence intensity values of both particles cross at a higher salt concentration, which was also predicted in the model, where it follows from the earlier drop in the ion permselectivity of the particles with larger pores. This confirms that ICP can be used to differentiate between particles with different mesoporosity. The error bars in Figure 7.8 correspond to $95 \%$ confidence intervals and are a result of various factors. The particle size varied significantly $(106,7 \mu \mathrm{m} \pm 16,8 \mu \mathrm{m})$, resulting in strong variations in pore conductance. Furthermore, the average pore size, pore volume and surface area were measured in a sample with a large number of particles and the variation between particles is unknown. The differences between the model results and the experimental can furthermore result from the various assumptions made in the model. Specifically, the electroosmotic flow is neglected and the pores are 
assumed to be straight and cylindrical, while in reality a network of connected pores spaces exists as can be expected from the SEM images in Figure 7.6.

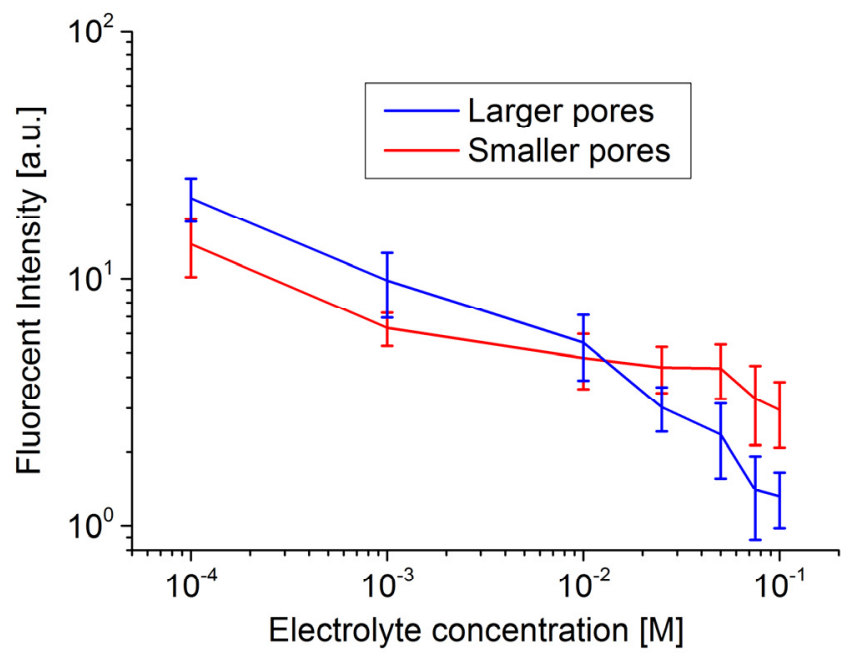

Figure 7.8. Experimental results of ion concentration polarization vs electrolyte concentration. The points on y-axis were found by subtracting the lower fluorescent intensity value (in the depletion zone) from the high intensity value (in the enrichment zone). Similar to the theoretical graph, the ICP at low electrolyte concentration is larger for the large pore diameter particles, while the ICP at high salt concentration is larger for the small pore diameter particles. The lines cross in both the model and the experiments at a concentration where the selectivity is lost for the larger pores at lower concentrations. The error bars correspond to 95\% confidence intervals.

As some experimental verification of the model is now obtained, we will investigate its feasibility for future applications by modelling the effects of the following properties on the ICP: pore diameter, surface charge density, and total particle surface area. Figure 7.9a shows that an increase in pore size causes a faster decrease in fluorescent intensity with increasing electrolyte concentration which can be explained by the fact that smaller pores will be more permselective than larger pores at higher electrolyte concentrations, causing a higher transport number difference with the bulk electrolyte. It is worth noting that the total particle cross-sectional area was kept constant hence a constant current density is assumed on Figure 7.9a. In the case of varying surface charge, see Figure $7.9 \mathrm{~b}$, at low electrolyte concentration no difference is observed, that is caused by the limitation of the current due high resistance of the bulk compared to the particle as commented earlier. In contrast a difference is seen when the electrolyte concentration increases, the pores with high surface charge lose their permselectivity at higher electrolyte concentrations due to higher concentration in the double layer. Figure 7.9c shows the effect of total particle surface area on the reporter concentration. As can be seen, a lower surface area causes an increase in fluorescent intensity. The total surface area is linearly (for 
a constant pore size) related to the number of pores. Since the total current through the particle is regulated by bulk resistance (microchannel) it is almost constant between particles. A decrease in the surface area translates in lower number of pores which results in a higher current density through the particle. The conclusion is that differences in pore size and pore charge density lead to different concentration polarization behaviour. In addition to a qualitative description of our system, also more specific information can be extracted from our model. As can be seen in Figure 7.9a, the cut-off point in the electrolyte concentration (intercept of red lines) where the concentration polarization effect starts to drop when increasing the electrolyte concentration is strongly correlated to the pore size as well as the surface area, with the effect of pore size being larger. On the other hand, the concentration polarization at very low electrolyte concentrations is dominated just by the specific surface area of the particle (Figure 7.9c), hence by measuring the ICP at low electrolyte concentrations $\left(10^{-4}[\mathrm{M}]\right)$ the particles can be differentiated based on their specific surface area. As can be seen in the experimental data of Figure 7.8, the fluorescent intensity at low concentrations is slightly lower for the particle with smaller pores, indicating that their specific surface area is slightly larger. However, when evaluating the concentration at which the fluorescent intensity drops, a parameter that is largely dependent on the pore size, the ICP of the particles with larger pores drops at lower concentrations as predicted by our model.

\subsection{Conclusions}

In conclusion, we demonstrated that ICP can be used to differentiate between particles with different meso-pore dimensions. A model was developed demonstrating that both pore density and ion permselectivity play a role in the magnitude of ICP. Subsequently, the ICP of two types of particles with experimentally determined porosity and pore density was measured at different bulk electrolyte concentrations. Experimental and simulated results show similar trends. We conclude that the ICP method can be used to track differences in pore size between mesoporous particles such as catalysts or catalysts supports. 

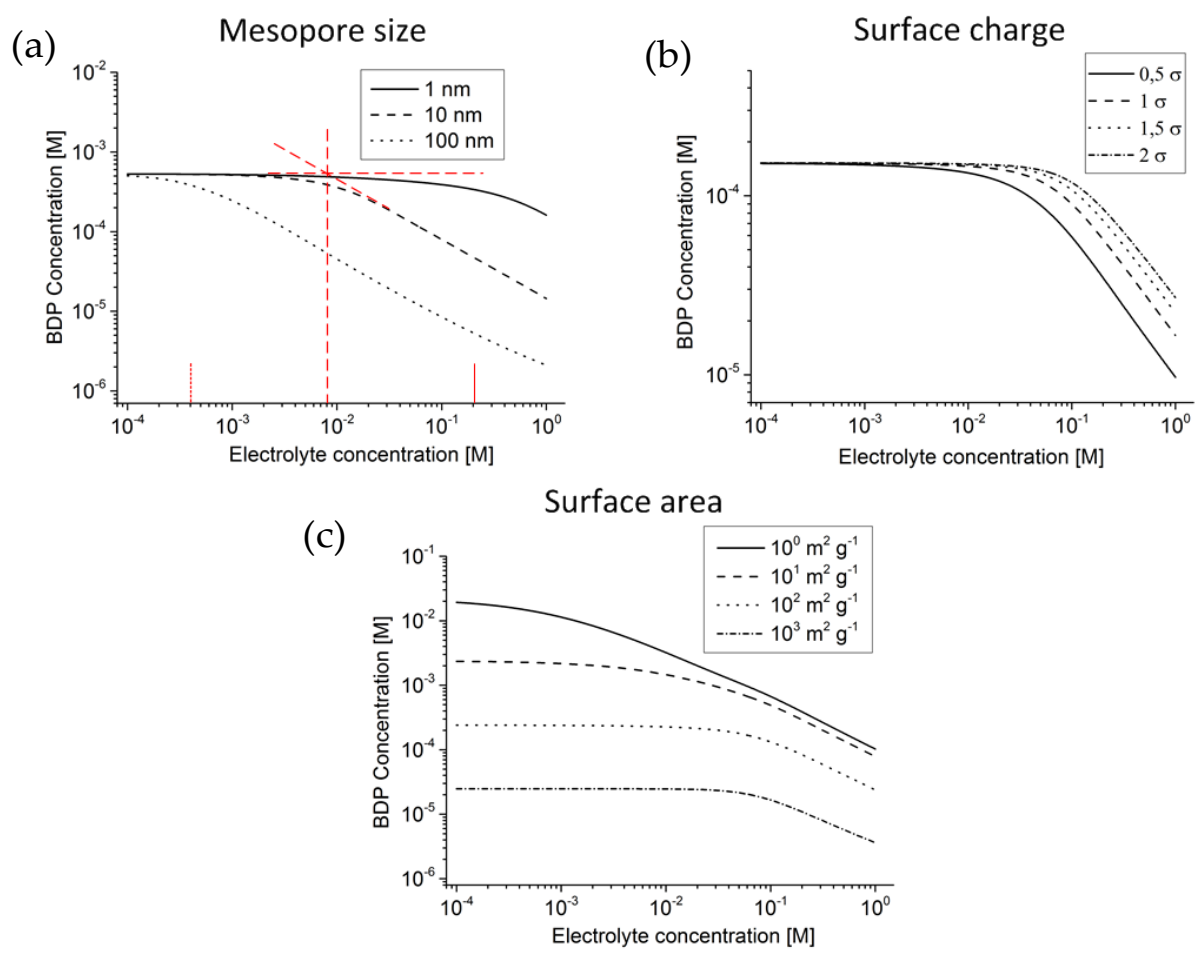

Figure 7.9. Simulations of the model when changing (a) the mesopore size, (b) surface charge density and (c) surface area. All the other parameters are kept the same as the smaller pores particle. $\sigma$ corresponds to the surface charged density calculated for glass as described previously.

\subsection{References}

1. Kitagawa, S. Porous Materials and the Age of Gas. Angew. Chemie - Int. Ed. 54, 10686-10687 (2015).

2. Zaworotko, M. J. Designer pores made easy. Nature 451, 410-411 (2008).

3. Davis, M. E. Ordered Porous Materials for Emerging Applications. Nature 10, 813-821 (2015).

4. Parlett, C. M. A., Wilson, K. \& Lee, A. F. Hierarchical porous materials: Catalytic applications. Chem. Soc. Rev. 42, 3876-3893 (2013).

5. De Winter, D. A. M., Meirer, F. \& Weckhuysen, B. M. FIB-SEM Tomography Probes the Mesoscale Pore Space of an Individual Catalytic Cracking Particle. ACS Catal. 6, 3158-3167 (2016).

6. Wise, A. M. et al. Nanoscale Chemical Imaging of an Individual Catalyst Particle with Soft X-ray Ptychography. ACS Catal. 6, 2178-2181 (2016).

7. Meirer, F. et al. Life and death of a single catalytic cracking particle. Sci. Adv. 1, e1400199 (2015).

8. Cychosz, K. A., Guillet-Nicolas, R., García-Martínez, J. \& Thommes, M. Recent advances in the textural characterization of hierarchically structured nanoporous materials. Chem. Soc. Rev. 46, 389-414 (2017). 
9. Meirer, F. \& Weckhuysen, B. M. Spatial and temporal exploration of heterogeneous catalysts with synchrotron radiation. Nat. Rev. Mater. 324-340 (2018).

10. Ning, G., Wei, F., Luo, G. \& Jin, Y. Online BET analysis of single-wall carbon nanotube growth and its effect on catalyst reactivation. Carbon $N$. Y. 43, 1439-1444 (2005).

11. Leofanti, G., Padovan, M., Tozzola, G. \& Venturelli, B. Surface area and pore texture of catalysts. Catal. Today 41, 207-219 (1998).

12. Ihli, J. et al. A three-dimensional view of structural changes caused by deactivation of fluid catalytic cracking catalysts. Nat. Commun. 8:809, (2017).

13. Gregg, S. J. \& Sing, K. S. W. Adsorption, Surface Area and Porosity. (Academic Press, 1982).

14. Rouquerol, J. et al. Liquid intrusion and alternative methods for the characterization of macroporous materials (IUPAC Technical Report). Pure Appl. Chem. 84, 107-136 (2011).

15. Liu, Y., Meirer, F., Krest, C. M., Webb, S. \& Weckhuysen, B. M. Relating structure and composition with accessibility of a single catalyst particle using correlative 3-dimensional micro-spectroscopy. Nat. Commun. 7:12634, (2016).

16. Whiting, G. T., Nikolopoulos, N., Nikolopoulos, I., Chowdhury, A. D. \& Weckhuysen, B. M. Visualizing pore architecture and molecular transport boundaries in catalyst bodies with fluorescent nanoprobes. Nat. Chem. 11, 23-31, (2019).

17. Kim, S. J., Li, L. D. \& Han, J. Amplified electrokinetic response by concentration polarization near nanofluidic channel. Langmuir 25, 7759 7765 (2009).

18. Plecis, A., Clément, N., Haghiri-Gosnet, A.-M. \& Chen, Y. Electropreconcentration with Charge-Selective Nanochannels. Anal. Chem. 80, 9542-9550 (2008).

19. Pu, Q., Yun, J., Temkin, H. \& Liu, S. Ion-Enrichment and Ion-Depletion Effect of Nanochannel Structures. Nano Lett. 4, 1099-1103 (2004).

20. Petr Vanysek. Ionic conductivity and diffusion at infinite dilution. CRC Handbook of Chemistry and Physics, 91th Editio (1991).

21. Bergveld, P., Eijkel, J. C. T. \& Olthuis, W. Detection of protein concentrations with chronopotentiometry. Biosens. Bioelectron. 12, 905916 (1997).

22. Bard, A. J. et al. ELECTROCHEMICAL METHODS Fundamentals and Applications. Faulkner, L.R. Bard A.J. (1944).

23. Rice, C. L. \& Whitehead, R. Electrokinetic flow in a narrow cylindrical capillary. J. Phys. Chem. (1965).

24. El-Gholabzouri, O., Cabrerizo-Vílchez, M. Á. \& Hidalgo-Álvarez, R. Zeta-potential of polystyrene latex determined using different 
electrokinetic techniques in binary liquid mixtures. Colloids Surfaces $A$ Physicochem. Eng. Asp. 291, 30-37 (2006).

25. Plecis, A., Schoch, R. B. \& Renaud, P. Ionic transport phenomena in nanofluidics: Experimental and theoretical study of the exclusionenrichment effect on a chip. Nano Lett. 5, 1147-1155 (2005).

26. Taghipoor, M., Bertsch, A. \& Renaud, P. An improved model for predicting electrical conductance in nanochannels. Phys. Chem. Chem. Phys. 17, 4160-4167 (2015).

27. Hunter, R. J. Zeta Potential in Colloid Science. Microscopy (1981). 



\title{
Chapter
}

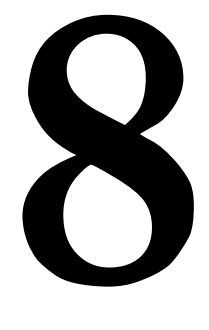

\section{Supplementary Results: Single Catalyst Particle Reactor}

\begin{abstract}
A ctivity characterization of catalysts particles is normally done in large vessels providing an ensemble average. The spread in activity of single particles within this ensemble may be quite large. However, single particle analysis requires expensive technology. Therefore, new tools to analyse catalyst particles at single particle level are needed. Here, we present a single particle reactor that can operate at high temperatures. We introduced a fluid catalytic cracking particle and tested its activity with n-hexane. During the n-hexane cracking, an increasing fluorescence indicated carbon formation on the fluid catalytic cracking particle. Carbon is a typical by-product when hydrocarbons such as nhexane are cracked. Also, a decreasing fluorescence was observed when the fluid catalytic cracking particle was fed with $\mathrm{O}_{2}$, which indicates carbon removal from the catalyst.
\end{abstract}




\subsection{Introduction}

In a world that is struggling with climate change, alternative and sustainable fuels such as: carbon dioxide, water or biomass, have emerged as possible options to supply energy. However, this requires new challenges to break and create bonds in a controllable manner. Catalysis, in particular heterogeneous catalysis, uses solid materials to convert gas or liquid reagents into products in a more efficient manner. ${ }^{1}$ Normally, these catalysts are produced in batch reactors with lack of control on their individual morphology and composition which results in intra and inter-particle heterogeneities.

The activity characterization of catalyst particles, an important step towards a better understanding of their performance, is always done in reactors containing thousands or millions of particles, called packed-bed reactors, which provide an ensemble average..$^{2,3}$ As a result, there is little knowledge of the individual performance and understanding of each catalytic particle. In order to characterize them at single particle level, spectroscopic techniques ${ }^{4-9}$ are used such as: scanning electron microscopy, x-ray spectroscopy, electron probe microanalysis and atomic force microscopy. However, these techniques are elaborate and/or expensive. Lately, some analytical tools have been developed to analyse the activity of catalyst particles at single particle level. These methods use fluorescent molecules that bond to the active sides of acid catalysts. ${ }^{10-13}$ Although these methods show a clear relation to the activity of these catalysts, yet there is a need to develop new tools to directly characterize the activity of catalysts particles at the single particle level.

In this chapter we developed a single particle reactor to measure the activity of single catalyst particles. A catalytic system suitable for this purpose is the fluid catalytic cracking (FCC) catalyst. ${ }^{14}$ FCC particles are $50-150 \mu \mathrm{m}$ big porous and solid acid catalysts, responsible for cracking the long chain hydrocarbons into smaller and more useful molecules in the crude oil industry. Previous work has demonstrated the use of n-hexane to prove the activity of catalysts containing zeolites such as FCC particles in a reactor, however this was done in bulk conditions. ${ }^{15}$ FCC particles crack hexane into smaller molecules and as a by-product carbon is produced and deposited on the catalysts. Here we report the fabrication of a single catalyst particle chip to test the carbon formation of FCC particles from h-hexane by fluorescent microscopy.

\subsection{Materials and methods}

The micro-reactor was fabricated using standard photolithographic techniques, see Sections E.1 and E.2 in Appendix. In short, straight channels were etched in two glass wafers and they were bonded and diced, see Figure 8.1. Each chip was $7 \mathrm{~cm}$ long and 1,5 cm wide, containing 4 microreactors per chip in order to increase the amount of products produced during the same experiment. FCC particles were introduced inside the chips by placing them 
close to the entry of the channel and using DI water, capillary forces and gravity to push them to the middle of the chip, see Figure 8.2. Fused silica capillaries were introduced in the channels and glued with Araldite adhesive epoxy 400007.

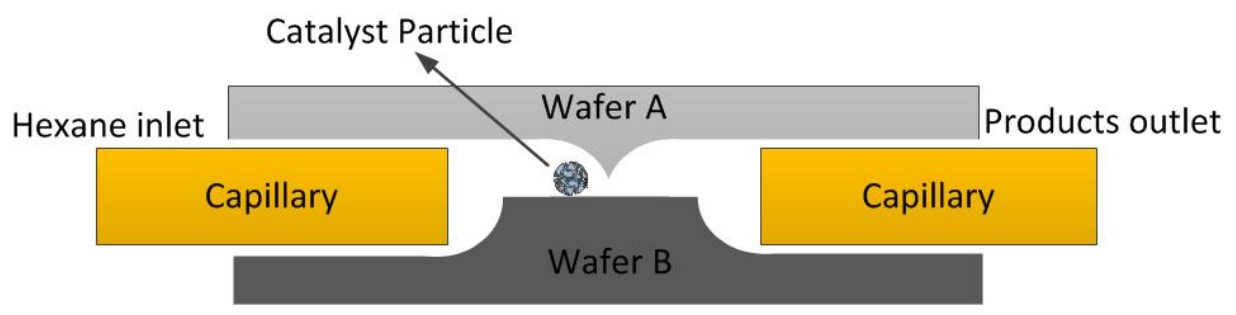

Figure 8.1. Side-view schematic drawing of a single particle microreactor.

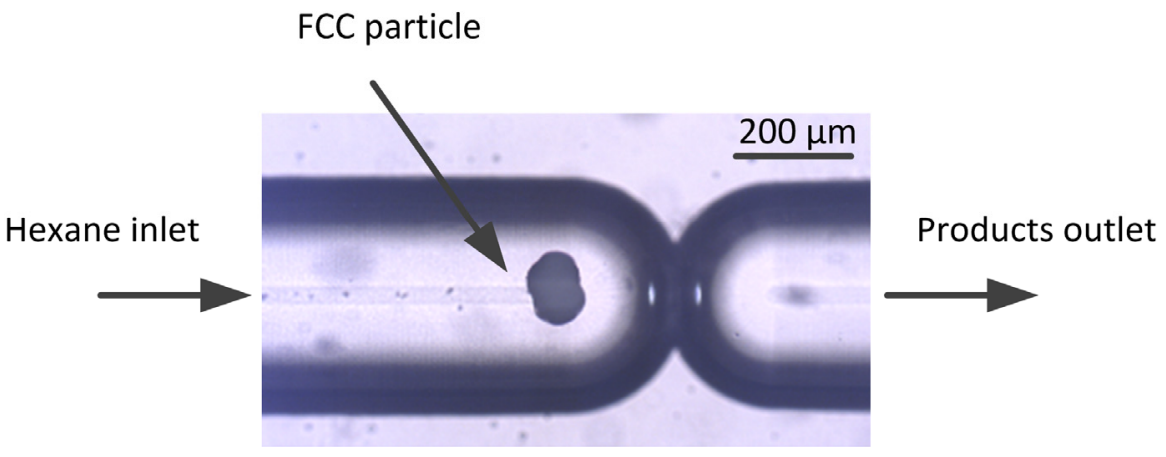

Figure 8.2. Top-view image of a particle inside the microreactor.

During the measurements, the chip containing 4 FCC particles was placed on the stage of a Linkam cell for heating, see Figure E.3 in Appendix. The fused silica capillaries were connected via T-junctions to one $\mathrm{N}_{2}$ gas and to liquid nhexane via a $1 \mathrm{~mL}$ glass Hamilton syringe. The Linkam cell was heated up to $400{ }^{\circ} \mathrm{C}$. It is expected that the final temperature of the microreactor is lower than the actual temperature set on the Linkam cell. A $\mathrm{N}_{2}$-flow of $4 \mathrm{~mL} / \mathrm{min}$ was applied to the system. This flow was split over all 4 particles and therefore, per particle a flow of $1 \mathrm{~mL} / \mathrm{min}$ is expected. After heating the particles in $\mathrm{N}_{2}$, the syringe with $\mathrm{n}$-hexane was connected with a flow $0.16 \mu \mathrm{L} / \mathrm{min}$. Upon heating, it is expected that the hexane is gasified immediately before reaching the FCC particle. The particles were visualized with a Nikon Eclipse 90i confocal fluorescence microscope with a Nikon-Eclipse A1R scan head (20x objective) equipped with a $561 \mathrm{~nm}$ solid state Sapphire laser, see Figure E.3 in Appendix. 


\subsection{Results and discussion}

\subsubsection{Analysis of the carbon formation and removal of a single FCC particle}

During the heat treatment and n-hexane experiments one of the particles was followed with fluorescence microscopy. Interestingly, during the supply of $\mathrm{n}$-hexane in $\mathrm{N}_{2}$-flow, the fluorescence signal increased, see Figure 8.3, while after the switch to a $\mathrm{O}_{2}$, the fluorescence decreased, see Figure 8.4. This would indicate that coke species are formed when flowing n-hexane on the FCC particle in the microreactor which verifies that $\mathrm{n}$-hexane was being cracked by the catalyst.
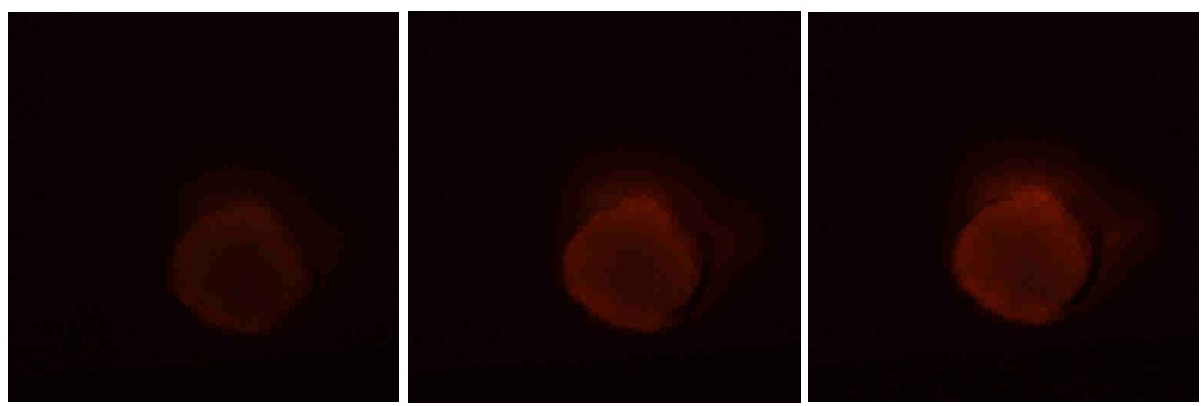

Figure 8.3 Fluorescence images of 1 particle during hexane flow. From left to right, 0 min, 5 min, $20 \mathrm{~min}$
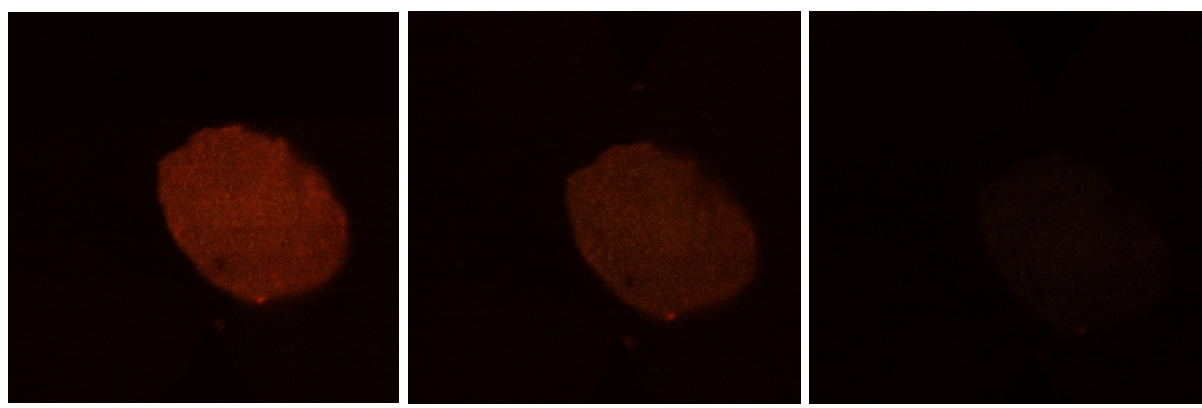

Figure 8.4. Fluorescence images of 1 particle during calcination in an oxygen flow. From left to right, $0 \mathrm{~min}, 10 \mathrm{~min}, 30 \mathrm{~min}$

\subsection{Conclusions}

In conclusion, a single particle reactor was successfully fabricated and nhexane and $\mathrm{O}_{2}$ were supplied inside the reactor with single FCC particles. During the experiments, an increasing fluorescence on the particles was observed provably caused by coke formed during n-hexane cracking. Decreasing fluorescence was observed when n-hexane was replaced with $\mathrm{O}_{2}$ indicating that the coke was being removed. 


\subsection{References}

1. Ertl, G., Knözinger, H., Schöth, F. \& Weitkamp, J. Handbook of Heterogeneous Catalysis, 8 Volumes, 2nd Edition. (2008).

2. Doraiswamy, L. K. \& Tajbl, D. G. LABORATORY CATALYTIC REACTORS. Catal. Rev. Sci. Eng. 36, i-ii (1994).

3. Munirathinam, R., Huskens, J. \& Verboom, W. Supported catalysis in continuous-flow microreactors. Adv. Synth. Catal. 357, 1093-1123 (2015).

4. Meirer, F. et al. Agglutination of single catalyst particles during fluid catalytic cracking as observed by X-ray nanotomography. Chem. Commun. 51, 8097-8100 (2015).

5. Meirer, F. et al. Life and death of a single catalytic cracking particle. Sci. Adv. 1, e1400199 (2015).

6. Wise, A. M. et al. Nanoscale Chemical Imaging of an Individual Catalyst Particle with Soft X-ray Ptychography. ACS Catal. 6, 2178-2181 (2016).

7. Ihli, J. et al. A three-dimensional view of structural changes caused by deactivation of fluid catalytic cracking catalysts. Nat. Commun. 8, 809 (2017).

8. Ihli, J. et al. Localization and Speciation of Iron Impurities within a Deactivated Fluid Catalytic Cracking Catalyst. Angew. Chem. Int. Ed. 56, 1-6 (2017).

9. De Winter, D. A. M., Meirer, F. \& Weckhuysen, B. M. FIB-SEM Tomography Probes the Mesoscale Pore Space of an Individual Catalytic Cracking Particle. ACS Catal. 6, 3158-3167 (2016).

10. Buurmans, I. L. C. et al. Catalytic activity in individual cracking catalyst particles imaged throughout different life stages by selective staining. Nat. Chem. 3, 862-867 (2011).

11. Buurmans, I. L. C. et al. Staining of fluid-catalytic-cracking catalysts: Localising Brønsted acidity within a single catalyst particle. Chem. - A Eur. J. 18, 1094-1101 (2012).

12. Aramburo, L. R. et al. Styrene oligomerization as a molecular probe reaction for Brønsted acidity at the nanoscale. Phys. Chem. Chem. Phys. 14, 6967-6973 (2012).

13. Whiting, G. T., Nikolopoulos, N., Nikolopoulos, I., Chowdhury, A. D. \& Weckhuysen, B. M. Visualizing pore architecture and molecular transport boundaries in catalyst bodies with fluorescent nanoprobes. Nat. Chem. (2018).

14. Vogt, E. T. C. \& Weckhuysen, B. M. Fluid catalytic cracking: recent developments on the grand old lady of zeolite catalysis. Chem. Soc. Rev. 44, 7342-7370 (2015).

15. Goetze, J. Spectroscopic studies on the formation of coke on individual Fluid Catalytic Cracking particles: the effect of poisoning metal compounds. 


\section{Chapter}

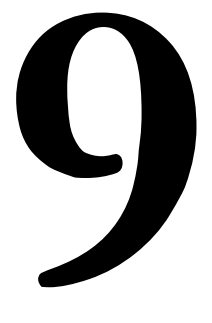

\section{Summary and Outlook}

he main results and conclusions are summarized in this chapter. In addition,
recommendations for further research are presented. 


\subsection{Summary}

Heterogeneous catalysts are solid materials that speed up and/or enable chemical reactions. However, due to their fabrication process, they end up having large inter and intra-particle heterogeneities. Typical activity and selectivity characterization of these materials is performed in batch reactors providing averaged experimental results. This hinders the knowledge of the individual role of each particle and decreases the understanding of the phenomena. On the other hand, single catalyst particle characterization uses expensive technology providing a low throughput. Hence, there is a need for inexpensive single particle characterization systems.

As stated in chapter 2, during the last couple of decades microfluidic technologies have evolved to sort and analyse single cells at high throughput. By using electric, magnetic, acoustic waves, or simply the inertia of fluids inside microsystems, the microfluidics community has developed many technologies to handle, sort and analyse microparticles and cells.

In this thesis, we employed some of the previously developed technologies, modified them or simply invented new ones to analyse catalyst particles at single particle level. With this approach, we could close the gap between single catalyst particle characterization and inexpensive technology and, where possible, at high throughput.

The catalyst case scenario that we used in some of the chapters was for fluid catalytic cracking (FCC) particles. FCC particles are porous, 50-150 $\mu \mathrm{m}$ in diameter catalyst particles responsible for cracking the long chain hydrocarbon molecules in the crude oil industry. Even though they have been extensively used during the last 80 years, they deactivate due to, amongst other processes, $\mathrm{Fe}, \mathrm{Ni}$ and $\mathrm{V}$ coming from the feedstock. In order to maintain the efficiency of the reactor, FCC particles are constantly replaced by fresh catalysts, resulting in a mixture called Equilibrium Catalyst (ECAT). Whilst Ni and V decrease the activity of the catalyst by enhancing other reactions, Fe is deposited on the first $2 \mu \mathrm{m}$ layer from the surface and it is supposed to block the pores, hence deactivating the particles. Knowing this, in chapter 3 we developed a magnetophoretic 3-D printed microfluidic chip able to sort ECAT FCC particles in 5 different fractions by their magnetic moment. Experimental results showed that their magnetic moment correlated with their Fe content. Also, zero-field cooled (ZFC) and field-cooled (FC) analysis demonstrated that Fe is deposited in small clusters a few nanometres big. Activity characterization showed an overall decreasing activity when increasing Fe content, confirming that Fe is one of the main deactivators of these particles. However, not all the particles with a high Fe content had a low activity. When looking at those particles, some black and orange spots were observed which were formed by Fe. These spots increased in size and number with increasing Fe content. A possible explanation 
for the no-relation between Fe content and activity in some FCC particles is the Fe agglomeration in spots which leaves the rest of the particle active.

In order to sort particles by Fe distribution, and thus improve the activity sorter previously described, we developed a magnetic particle spinner. By applying a rotating magnetic field, just the particles with an in-homogeneously distributed magnetic material experience a torque and therefore they rotate. Consequently, the rotation causes a perpendicular force to the translational movement of the particles called the Magnus force. Due to the lack of literature on this phenomena at the microscale, chapter 4 is focused on the demonstration of using this technique to sort microparticles by the Magnus force. The experimental results showed that the Magnus force can deflect the particles flowing through a channel, however, the deflection achieved is too small to be directly used as a sorting system.

When FCC particles get deactivated by metal accumulation, their dielectric properties also change. For most of the deposited metal oxides dielectric constant and conductivity are greater than those of the particles' main materials, silica and alumina. In biology, flow impedance cytometry measures the dielectric properties of cells at single cell level and high throughput. For this reason, in chapter 5 we developed a microfluidic chip to measure the dielectric properties of FCC particles at single particle level. Although we experimentally verified the system, no differences in dielectric properties were measured between FCC particles with different metal content.

In flow impedance cytometry, the position of the cell or particle between the electrodes is of great importance due to the inhomogeneity of the electric field, the so-called fringing effects. Hence, 2 identical particles flowing at 2 different positions between the electrodes can produce 2 different signals. However, this can be solved by detecting the position of the particle and correcting the final signal. Chapter 6 shows a new method to measure the position of microparticles between two electrodes. This is done by applying a non-uniform platinum black electrodeposited areas across both electrodes in a parallel electrodes array. By doing so, the particles' positions can be tracked at low frequencies $(800 \mathrm{~Hz})$ while their conductivity is measured at high frequencies $(100 \mathrm{kHz})$.

As stated previously, Fe accumulates in the first $2 \mu \mathrm{m}$ layers of FCC particles, and is thereby supposed to block the outer pores of the particles, hence preventing the reagents from getting inside and deactivating the particle. Porosity characterization is normally performed either in large vessels, therefore providing an ensemble average, or at single particle level using expensive technology and providing low throughput. Therefore, new methods are needed to measure particle porosity at single particle level. Ion concentration polarization (ICP) occurs at the interface of an ion-permselective material and free solution upon applying an electrical field. It results in the formation of ion accumulation and depletion zones at the opposite sides of the 
material due to the ion flux imbalance. Porous materials become ionpermselective when their pore dimensions are on the order of the electrical double layer thickness at the pore walls (typically nanometres). In chapter 7 we introduced 2 types of polystyrene particles with average nanopores of $3 \mathrm{~nm}$ and $13 \mathrm{~nm}$ in a simple PDMS microfluidic chip and used ICP to differentiate between them. We also developed a qualitative model able to predict the concentration of a fluorescent marker at the accumulation and depletion zones which was experimentally verified.

Catalyst activity and selectivity characterization is normally done in reactors with thousands to billions of particles, therefore providing an averaged result. To the best of our knowledge, direct single catalyst microparticle activity and selectivity characterization has never been done. In chapter 8 we developed a single catalyst microparticle reactor where single ECAT FCC particles were introduced. N-hexane was used as reagent and, during the catalytic process, a higher fluorescent intensity was observed, possibly related to an increased carbon content due to n-hexane cracking. In addition, the fluorescence decreased upon introduction of $\mathrm{O}_{2}$ in the reactors, possibly due to carbon combustion and removal.

\subsection{Outlook}

In this thesis, work has been presented regarding the separation and characterization of single catalyst particles.

Chapters 3 and 4 showed that microfluidic technologies are suitable for sorting catalyst particles. However, chapter 4 just demonstrated the possibility of rotating particles to study their composition. Future work should be focused on trying to increase the deflection of particles by, for example, increasing the magnetic field inside the fluidic chip and therefore the maximum rotation speed that the particles can follow. This technology could be applied to catalyst particles but also to other systems such as magnetic bacteria or cells labelled with superparamagnetic nanoparticles.

Chapters 5 and 7 used well-known phenomena to characterize catalyst particles. In the case of FCC particles, catalyst particle differentiation using electric fields needed to be supported by fresh particles that were very homogenous in composition. The small differences in dielectric constant between active and deactivated particles seemed to be lower or similar to those between active particles. Therefore, we think that future work should focus on either developing systems with a better sensitivity and/or particles more homogeneous in composition.

ICP is a promising technique to characterize catalyst particles. We believe that next steps should focus on quantifying the average meso-pore size of a single porous particle. This would result in the development of a new inexpensive technique able to measure the porosity of a microparticle which 
would facilitate the study of meso-porous materials. In addition, ICP could be applied in flow fashion which would enable single particle porosity study at high-throughput: definitely a promising technique to be applied in catalysis.

Last but not least, chapter 8 presented a new single particle reactor. Provided that this early stage work could be improved to analyse the outlet products, this system would be a promising method to directly study a single catalytic particle.

As stated in chapter 2, the microfluidics community has enabled the possibility of using new technology able to sort micrometre-sized particles at high throughput. Normally, heterogeneous catalyst particles are made of metal nanoparticles supported in porous oxide microparticles. However, usually the deposition of the nanoparticles is not homogeneous throughout the microparticle. Therefore, sorting the microparticles at single particle level for example, by magnetic or electric fields, would provide results dependent on the starting composition and distribution, complicating the study of the catalytic properties. This could be solved by pre-sorting the catalyst particles prior to catalysis or by synthesising identical microparticles with identical amount and distribution of catalytic nanoparticles.

In general, single catalyst particle characterization is a powerful tool to understand the intra and inter-particle heterogeneity. However, this same heterogeneity complicates the experimental approach to study the catalytic phenomena. The source of the heterogeneity is in both the synthesis and the catalysis processes while our interests should be focused just on the second by controlling the first. Therefore, we believe that the development of new technologies to synthesise catalyst particles with homogeneous properties can facilitate their study and thus, increase the understanding of the heterogeneity caused during catalysis. For this, we believe that microfluidics can have an important role in the future. The microreactors community has already shown the possibility to fabricate nano- and microparticles with homogeneous properties in regard to: size, composition and porosity, and at high throughput. 
Appendix 


\section{A Appendix Chapter 3}

\section{A.1 Background on the theory of superparamagnetism}

The conventional model for superparamagnetism ${ }^{1}$ was used to estimate the volume of the magnetic clusters from the magnetic hysteresis loops. We assume the particles to be composed of a number of magnetic clusters with identical volume $V_{\text {cluster }}$ and moment $\mu\left(\mathrm{Am}^{2}\right)$. The magnetic moment $m\left(\mathrm{Am}^{2}\right)$ of the particle as a function of the magnetic field $B(\mathrm{~T})$ is fitted to a Langevin curve:

$$
m(B)=m_{0} \mathcal{L}\left(\frac{\mu B}{k T}\right)
$$

where $m_{0}$ is the moment of the particle at saturation (equal to the number of magnetic clusters times their moment $\mu), k_{B}$ is Boltzmann's constant $\left(1.3810^{-23}\right.$ $\mathrm{J} / \mathrm{K}$ ) and $T$ the measurement temperature $(\mathrm{K})$. The susceptibility of the particle, $\chi_{\mathcal{L}}=\partial m / \partial B$, is approximately equal to:

$$
\chi_{\mathcal{L}}=m_{0} \mu B\left(3 k_{B} T\right)^{-1} \text {, }
$$

for temperatures above the blocking temperature. From the Langevin fit, one can estimate the moment of the clusters. Assuming a saturation magnetization for magnetite $\left(M_{\mathrm{s}}=480 \mathrm{kA} / \mathrm{m}\right)$, we can estimate the cluster volume:

$$
V_{\text {cluster }}=\frac{\mu}{M_{\mathrm{s}}}
$$

We define the temperature at which the cluster loses their magnetization within the time of measurement $\left(\tau_{\mathrm{m}}\right)$, the blocking temperature $T_{\mathrm{B}}$ :

$$
\tau_{\mathrm{m}}=\tau_{0} \exp \left(\frac{K V_{\text {cluster }}}{k_{B} T_{\mathrm{B}}}\right)
$$

where $\tau_{0}$ is the attempt time for magnetisation reversal in the cluster and $K$ is the magnetic anisotropy of the material (at most $20 \mathrm{~kJ} / \mathrm{m}^{3}$ for magnetite). For VSM measurements one usually assumes $\ln \left(\frac{\tau_{\mathrm{m}}}{\tau_{0}}\right)$ to be approximately 25 , so we have a second estimate for the volume of the clusters of

$$
V_{\text {cluster }}=\frac{25 k_{B} T_{\mathrm{B}}}{K}
$$

By combining (3) and (5) we obtain

$$
K=\frac{25 k_{B} T_{\mathrm{B}} M_{S}}{\mu}
$$




\section{A.2 Fluidic system and its holder}

a)

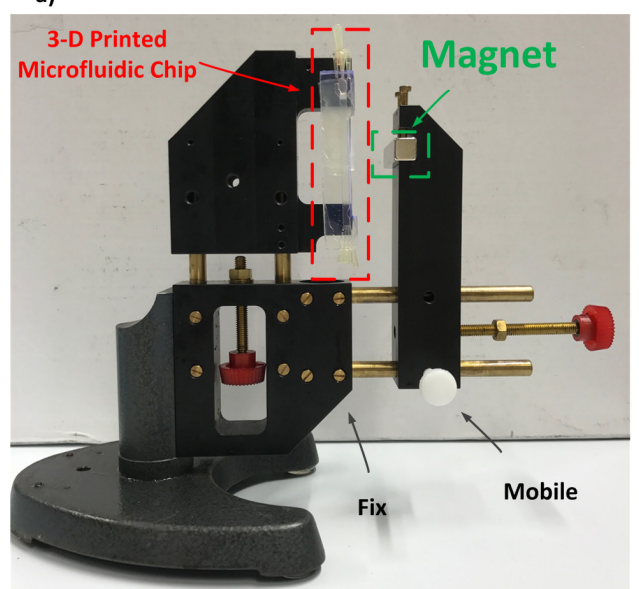

b)

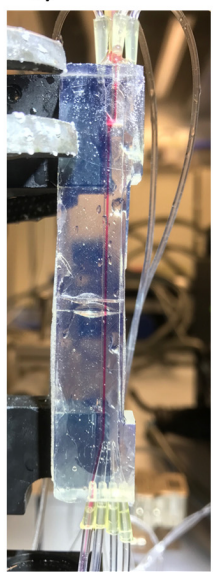

c)

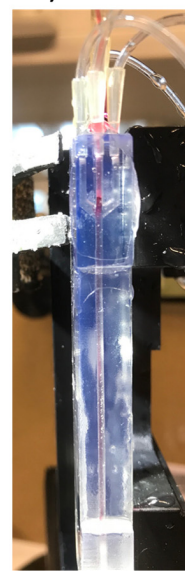

Figure A.1.(a) Chip holder, 3-D microfluidic chip with magnet, (b) front view and (c) side view of the 2-D flow focusing of dye in 3-D microfluidic chip.

Figure A.1a shows the home-made chip holder, which consists of a static part where the 3-D printed chip is placed and a mobile part where the magnet is attached. In Figure A.1b and 1c we can see the front and side view respectively of a red dye introduced in the particle's inlet and 2-D flow focused inside the chip.

\section{A.3 Average size of sorted FCC particles}

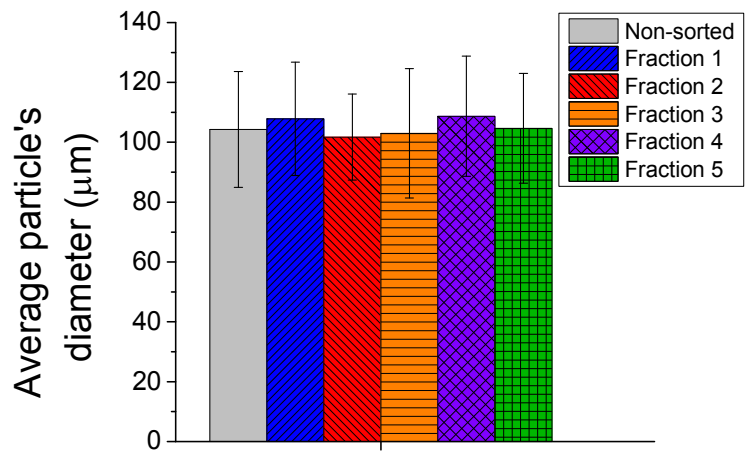

Fractions

Figure A.2. Average particle's diameter of sorted FCC ECAT particles. 


\section{A.4 Magnetic moment of VSM sample holder}

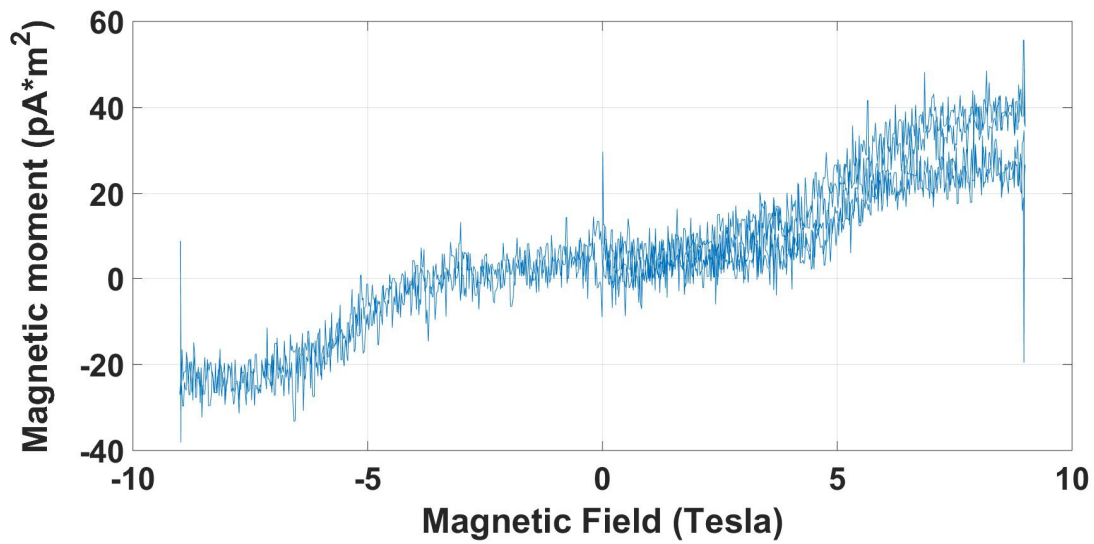

Figure A.3. Magnetic moment of the chip holder with no sample.

Figure A.3 shows a hysteresis loop measurement with no sample showing two steps at -5 and $5 \mathrm{~T}$, which could be related to a paramagnetic behaviour of the device itself. For this reason, magnetic properties were studied in the range of $-4-4 \mathrm{~T}$.

\section{A.5 Micro-XRF average intensities per fraction}

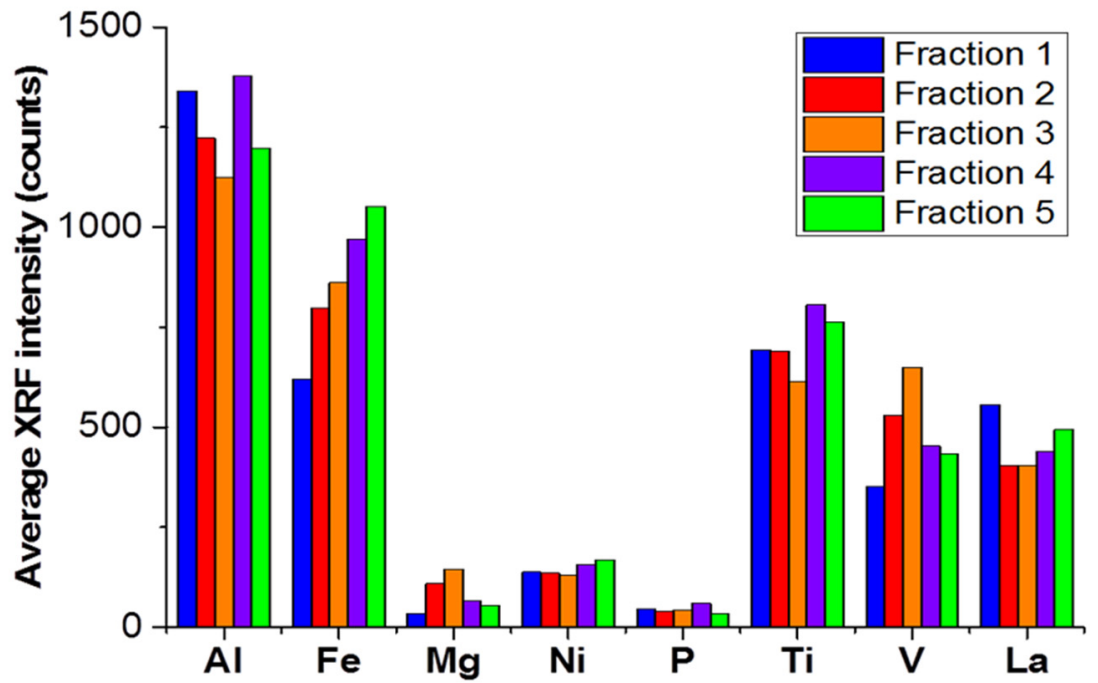

Figure A.4. Micro-XRF average intensities per fraction for $\mathrm{Al}, \mathrm{Fe}, \mathrm{Mg}, \mathrm{Ni}, \mathrm{P}, \mathrm{Ti}, \mathrm{V}$ and $\mathrm{La}$.

Due to the scanning fashion of the micro-XRF measurements, no quantitative data can be obtained from these plots. However, in Figure A.4 it can be seen that the relative intensity for $\mathrm{Mg}$ and $\mathrm{V}$ have the same behaviour 
which confirms the MgO roles as vanadium trap in this FCC ECAT. La, which is very similar in all fractions, it is used as stabilizing ion in zeolite US-Y and it is an indication of the amount of US-Y zeolite domains in the FCC ECAT particles.

\section{A.6 SEM-EDX of black and orange spots on FCC particles}

(a)

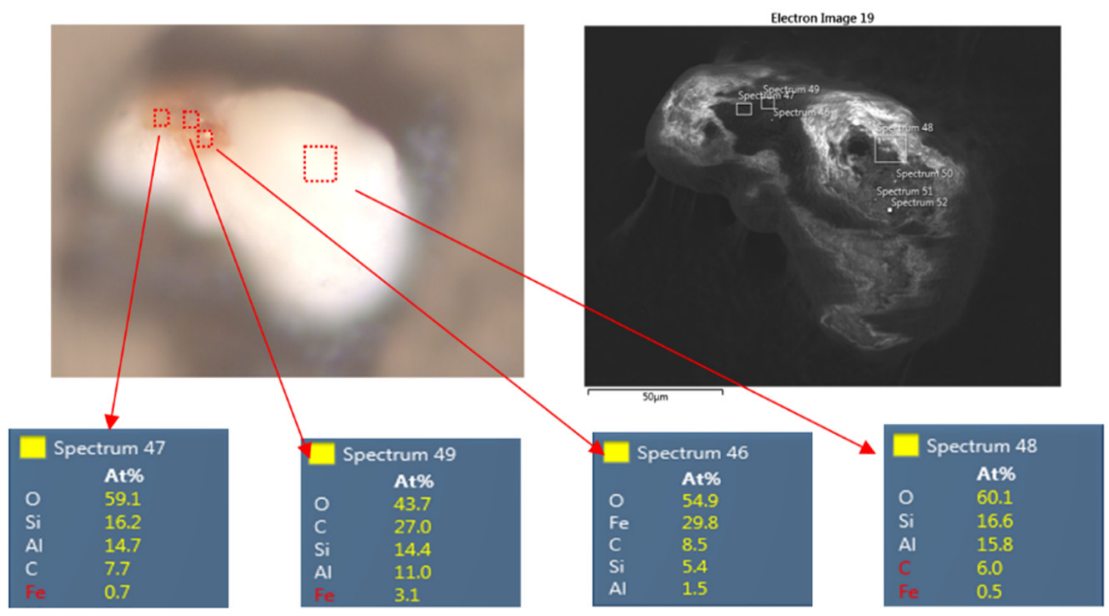

(b)
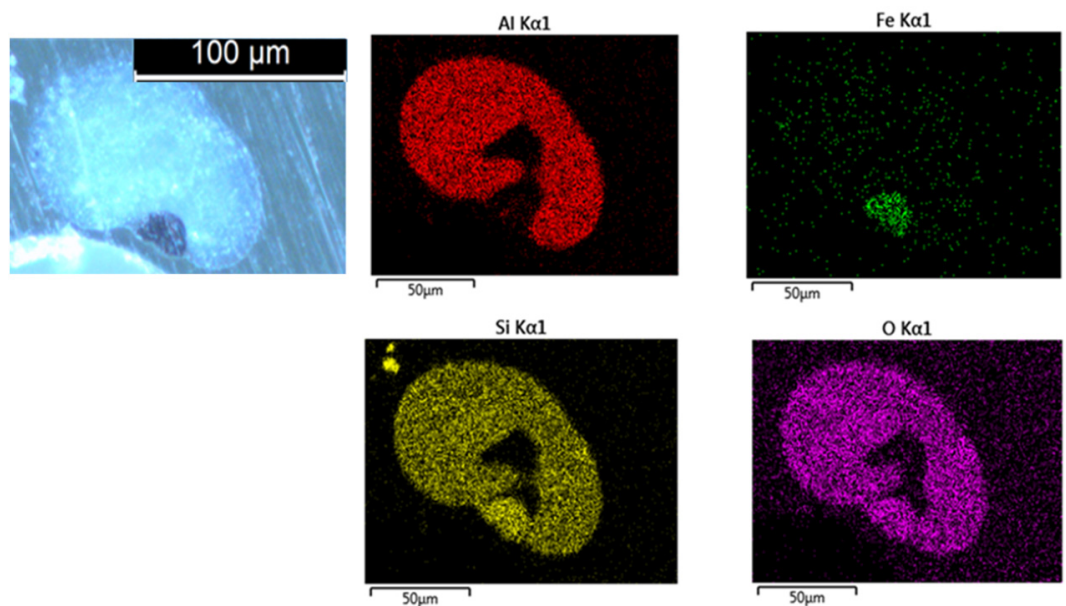

Figure A.5. SEM-EDX measurements showing Al, Si, Fe and $O$ content of (a) of an orange cluster on a sorted FCC ECAT particle and (b) of a black cluster in a sorted FCC ECAT particle embedded in a resin and polished.

Figure A.5a shows a SEM-EDX measurement of a sorted FCC ECAT particle with an orange cluster and it can be seen that the orange cluster is formed by Fe showing two different Fe content levels, spectra 46 and 49. In Figure A.5b it is shown an FCC ECAT particle with a black cluster that was 
embedded in a polymer resin and diced until the black cluster was exposed. EDX elemental mapping shows that the cluster, appearing black on the optical image on the left, shows a high Fe content. At the same time, the $\mathrm{Al}$ concentration at this point is lower while $\mathrm{Si}$ and $\mathrm{O}$ show similar concentrations compared to the rest of the particle. Due to the fact that the black spot couldn't be measured directly from the surface we can conclude that it was deposited under a layer few micrometres thick.

\section{A.7 EPR and UV-vis diffuse reflectance spectra on FCC particles}
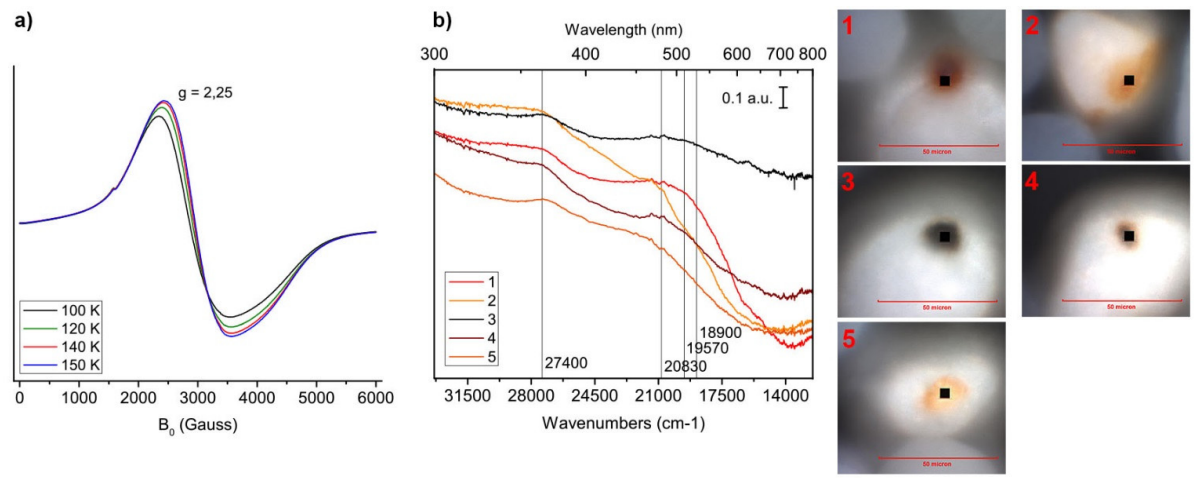

Figure A.6. EPR spectra of fraction F5 at different temperatures show a single broad band with $g$-factor of 2.25 (a) and UV-vis diffuse reflectance spectra of several clusters indicated on the images with 1 to 5 . The band around $27400 \mathrm{~cm}^{-1}$ can be ascribed to $a^{6} A_{1}$ to ${ }^{4} E$ transition, the bands from 20830-18900 $\mathrm{cm}^{-1}$ are from $2\left({ }^{6} \mathrm{~A}_{1}\right)$ to $2\left({ }^{4} \mathrm{~T}_{1}\right)$ transitions and give rise to the color of the iron oxide (b).

The g-value was calculated with the following equation ${ }^{2}$ :

$$
g=\frac{h v}{\mu_{B} B_{0}}
$$

being $h$ the Planck's constant, $v$ the microwave frequency used $[\mathrm{MHz}], \mu_{B}$ the Bohr magneton and $B_{0}$ is the magnetic field peak centre position.

From the EPR data, a broad single band with g-factor of 2.25 is observed, indicating the presence of $\mathrm{Fe}_{\mathrm{x}} \mathrm{O} \mathrm{y}$ in the sample. The absence of a peak with gfactor 3.0 point towards the absence of $\mathrm{Fe}^{\mathrm{II}}$ clusters in our sample. However, EPR is a bulk characterization method and therefore, cannot give information on solely the surface aggregates. Therefore, UV-vis microscopy measurements were performed on several clusters to give more insight in their chemical composition. The spectra in Figure A.6b show features corresponding to both $\alpha-\mathrm{Fe}_{2} \mathrm{O}_{3}$ and $\mathrm{FeO}(\mathrm{OH})$ : both are $\mathrm{Fe}^{\mathrm{III}}$ structures with yellow-orange to red-brown colors. The color of an Fe(III)oxide is due to the position of the EPT band 
(electron pair transition) around 480-530 nm. These ligand field $\mathrm{d}-\mathrm{d}$ transitions are spin forbidden, but have a high intensity due to magnetic coupling of the next to nearest neighbor Fe ${ }^{\mathrm{III}}$. $^{3}$

\section{A.8 Saturation magnetization of sorted FCC particles}
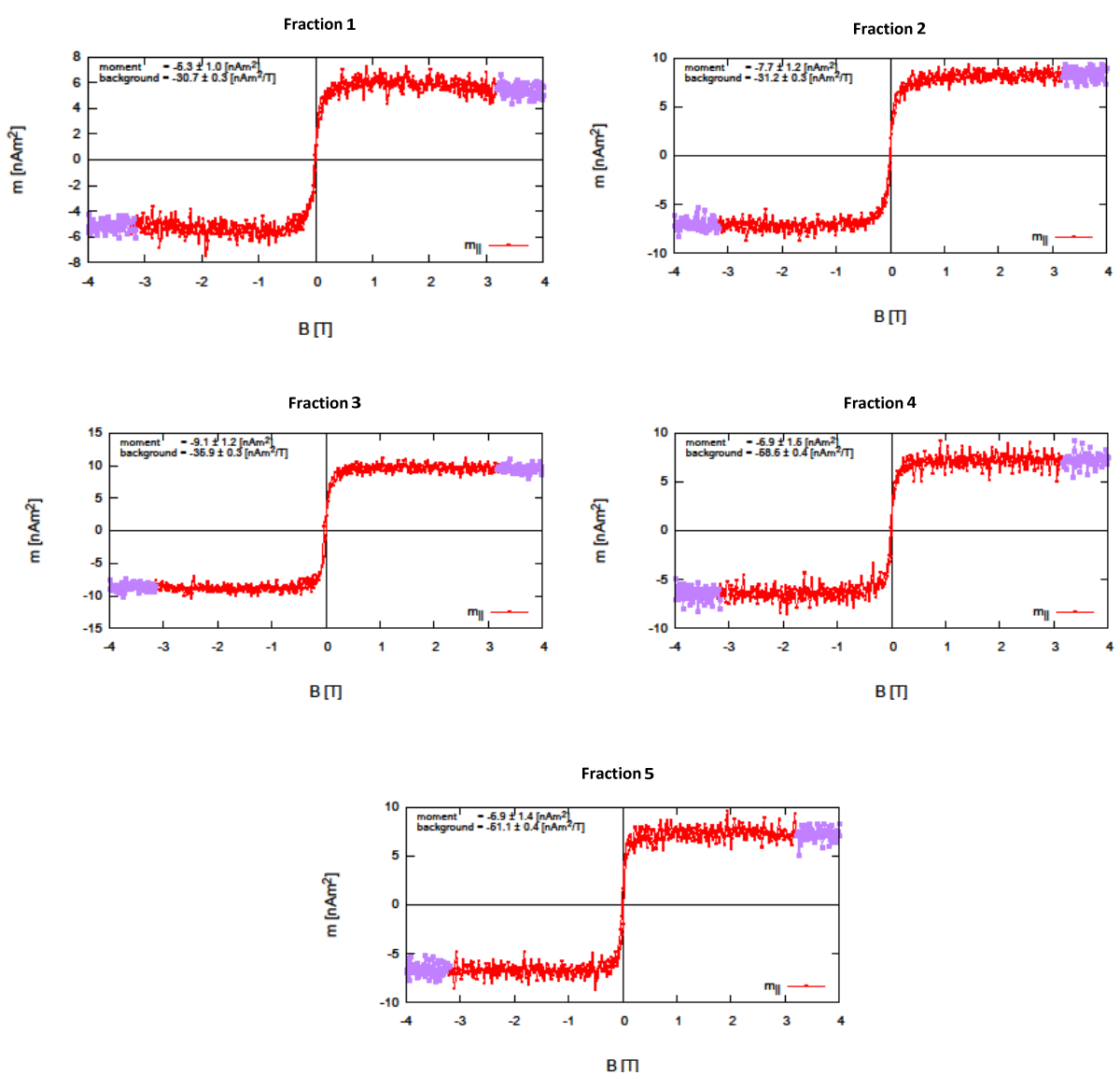

Figure A.7. Experimental magnetic moment and saturation magnetization fit.

The hysteresis of sorted FCC ECAT particles was measured, the diamagnetic background was extracted and the remaining data was fitted with a Langevin function. Figure A.7 shows the moment averaged from the first 10 experimental points at both -4 and $4 \mathrm{~T}$ and the background signal extracted from each fraction. 


\section{A.9 Magnetic moment of sorted FCC particles}
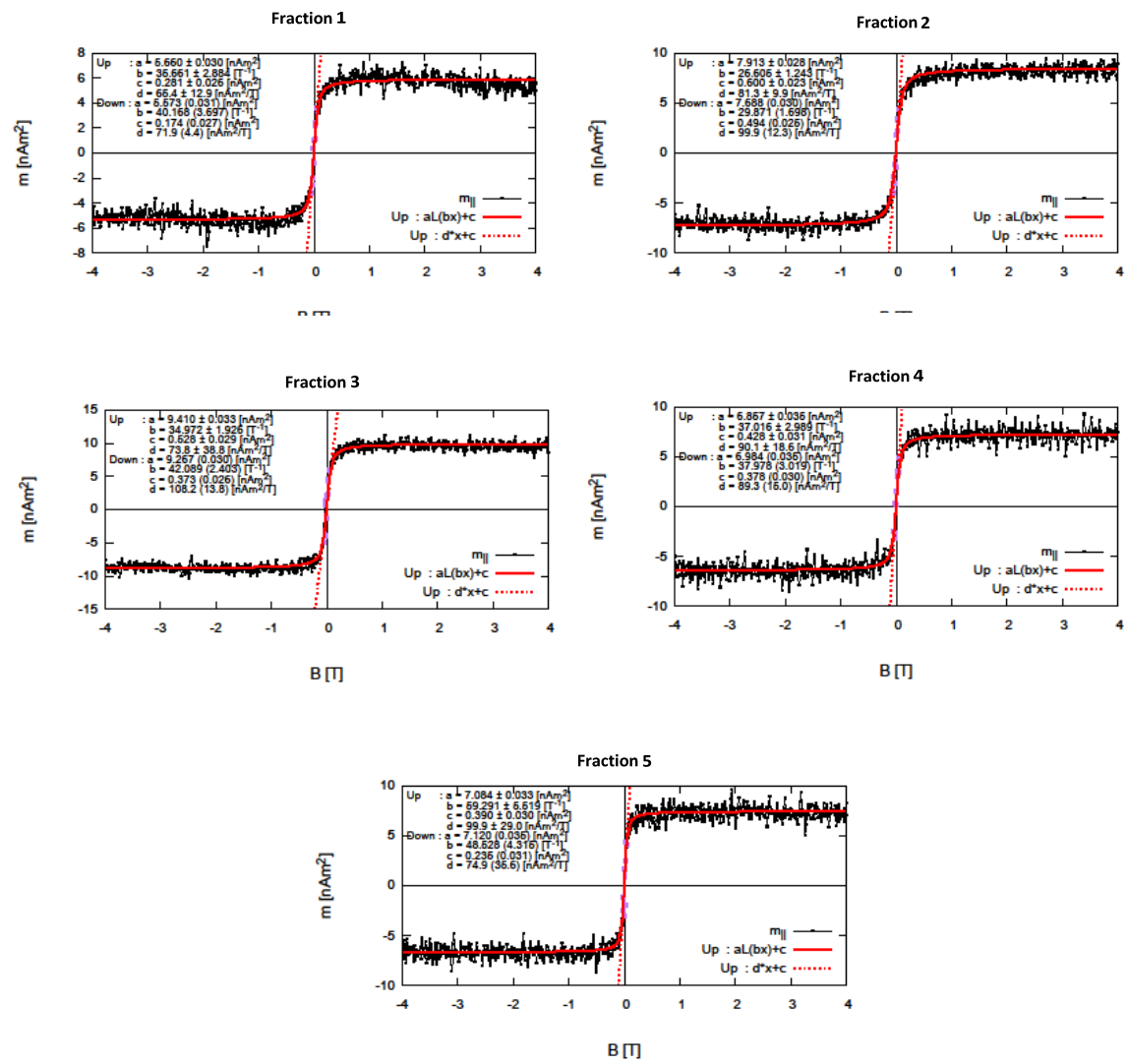

Figure A.8. Experimental magnetic moment Langevin fit and parameters for the different fractions.

Figure A.8 shows the Langevin fitting being, $a$ the saturation magnetization $\left(m_{0}\right)$ and $b$ the Langevin parameter $\left(\mu\left(K_{B} T\right)^{-1}\right)$. Besides, in order to know the susceptibility, the 10 first experimental points in both directions, negative and positive, at low magnetic fields were fitted by a linear equation were $\mathrm{c}$ and $\mathrm{d}$ are their $1^{\text {st }}$ and $2^{\text {nd }}$ coefficients. 


\section{A.10 Fluorescence microscopy of sorted FCC particles}

a)
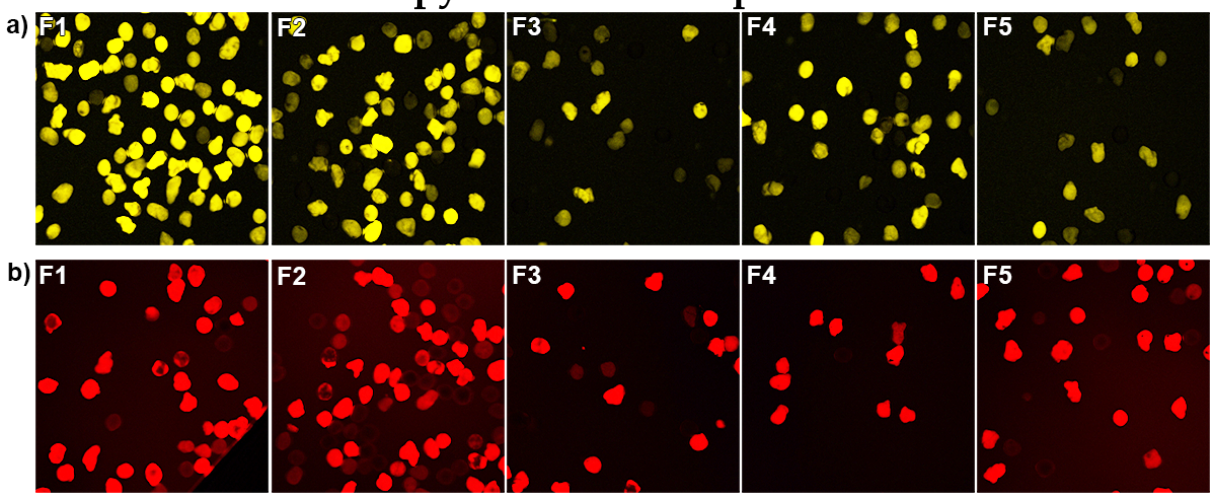

Figure A.9. The fluorescence microscopy images that were used for determining the average fluorescence intensity per particle for 4-methoxystyrene (a) and Nile Blue A (b) staining.

For obtaining the histograms in Figure 3.5 with acidity and accessibility measurements per fraction, images with spectral information of multiple FCC ECAT particles were analysed by determining an average fluorescence per particle using ROI imaging in the Confocal Nikon A1 analysis software. Figure A.9 shows one of these images per fraction. The brightness and contrast are adjusted to visualize the non-fluorescent images.

\section{A.11 References}

1. Bean, C. P. \& Livingston, J. D. Superparamagnetism. J. Appl. Phys. 30, S120-S129 (1959).

2. Hagen, W. R. EPR spectroscopy as a probe of metal centres in biological systems. Dalt. Trans. 4415-4434 (2006).

3. Cornell, R. M. \& Schwertmann, U. Characterization. in The Iron Oxides: Structure, Properties, Reactions, Occurences and Uses 139-183 (Wiley-VHC Verlag GmbH \& Co. KGaA, 2004). 


\section{B Appendix Chapter 4}

\section{B.1 Experimental setup}

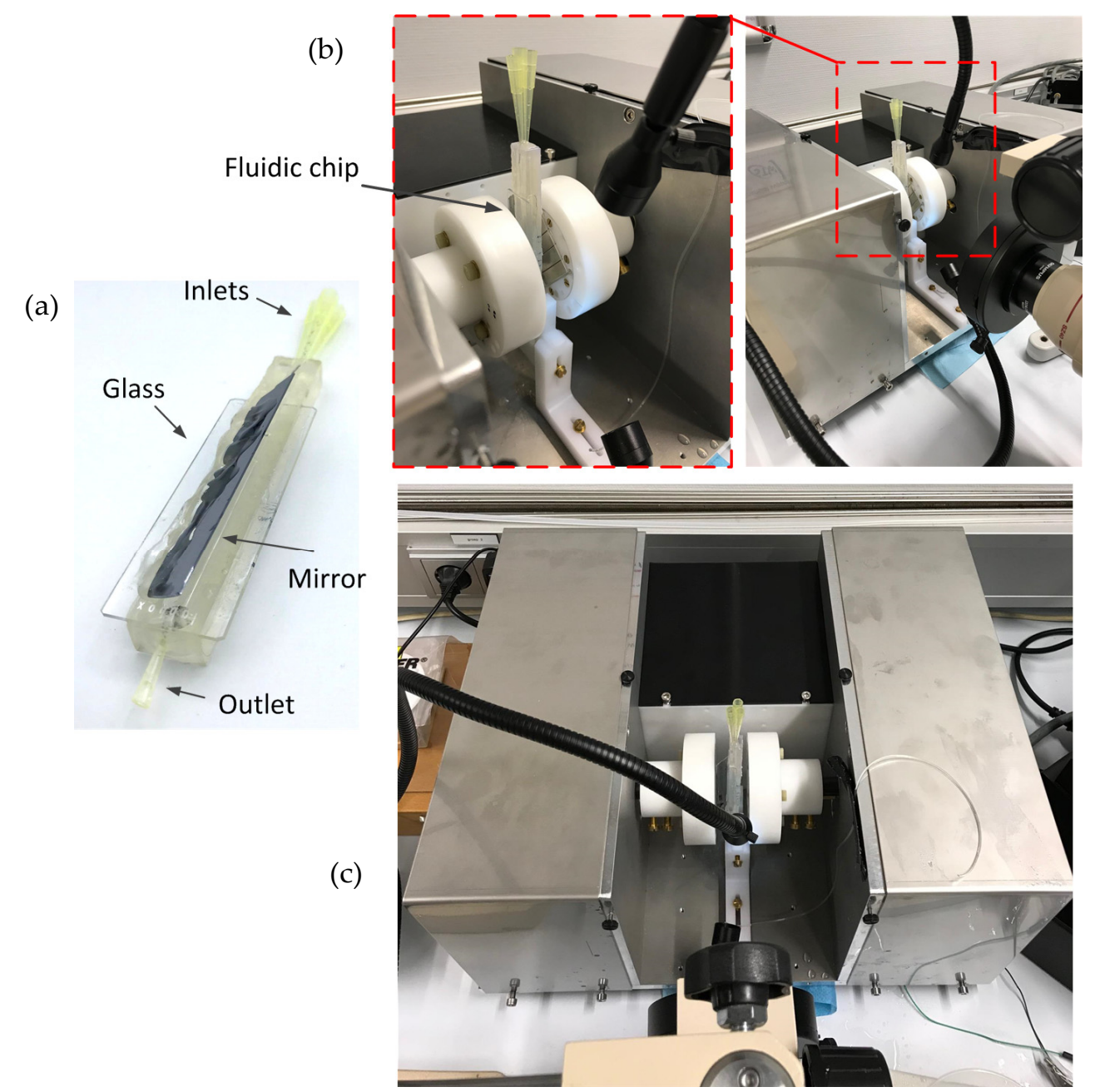

Figure B.1. (a) Image of the fluidic system with a glass to seal the chip and observe the janus particles, a mirror to reflect the trajectories of the janus particles and 3 pipette tips to introduce the fluid and particles. (b) Images of the setup where both magnets are placed at both sides of the fluidic chip. (c) Top-view of the setup. 


\section{B.2 Magnetic moment of the janus particle}

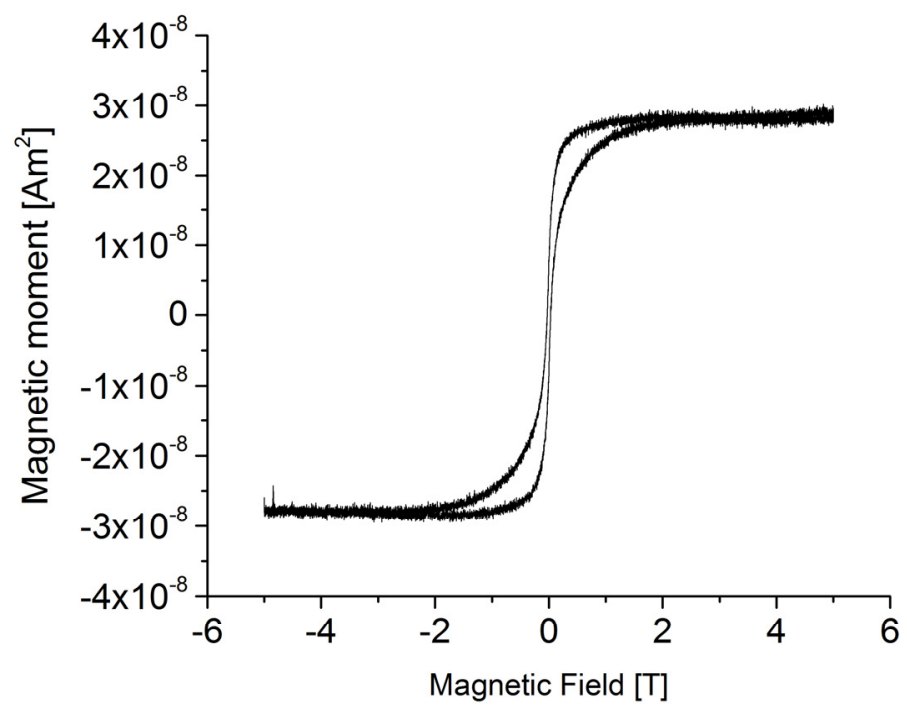

Figure B.2. Magnetic moment of 453 janus particles from -5 to $5 \mathrm{~T}$.

\section{B.3 Zig-zag particles inlet of the fluidic system}

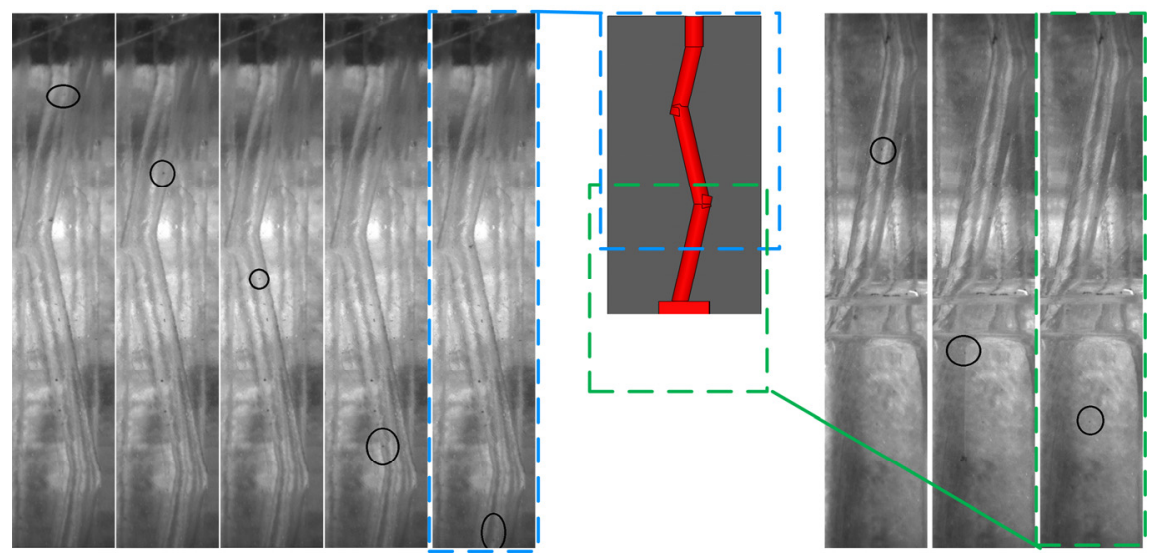

Figure B.3. Zig-zag inlet fabricated to introduce the janus particles at similar starting positions. Inside the black circle a particle rolling down can be followed until entering in the main channel. 


\section{Appendix Chapter 5}

\section{C.1 Design of the microfluidic chip}

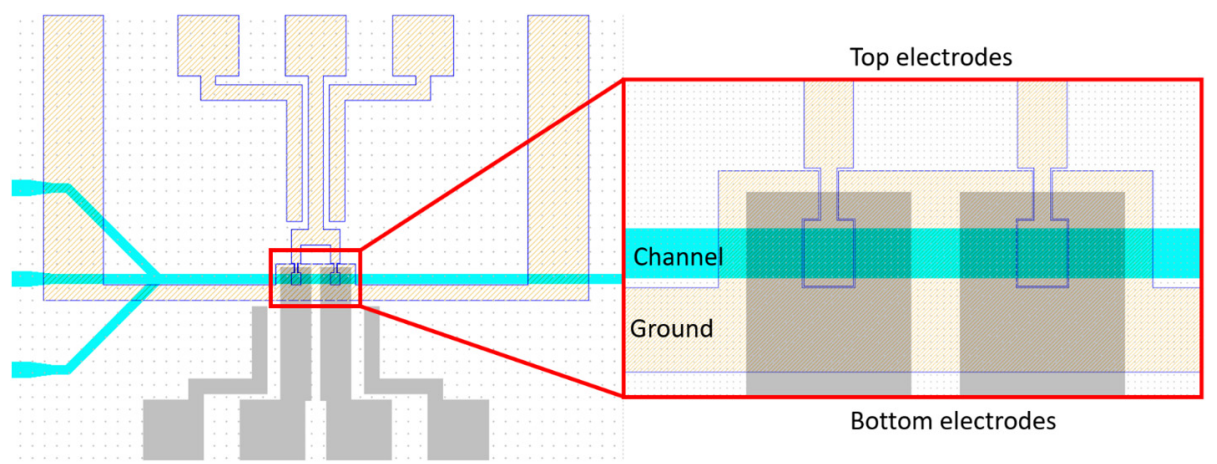

Figure C.1. Combined image of the masks used in the fabrication of the microfluidic chip. In cyan, grey and yellow the mask of the channel, bottom electrodes and top electrodes are shown respectively.

\section{C.2 Experimental setup}

(a)

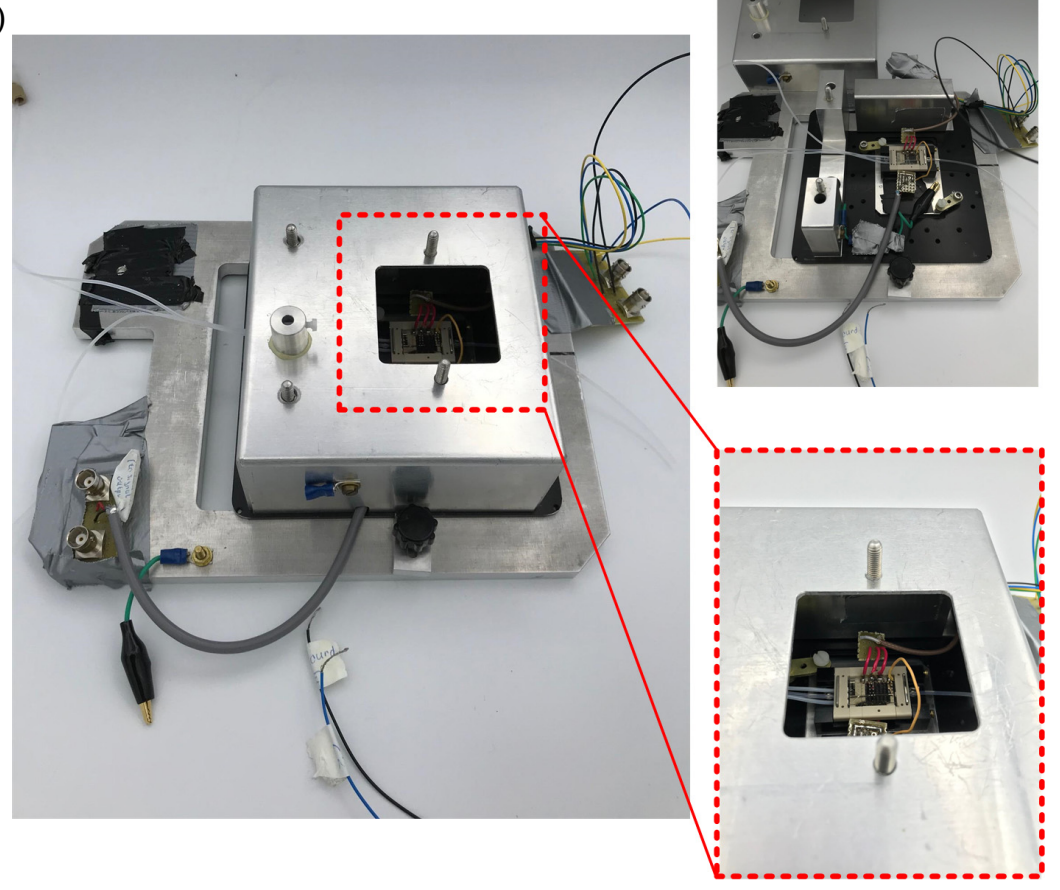

(b)

Figure C.2. Image of the (a) outside and (b) inside of the faraday cage used to measure the opacity of PS and FCC particles. 


\section{C.3 Simulated bode plot}

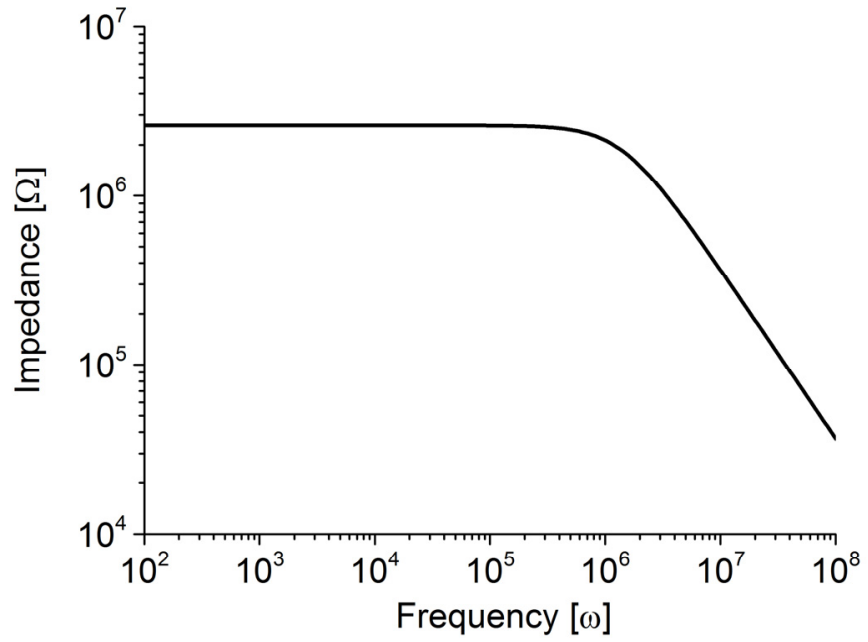

Figure C.3. Simulated bode plot with a $1 \mathrm{mM}$ PBS solution and the cell constant used in the microfluidic chip..

\section{C.4 Masks}

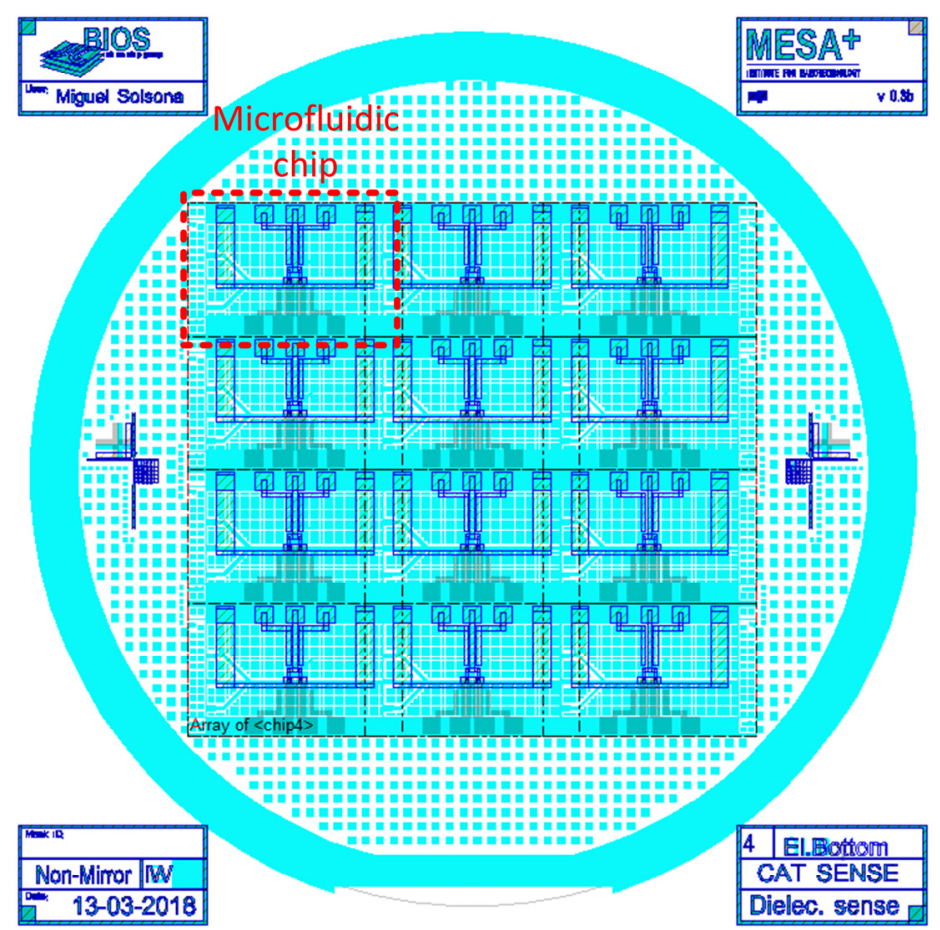

Figure C.4. Combined layout of the three masks that are used. Showing in cyan the SU-8 channel layer, in grey the bottom electrodes and in yellow the top electrodes. 


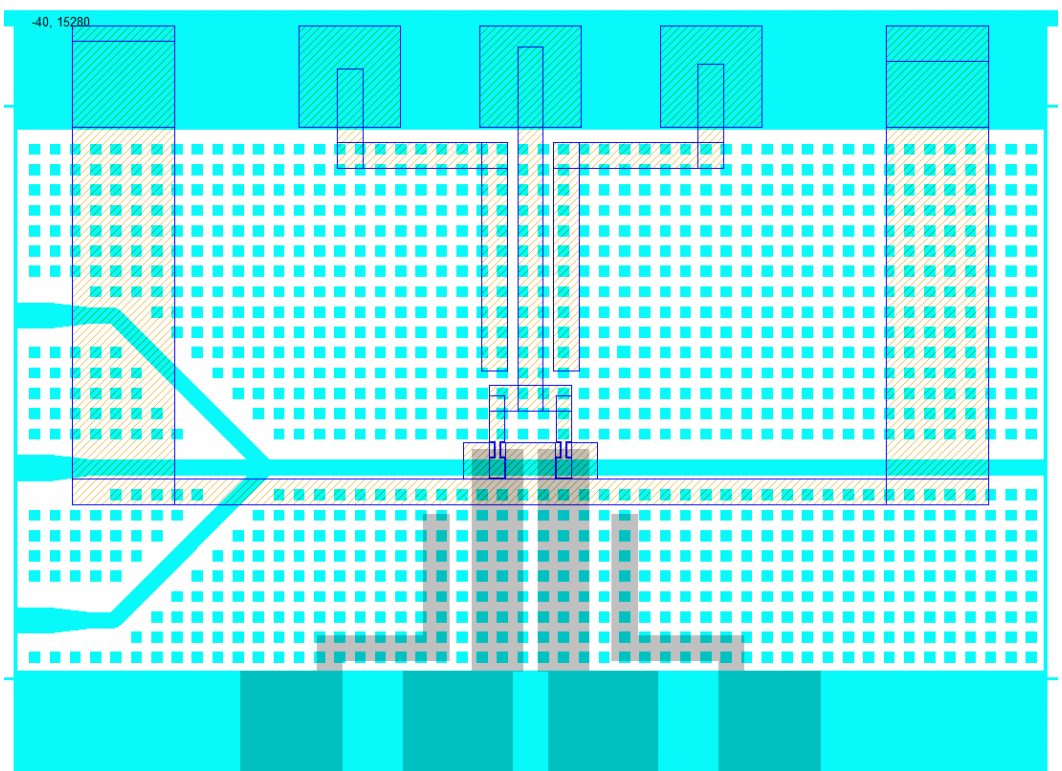

Figure C.5. Combined layout of the three masks that are used in 1 microfluidic chip. Showing in cyan the SU-8 channel layer, in grey the bottom electrodes and in yellow the top electrodes.

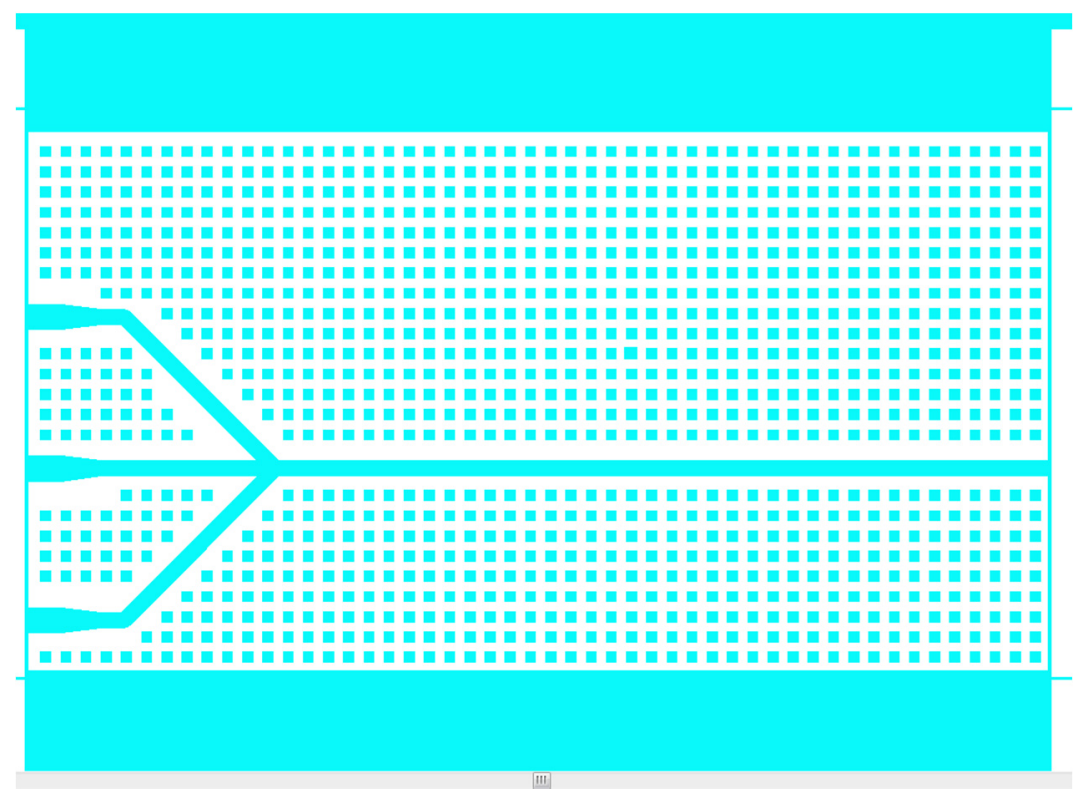

Figure C.6. Mask layout of the SU-8 layer showing the channel structure and including blocks required to avoid wafer deflection due to difference thermal expansion between the SUO layer and the glass wafer. 


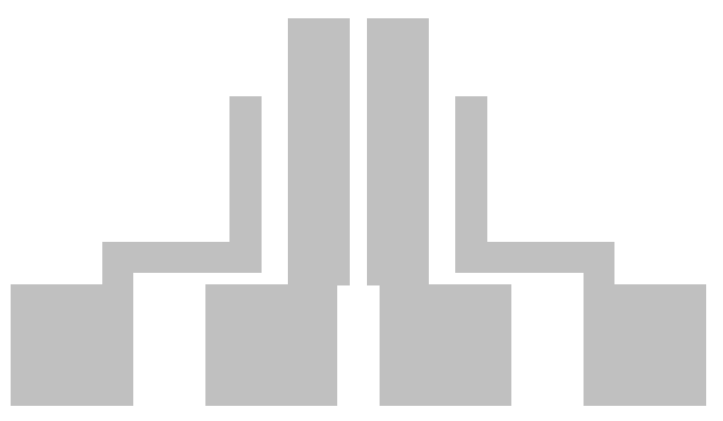

Figure C.7. Mask layout of the bottom wafer electrodes. Showing in the middle the two inputs electrodes which are driven by out-of-phase signals and on both sides the two shielding electrodes.

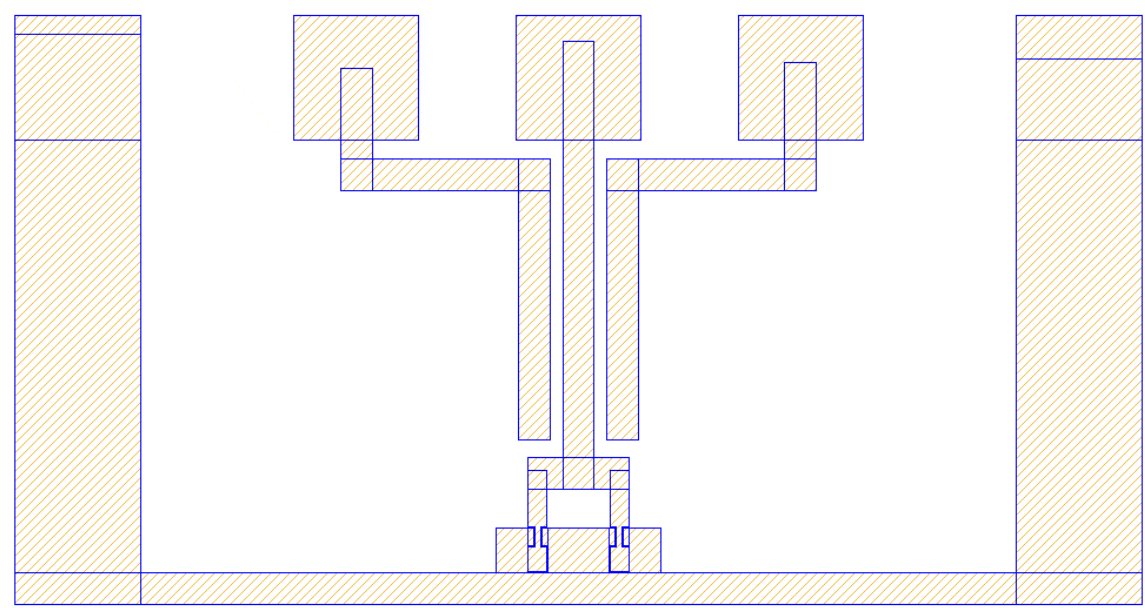

Figure C.8. Mask layout of the top wafer electrodes, showing the two single output electrodes combining in a single electrode. Also, it is shown the shielding electrode around the detection area. 


\section{C.5 Process flow}

\begin{tabular}{|c|c|c|c|}
\hline \multicolumn{4}{|c|}{ Electrodes deposition (Wafers A and B) } \\
\hline & \multicolumn{2}{|c|}{ Process description } & \multirow[t]{2}{*}{ Extra comments } \\
\hline 1 & Select substrate & - $500 \mu \mathrm{m}$ Mempax wafers & \\
\hline 2 & \multirow{9}{*}{$\begin{array}{l}\text { Standard } \\
\text { cleaning } \\
\text { Wet bench } 16\end{array}$} & - $99 \% \mathrm{HNO}_{3}$ (beaker 1, $5 \mathrm{~min}$ ) & \\
\hline 3 & & - $99 \% \mathrm{HNO}_{3}$ (beaker 2, $5 \mathrm{~min}$ ) & \\
\hline 4 & & $\begin{array}{l}\text { - Quick dump rinse (DI resistivity } \\
>10 \Omega \mathrm{M})\end{array}$ & \\
\hline 5 & & - $10 \mathrm{sec} \mathrm{KOH}$ at $75^{\circ}$ & \\
\hline 6 & & - 5 min ultrasound DI water & \\
\hline 7 & & • QDR & \\
\hline 8 & & - 1 min $99 \% \mathrm{HNO}_{3} 2$ times & \\
\hline 9 & & - QDR & \\
\hline 10 & & $\begin{array}{l}\text { - Single wafer spin drying (2500 } \\
\text { rpm, 60s) }\end{array}$ & \\
\hline & \multirow{15}{*}{$\begin{array}{l}\text { Photolithography } \\
\text { Wet bench } 21 \\
\text { EVG620 Mask } \\
\text { Aligner }\end{array}$} & Resist & \\
\hline 11 & & - Dehydration bake $\left(120^{\circ} \mathrm{C}, 5 \mathrm{~min}\right)$ & \\
\hline 12 & & - Spin HDMS (4000 rpm, 30s) & \\
\hline 13 & & - Spin Olin-17 resist (4000rpm, 30s) & \\
\hline 14 & & - Prebake $\left(95^{\circ} \mathrm{C}, 90 \mathrm{~s}\right)$ & \\
\hline & & Exposure & \\
\hline 15 & & $\begin{array}{l}\text { - Exposure: Hg-lamp }\left(12 \mathrm{~mW} / \mathrm{cm}^{2},\right. \\
5 \mathrm{~s})\end{array}$ & \\
\hline 16 & & - Top side, hard-contact & \\
\hline 17 & & Post-exposure bake & \\
\hline 17 & & - Bake $\left(120^{\circ} \mathrm{C}, 60 \mathrm{~s}\right)$ & \\
\hline & & Development & \\
\hline 18 & & $\begin{array}{l}\text { - OPD4262 (beaker } 1 \text { - 30s, beaker } 2 \\
\text { - 15s) }\end{array}$ & \\
\hline 19 & & - Quick dump rinse & \\
\hline 20 & & - Single wafer spin drying & \\
\hline 21 & & - UV-Ozone cleaning (5 min) & \\
\hline 22 & \multirow[t]{3}{*}{$\begin{array}{l}\text { BHF wet etching } \\
\text { Wet bench } 9 / 10\end{array}$} & $\begin{array}{l}\text { - BHF wet etch (etch rate } 22 \\
\mathrm{~nm} / \mathrm{min}, 6 \mathrm{~min} \text { ) }\end{array}$ & \\
\hline 23 & & - Quick dump rinse & \\
\hline 24 & & - Single wafer spin dryer & \\
\hline
\end{tabular}




\begin{tabular}{|c|c|c|}
\hline 25 & $\begin{array}{l}\text { Sputtering Ta } \\
\text { and } \mathrm{Pt} \\
\mathrm{T} \text {-Coathy }\end{array}$ & $\begin{array}{l}\text { - Sputter Ta (deposition rate } 10 \\
\mathrm{~nm} / \mathrm{min} \text { ) } \\
\text { Deposition time for } 10 \mathrm{~nm}=\text { after } 1 \\
\text { min pre-sputter. }\end{array}$ \\
\hline 26 & & $\begin{array}{l}\text { - Sputter Pt (deposition rate } 22 \\
\mathrm{~nm} / \mathrm{min}, 330 \mathrm{sec} \text { ) } \\
\text { Deposition time for } 125 \mathrm{~nm}=300 \mathrm{~s} \\
\text { after } 1 \text { min pre-sputter. }\end{array}$ \\
\hline 27 & $\begin{array}{l}\text { Lift-off } \\
\text { Wet bench } 11\end{array}$ & $\begin{array}{l}\text { - Lift-off in acetone and ultrasound } \\
(1 \mathrm{~min}) \\
\text { - Just acetone ( } 2 \mathrm{~h}) \\
\text { - Acetone and ultrasound ( } 5 \mathrm{~min})\end{array}$ \\
\hline 28 & & - Spray with VLSI isopropanol \\
\hline 29 & & - Single wafer spin drying \\
\hline
\end{tabular}

\begin{tabular}{|c|c|c|c|}
\hline \multicolumn{4}{|c|}{ SU-8 Deposition (Wafer A) } \\
\hline & \multicolumn{2}{|l|}{ Process description } & Extra comments \\
\hline 30 & Select substrate & - Wafer A & \\
\hline 31 & \multirow{4}{*}{$\begin{array}{l}\text { Standard } \\
\text { cleaning } \\
\text { Wet bench } 16\end{array}$} & - $99 \% \mathrm{HNO}_{3}$ (beaker 1, 5 min) & \\
\hline 32 & & - $99 \% \mathrm{HNO}_{3}$ (beaker 2, 5 min) & \\
\hline 33 & & $\begin{array}{l}\text { - Quick dump rinse (DI resistivity } \\
>10.5 \Omega \mathrm{M})\end{array}$ & \\
\hline 34 & & $\begin{array}{l}\text { - Single wafer spin drying ( } 2500 \\
\text { rpm, 60s) }\end{array}$ & \\
\hline 35 & \multirow[t]{4}{*}{$\begin{array}{l}\text { Photolithography } \\
\text { Wet bench } 24\end{array}$} & $\begin{array}{l}\text { - First layer }(200 \mu \mathrm{m}) \\
\text { Spin SU-8 } 100 \text { program } 10 \text { (30s at } \\
2000 \mathrm{rpm})\end{array}$ & \multirow[t]{4}{*}{$\begin{array}{l}\text { Do this same } \\
\text { day as bonding } \\
\text { both wafers }\end{array}$} \\
\hline 36 & & $\begin{array}{l}\text { - Prebake } \\
\text { - } 5 \text { min } 50 \text { degrees } \\
\text { - } 10 \text { min } 65 \text { degrees } \\
\text { - } 120 \text { min } 95 \text { degrees } \\
\text { Cool down to at least } 35 \text { degrees }\end{array}$ & \\
\hline 37 & & $\begin{array}{l}\text { - Second layer }(35 \mu \mathrm{m}) \\
\text { Spin SU-8 } 50 \text { program } 7 \text { (30s at } 4000 \\
\text { rpm) }\end{array}$ & \\
\hline 38 & & $\begin{array}{l}\text { - Prebake } \\
\text { - } 5 \text { min } 50 \text { degrees } \\
\text { - } 5 \text { min } 65 \text { degrees }\end{array}$ & \\
\hline
\end{tabular}




\begin{tabular}{|c|c|c|}
\hline & $\begin{array}{c}\bullet \quad 30 \text { min } 95 \text { degrees } \\
\text { Cool down to at least } 35 \text { degrees }\end{array}$ & \\
\hline \multirow{2}{*}{39} & Exposure & \\
\hline & $\begin{array}{l}\text { - Exposure: Hg-lamp }\left(12 \mathrm{~mW} / \mathrm{cm}^{2} \text {, }\right. \\
55 \mathrm{~s})\end{array}$ & \\
\hline 40 & $\begin{array}{l}\text { - Type of contact (Proximity) } \\
\text { Separation } 10 \mu \mathrm{m}\end{array}$ & \\
\hline \multirow[b]{2}{*}{41} & Post-exposure bake & \\
\hline & $\begin{array}{l}\text { - Bake }\left(80^{\circ} \mathrm{C}, 60 \mathrm{~s}\right) \text { and let the wafer } \\
\text { on the hotplate until it cools down } \\
\text { to } 25^{\circ} \mathrm{C}\end{array}$ & \\
\hline \multirow{2}{*}{42} & Development & \\
\hline & - 25 cycles of 20 seconds & \multirow{2}{*}{$\begin{array}{l}\text { When bonding } \\
\text { both wafers no } \\
\text { hard-bake } \\
\text { needed. }\end{array}$} \\
\hline 43 & $\begin{array}{l}\text { - Quick dump rinse } \\
\text { - Single wafer spin dryer }\end{array}$ & \\
\hline
\end{tabular}

\section{Bonding and dicing (Wafers A and B)}

\begin{tabular}{|c|c|c|c|}
\hline \multicolumn{3}{|c|}{ Process description } & Extra comments \\
\hline 45 & $\begin{array}{l}\text { Select } \\
\text { substrates }\end{array}$ & $\begin{array}{l}\text { - } 500 \mu \mathrm{m} \text { Mempax bottom wafer } \\
\text { with electrodes and SU-8 layer } \\
\text { (Wafer A) } \\
\text { - } 500 \mu \mathrm{m} \text { Mempax top wafer with } \\
\text { electrodes (Wafer B) }\end{array}$ & \\
\hline 46 & \multirow[t]{3}{*}{$\begin{array}{l}\text { Stack alignment } \\
\text { EVG620 \& } \\
\text { anodic bond } \\
\text { chuck }\end{array}$} & $\begin{array}{l}\text { - Load wafers in the anodic bond } \\
\text { chuck. Load wafer B first, process } \\
\text { side face-down. Load wafer A next, } \\
\text { process side face-up. }\end{array}$ & \\
\hline 47 & & $\begin{array}{l}\text { - Manual anodic bond program, } \\
\text { crosshairs, flag before WEC, align } \\
\text { before WEC and reduce WEC to } \\
\text { almost } 0\end{array}$ & \\
\hline 48 & & - Align using alignment marker. & \\
\hline 49 & $\begin{array}{l}\text { Bonding } \\
\text { EV-501 Anodic } \\
\text { bonder }\end{array}$ & $\begin{array}{l}\text { - Set bonding at } 120^{\circ} \mathrm{C} \text { and } 4000 \mathrm{~N} \\
\text { for } 20 \text { minutes and let cool down }\end{array}$ & \\
\hline
\end{tabular}




\begin{tabular}{|c|l|l|l|}
\hline 50 & $\begin{array}{l}\text { Dicing } \\
\text { Loadpoint } \\
\text { microace 3 }\end{array}$ & $\begin{array}{l}\text { - Laminate the wafer onto the } \\
\text { transparent dicing foil by rolling } \\
\text { out a piece of foil over the wafer }\end{array}$ & $\begin{array}{l}\text { - Use the TC300 blade }(300 \mu \mathrm{m} \\
\text { thick) }\end{array}$ \\
\hline
\end{tabular}




\section{Appendix Chapter 6}

\section{D.1 Microfluidic setup}

(b)

(a)
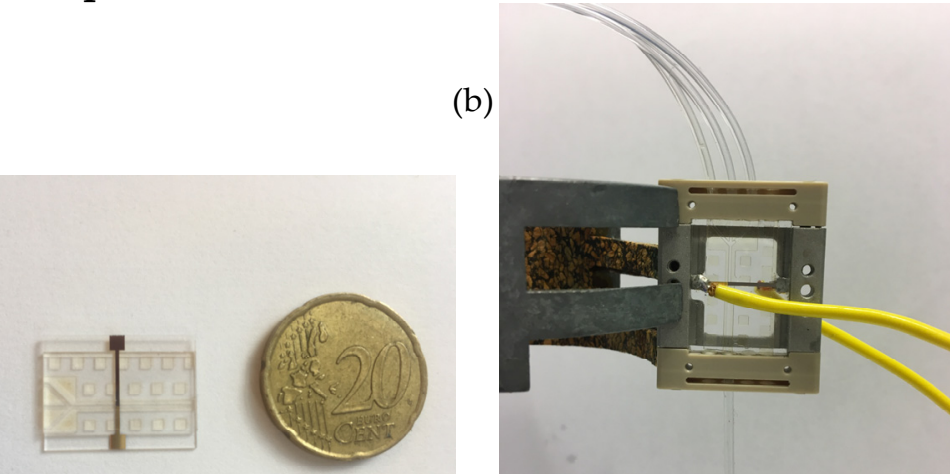

Figure D.1. (a) Microfluidic chip, (b) chip-holder and electrical connections used to detect the position of polystyrene particles $80 \mu \mathrm{m}$ in diameter.

\section{D.2 Cyclic voltammogram}

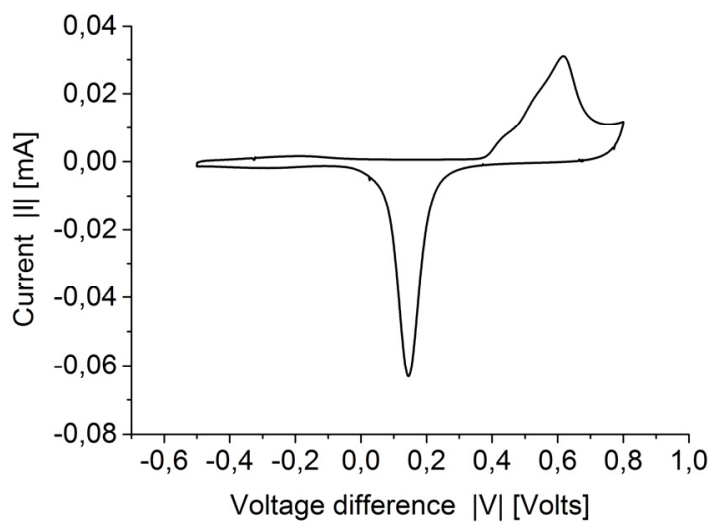

Figure D.2. Cyclic voltammogram of the gold electrodes with exposed regions to be electrodesposited with platinum black in $0.1 \mathrm{M} \mathrm{H}_{2} \mathrm{SO}_{4}$ solution and a scan rate of $10 \mathrm{mV} / \mathrm{s}$ 


\section{D.3 Channel dimensions}

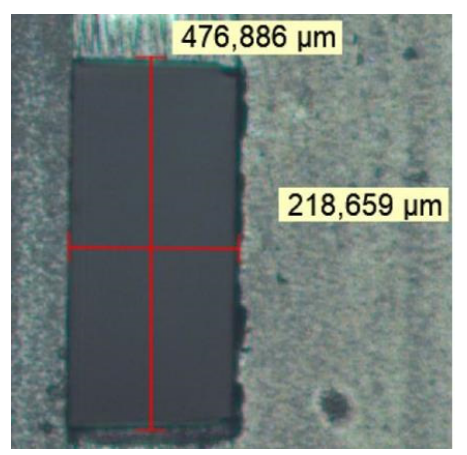

Figure D.3. Experimentally measured dimensions of the microfluidic channel.

\section{D.4 Particle size histogram}

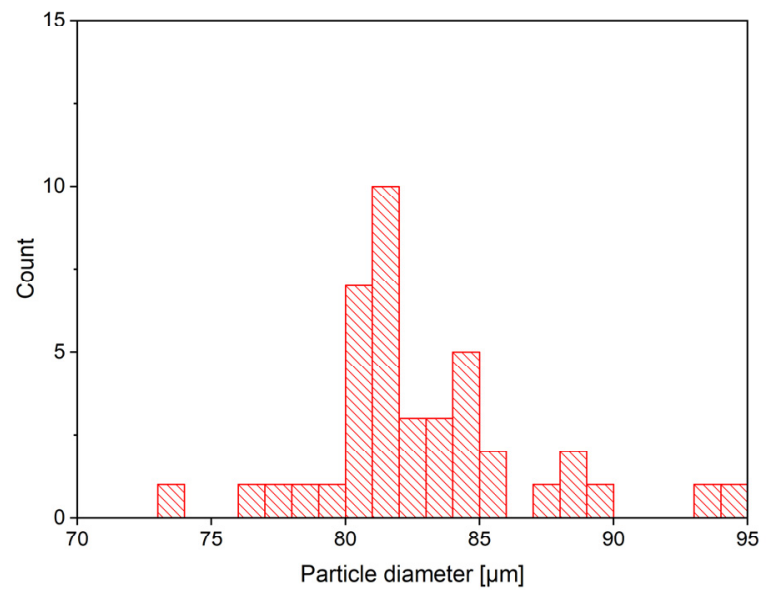

Figure D.4. Particle size distribution of 41 polystyrene particles. 


\section{D.5 Simulation results}

Table D.1. Simulated impedance of polystyrene particles with different radius at high frequency.

\begin{tabular}{|c|c|c|c|}
\hline Radius $(\boldsymbol{\mu m})$ & $\begin{array}{c}\text { Frequency } \\
(\mathbf{K H z})\end{array}$ & $\begin{array}{c}\text { Impedance } \\
(\boldsymbol{\Omega})\end{array}$ & $\begin{array}{c}\text { Impedance difference } \\
\text { with a } \mathbf{3 7} \boldsymbol{\mu \mathbf { m }} \mathbf{( \Omega )}\end{array}$ \\
\hline 37 & 100 & 721,13 & 0,00 \\
\hline 39 & 100 & 721,75 & 0,61 \\
\hline 41,5 & 100 & 722,60 & 1,47 \\
\hline 44 & 100 & 723,56 & 2,43 \\
\hline 47 & 100 & 724,88 & 3,74 \\
\hline
\end{tabular}

$722.54 \Omega$

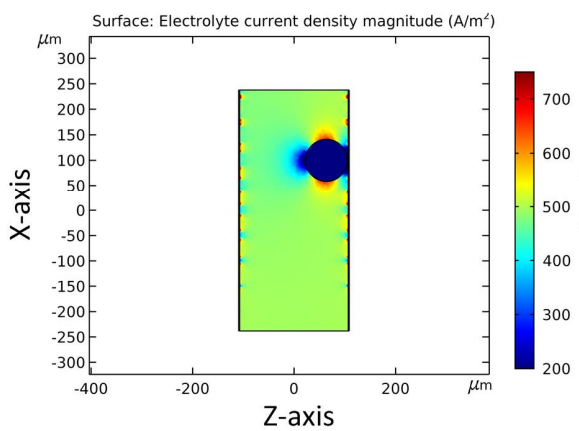

$722.23 \Omega$

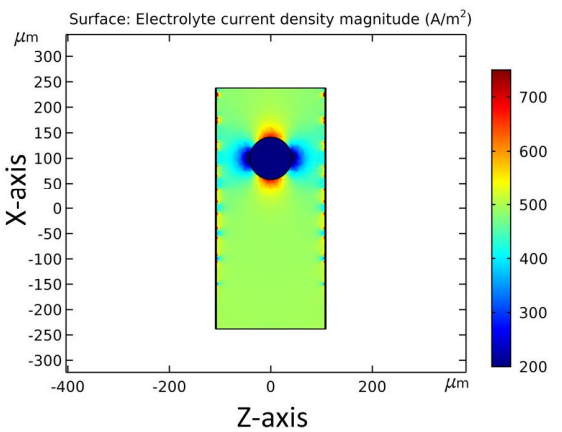

Figure D.5.Simulated impedance of polystyrene particles at two z-axis positions and at high frequency.

\section{D.6 Average distance to the mean}

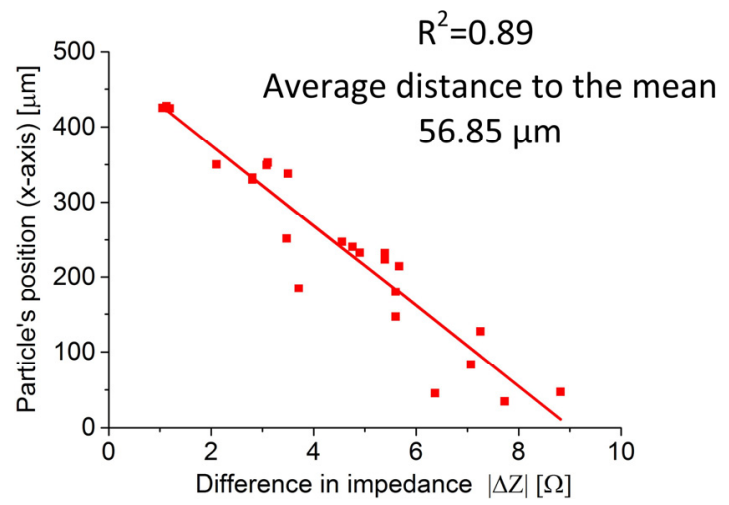

Figure D.6. Linear trendline of the experimental data at $800 \mathrm{~Hz}$ with $R^{2}$ and average distance of the experimental data to the trendline. 


\section{E Appendix Chapter 8}

\section{E.1 Masks}

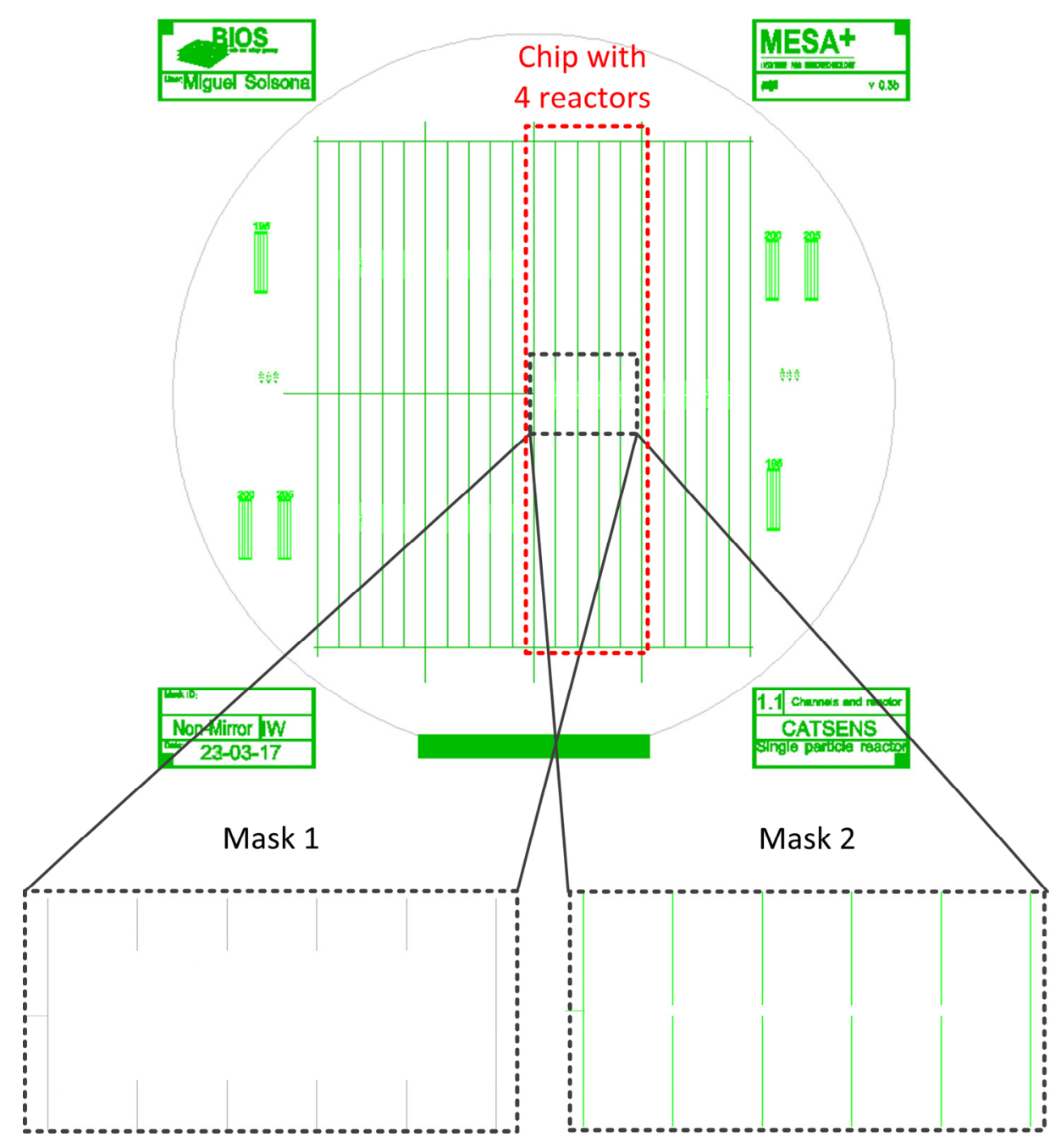

Figure E.1. The two masks used to fabricate the single particle reactor chip. Each of the masks were used to etch a different wafer. 


\section{E.2 Process flow}

\begin{tabular}{|c|c|c|c|}
\hline \multicolumn{4}{|c|}{ Channel etch (Wafers A and B) } \\
\hline & \multicolumn{2}{|c|}{ Process description } & Extra comments \\
\hline 1 & Select substrate & - $500 \mu \mathrm{m}$ Mempax top wafer & \\
\hline 2 & \multirow{9}{*}{$\begin{array}{l}\text { Standard } \\
\text { cleaning } \\
\text { Wet bench } 16\end{array}$} & - $99 \% \mathrm{HNO}_{3}$ (beaker 1, $5 \mathrm{~min}$ ) & \\
\hline 3 & & - $99 \% \mathrm{HNO}_{3}$ (beaker 2, $5 \mathrm{~min}$ ) & \\
\hline 4 & & $\begin{array}{l}\text { - Quick dump rinse (DI resistivity } \\
>10 \Omega \mathrm{M} \text { ) }\end{array}$ & \\
\hline 5 & & - $10 \mathrm{sec} \mathrm{KOH}$ at $75^{\circ}$ & \\
\hline 6 & & - 5 min ultrasound DI water & \\
\hline 7 & & • QDR & \\
\hline 8 & & - $1 \mathrm{~min} 99 \% \mathrm{HNO}_{3} 2$ times & \\
\hline 9 & & • QDR & \\
\hline 10 & & $\begin{array}{l}\text { - Single wafer spin drying (2500 } \\
\text { rpm, 60s) }\end{array}$ & \\
\hline 11 & \multirow[t]{2}{*}{$\begin{array}{l}\text { Sputtering } \mathrm{Cr} \\
\text { and } \mathrm{Au} \\
\text { T-Coathy }\end{array}$} & $\begin{array}{l}\text { - Sputter Cr (deposition rate } 15 \\
\mathrm{~nm} / \mathrm{min} \text { ) } \\
\text { Deposition time for } 30 \mathrm{~nm}=2 \mathrm{~min} \\
\text { after } 1 \mathrm{~min} \text { pre-sputter. }\end{array}$ & \\
\hline 12 & & $\begin{array}{l}\text { - Sputter Au (deposition rate } 50 \\
\mathrm{~nm} / \mathrm{min} \text { ) } \\
\text { Deposition time for } 150 \mathrm{~nm}=3 \mathrm{~min} \\
\text { after } 1 \text { min pre-sputter. }\end{array}$ & \\
\hline \multirow{2}{*}{13} & \multirow{11}{*}{$\begin{array}{l}\text { Photolithography } \\
\text { Wet bench } 21 \\
\text { EVG620 Mask } \\
\text { Aligner }\end{array}$} & Resist & \\
\hline & & - Dehydration bake $\left(120^{\circ} \mathrm{C}, 5 \mathrm{~min}\right)$ & \\
\hline \multirow{3}{*}{$\begin{array}{l}14 \\
15 \\
\end{array}$} & & - Spin Olin-17 (4000 rpm, 30s) & \\
\hline & & - Prebake $\left(95^{\circ} \mathrm{C}, 90 \mathrm{~s}\right)$ & \\
\hline & & Exposure & \\
\hline 16 & & $\begin{array}{l}\text { - Exposure: Hg-lamp }\left(12 \mathrm{~mW} / \mathrm{cm}^{2},\right. \\
5 \mathrm{~s})\end{array}$ & \\
\hline 17 & & - Top side, hard-contact & \\
\hline \multirow{2}{*}{18} & & After-exposure bake & \\
\hline & & - Bake $\left(120^{\circ} \mathrm{C}, 60 \mathrm{~s}\right)$ & \\
\hline \multirow[b]{2}{*}{19} & & Development & \\
\hline & & $\begin{array}{l}\text { - OPD4262 (beaker } 1 \text { - 30s, beaker } 2 \\
\text { - 15s) }\end{array}$ & \\
\hline
\end{tabular}




\begin{tabular}{|c|c|c|c|}
\hline 20 & & - Quick dump rinse & \\
\hline 21 & & - Single wafer spin drying & \\
\hline \multirow{2}{*}{22} & & Post-exposure bake & \\
\hline & & - Post-bake $\left(120^{\circ} \mathrm{C}, 10 \mathrm{~min}\right)$ & \\
\hline 23 & \multirow[t]{2}{*}{$\begin{array}{l}\text { Lamination } \\
\text { Dicing room }\end{array}$} & $\begin{array}{l}\text { - Cut blue dicing foil and stick it on } \\
\text { the back side of the wafer }\end{array}$ & \\
\hline 24 & & - UV-Ozone cleaning (5 min) & \\
\hline 25 & \multirow[t]{2}{*}{$\begin{array}{l}\text { Wet etching } \\
\text { Wet bench } 10\end{array}$} & $\begin{array}{l}\text { - Wet etch with gold etchant } \\
\left(\mathrm{KI} / \mathrm{I}_{2}\right) \text {, until Cr color appears. } \approx 1 \\
\text { min. }\end{array}$ & \\
\hline 26 & & $\begin{array}{l}\text { - Wet etch with commercial } \mathrm{Cr} \\
\text { etchant }\left(\mathrm{Ce}\left(\mathrm{NH}_{4}\right)_{2}\left(\mathrm{NO}_{3}\right)_{6}\right. \\
\left.\mathrm{HClO}_{4}\right) \text { Until it becomes } \\
\text { transparent. } \approx 20 \mathrm{~s} \text {. }\end{array}$ & $\begin{array}{l}\text { Don't etch too } \\
\text { long }\end{array}$ \\
\hline 27 & \multirow[t]{3}{*}{$\begin{array}{l}\text { Glass wet etching } \\
\text { Wet bench } 9\end{array}$} & $\begin{array}{l}\text { - Wet etch the glass with }(25 \% \mathrm{HF} \text {, } \\
\left.75 \% \mathrm{H}_{2} \mathrm{O}\right) \text { etch rate } 1 \mu \mathrm{m} / \mathrm{min} \\
200 \mathrm{~min}\end{array}$ & \multirow[t]{2}{*}{$\begin{array}{l}\text { Change position } \\
\text { for temperature } \\
\text { differences }\end{array}$} \\
\hline 28 & & $\begin{array}{l}\text { - Quick dump rinse (DI resistivity } \\
>10 \Omega \mathrm{M})\end{array}$ & \\
\hline 29 & & $\begin{array}{l}\text { - Single wafer spin drying ( } 2500 \\
\text { rpm, 60s) }\end{array}$ & \\
\hline 30 & Delamination & $\begin{array}{l}\text { - Peel off the dicing foil from the } \\
\text { back wafer }\end{array}$ & \\
\hline 31 & \multirow{4}{*}{$\begin{array}{l}\text { Standard } \\
\text { cleaning } \\
\text { Wet bench } 16\end{array}$} & - $99 \% \mathrm{HNO}_{3}$ (beaker 1, $5 \mathrm{~min}$ ) & \\
\hline 32 & & - $99 \% \mathrm{HNO}_{3}$ (beaker 2, $5 \mathrm{~min}$ ) & \\
\hline 33 & & $\begin{array}{l}\text { - Quick dump rinse (DI resistivity } \\
>10.5 \Omega \mathrm{M})\end{array}$ & \\
\hline 34 & & $\begin{array}{l}\text { - Single wafer spin drying (2500 } \\
\text { rpm, 60s) }\end{array}$ & \\
\hline
\end{tabular}

\section{Bonding and dicing (Wafers A and B)}

\begin{tabular}{|l|l|l|l|}
\hline \multicolumn{3}{|l|}{ Process description } & Extra comments \\
\hline 35 & $\begin{array}{l}\text { Select } \\
\text { substrates }\end{array}$ & $\bullet$ Wafer A and B & \\
\hline 36 & Bonding & Clean the wafers & \\
\hline
\end{tabular}




\begin{tabular}{|c|c|c|c|}
\hline 37 & & $\begin{array}{l}\text { - } 15 \text { min Piranha }\left(\mathrm{H}_{2} \mathrm{SO}_{4}: \mathrm{H}_{2} \mathrm{O}_{2} 3: 1\right. \\
\mathrm{v}: \mathrm{v})\end{array}$ & \\
\hline 38 & & - $99 \% \mathrm{HNO}_{3}$ (beaker 2, 5 min) & \\
\hline 39 & & $\begin{array}{l}\text { - Quick dump rinse (DI resistivity } \\
>10.5 \Omega \mathrm{M})\end{array}$ & \\
\hline 40 & & $\begin{array}{l}\text { - Single wafer spin drying }(2500 \\
\text { rpm, 60s })\end{array}$ & \\
\hline 41 & & $\begin{array}{l}\text { - Load wafers in the anodic bond } \\
\text { chuck. Load top wafer B first, } \\
\text { process side face-down. Load } \\
\text { bottom wafer A next, process side } \\
\text { face-up. }\end{array}$ & \\
\hline 42 & & $\begin{array}{l}\text { - Manual anodic bond program, } \\
\text { crosshairs, flag before WEC, align } \\
\text { before WEC and reduce WEC to } \\
\text { almost } 0\end{array}$ & \\
\hline 43 & & - Align using alignment markers. & \\
\hline 44 & & Pre-bond the wafers & \\
\hline 45 & & $\begin{array}{l}\text { - Put together and if necessary } \\
\text { wipe out any air inclusions }\end{array}$ & \\
\hline 46 & & $\begin{array}{l}\text { Anneal in convection furnace (in } \\
\text { air) }\end{array}$ & \\
\hline 47 & & - Ramp up to $625^{\circ} \mathrm{C}$ in 5 hours & \\
\hline 48 & & - Keep at $625^{\circ} \mathrm{C}$ for 1 hour & \\
\hline 49 & & - Cool down to RT in 10 hours & \\
\hline 50 & \multirow[t]{2}{*}{$\begin{array}{l}\text { Dicing } \\
\text { Loadpoint micro } \\
\text { ace } 3\end{array}$} & $\begin{array}{l}\text { - Laminate the wafer onto the } \\
\text { transparent dicing foil by rolling } \\
\text { out a piece of foil over the wafer }\end{array}$ & \\
\hline 51 & & $\begin{array}{l}\text { - Use the TC300 blade }(300 \mu \mathrm{m} \\
\text { thick) }\end{array}$ & \\
\hline
\end{tabular}




\section{E.3 Experimental setup}
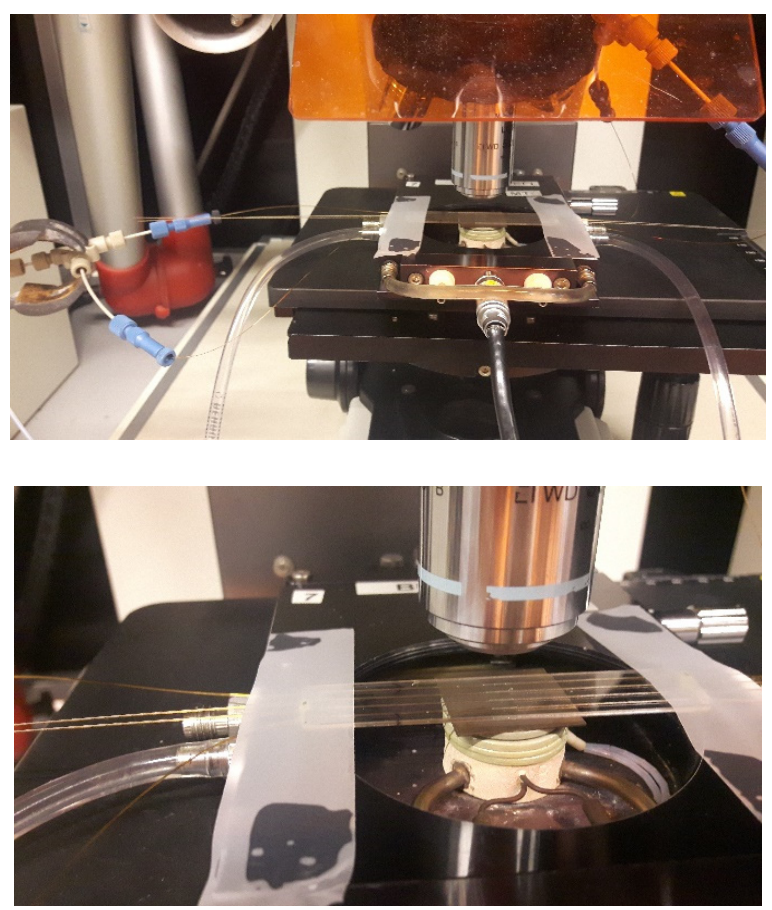

Figure E.2. Overview of the setup containing the micro-reactors, the Linkam cell with the heater, microscope and capillaries. The chip was heated up by the Linkam cell and a silicon wafer piece was placed in between in order to elevate the chip and enhance the heat transfer. 


\section{Scientific Output}

\section{Peer-reviewed journal articles}

Solsona M.*, Nieuwelink A-E.*, Meirer F., Abelmann L., Odijk M., Olthuis W., Weckhuysen B.M., van den Berg A., Magnetophoretic Sorting of Single Catalyst Particles. Angewandte Chemie-International Edition, 57(33), 10589-10594 (2018)

* Both authors contributed equally to this work

Solsona M.*, Westerbeek E.Y.*, Bomer J.C., Olthuis W., van den Berg A. Gradient in Electric Field for Particle Position Detection in Microfluidic Channels. Lab on a chip, 19, 1054-1059 (2019)

* Both authors contributed equally to this work

Solsona M.*, Papadimitriou V.A.*, Olthuis W., van den Berg A., Eijkel, J.C.T., Ion Concentration Polarization for Micro-Particle Mesoporosity Differentiation. Submitted

* Both authors contributed equally to this work

Solsona M.*, Vollenbroek J.C.*, Tregouet C.B.M.*, Nieuwelink A-E., Olthuis W., Weckhuysen B.M., van den Berg A., Odijk M., Microfluidics and Catalyst Particles. Submitted

* Authors contributed equally to this work

Solsona M., Keizer H., Olthuis W., de Boer H.L., Abelmann L., van den Berg A., Force and Deflection on Magnetic Spinning Microparticles. In preparation.

Solsona M., Hendrix J., Odijk M., Olthuis W., van den Berg A. Impedancebased Microfluidic Particle characterization system with Atto-Farad Sensitivity. In preparation.

Mohanty, S., Siciliani de Cumis, H., Solsona, M., Misra, S., Bi-directional Transportation of micro-Agents Induced by Symmetric-Broken Acoustic Streaming AIP Advances, 9 035352-1-035352-3 (2019) 


\section{Conference contributions}

Solsona M., Olthuis W., van den Berg A. Eurosensors XXX, (Poster presentation), Gradient Capacitance for Solid Particle Position Detection in Electrolyte, September 4-7, 2016, Budapest, Hungary.

Solsona M., Odijk M., Olthuis W., van den Berg A. NCCC 2016 (Poster presentation) Impedance Spectroscopy for Sensing the Activity of Catalyst Particles, March 7-9, 2016, Noordwijkerhout, The Netherlands.

Solsona M., Nieuwelink A-E., Vollenbroek J.C., Meirer F., Odijk M., Olthuis W., van den Berg A. Weckhuysen, B.M., NAM 25 (poster presentation) Single Fluid Catalytic Cracking Particle Activity Sorting using Magnetophoresis, June 49, 2017, Denver, United States.

Solsona M., Olthuis W., Vollenbroek J.C., Odijk M., Nieuwelink A-E., Weckhuysen B.M., van den Berg A. NCCC 2017 (Poster presentation) Magnetophoresis for single Fluid Catalytic Cracking particle activity sorting, March 4-6, 2017, Noordwijkerhout, The Netherlands.

Solsona M., Nieuwelink A.E., Papadimitriou V., Eijkel J.C.T., Meirer F., Abelmann L., Olthuis W., Weckhuysen B.M., van den Berg A., MicroTAS 2017 (Poster presentation) Concentration Polarization to Measure Nano-pore Accessibility, September 22-26, 2017, Savannah, United States.

Solsona M., Nieuwelink A.E., Odijk M., Meirer F., Abelmann L., Olthuis W., Weckhuysen B.M., van den Berg A., MicroTAS 2017 (Poster presentation) Magnetophoretic Sorting of Fluid Catalytic Cracking Particles, September 2226, 2017, Savannah, United States.

Solsona M., Nieuwelink A. E., Papadimitriou V., Eijkel J. C. T., Meirer F., Abelmann L., Olthuis W., Weckhuysen B.M., van den Berg A., Eurosensors XXXI, (Poster presentation), Nano-porosity Determined by Ion Concentration Polarization, September 9-12, 2018, Graz, Austria.

Solsona M., Westerbeek E.Y., Olthuis W., van den Berg A. MicroTAS 2018 (Oral presentation) Gradual Capacitance for Particle Tracking in Micro-channels, November 11-15, 2018, Kaohsiung, Taiwan. 


\section{Samenvatting}

Heterogene katalysatoren zijn vaste stoffen die ervoor zorgen dat chemische reacties kunnen verlopen of sneller verlopen. Er zitten echter grote verschillen tussen verschillende katalysatordeeltjes als gevolg van het fabricageproces. Ook binnen een deeltje kunnen vaak meer en minder actieve delen gevonden worden. Over het algemeen gebeurt karakterisering van de activiteit en selectiviteit van deze materialen in batch-reactoren, waardoor slechts gemiddelde resultaten voor de batch bepaald kunnen worden. Dit beperkt de kennis over de individuele rol die elk deeltje speelt en het begrip van de relevante factoren hierin. Daar staat tegenover dat de technieken die nodig zijn om ieder deeltje apart te karakteriseren zeer duur zijn en een lage capaciteit hebben. Er is daarom behoefte aan goedkope systemen waarin individuele deeltjes gekarakteriseerd kunnen worden.

Zoals besproken wordt in hoofdstuk 2, is microfluïdica de afgelopen decennia opgekomen als techniek voor het sorteren en analyseren van losse cellen. Er zijn in de microfluïdische gemeenschap veel technieken ontwikkeld om deeltjes en cellen te manipuleren, sorteren en analyseren. Hierbij wordt gebruik gemaakt van elektriciteit, magnetisme, geluidsgolven of simpelweg de inertie van vloeistoffen.

In dit proefschrift hebben we enkele van deze technieken gebruikt en aangepast en nieuwe technieken ontwikkeld voor het analyseren van individuele katalysatordeeltjes. Op deze manier konden we de brug slaan tussen de karakterisering van individuele katalysatordeeltjes en goedkope technologie en zelfs, waar mogelijk, grote aantallen karakteriseren.

De katalysatordeeltjes die we gebruiken in enkele hoofdstukken zijn zogenaamde fluid catalytic cracking (FCC) deeltjes. Deze poreuze deeltjes met een diameter van 50-150 $\mu \mathrm{m}$ worden in de petrochemische industrie gebruikt voor het kraken van koolwaterstofmoleculen met lange ketens. Hoewel FCC deeltjes al 80 jaar veelvuldig worden toegepast, raken ze inactief door onder andere ijzer (Fe), nikkel (Ni) en vanadium (V) uit de ruwe olie. Om de efficiëntie van de reactor te waarborgen, wordt daarom regelmatig een deel van de FCC deeltjes vervangen door verse deeltjes, wat resulteert in een mengsel van verse en oude deeltjes dat Equilibrium Catalyst (ECAT) wordt genoemd. Nikkel en vanadium verlagen de activiteit van de katalysator doordat ze andere, ongewenste, reacties bevorderen, terwijl ijzer de deeltjes inactief maakt doordat het op de buitenste $2 \mu \mathrm{m}$ neerslaat en vermoedelijk de poriën blokkeert. Met deze kennis in het achterhoofd, ontwikkelden we in hoofdstuk 3 een magnetoforetische, ge3D-printe microfluïdische chip waarmee ECAT FCC deeltjes in 5 verschillende fracties gesorteerd kunnen worden, gebaseerd op hun magnetisch moment. Experimentele resultaten laten zien dat het magnetisch moment gecorreleerd is aan het ijzergehalte van de deeltjes. Daarnaast is uit zogenaamde zero-field cooled 
(ZFC) en field cooled (FC) analyses gebleken dat het ijzer neerslaat in clusters van slechts een paar nanometer groot. Karakterisering van de activiteit heeft laten zien dat deze over het algemeen afneemt met toenemend ijzergehalte, wat bevestigt dat ijzer een van de belangrijke stoffen is die de deeltjes inactief maakt. Opvallend was dat niet alle deeltjes met een hoog ijzergehalte een lage activiteit hadden. Bij nadere visuele inspectie van deze deeltjes viel op dat op deze meer actieve deeltjes zwarte en oranje vlekken te zien waren, die bestonden uit ijzer. Op deeltjes met hoger ijzergehalte waren grotere en meer vlekken zichtbaar. Een mogelijke verklaring voor de hoge activiteit die sommige deeltjes hebben ondanks hun hoge ijzergehalte, is de agglomeratie van ijzer in deze vlekken, waardoor de rest van het deeltje actief blijft.

Om de deeltjes te sorteren op verspreidingsgraad van het ijzer en daarmee het sorteren op activiteit te verbeteren, hebben we een apparaat ontwikkeld waarin de magnetische deeltjes gaan draaien. In het aangebrachte roterende magneetveld ondervinden alleen de deeltjes met inhomogeen verdeeld magnetisch materiaal een moment waardoor ze gaan draaien. Als gevolg hiervan ondervinden deze deeltjes een magnuskracht: een kracht die haaks staat op hun translatierichting. Vanwege het ontbreken van literatuur over dit fenomeen op de microschaal, richt hoofdstuk 4 zich op het aantonen van mogelijkheid om de magnuskracht te gebruiken voor het sorteren van microdeeltjes. De experimentele resultaten tonen aan dat de magnuskracht de deeltjes die door een kanaal stromen kan laten uitwijken en daarom gebruikt kan worden voor het sorteren van microdeeltjes of cellen.

Wanneer FCC deeltjes inactief worden door accumulatie van metaal, veranderen ook de diëlektrische eigenschappen. Voor de meeste neergeslagen metaaloxides geldt dat hun diëlektrische constante en geleidbaarheid groter zijn dan die van de belangrijkste bestanddelen van de deeltjes: silica en alumina. In de biologie wordt flowcytometrie gebruikt om de individuele diëlektrische eigenschappen van grote aantallen cellen te bepalen. In hoofdstuk $\mathbf{5}$ ontwikkelden we daarom een microfluïdische chip om de diëlektrische eigenschappen van individuele FCC deeltjes te bepalen. Hoewel we het systeem experimenteel gevalideerd hebben, hebben we geen verschil in diëlektrische eigenschappen tussen FCC deeltjes met verschillend metaalgehalte kunnen meten.

Bij impedantie-flowcytometrie is de positie tussen de elektrodes van de cel of het deeltje van groot belang, als gevolg van het zogenaamde fringing effect waardoor het elektrisch veld niet perfect homogeen is. Hierdoor leveren twee identieke deeltjes verschillende signalen wanneer ze op twee verschillende posities tussen de elektrodes door stromen. Dit kan opgelost worden door het detecteren van de positie en het corrigeren van het gemeten signaal. Hoofdstuk 6 behandelt een nieuwe methode om de positie van microdeeltjes tussen twee elektrodes te bepalen. Hierbij wordt gebruikgemaakt van ongelijkmatig aangebracht platinazwart op twee parallel uitgelijnde elektrodes. Op deze 
manier kan de positie van de deeltjes worden gevolgd bij lage frequenties ( $\mathrm{Hz}$ ), terwijl tegelijkertijd de geleidbaarheid wordt gemeten bij hoge frequenties (100 $\mathrm{kHz}$ ).

Zoals eerder genoemd, accumuleert het ijzer in de buitenste $2 \mu \mathrm{m}$ van de FCC deeltjes en blokkeert het daarbij vermoedelijk de buitenste poriën, waardoor reagentia de deeltjes niet meer in kunnen en deze inactief raken. Porositeitsbepalingen worden over het algemeen óf uitgevoerd in grote vaten, óf er wordt gemeten aan individuele deeltjes. In het eerste geval worden gemiddelde waarden verkregen voor meerdere deeltjes, in het tweede geval deeltjes wordt er dure technologie gebruikt waarmee geen grote aantallen individuele deeltjes bemonsterd kunnen worden. Er zijn daarom nieuwe methoden nodig waarmee de porositeit van individuele deeltjes bepaald kan worden. Ion concentratie polarisatie (ICP) treed op aan het interface tussen een ion-permselectief materiaal en een oplossing wanneer een elektrisch veld wordt aangelegd. Hierdoor ontstaat als gevolg van onbalans in de ionenflux respectievelijk een accumulatiezone en een depletiezone aan weerszijden van het materiaal. Poreuze materialen worden ion-permselectief wanneer afmeting van de poriën in dezelfde ordegrootte is als de dikte van de elektrische dubbellaag aan de wanden van de poriën (meestal nanometerschaal). In hoofdstuk 7 deden we twee typen polystyrenen deeltjes met nanoporiën van gemiddeld $3 \mathrm{~nm}$ en $13 \mathrm{~nm}$ in een eenvoudige microfluïdische chip van PDMS en gebruikten ICP om het verschil tussen beide te zien. Daarnaast ontwikkelden we een kwalitatief model dat de concentratie van een fluorescente marker in de accumulatie- en depletiezones kan voorspellen. Dit model is experimenteel gevalideerd.

Karakterisering van de activiteit en selectiviteit van een katalysator wordt normaliter gedaan in reactoren met duizenden tot miljarden deeltjes, waardoor het resultaat een gemiddelde is. Zover wij weten is de activiteit en selectiviteit van individuele katalysatordeeltjes nooit bepaald. In hoofdstuk 8 ontwikkelden we een microreactor voor individuele katalysatordeeltjes, waarin individuele ECAT FCC deeltjes werden gebracht. N-hexaan werd gebruikt als reagens en tijdens het katalytische proces werd een hogere fluorescentie gemeten, mogelijk vanwege hoger koolstofgehalte in de deeltjes als gevolg van het kraken van nhexaan. Daarnaast nam de fluorescentie af na introductie van zuurstof in de reactoren, mogelijk als gevolg van koolstofverbranding en -verwijdering. 


\section{Contributions}

\section{Chapter 2}

The work of this chapter was divided in 3 sections: synthesis, characterization and future perspectives. Jeroen Vollenbroek and Coretin B.M. Tregouet were responsible for the characterization and the future perspective sections respectively.

\section{Chapter 3}

This chapter resulted from a collaboration between the Inorganic Chemistry and Catalysis group in Utrecht University and BIOS Lab on a chip group in University of Twente. Anne-Eva Nieuwelink carried out the XRF, acidity, EPR-UV-vis and surface area of black spots analysis on sorted FCC particles. Hans de Boer fabricated the microfluidic chip holder.

\section{Chapter 4}

This chapter resulted from the previous work done by Hans Keizer. The rotating magnetic field setup design was performed by Leon Abelmann and Hans de Boer. The construction of the setup was performed by the workshop at University of Twente.

\section{Chapter 5}

Jordi Hendrix carried out the experiments with PS particles in anisole. The fabrication of the microfluidic chip was performed in collaboration with Jordi Hendrix and Johan Bomer. Hans de Boer fabricated the faraday cage.

\section{Chapter 6}

The experiments were performed by Eiko Westerbeek. The microfluidic chip was fabricated in collaboration with Eiko Westerbeek and Johan Bomer.

\section{Chapter 7}

This chapter was divided in 2 sections: simulation and experimental proof. Vasileios Papadimitriou was responsible for the simulation section.

\section{Chapter 8}

This chapter resulted from the collaboration with Anne-Eva Nieuwelink and Jeroen Vollenbroek. 


\section{Acknowledgments}

Well, this is it. This is the moment to thank everybody that helped me throughout these 4 years.

I would like to start with my daily supervisor, Dr. Wouter Olthuis. It has been a pleasure working with you. I will always remember the sentence "you should convince me first" that you always told me when I wanted to do a new experiment. Also, I would like to thank you for trusting me when I went to Barcelona so often to "work" from home. You have been a very nice and helpful supervisor.

Also, I would like to thank my promotor Prof. dr. ir. Albert van den Berg. You always gave me the right comments on the papers and presentations, pointing at things that I didn't think about. You also helped me whenever I asked you for any favour and I am very grateful for that. I also want to show my sincere gratitude to Dr. Mathieu Odijk for helping me out every time he could. You were always willing to discuss new ideas or experiments. Even when I asked you if you had " 1 minute" and it turned out being a 15 minutes discussion. Also, I want to thank Dr. Loes Segerink. You really helped me to understand better all those projects related to impedance!

I also want to show my gratitude to Prof. dr. J.C.T. Eijkel. We had a lot of discussions and meetings, and I have learned a lot from you. I sometimes needed a couple of hours to understand what you were trying to explain during the meetings. It has been a pleasure to work with you!

I also want to thank Prof. Bert M. Weckhuysen for your motivation and patience during our collaborations. You definitely taught me a lot about the scientific world! And of course, I want to thank Prof. Leon Abelmann. I actually think that my PhD changed when I met you!

Beyond the staff members, there is another group of people that I want to give a huge thanks: the technicians of the group. Paul, I will always remember your jokes even when you repeat them during my whole PhD. Besides that, thanks for being very helpful and ready to help. I want to thank Johan for all the time he spent with me in the cleanroom. I really think that without your help I could have never achieved what I did during my PhD. I also want to thank Hans for helping me building almost all the setups that I used. And thanks to Jan for all the interesting conversations about the setups in our group. You really helped me in the beginning of my PhD.

Beyond the staff members and technicians, there is a large number of people that made my stay in Twente much better. I want to start with my first officemates, Renée and Hai. You guys made the beginning of my PhD much easier. Hai, I want to thank you for the endless laugh and happiness. Renée, many thanks for being so positive and willing to help me with the Dutch letters 
that I didn't understand. And now my current officemates, Juan and Vasili. It has been a pleasure to share the office with you guys. We got a lot of fun!

I also want to show my gratitude to the students that helped me to complete my thesis, Hans, Jordi, Eiko and Martijn. I am very grateful for all your great work!

And now the rest of the group. I am sure I will forget someone but here it goes. Thanks to: Hugo, Anke, Ruben, Pelin, Martijn, Laura, Lisette, Tanja, Alessia, Cristina, Josh, Jorien, Maria, Rob, Jeroen, Stefan, Luca, Lanhui, Floris, Elsbeth, Jasper, Esther, Pascal, Alessandro, Mariia, Douwe, Nienke, Marinke, Miguel, Bjorn, Rogier, Burcu, Adithia, Anne, Julia, Dodo, Edo, Thieu, Jawar, Wesley, Susan, Allison, Aura, Jean-Baptiste and Bastien. You guys made all these years much more joyful.

And of course, I didn't forget the MCEC community. It was very fun to share endless meetings with you guys. More specifically, I would like to give my gratitude to Anne-Eva and Florian for the nice collaborations!

Beyond the scientific world, I also met a lot of people that helped me during my stressful moments. I want to thank the Drienerlo football team for the fun and sporty moments that you guys shared with me. Also, I want to thank the Cabezota handball team for being so nice and welcoming. I really enjoyed the trainings and matches that we played together.

$\mathrm{Y}$ ara vull donar-te les gràcies a tu Anna. Gràcies per haber-me ajudat tant durant aquests 4 anys. Gràcies per haber vingut a Holanda tantes i tantes vegades. I sobretot gràcies per haber-me donat tanta força. També vull agraïr el suport a tots els meus amics. Sense vosaltres tot hagués sigut més difícil. Y como no, mi familia. Gracias a mis padres por estar siempre ahí. Habéis sido y sois los mejores padres que podría tener. Siempre me habéis apoyado en todo lo que he querido hacer. No se cuantas veces me habréis recogido y dejado en el aeropuerto durante estos años. Os quiero y siempre os querré. Gracias por ser como sois. Y como no mi hermanito. Aunque se que no eres la persona más expresiva de la historia, quiero darte las gracias por ser tan buena persona y siempre ayudarme cuando te lo he pedido. Gracias hermanito. También quiero dar las gracias a mi yaya, mi segunda madre. Aunque ya no puedas estar con nosostros se que estarías orgullosa de mi. Gracias por querernos tanto. Te quiero.

Miguel Solsona

Enschede, April 2019 
